Supporting Information

\title{
Mutations in Dynamic Structural Elements Alter the Kinetics and Fidelity of the Multifunctional Class II Lanthipeptide Synthetase, HalM2
}

\author{
Kevin A. Uggowitzer, ${ }^{1}$ Yeganeh Habibi, ${ }^{1}$ Wanlei Wei, ${ }^{1}$ Nicolas Moitessier, ${ }^{1}$ and \\ Christopher J. Thibodeaux ${ }^{1,2, *}$ \\ ${ }^{1}$ Department of Chemistry \\ ${ }^{2}$ Centre de Recherche en Biologie Structurale \\ McGill University \\ 801 Sherbrooke St West \\ Montréal, Québec, Canada, H3A 0B8
}

*to whom correspondence should be addressed

christopher.thibodeaux@mcgill.ca

1-(514)-398-3637 


\section{Table of Contents}

\section{Supporting Methods:}

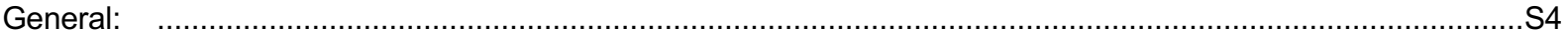

Oligonucleotide Sequences:

Cloning strategy and PCR amplification of fragments for Gibson assembly ...................................................

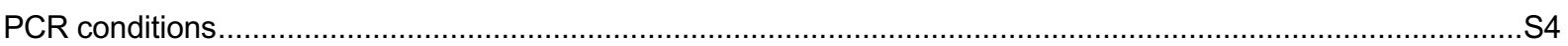

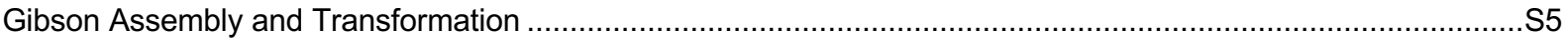

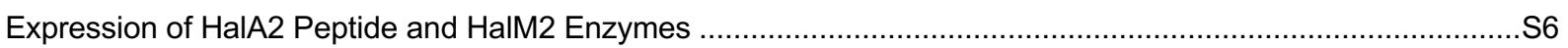

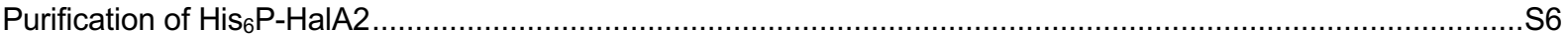

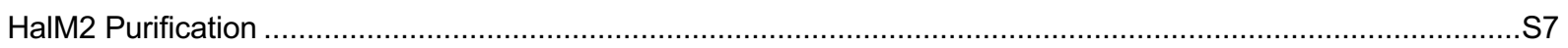

Fluorescence Polarization Experiments ………………

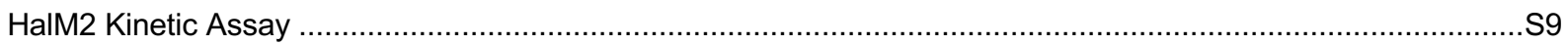

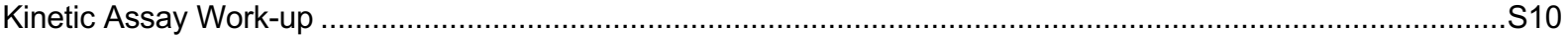

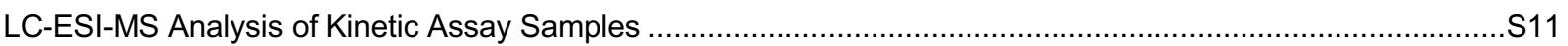

Determining the relative abundance of HalM2/HalA2 reaction intermediates ...................................................S11

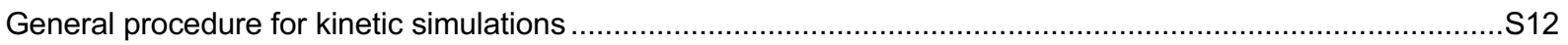

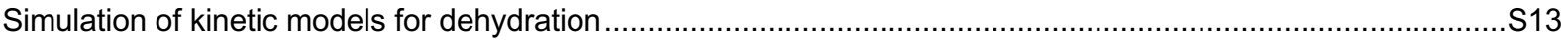

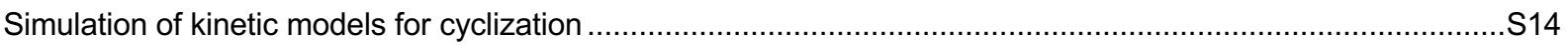

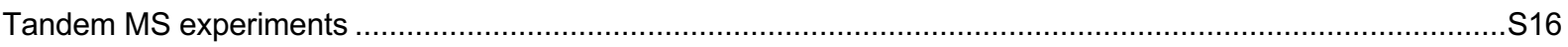

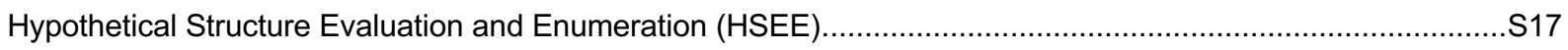

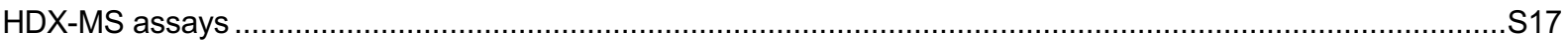

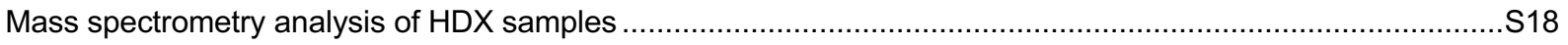

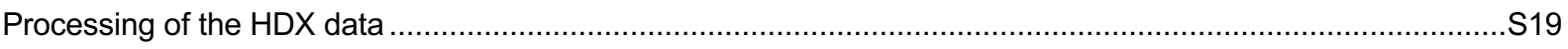

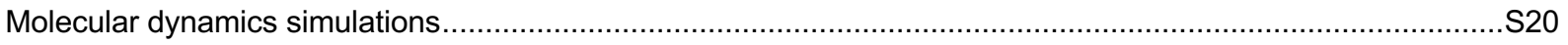

\section{Supporting results:}

Figure S1. Fluorescence polarization experiments of HalM2 variants ......................................................... 22

Figure S2. Comparison of new vs. previously reported simulation of wt HalM2 reaction..................................S23

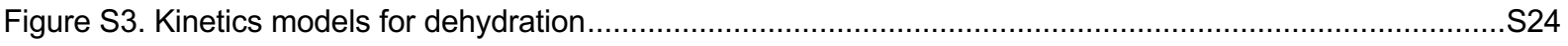

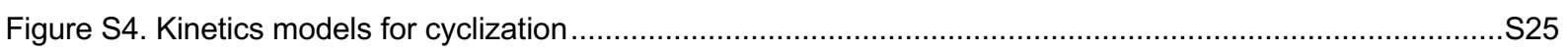

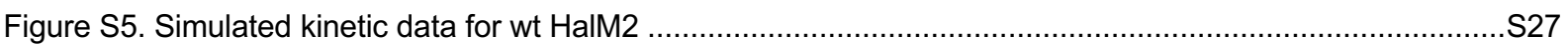

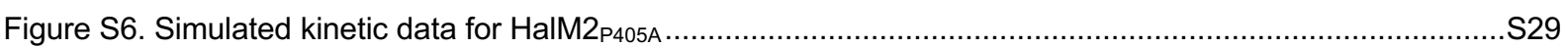

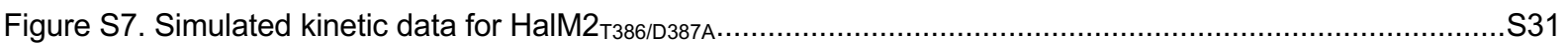

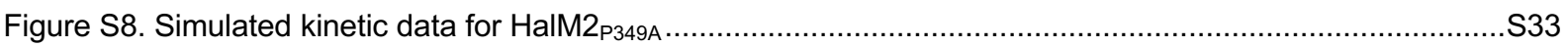

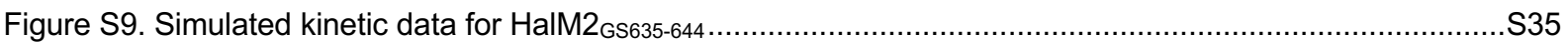

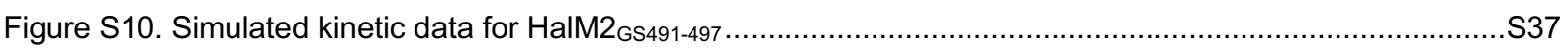

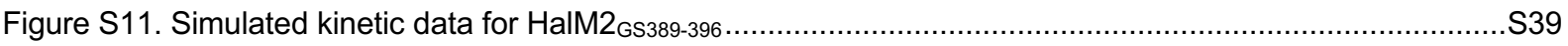

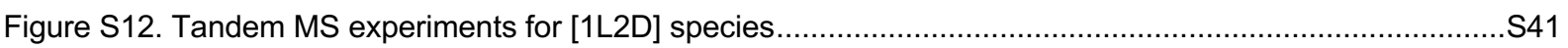

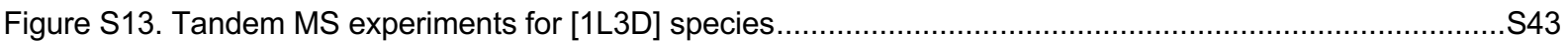

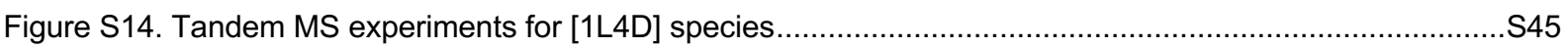

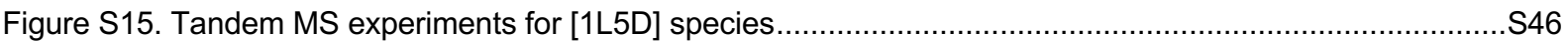

Figure S16. Tandem MS experiments for [2L2D] species..................................................................... 47 
Figure S17. Tandem MS experiments for [2L3D] species. S49

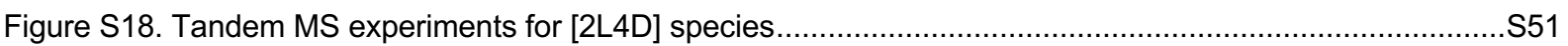

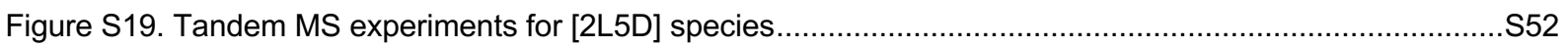

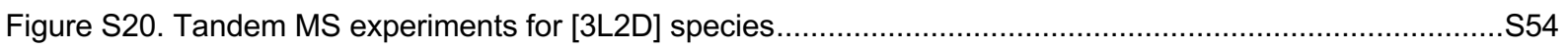

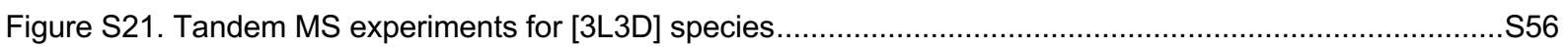

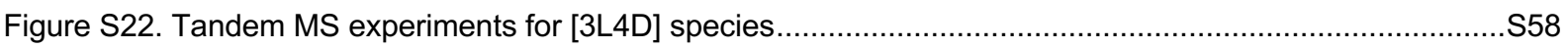

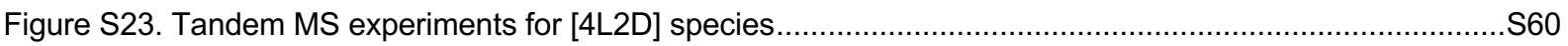

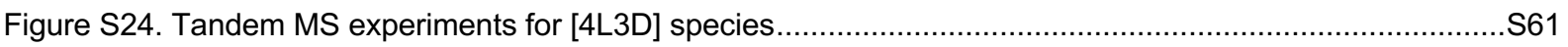

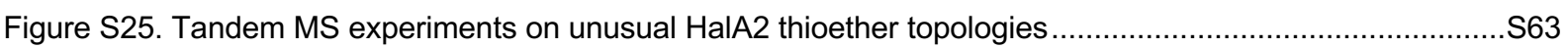

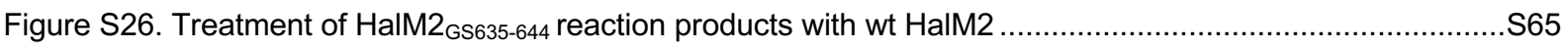

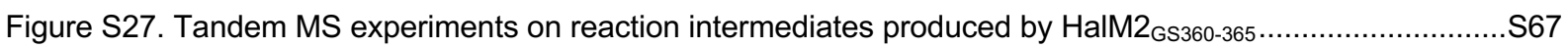

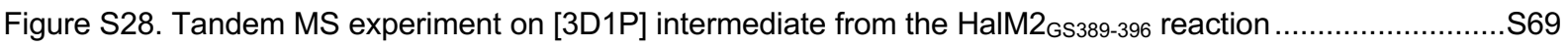

Figure S29. Tandem MS experiment on [1L2D1P] intermediate from the HalM2 ${ }_{\mathrm{GS} 389-396}$ reaction .......................S70

Figure S30. Tandem MS experiment on [3L2D1P] intermediate from the HalM2GS389-396 reaction .....................S71

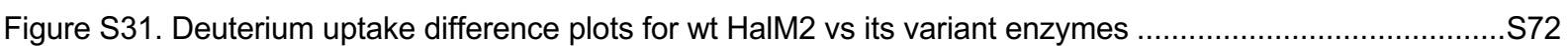

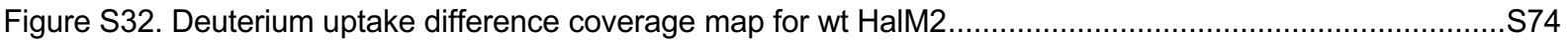

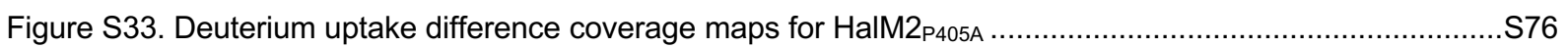

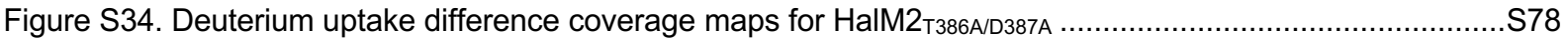

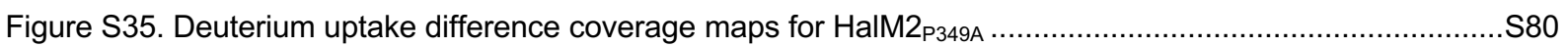

Figure S36. Deuterium uptake difference coverage maps for HalM2GS635-644 ..............................................

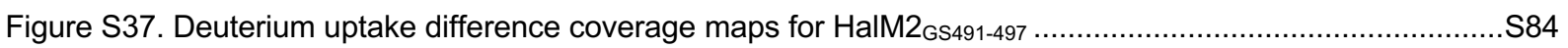

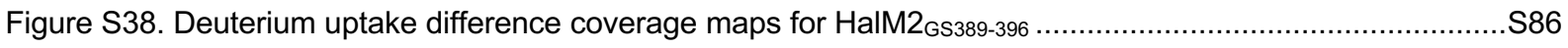

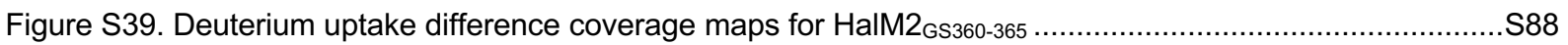

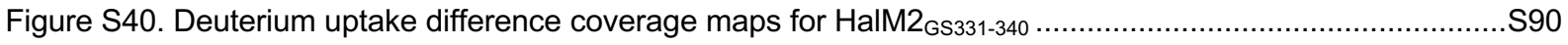

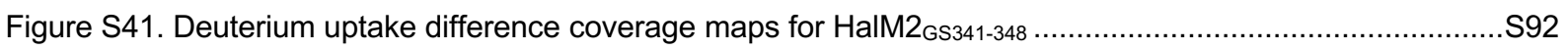

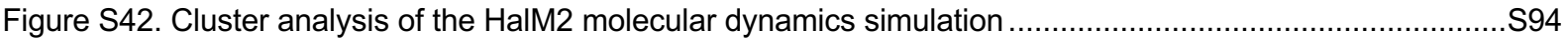

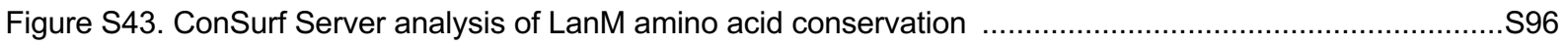

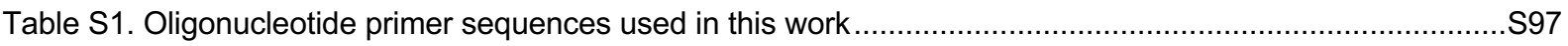

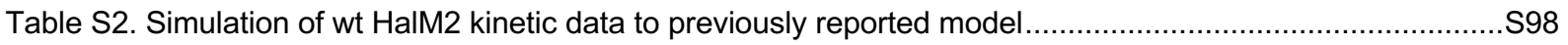

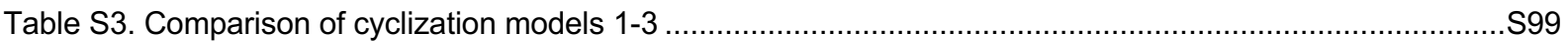

Table S4. Simulated kinetic parameters for the HalM2 ${ }_{\text {GS389-396 }}$ enzyme ................................................ 100

Table S5. Variant peptides that undergo significant deuterium uptake changes relative to free wt HalM2 ........S101

Table S6. Variant peptides that undergo significant deuterium uptake changes in the presence of ligands ......S108

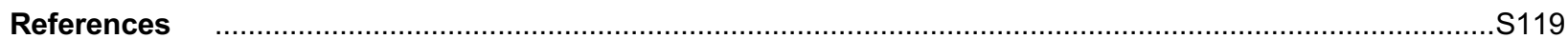




\section{Supporting Methods}

General: All DNA sequencing was performed by the Génome Québec Innovation Centre at McGill University. MALDI-TOF MS was performed on a Bruker Autoflex III in the mass spectrometry facility at McGill University. All restriction enzymes and other molecular genetics reagents were purchased from New England Biolabs unless otherwise noted. The NCBI accession numbers of LanM enzymes and LanA peptides of relevance to this study: CyIM (AAK67266.1), HalM2 (WP_010896630.1), HalA2 (WP_010896631.1), ProcM (WP_011129629.1), ProcA2.8 (WP_011131486.1).

Oligonucleotide Sequences: The sequences of all oligonucleotide primers used in this study are displayed in Table S1. All oligonucleotide primers were ordered from Integrated DNA Technologies (IDT). All primers were prepared as $10 \mu \mathrm{M}$ stock solutions in sterile $\mathrm{H}_{2} \mathrm{O}$ and used without further purification. Additional information regarding the design of individual primers is provided as necessary below during the description of the specific Gibson assemblies for which they were used.

Cloning strategy and PCR amplification of fragments for Gibson assembly: To mutate the wt halm2 gene using the Gibson assembly, we designed sets of primers to amplify two fragments from the corresponding halM2/pET15b template. The primers were designed such that, following PCR amplification, the two fragments would have $\sim 30 \mathrm{bp}$ overlaps in the region of the halM2 gene containing the desired mutation and in the region of the vector encoding for the antibiotic resistance gene. With this arrangement, the desired mutations could be introduced using the PCR primers and only successful assemblies should result in plasmid DNA products that confer antibiotic resistance. The primer sets used to amplify the Gibson fragments for the HalM2P405A, HalM2 T386A/D387A, HalM2P349A, and HalM2GS360-365 variants are listed in Table S1. The construction of the other HalM2 variants used in this study were described previously. ${ }^{1}$

PCR conditions: All PCR reactions (50 $\mu \mathrm{L})$ contained $1 \mathrm{X}$ Phusion HF buffer, $5 \%$ DMSO, $0.5 \mu \mathrm{M}$ of each primer, $50-250 \mathrm{ng}$ of template DNA, $200 \mu \mathrm{M}$ dNTPs and $1 \mathrm{U}$ Phusion DNA 
Polymerase. All reagents (excluding the primers and template DNA) were purchased from New England biolabs (NEB). If the desired Gibson fragment was $>3 \mathrm{kbp}, 3 \times 50 \mu \mathrm{L}$ PCR reactions were run simultaneously to increase the final PCR product yield after purification. High fidelity Phusion DNA polymerase was used to minimize unwanted mutations during amplification. The following cycling conditions were used unless otherwise noted. Initial denaturation of DNA template was carried out at $98{ }^{\circ} \mathrm{C}$ for $30 \mathrm{~s}$. Next, amplification was achieved in 35 cycles of: denaturation at $98^{\circ} \mathrm{C}$ for $10 \mathrm{~s}$, annealing at $55-65{ }^{\circ} \mathrm{C}$ (depending on the primer pair) for $30 \mathrm{~s}$, and extension at $72{ }^{\circ} \mathrm{C}$ for $30 \mathrm{~s}$ per $\mathrm{kbp}$ of the fragment to be amplified. The last step is a final extension at $72{ }^{\circ} \mathrm{C}$ for $10 \mathrm{~min}$ followed by hold at $4{ }^{\circ} \mathrm{C}$. PCR products were incubated with Dpn1 restriction endonuclease (NEB, item R0176) at $37^{\circ} \mathrm{C}$ for $1 \mathrm{~h}$ to remove the template plasmid and were then purified using $0.8 \%$ agarose gel electrophoresis. The gel and running buffer were prepared in $40 \mathrm{mM}$ tris-acetate, $20 \mathrm{mM}$ acetic acid and 1mM EDTA (Tris-acetateEDTA) TAE buffer. Following electrophoresis, bands were extracted from the gel and the Gibson fragments were further purified using the Takara PCR cleanup and gel extraction kit (Cat\#: 740609). After purification, the concentration of each fragment was determined by UV absorption using a Thermo Scientific Nanodrop Lite, and the fragments were subsequently stored at $-20^{\circ} \mathrm{C}$ until use.

Gibson Assembly and Transformation: To prepare a typical assembly, a $5 \mu \mathrm{L}$ solution containing $100 \mathrm{ng}$ of the larger DNA fragment and an equimolar amount of the smaller fragment was made. A $15 \mu \mathrm{L}$ aliquot of Gibson mix was defrosted on ice and added to the Gibson fragment mixture. The Gibson mix contained 5\% PEG-8000, 100 mM Tris$\mathrm{HCl} \mathrm{pH} \mathrm{7.5,} 10 \mathrm{mM} \mathrm{MgCl}$, $10 \mathrm{mM}$ DTT, $200 \mu \mathrm{M}$ dNTPs, 1 mM NAD $80 \mathrm{U}$ of taq DNA ligase (NEB, item M0208), $0.5 \mathrm{U}$ of Phusion DNA polymerase (NEB, item M0531), and $0.08 \mathrm{U}$ of T5 exonuclease (NEB, item M0363). The sample was immediately incubated in a water bath at $50^{\circ} \mathrm{C}$ for 1 hour. After incubation, $10 \mu \mathrm{L}$ of this mixture was transformed directly into $50 \mu \mathrm{L}$ of chemically competent $E$. coli DH5a. After addition, the cells were incubated on ice for $30 \mathrm{~min}$. After incubation, the cells were heat shocked in a water bath at $42{ }^{\circ} \mathrm{C}$ for $30 \mathrm{~s}$, and immediately put back on ice for $2 \mathrm{~min}$. Using aseptic techniques, 1 $\mathrm{mL}$ of Luria broth (LB) media was as added to the cells and incubated in $37{ }^{\circ} \mathrm{C}$ for $1 \mathrm{~h}$ in 
shaker at $160 \mathrm{rpm}$. After incubation, $100 \mu \mathrm{L}$ of this mixture was applied to LB agar plates containing the necessary antibiotic $(100 \mu \mathrm{g} / \mathrm{mL}$ ampicillin when using $p E T 15 b$, or 30 $\mu \mathrm{g} / \mathrm{mL}$ kanamycin when using $p E T 28 b$ or $p R S F-D U E T)$. After allowing the plate to dry for $10 \mathrm{~min}$, the plate was placed inverted in the incubator at $37^{\circ} \mathrm{C}$ for $18 \mathrm{~h}$.

Expression of HalA2 Peptide and HalM2 Enzymes: First, 6 L of Luria broth (LB) media was prepared and autoclaved. Next, a $60 \mathrm{~mL}$ overnight culture of $E$. coli BL21 transformed with the plasmid of interest and the appropriate antibiotic was prepared and allowed to grow for $18 \mathrm{~h}$ at $37^{\circ} \mathrm{C}$ with shaking at $170 \mathrm{rpm}$. The $6 \mathrm{~L}$ of LB media was inoculated with $10 \mathrm{~mL}$ of overnight culture per $\mathrm{L}$ along with appropriate antibiotic. The shaking speed was set to $170 \mathrm{rpm}$ and the culture was incubated at $37^{\circ} \mathrm{C}$ for two hours. After two hours, the OD600 was monitored using a UV-Vis spectrophotometer until the cultures reached a reading of 0.8 . After reaching this stage, the cultures were induced with isopropyl $\beta$-D-1thiogalactopyranoside (IPTG) to give a final concentration of $1 \mathrm{mM}$. After induction, cultures for expressing HalA2 peptide were grown for 3 additional $\mathrm{h}$ at $37^{\circ} \mathrm{C}$. The cells were then pelleted at $6000 \times \mathrm{g}$ for $20 \mathrm{~min}$ and stored at $-80{ }^{\circ} \mathrm{C}$ until ready for purification. Expression of HalM2 enzymes was conducted in an identical manner except that protein expression was induced with $250 \mu \mathrm{M}$ IPTG when the OD600 reached 0.7. The cultures were then incubated at $18^{\circ} \mathrm{C}$ overnight and pelleted at $6000 \mathrm{xg}$ for $20 \mathrm{~min}$. After pelleting, the cells were stored at $-80^{\circ} \mathrm{C}$ until ready for purification.

Purification of His ${ }_{6}$ P-HalA2: Frozen cell pellets were defrosted and re-suspended with LanA wash buffer $\left(20 \mathrm{mM} \mathrm{NaH}_{2} \mathrm{PO}_{4}, 500 \mathrm{mM} \mathrm{NaCl}, 0.5 \mathrm{mM}\right.$ imidazole, $6 \mathrm{M}$ guanidinium$\mathrm{HCl}, \mathrm{pH} \mathrm{7.5)}$ in a metal beaker. The pellet was sonicated at room temperature for $15 \mathrm{~min}$ with $4.0 \mathrm{~s}$ burst followed by $9.9 \mathrm{~s}$ rest period. After sonication, the lysate was centrifuged at $15,000 \times g$ for $45 \mathrm{~min}$ to pellet cellular debris at $4{ }^{\circ} \mathrm{C}$. The subsequent purification process was conducted at room temperature. A $5 \mathrm{~mL}$ HisTrap FF Ni-NTA column (GE Healthcare) was pre-equilibrated in LanA wash buffer with a peristaltic pump at 1.5 $\mathrm{mL} / \mathrm{min}$. The supernatant from the centrifuged cell lysate was loaded on to the Ni-NTA column with a $1.5 \mathrm{~mL} / \mathrm{min}$ flow rate. After loading, the column was washed with 10 column volumes $(\mathrm{CV})$ of LanA wash buffer. After washing, LanA was eluted off the column with 5 
CV of LanA elution buffer $\left(20 \mathrm{mM} \mathrm{NaH} \mathrm{PO}_{4}, 100 \mathrm{mM} \mathrm{NaCl}, 1 \mathrm{M}\right.$ imidazole, $4 \mathrm{M}$ guanidinium- $\mathrm{HCl}, \mathrm{pH}$ 7.5). The Ni-NTA eluent was desalted with a Waters C8 Sep-Pak column (WAT054660). The column was pre-equilibrated with $10 \mathrm{CV}$ of $0.1 \%$ trifluoroacetic acid (TFA) in water. After pre-equilibration the $\mathrm{His}_{6} \mathrm{P}-\mathrm{Hal} \mathrm{A} 2$ containing sample was loaded onto column. When loaded, the column was washed with $10 \mathrm{CV}$ of $0.1 \%$ TFA solution to remove salts. His 6 P-HalA2 was then eluted with $5 \mathrm{CV}$ of $80 \%$ acetonitrile (ACN) with $0.1 \%$ TFA in water. The sample was lyophilized (at $-86{ }^{\circ} \mathrm{C}$ ) until dry and stored at $-80{ }^{\circ} \mathrm{C}$ until further purification.

The lyophilized sample was resuspended in $15 \mathrm{~mL}$ of $0.1 \%$ TFA and centrifuged at 16,100 $x g$ for 1 min to pellet any precipitant. The supernatant was collected and injected in $6 \mathrm{~mL}$ portions onto a $\mathrm{C}_{8}$ Supelco Reverse Phase Semi-Prep Column (Sigma Aldrich, $25 \mathrm{~cm} \mathrm{x}$ $10 \mathrm{~mm}, 10 \mu \mathrm{m} 567222-\mathrm{U}$ ) for HPLC purification. Solvent A was $0.1 \%$ TFA and Solvent B was ACN with $0.1 \%$ TFA. The column was eluted with a gradient of $3-100 \%$ B over 37 min. Hal6P-HalA2 eluted at $28 \mathrm{~min}$. Fractions were pooled and checked for His6P-HalA2 using MALDI-TOF MS. Fractions containing product were pooled and lyophilized to dryness. After lyophilization, $\mathrm{His}_{6} \mathrm{P}-\mathrm{Hal} \mathrm{A} 2$ was re-suspended in water to produce aliquots which were stored short-term at $-20{ }^{\circ} \mathrm{C}$ and $-80{ }^{\circ} \mathrm{C}$ for long-term storage.

HalM2 Purification: All HalM2 enzymes in this work were purified by the following procedure. First the pellet was resuspended in LanM lysis buffer $(500 \mathrm{mM} \mathrm{NaCl}, 20 \mathrm{mM}$ Tris, $10 \%$ glycerol, $\mathrm{pH} 7.5$ ) in a metal beaker on ice. The pellet was then sonicated (using $60 \%$ amplitude) using $2.0 \mathrm{~s}$ pulses separated by $8.0 \mathrm{~s}$ off for $2-5$ min depending on the size of pellet. After sonication, the cell debris was centrifuged at $15,000 \times g$ for $45 \mathrm{~min}$ at $4{ }^{\circ} \mathrm{C}$. A $5 \mathrm{~mL}$ HisTrap FF column (GE Healthcare) was pre-equilibrated with $5 \mathrm{CV}$ of LanM lysis buffer at a flow rate of $1.5 \mathrm{~mL} / \mathrm{min}$ using a peristatic pump. After centrifugation, the supernatant was carefully collected, separated from the pellet and kept on ice until the column had been pre-equilibrated. After column pre-equilibration, the supernatant was loaded onto the column at a flow rate of $1.5 \mathrm{~mL} / \mathrm{min}$ with the peristaltic pump at $4{ }^{\circ} \mathrm{C}$. After the supernatant was loaded onto the column, the column was washed with $5 \mathrm{CV}$ of LanM wash buffer ( $1 \mathrm{M} \mathrm{NaCl}, 20 \mathrm{mM}$ Tris, $30 \mathrm{mM}$ imidazole, $\mathrm{pH}$ 8.0). After washing, the column 
was placed on a BioRad NGC Quest 10 Fast Protein Liquid Chromatography (FPLC) system. The column was washed with LanM wash buffer until the $A_{280}$ reading reached the baseline, indicating elution of all the non-bound protein material. The column was then eluted with a linear gradient of $0-100 \%$ B $(500 \mathrm{mM} \mathrm{NaCl} 20 \mathrm{mM}$ Tris $200 \mathrm{mM}$ imidazole, $\mathrm{pH} 7.5)$ at $1.5 \mathrm{~mL} / \mathrm{min}, 4^{\circ} \mathrm{C}$. Fractions $(5 \mathrm{~mL})$ containing protein were pooled, spiked with glycerol to $10 \%$ and concentrated to about $3 \mathrm{~mL}$ using an Amicon Ultra-15 Centrifugal filter with a $50 \mathrm{kDa}$ MW cutoff. For concentration, samples were centrifuged at $5000 \mathrm{xg}$ for 10 min at $4{ }^{\circ} \mathrm{C}$. After concentration, to remove any precipitant the sample was centrifuged at $16,100 \times g$ for $1 \mathrm{~min}$ before proceeding. The supernatant was further purified by size exclusion chromatography using an HiLoad 16/600 superdex 200 prep grade Size Exclusion Chromatography (SEC) column $(1 \times 120 \mathrm{~mL}$, GE Healthcare 28989335). The column was pre-equilibrated with gel filtration buffer (20 mM HEPES, pH $7.5,300 \mathrm{mM} \mathrm{KCl}$ and $10 \%$ glycerol) at $1.0 \mathrm{~mL} / \mathrm{min}$ before injection. After equilibration, 3 $\mathrm{mL}$ of the concentrated Ni-NTA eluent was injected into the system and purified using an isocratic elution in gel filtration buffer. LanM enzymes typically elute in different oligomeric states, with aggregate eluting first followed by oligomer and monomer. Fractions containing oligomer and monomer were pooled and their concentration was determined by measuring the absorption at $280 \mathrm{~nm}$ and by using the calculated extinction coefficients for HalM2 $\left(\varepsilon_{280}=113,020 \mathrm{M}^{-1} \mathrm{~cm}^{-1}\right)$. The proteins were then aliquoted, flash frozen in liquid nitrogen and stored at $-80^{\circ} \mathrm{C}$.

Fluorescence Polarization Experiments: The HalA2 leader peptide was synthesized by BioBasic with a 5 -carboxyfluorescein (5-FAM) tag on the $\mathrm{N}$-terminus and was stored as a $10 \mu \mathrm{M}$ stock solution in water at $-80^{\circ} \mathrm{C}$. This peptide will be subsequently referred to as fluoro-HalA2-LP. To establish the linear range for the fluoro-HalA2-LP fluorescence emission, serial dilutions spanning a concentration range of $1 \mu \mathrm{M}-7.8 \mathrm{nM}$ were prepared in 1x LanM kinetic assay buffer (100 mM HEPES pH 7.5, $5 \mathrm{mM} \mathrm{MgCl2,} 0.5 \mathrm{mM}$ TCEP). Samples were prepared in a Corning solid black 96 -well plate and the fluorescent signal of these samples was measured $\left(\lambda_{e x}=492 \mathrm{~nm}, \lambda_{e m}=518 \mathrm{~nm}\right.$,) using a SpectraMax M5e multi-detection reader. These values were plotted against fluoro-HalA2-LP concentration and fit with a line to determine the figures of merit. From this analysis, we chose $250 \mathrm{nM}$ 
fluoro-HalA2-LP as the optimum concentration for subsequent assays. To conduct the titration, a $250 \mu \mathrm{L}$ solution of $100 \mu \mathrm{M}$ HalM2 was prepared in LanM kinetic assay buffer in a Corning solid black 96-well plate. This solution was mixed 1:1 with $500 \mathrm{nM}$ fluoroHalA2-LP (also prepared in LanM kinetic assay buffer) to give $200 \mu \mathrm{L}$ of a solution containing $50 \mu \mathrm{M}$ HalM2 and $250 \mathrm{nM}$ fluoro-HalA2 leader in LanM kinetic assay buffer. Serial dilutions were then prepared by sequential $1: 1$ mixing with $100 \mu \mathrm{L}$ portions of 250 nM fluoro-HalA2-LP in assay buffer, such that the HalM2 concentration was reduced by half with each subsequent mix, while the fluoro-HalA2-LP concentration remained constant. The serial dilution series was prepared in duplicate from two separate initial mixtures. The fluorescence polarization (FP) measurements were then carried out on a SpectraMax M5e multi-detection reader as described above. The raw values for fluorescence intensity measured in the perpendicular $(S)$ and parallel $(P)$ directions were corrected to account for scattering (as measured in a control dilution series lacking fluoroHalA2-LP) prior to calculation of the FP value via equation 1. The data were subsequently fitted to equation 1 to estimate the HalM2: fluoro-HalA2-LP binding affinity.

$$
\text { Fluorescence Polarization }(m P)=\frac{P-S}{P+S} \times 1000
$$

Equation 1: Used to calculate Fluorescence polarization from raw data with fluorescence parallel $(P)$ and fluorescence perpendicular $(S)$.

$$
P=\text { constant }+\frac{P \max }{K d+[\text { HalM2 }]}
$$

Equation 2: Used to estimate the $K_{d}$ of HalM2: fluoro-HalA2-LP binding affinity where $\mathrm{P}$ is fluorescence polarization.

HalM2 Kinetic Assay: Reactions were conducted as described previously. ${ }^{2}$ Aliquots of HalM2 and His6P-HalA2 were defrosted on ice. After defrosting, the concentration of each stock solution was determined in triplicate by measuring the absorption at $280 \mathrm{~nm}$ (HalM2 $\varepsilon_{280}=113,020 \mathrm{M}^{-1} \mathrm{~cm}^{-1}$, His6P-HalA2 $\varepsilon_{280}=5,514 \mathrm{M}^{-1} \mathrm{~cm}^{-1}$ ). Fresh aliquots of $100 \mathrm{mM}$ TCEP and $100 \mathrm{mM}$ ATP were prepared and kept on ice. Stocks of $1 \mathrm{M}$ HEPES pH 7.5 
and $500 \mathrm{mM} \mathrm{MgCl} 2$ were prepared and stored on the benchtop. Two separate solutions were prepared in order to allow for the HalM2 enzyme and His6P-HalA2 peptide substrate to equilibrate in the buffer conditions prior to $1: 1(\mathrm{v}: \mathrm{v})$ mixing to initiate the reaction. The peptide solution contained $100 \mu \mathrm{M}$ His6P-HalA2, $100 \mathrm{mM}$ HEPES buffer pH 7.5, $5 \mathrm{mM}$ ATP, $5 \mathrm{mM} \mathrm{MgCl}$ and $0.5 \mathrm{mM}$ TCEP. The enzyme solution was identical, except that it contained $2 \mu \mathrm{M}$ HalM2 instead of His6P-HalA2. The $\mathrm{pH}$ of each solution was checked with an microtip electrode and both solutions were allowed to equilibrate for 30 min prior to mixing to initiate the reaction. After mixing, the final concentrations were $1 \mu \mathrm{M} \mathrm{HalM} 2$, $50 \mu \mathrm{M}$ His6P-HalA2, $100 \mathrm{mM}$ HEPES ( $\mathrm{pH}$ 7.5), $5 \mathrm{mM}$ ATP, $5 \mathrm{mM} \mathrm{MgCl} 2$ and $0.5 \mathrm{mM}$ TCEP. Unless otherwise noted, these standard conditions were used for all HalM2/HalA2 activity assays reported herein.

Assay Work-up: After initiation of the standard HalM2/HalA2 reaction, $50 \mu \mathrm{L}$ aliquots were removed at the desired time points and quenched by addition of $450 \mu \mathrm{L}$ of $100 \mathrm{mM}$ Sodium Citrate ( $\mathrm{pH} 3.0), 1 \mathrm{mM}$ EDTA. The quenched time points were stored at $-20{ }^{\circ} \mathrm{C}$ until the completion of the reaction. After all time points had been quenched, TCEP was added to each individual aliquot to a final concentration $10 \mathrm{mM}$ and the mixture incubated at $37{ }^{\circ} \mathrm{C}$ for $10 \mathrm{~min}$. The $\mathrm{pH}$ of the reaction mixture was adjusted to $\mathrm{pH} \sim 6.2$ by the addition of approximately $20 \mu \mathrm{L}$ of $5 \mathrm{M} \mathrm{NaOH}$, the mixture was spiked with $5.5 \mu \mathrm{L}$ of $1 \mathrm{M}$ $\mathrm{N}$-etheylmaleimide (in EtOH) to give a final concentration of $10 \mathrm{mM} \mathrm{NEM}$. The alkylation reaction was allowed to proceed at $37{ }^{\circ} \mathrm{C}$ for $10 \mathrm{~min}$. After $10 \mathrm{~min}$, the reaction was quenched by spiking the samples with concentrated trifluoroacetic acid (TFA) to give a final concentration of 1\% TFA. Individual quenched reaction aliquots were then desalted using $1 \mathrm{~mL} \mathrm{C}_{4}$-solid phase extraction cartridges (Chromabond, product number 730225 ). The column was activated using $2 \mathrm{CV} 40 \%$ acetonitrile, $0.1 \%$ TFA in water. After activation, the column was equilibrated with $4 \mathrm{CV}$ of $0.1 \%$ TFA in $\mathrm{H} 2 \mathrm{O}$. The sample ( $550 \mu \mathrm{L}$ ) was then loaded onto the SPE column, desalted with another $4 \mathrm{CV}$ of $0.1 \%$ TFA in $\mathrm{H}_{2} \mathrm{O}$, then eluted with $1.4 \mathrm{CV}$ of $80 \% \mathrm{ACN}, 0.1 \%$ TFA solution. The eluent was flash frozen in liquid nitrogen and lyophilized to dryness. 
LC-ESI-MS Analysis of Kinetic Assay Samples: After lyophilization, the NEM-alkylated peptide samples were dissolved in $100 \mu \mathrm{L}$ of $0.1 \%$ formic acid $(F A)$ in $\mathrm{H}_{2} \mathrm{O}$ and were separated using a Waters ACQUITY BEH C 8 column $(1.7 \mu \mathrm{m}, 2.1 \mathrm{~mm} \times 100 \mathrm{~mm})$ and a water:acetonitrile:formic acid solvent system consisting of $0.1 \% \mathrm{FA}$ in water (solvent $A$ ) and $0.1 \% \mathrm{FA}$ in acetonitrile (solvent B). Unless otherwise indicated, this solvent system was used for all LC-MS analyses reported in this study. Following injection of a $15 \mu \mathrm{L}$ sample (of approximately $25 \mu \mathrm{M}$ total peptide), the peptides were separated at a flow rate of $50 \mu \mathrm{L} / \mathrm{min}$ using a linear gradient of $3-100 \%$ solvent B over $10 \mathrm{~min}$. Under these conditions, the various post-translationally modified forms of His6P-HalA2 elute over a time window from approximately 4.5-6.0 min. For all kinetic assays, samples were injected in a randomized order to prevent propagation of systematic errors. Following each sample injection, blank injections were performed to ensure that peptide carry-over was minimal between individual runs. The eluent from the column was directed to the electrospray ionization (ESI) source of a Waters Synapt G2-Si hybrid quadrupole time-offlight mass spectrometer. Unless otherwise noted, the following source settings were used for all LC-ESI-MS analyses: polarity = positive ion mode, resolution mode, capillary voltage $=3.00 \mathrm{kV}$, cone voltage $=40 \mathrm{~V}$, source offset $=80 \mathrm{~V}$, cone gas $=50 \mathrm{~L} / \mathrm{h}$, desolvation gas $=600 \mathrm{~L} / \mathrm{h}$, source temperature $=100{ }^{\circ} \mathrm{C}$, desolvation temperature $=250$ ${ }^{\circ} \mathrm{C}$. Mass spectra spanning the $\mathrm{m} / \mathrm{z}$ range $100-2000 \mathrm{~m} / \mathrm{z}$ were collected (1 scan per second) in centroid mode, and glu-1-fibrinopeptide B (Glu-Fib) was used as the lockmass (external standard) to correct all $\mathrm{m} / \mathrm{z}$ values.

Determining the relative abundance of HalM2/HalA2 reaction intermediates: Prior to the kinetic modeling of HalM2 and its mutant enzymes described in the Results and Discussion section, the relative fractional abundances of each HalA2-derived intermediate had to be extracted from the raw LC-ESI-MS data. Several software tools were employed to streamline this laborious process as much as possible. First, the QuanLynx module of MassLynx software (Waters) was used to generate extracted ion chromatograms (EICs) for each ion of interest in each kinetic assay time point. The method was designed to generate and integrate an EIC for the three most abundant isotope peaks for each peptide of interest in the $8^{+}, 9^{+}$and $10^{+}$charge states. The 
integrated EIC peak areas were all manually inspected and adjusted if necessary, in order to capture the chromatographic peak associated with each signal. The data were then exported from MassLynx and processed using an in-house Matlab script that calculates a charge state weighted fractional abundance for each HalA2-derived species from the processed EIC data as described previously. ${ }^{2}$ After the data was processed, the fractional abundances of the intermediates were summated based on their dehydration and cyclization state. For simulating the dehydration data for the HalM2GS389-396 enzyme, phosphorylated intermediates were grouped according to the number of dehydrated residues they possessed (i.e. a peptide containing 1 dehydration and 1 phosphate moiety would be grouped with other singly-dehydrated peptides).

General procedure for simulations: After summing the fractional abundances of HalA2 intermediates on the basis of their dehydration or cyclization, the data were imported in Kintek Explorer for numerical simulation. ${ }^{3,4}$ The standard deviation in the data was estimated by fitting the concentration vs. time trajectories for each HalA2 peptide species to an appropriate exponential function using the "aFit" tool in Kintek Explorer. These standard deviations were then used to normalize the residuals obtained from the global least squares fit of the data to various kinetic models (discussed below) to obtain a reduced chi square $\left(\chi^{2} / D O F\right)$ value that was used to assess the goodness of fit. All simulations utilized starting concentrations for HalA2 and enzymes of $50 \mu \mathrm{M}$ and $1 \mu \mathrm{M}$, respectively, which were determined spectrophotometrically using the extinction coefficients at $280 \mathrm{~nm}$ for these molecules calculated from the peptide/protein amino acid sequence using the ExPASy ProtParam tool. Unless otherwise stated, all simulations were carried out using HalA2 binding affinities for each enzyme measured using the fluorescence polarization assay (Figure S1). It has been previously demonstrated for the HalM2:HalA2 interaction that the leader peptide (which is not post-translationally modified by HalM2) provides most of the binding affinity. ${ }^{5}$ Thus, we assumed that all HalA2 intermediates generated in the reaction mixtures had identical binding affinities for HalM2. For initial values, the dissociation constant $\left(k_{\text {off }}\right)$ was set to a value of $15 \mathrm{~min}^{-1}$ (the value for $k_{\text {off }}$ measured previously for binding of wt HalM2 to HalA2) ${ }^{2}$ and the value of $k_{\text {on }}$ was then adjusted to provide the dissociation constant measured in the fluorescence 
polarization assay. During the simulations, the magnitudes of $k_{o n}$ and $k_{o f f}$ were allowed to vary, while holding their ratio constant. Unless otherwise stated, all dehydration and cyclization steps were assumed to be irreversible and were allowed to vary freely during the global fit. In some cases, the standard errors and FitSpace calculations suggested that certain parameters were not well constrained by the data. In these cases, we exploited the real time dynamic simulation capabilities of Kintek Explorer to estimate lower limits for the unconstrained rate constants, and then fixed the relative rates of the unconstrained parameter to a more highly constrained parameter in the model and varied the two as a single parameter in the fit.

Simulation of kinetic models for dehydration: The dehydration reactions were simulated as a sequential set of reactions with irreversible chemical transformations and identical binding affinities for each dehydrated peptide state. Using the $K_{d}$ values determined from the fluorescence polarization assays, the magnitudes of the binding rates ( $k_{o n}$ and $k_{\text {off }}$ ) were initially allowed to vary freely (while holding the ratio constant) along with the rate constants for each of the chemical transformation steps. These fits, however, led to unconstrained values for the first dehydration rate $\left(k_{d, 0 \rightarrow 1}\right)$. Therefore, after estimation of $k_{d, 0 \rightarrow 1}$ with initial fits, this parameter was linked to $k_{o n}$ and $k_{o f f}$. In general, each of the other apparent dehydration rates were well constrained by the data, and these parameters were allowed to vary freely in the fit. An additional step, $\left(k_{\text {inact }}\right)$, was included in the mechanisms for all of the enzymes except for wt HalM2 and HalM2P405A to account for an apparent slow loss of dehydration activity. We were able to simulate the dehydration data without the $k_{\text {inact }}$ parameter if we assumed that the dehydration reactions were reversible, but we consider this mechanism unlikely given the highly exergonic dehydration reaction (which consumes ATP). Thus, we did not consider this model further. It is possible that the variant enzymes may be inhibited by some of the numerous species that form in the in vitro reaction mixture. For the HalM2Gs389-386 enzyme, an alternative dehydration model was needed in order to account for the numerous phosphorylated intermediates that accumulated in the reaction (Figure S3 and S11). 
Simulation of kinetic models for cyclization: The cyclization data for each HalM2 enzyme was simulated using the $k_{o n}$ and $k_{o f f}$ values determined in the fits of the dehydration data for that enzyme. Similar magnitudes for $k_{o n}$ and $k_{o f f}$ were obtained in fits of the cyclization data if we allowed these parameters to vary while holding the $K_{d}$ constant. As we observed when fitting the dehydration data, the rate constant for the first cyclization step $\left(k_{c, 0 \rightarrow 1}\right)$ was not well-constrained for any of the enzymes, so this parameter was linked with the binding rates. We initially attempted to simulate the installation of the four thioether rings into HalA2 using a sequential reaction with irreversible chemical transformations. However, for wt HalM2 and the HalM2P349A, HalM2P405A, and HalM2T386A/D387A variants, the fourth and final cyclization was fast and was not well constrained by the data. Initial fitting produced unreasonably large values for the $k_{c, 3 \rightarrow 4}$ rate constant; thus, we fixed the value of this parameter at 10x the magnitude of $k_{c, 2 \rightarrow 3}$ (which did not significantly change the appearance of the fitted curves) and simulated the pair of linked rate constants as a single variable parameter. This data implies that the final two cyclizations in these enzymes are strongly coupled and that $k_{c, 3 \rightarrow 4} \gg k_{o f f}$ for 3-fold cyclized intermediates. For the HalM2 ${ }_{G S 635-644}$, HalM2 ${ }_{G S 491-497}$, and HalM2GS389-396 variants, the rate of the $4^{\text {th }}$ cyclization was considerably slower relative to $k_{c, 2 \rightarrow 3}$ and $k_{o f f}$, and could be adequately simulated as a separate parameter.

Using these initial models, we were unable to simulate the small amounts of 3 -fold cyclized intermediates that accumulated and persisted in the wt HalM2, HalM2P405A, and HalM2T386AID387A reactions. Thus, we made several adjustments to the kinetic mechanism to better account for these species (cyclization models 1-3, Figure S4). The results of these fits are summarized in Table S3. In model 1, we allowed the cyclization steps to be reversible. While the addition of thiols to $\alpha, \beta$-unsaturated ketones and other Michael acceptors is generally exergonic and favors the adduct, ${ }^{6}$ it has recently been shown that HalM2 is capable of opening each of the 4 thioether rings in mature HalA2. ${ }^{7}$ For wt HalM2, HalM2P405A, and HalM2T386A/D387A, excellent fits to the data could be obtained by allowing the $4^{\text {th }}$ cyclization to be reversible. For the wt and HalM2T386A/D387A enzymes, the value of the reverse rate constant $\left(k_{c, 4 \rightarrow 3}\right)$ was well constrained and was allowed to vary freely in 
the fit. For HalM2 ${ }_{\mathrm{P} 405 \mathrm{~A}}, k_{c, 4 \rightarrow 3}$ was linked to $k_{c, 3 \rightarrow 4}$ after its initial value had been simulated.

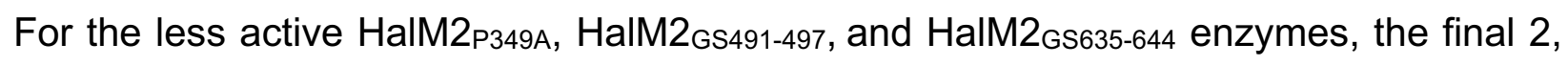
3 , and 4 cyclizations, respectively, required reversible steps. For these reactions, the reverse rate constants were initially freely varied, and were then fixed relative to each other and simulated as single variable parameter. The results from the fits of the cyclization data to model 1 are reported in Table 1 (for wt HalM2, HalM2P405A, and HalM2T386A/D387A), Table S3 (for HalM2P349A, HalM2GS491-497, and HalM2GS635-644) and in Figures S5-S7.

In model 2, the partially cyclized HalA2 intermediates that were not fully consumed in the reaction were treated as dead-end side products. This was deemed to be the most appropriate model for the HalM2GS389-396 variant for several reasons. First, the reaction spectra for this enzyme clearly show the accumulation of phosphorylated, partially cyclized intermediates that are not consumed (even after overnight incubation). Under the reaction conditions employed, phosphate elimination from HalA2 intermediates released by the enzyme is expected to be very slow. ${ }^{2}$ This is because phosphate elimination also requires binding of ADP to the dehydratase active site, yet ATP is present in the reactions in far higher concentrations. It is also possible that the phosphate moieties on the HalA2 core peptide inhibit binding of the core peptide to the cyclase active site. In addition, MSMS analysis of some of the phosphorylated species generated by HalM2GS389-396 localized the phosphate to the Ser11 and Thr17 residues that are directly involved in the formation of rings $\mathrm{B}$ and $\mathrm{C}$, respectively. Phosphorylation at these positions would thus be expected to directly prevent subsequent cyclization. We also favor model 2 for the HalM2P349A, HalM2 ${ }_{G S 491-497}$, and HalM2 ${ }_{G S 635-644}$ enzymes because each of these enzymes generates partially cyclized HalA2 intermediates that persist in the reaction mixture (Figures S8-S10) and that appear to have unusual (non-native) thioether topologies (Figure S25). The results from the fits of the cyclization data to model 2 are reported in Table 1 (for HalM2P349A, HalM2GS491-497, and HalM2GS635-644), Table S3

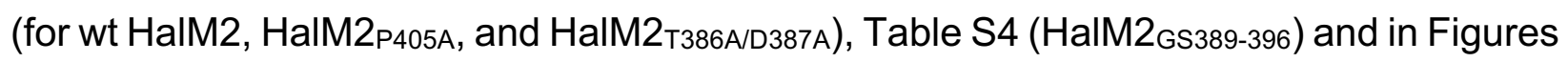
S8-S11. 
In model 3, we kept the cyclization reactions irreversible and assumed that the partially cyclized peptides were catalytically competent intermediates with altered binding affinities to the enzyme. For all of these fits, the equilibrium binding constant $\left(K_{d}\right)$ for the unmodified HalA2 peptide was equivalent to the value measured for each variant enzyme using the FP assay. The binding constants for peptides containing thioether rings were then modified as needed by allowing $k_{\text {on }}$ to vary. In our initial simulations of this model, we found that it was not generally possible to achieve good fits by varying $k_{o f f}$, so this parameter was held constant for all species. Thus, in this model, the accumulation of partially cyclized intermediates results from reduced second order binding rates to the

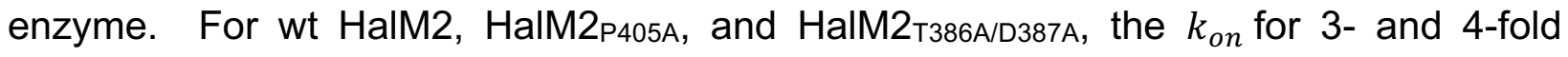
cyclized species was varied. For HalM2P349A, the $k_{\text {on }}$ values for 2-, 3-, and 4-fold cyclized species were varied, and for HalM2 ${ }_{\mathrm{GS} 491-497}$ and HalM2 ${ }_{\mathrm{GS635-644}}$, the binding rates for all cyclized species had to be altered. While these fits resulted in low $\chi^{2} / D O F$, the simulated $k_{\text {on }}$ values for each enzyme assumed a range values spanning 2-3 orders of magnitude. Given the known strong contribution of the leader peptide to the binding affinity, it is difficult to rationalize how the rebinding rates for partially cyclized intermediates could be so drastically different. For this reason, we currently do not favor this model.

Tandem MS experiments: Kinetic assay time points from the various enzyme-catalyzed reactions were chosen for MSMS analysis based on the abundance of the desired species and were lyophilized to dryness. The samples were resuspended and digested in a $50 \mu \mathrm{L}$ solution containing 1 unit of GluC protease (Promega) in $10 \mathrm{mM}$ phosphate buffer $(\mathrm{pH} 7.0)$ for $1 \mathrm{~h}$ at $37^{\circ} \mathrm{C}$. After digestion, the samples were $\mathrm{C}_{4}$ SPE purified and eluted in $80 \%$ acetonitrile. After lyophilization, digested peptide was resuspended in $0.1 \%$ formic acid for tandem MS analysis. Chromatography conditions used for tandem MS were the same as previously stated for the kinetic assays. Mass spectral data were collected under the same instrument settings used for the kinetic assay time points, except that spectra were collected in continuum mode and the scan time was reduced to $0.4 \mathrm{sec}$. The quadrupole mass filter was centered on the most abundant isotope peak of the desired species and was operated with the maximum resolution available on the instrument $(L M$ resolution $=25)$ to avoid passage of species with similar $\mathrm{m} / \mathrm{z}$ values into 
the collision cell. The selected species were fragmented by collision induced dissociation using argon as the collision gas and collision energy ramps of 20-25 V and 25-30 V applied over a $0.4 \mathrm{~s}$ interval. Fragmentation spectra were smoothed and deconvoluted using the MaxEnt3 function of MassLynx software (Waters).

Hypothetical Structure Evaluation and Enumeration (HSEE): The deconvoluted CID fragment ion mass spectra were analyzed using hypothetical structure enumeration and evaluation $(\mathrm{HSEE})^{8}$ to map the location of the HalM2-installed post-translational modifications onto the HalA2 core peptide. The inputs for HSEE are the amino acid sequence of the peptide (i.e. GluC-digested HalA2 = LSSLAGSGDVHAQTTWPCATVGVSVALCPTTKCTSQC), the number of each type of post-transitional modification present in the selected precursor ion, the mass changes associated with each type of post-translational modification, and the possible positions within the peptide where the post-translational modifications can occur. For our experiments, the input mass changes were -18 Da for dehydration of Ser/Thr, +80 Da for phosphorylation or Ser/Thr residues, and +125 Da for NEM-alkylated Cys residues. The HSEE script (executed in R) enumerates all of the possible hypothetical structures based on these input parameters and then evaluates these structures by spectral matching of predicted and observed fragment ions. An HS score is calculated for each hypothetical structure (based on the number of matched ions) allowing the most appropriate hypothetical structure to be selected in an unbiased fashion.

HDX-MS assays: The HDX reactions were prepared using the previously described workflow by our group. ${ }^{9}$ Briefly, HalM2 variants were diluted from concentrated stock solutions to a final concentration of $1 \mu \mathrm{M}$ in an $\mathrm{HDX}$ reaction containing $5 \mathrm{mM} \mathrm{MgCl}, 1$ $\mathrm{mM}$ TCEP, $50 \mathrm{mM}$ HEPES, $1 \mathrm{mM}$ AMP-PNP and $25 \mu \mathrm{M}$ of HalA2 (when present). Each sample was prepared in triplicate. Deuterated stock solutions of HEPES, TCEP, $\mathrm{MgCl}_{2}$, and $\mathrm{HalA} 2$ were prepared by repeating two cycle of resuspension of components in $\mathrm{D}_{2} \mathrm{O}$ and lyophilization to replace as many of the exchangeable protons with deuterons as possible. The HalM2 variants were concentrated to $60 \mu \mathrm{M}$ in storage buffer containing 50 $\mathrm{mM}$ HEPES, $\mathrm{pH} 7.5,100 \mathrm{mM} \mathrm{KCl}, 10 \%$ glycerol using a $0.5 \mathrm{~mL}$ Amicon Ultracel 10k 
centrifugal filtration device (Millipore). The HDX reactions were quenched after 5 min by adding $75 \mu \mathrm{L}$ of the quench buffer $(100 \mathrm{mM}$ potassium phosphate, $0.8 \mathrm{M}$ guanidine $\mathrm{HCl}$, $\mathrm{pH}$ 1.9) and by flash freezing in liquid nitrogen. The quenched HDX reaction yielded a final $\mathrm{pH}$ meter reading of 2.3 , at which the $\mathrm{H} / \mathrm{D}$ back exchange of the protein backbone amides is minimal. The samples were then stored in $-80{ }^{\circ} \mathrm{C}$ until collection of mass spectrometry data.

Mass spectrometry analysis of HDX samples: Prior to injection of samples, the Waters Synapt G2-Si mass spectrometry instrument with HDX Technology was properly calibrated. Unless otherwise noted, all steps in the mass spectral data collection were performed as previously reported..$^{9}$ The samples were thawed at $37^{\circ} \mathrm{C}$ water bath for exactly $65 \mathrm{~s}$ followed by injection into a $40 \mu \mathrm{L}$ loop at exactly 2 min after removing the sample from the $-80^{\circ} \mathrm{C}$ freezer. The sample was then passed through a Waters Enzymate $\mathrm{BEH}$ column containing immobilized pepsin at $15{ }^{\circ} \mathrm{C}$ using a $0.1 \%$ formic acid mobile phase (flow rate $100 \mu \mathrm{L} / \mathrm{min}$ ). The peptides produced by the pepsin digestion were accumulated on a C18 guard column held at $4{ }^{\circ} \mathrm{C}$ for 3 min and were desalted with $0.1 \%$ formic acid. Next, the trapped peptides were eluted onto a Waters $\mathrm{BEH} \mathrm{C}_{18}$ UPLC column (1 $\times 100 \mathrm{~mm}$, equilibrated in 97:3 (v/v) $\mathrm{H}_{2} \mathrm{O}$ : acetonitrile, $0.1 \%$ formic acid) and separated by applying a gradient of $3-100 \%$ acetonitrile $(0.1 \%$ formic acid) over $10 \mathrm{~min}$. The $\mathrm{C} 18$ guard column and analytical $\mathrm{C} 18$ column were both held at $0.4{ }^{\circ} \mathrm{C}$ in order to avoid H/D back exchange during peptide desalting and chromatographic separation. The separated peptides were then ionized using an electrospray ionization (ESI) source with the following source settings: capillary voltage of $2.8 \mathrm{kV}$, sampling cone $=30 \mathrm{~V}$, source offset $=30 \mathrm{~V}$, desolvation temperature $=175^{\circ} \mathrm{C}$, data collection: positive ion mode, "sensitivity" mode. The ionized peptides were passed to the IMS region of the instrument, where a travelling wave ion mobility separation was performed using a wave velocity of $650 \mathrm{~m} / \mathrm{s}$, a wave height of $40 \mathrm{~V}$, an IMS bias of $3 \mathrm{~V}$, and a nitrogen pressure of $3.1 \mathrm{mbar}$. Peptides exiting the IMS cell were subjected to two collision energy regimes in the transfer region of the IMS analyzer that were alternated in $0.4 \mathrm{~s}$ intervals: 1) a low energy regime (6 V) in order to preserve the precursor masses for measurement of deuterium uptake and 2) a high energy ramp (21-44 $\mathrm{V})$ to generate fragment ions for peptide identification. 
Fragmentation was achieved with argon as the collision gas. Both the precursor peptide and the fragmented ions were detected using a high resolution, orthogonal acceleration time of flight (TOF) mass analyzer. The $\mathrm{m} / \mathrm{z}$ data was corrected using a [glu-1]fibrinopeptide B (Glu-Fib) external lock mass standard.

Processing of the HDX data: The HDX peptide list was derived from wt HalM2 reference samples prepared in protiated buffer and subjected to the LC-MS workflow described above. The data were analyzed using Protein Lynx Global Server (PLGS) software (Waters). In this step, peptides derived from an in silico pepsin digestion of wt HalM2 were compared with the experimental data, and putative peptide identifications were scored based on a variety of user-defined criteria including the ppm error between the calculated and observed precursor ion $\mathrm{m} / \mathrm{z}$ values, the number of fragment ions detected for each peptide, and the correlation between the chromatographic retention times and ion mobility drift times of the precursor peptide and its matched fragment ions. The finalized peptide list was then used by DynamX 3.0 software (Waters) to search the deuterium exchanged samples for HDX peptides. At this stage, additional thresholding was applied to further trim the peptide list to only those peptides that were observed in each of the three replicate wt HalM2 reference samples, that were within $5 \mathrm{ppm}$ of the of the calculated $\mathrm{m} / \mathrm{z}$, and that produced at least 2 consecutive $\mathrm{CID}$ fragment ions. Reference samples (in $\mathrm{H}_{2} \mathrm{O}$ ) were also prepared for each HalM2 variant in protiated buffer, analyzed using the workflow described above, and used in DynamX 3.0 to calculate the relative deuterium uptake in the deuterium exchanged samples. Thus, although the peptide list was derived from wt HalM2, the deuterium uptake for each peptide generated from the HalM2 variants was calculated based on protiated reference samples for that variant. The isotopic distributions and relative deuterium exchange values were then manually inspected for every peptide in every sample for each variant to ensure accurate deuterium uptake measurements. These deuterium exchange values were then used to calculate the uptake difference values reported in the main text (Figure 8). Peptides undergoing significant exchange (defined as: $\mid$ uptake difference $\mid \geq 0.4 \mathrm{Da}$ ) for each HDX difference comparison are shown in Table S5-S6. This significance threshold was determined from a previous analysis of the error in this HDX-MS workflow for HalM2. ${ }^{1}$ 
Molecular dynamics simulations: The 3D structure of HalM2 was obtained by homology modelling using SwissModel ${ }^{10}$ with the default parameters. To construct the homology model, the X-ray crystal structure of the class II lanthipeptide synthetase CyIM protein (PDB ID: 5dzt) was used as the template structure. The enzyme was hydrogenated by taking into consideration the environment of the protein. Each histidine imidazole side chain was protonated according to its local polar environment. The tleap module of AMBER16 was used to build the topology and coordinate files. ${ }^{11}$ The enzyme was solvated with the addition of 61169 TIP3P water molecules. The AMBER16 program ${ }^{12}$ with the CUDA-enable graphics processing unit (GPU) version of pmemd ${ }^{13}$ was used for all MD simulations. Protein and water molecules were described using the $\mathrm{ff}^{14 \mathrm{SB}^{14}}$ and TIP3P ${ }^{11}$ force fields, respectively. Periodic boundary conditions and an NVT ensemble were applied, ${ }^{15}$ with a cut-off of $8 \AA$ being applied in real space for long-range electrostatic interactions using the Particle-Mesh Ewald (PME) procedure. A timestep of $2 \mathrm{fs}$ was used for both equilibration and production MDs through restricting the motion of hydrogens via the SHAKE algorithm. ${ }^{16}$ Due to the use of the homology model as the starting structure, steric clashes were found to be more prevalent, as expected. To remove these clashes, several alternate minimization and equilibration MDs were performed. More specifically, equilibration MDs were performed via the following five-step process: (1) To ensure the proper hydrogen positions, all heavy atoms were restrained with a $50 \mathrm{kcal} \cdot \mathrm{mol}^{-1} \cdot \AA^{-2}$ harmonic potential for $100 \mathrm{ps}$, at $10^{\circ} \mathrm{K}$; (2) To ensure optimized water positions, the simulation was further run for an additional 100 ps, without restraining water molecules; (3) To begin relaxing the protein in preparation for simulation, the simulation was run for another $100 \mathrm{ps}$, where the harmonic restraint on protein heavy atoms was relaxed to 5 $\mathrm{kcal} \cdot \mathrm{mol}^{-1} \cdot \AA^{-2}$; (4) To continue the process, the simulation was run for an additional 100 ps without restraints; (5) Finally, the simulation was gradually heated to $300{ }^{\circ} \mathrm{K}$ over a time period of 2000 ps. The velocities were randomly updated every 10 steps for equilibration stages $1-4$ and every 100 steps for stage 5 . At this point a production run of $1 \mu \mathrm{s}$ was performed to investigate the stability and the dynamics of the HalM2 protein. Finally, a cluster analysis was performed on the conformations of HalM2 using the MMTSB toolset ${ }^{17}$ and a radius of 2.5 , which identified 7 distinct clusters. It should be 
noted that only conformations between 300 to 1000 ns were considered as these were better equilibrated. For each cluster, a representative (centroid) structure was extracted. PDB files of these representative structures are provided as Supporting Materials. The time-evolution of these structures were analyzed by observing the pairwise RMSD and RMSF per residue between clusters (Figure S42). 


\section{Supporting Results}
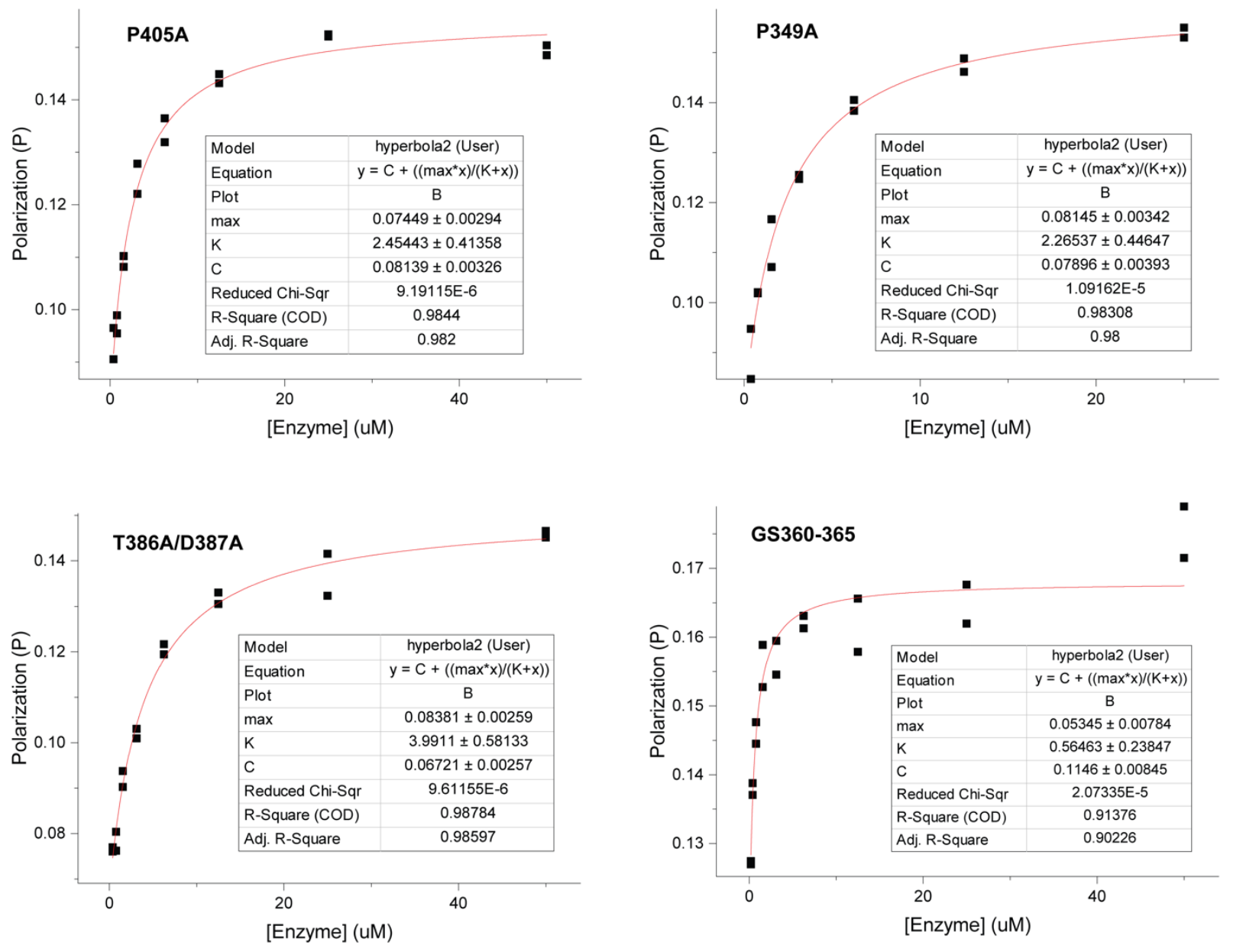

Figure S1. Fluorescence polarization binding assays were used to measure the $K_{d}$ for the interaction between the HalM2 variants constructed in this study with fluoro-HalA2LP. The data were fitted with Equation 2. The dissociation constants for wt HalM2 and the other variants employed in this study were reported previously. ${ }^{1}$ 

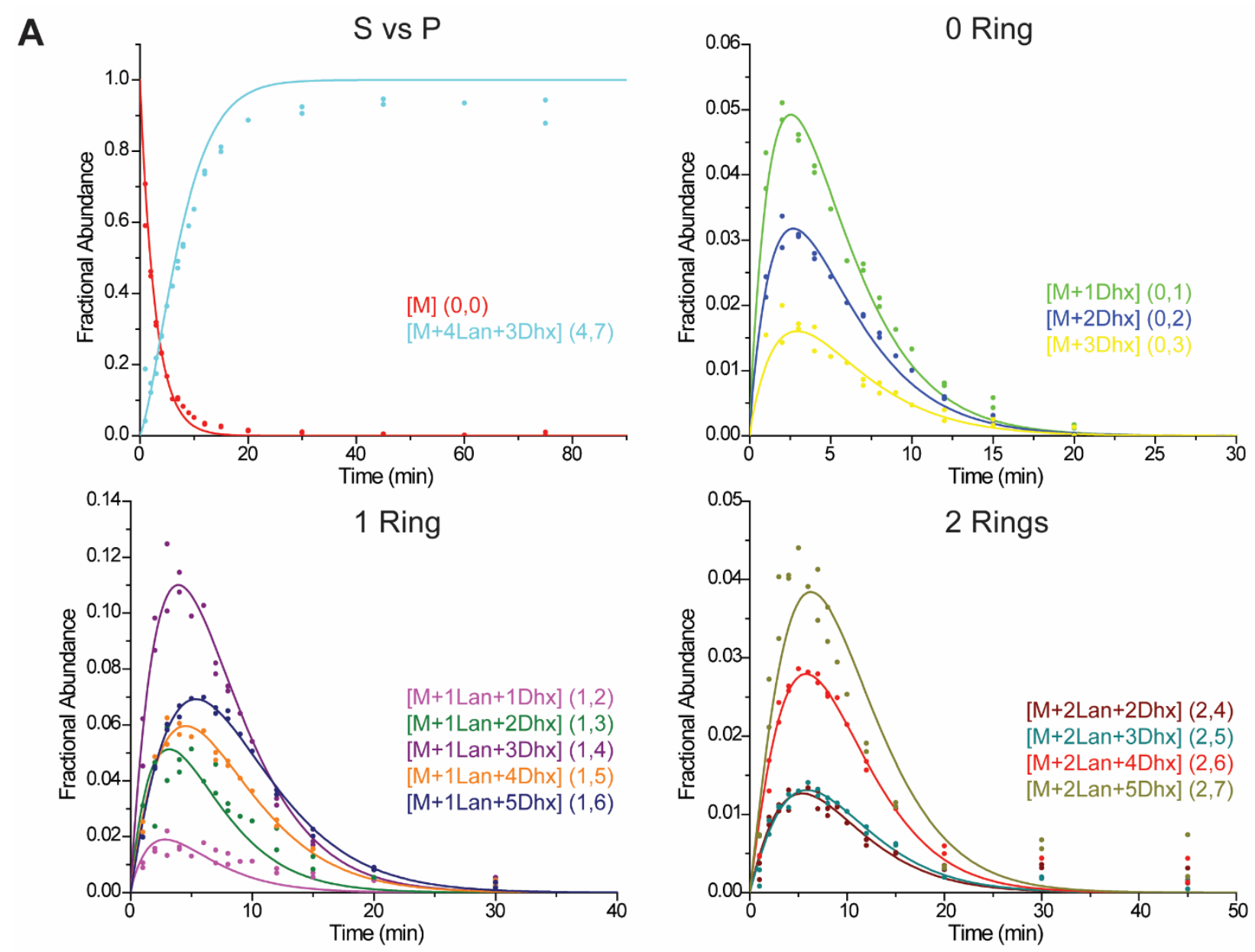

B

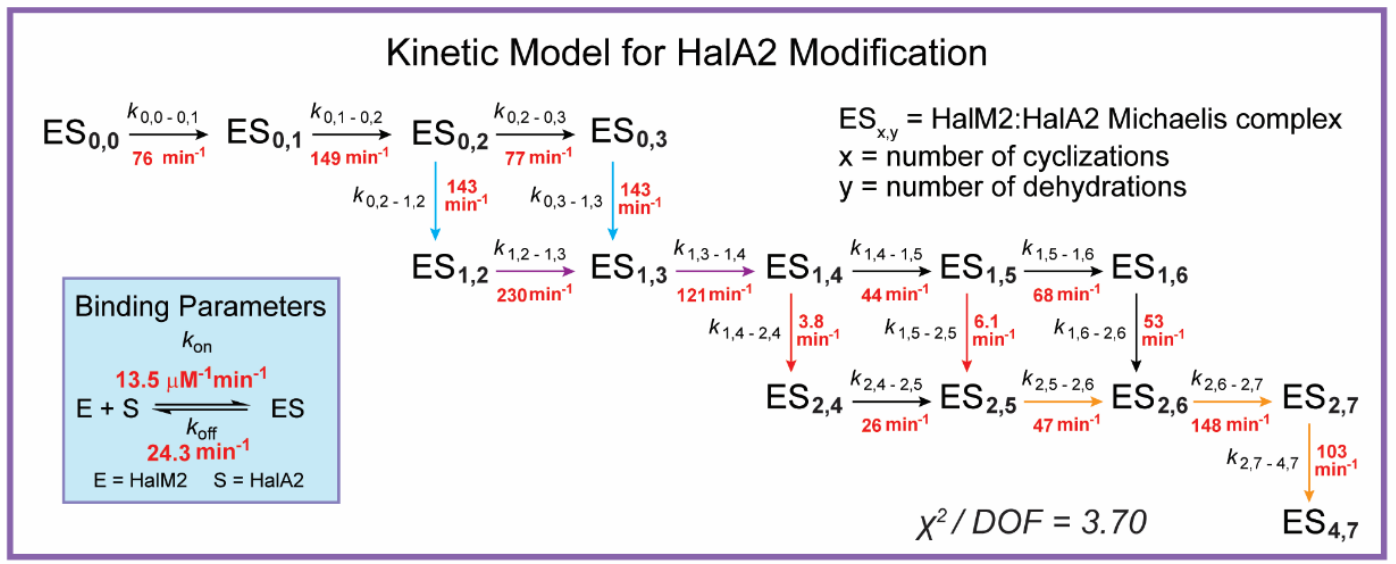

Figure S2. A) Simulated kinetic data for the wt HalM2 reaction. The kinetic data for the wt HalM2 were simulated using the previously reported model shown in panel B. ${ }^{2}$ The colored arrows represent reaction steps which were linked during the simulation. Fitting of the kinetic data resulted in a set of rate constants that were similar to those reported previously for the wt enzyme (Table S2). ${ }^{2}$ 
Kinetic Models for Dehydration

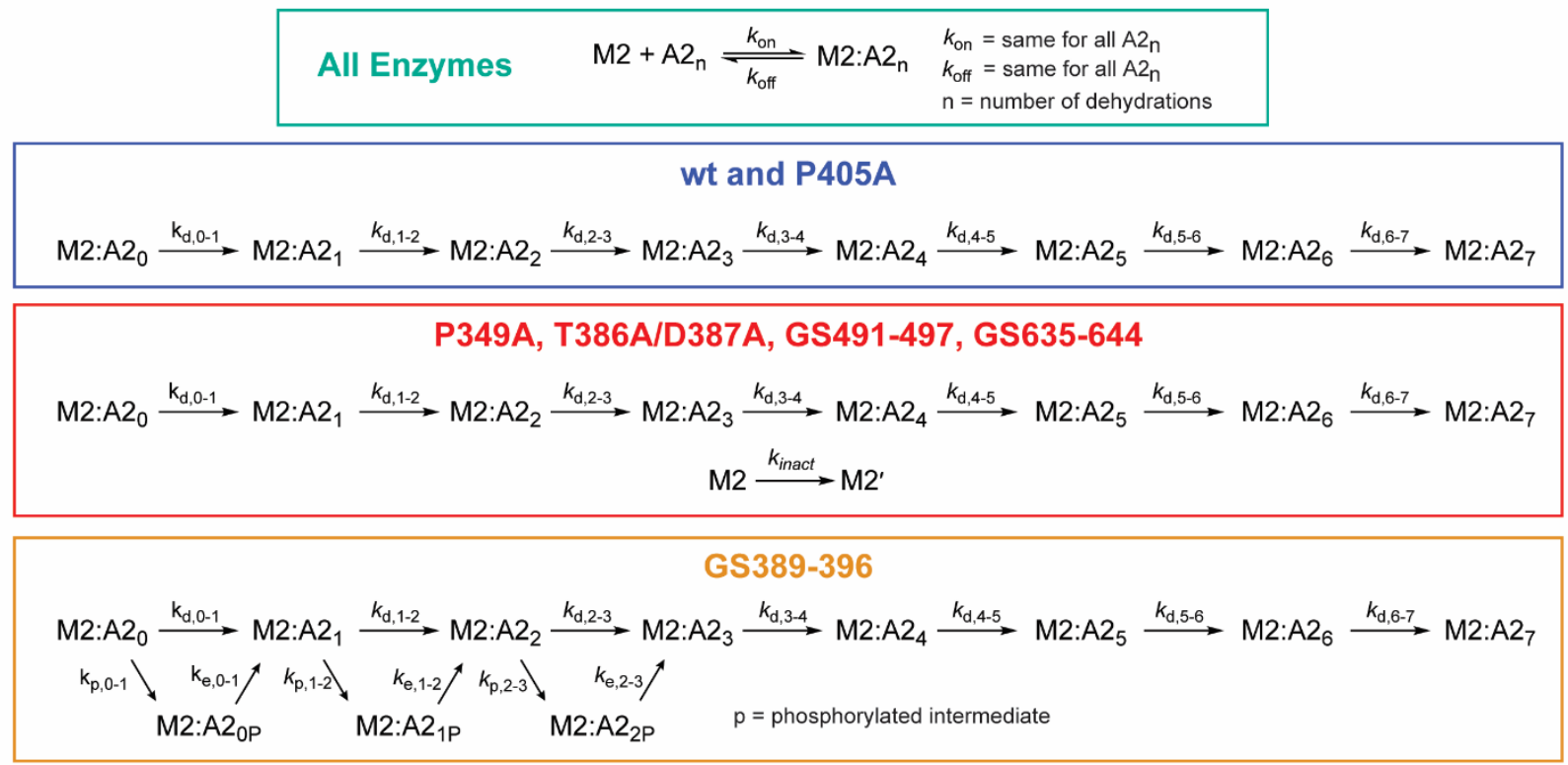

Figure S3. Models used for the numerical simulation of the kinetic data for the apparent dehydration reactions catalyzed HalM2 and its variant enzymes. For each enzyme, all partially dehydrated intermediates were assumed to have identical binding kinetics. The $K_{d, \text { HalA2 }}$ for each enzyme was determined by the fluorescence polarization assay (Figure S1 and reference 1) and this value was held constant during the global fit. With the exception of the HalM2 $2_{\text {GS389-396 }}$ variant, the dehydration data for all enzymes could be simulated with a simple, sequential dehydration model. The generation of phosphorylated intermediates by the HalM2 ${ }_{G S 389-396}$ variant required an altered kinetic model. The fits for many of the variants could be substantially improved by including a slow enzyme inactivation step: $M 2 \rightarrow M 2^{\prime}$. The simulated dehydration data for each enzyme are shown in Figures S5-S11. The fitted parameter values are provided in Tables 1 and S4. 


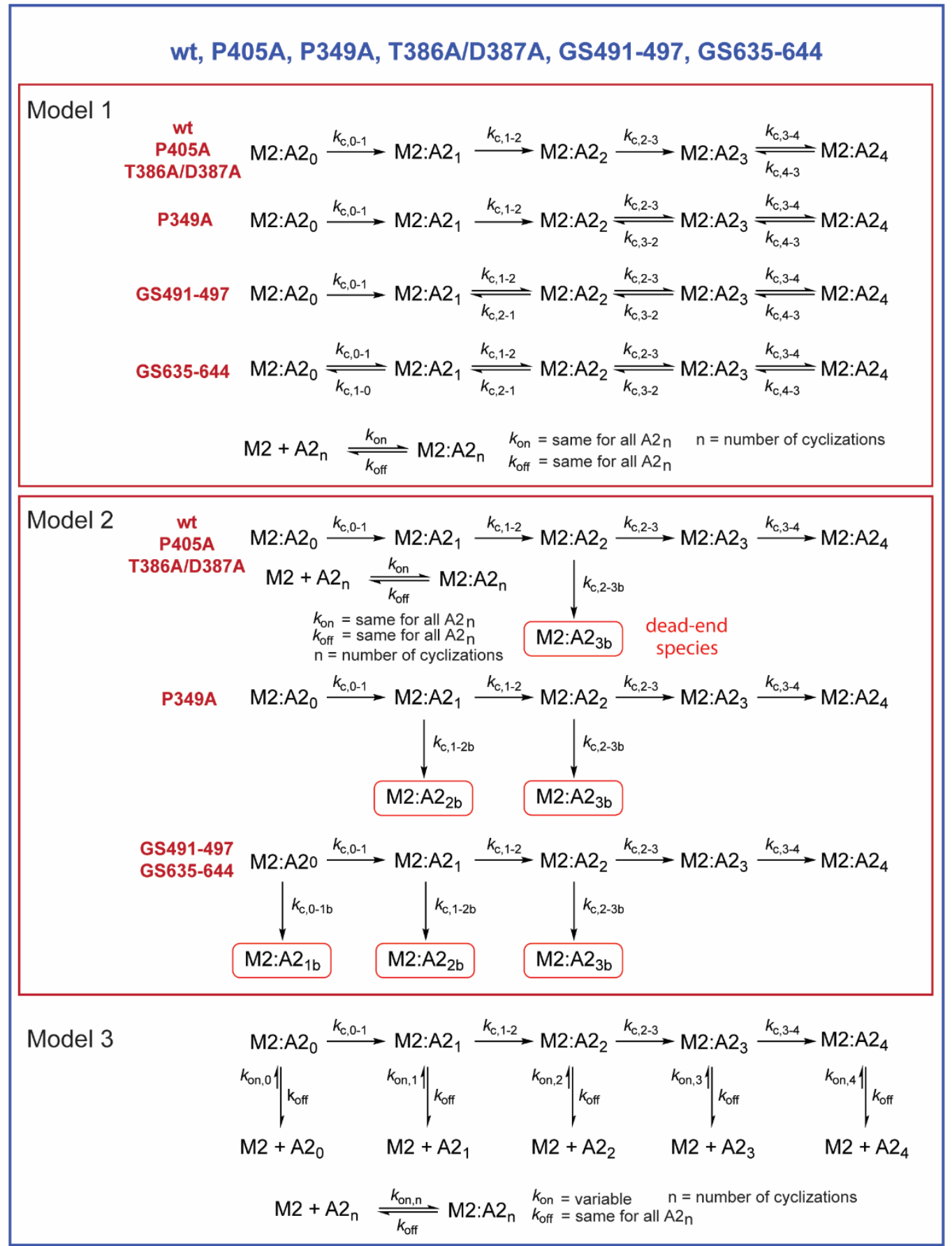

GS389-396

$\mathrm{M} 2: \mathrm{A}_{0} \stackrel{k_{\mathrm{c}, 0-1}}{\longrightarrow} \mathrm{M} 2: \mathrm{A} 2_{1} \stackrel{k_{\mathrm{c}, 1-2}}{\longrightarrow} \mathrm{M} 2: \mathrm{A} 2_{2} \stackrel{k_{\mathrm{c}, 2-3}}{\longrightarrow} \mathrm{M} 2: \mathrm{A}_{3} \stackrel{k_{\mathrm{c}, 3-4}}{\longrightarrow} \mathrm{M} 2: \mathrm{A}_{4}$

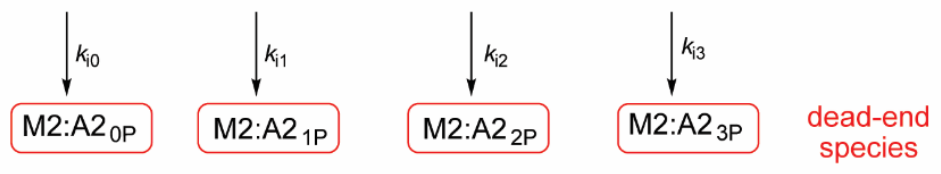

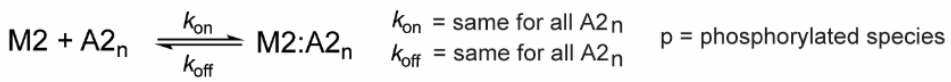


Figure S4. Models used for the numerical simulation of the kinetic data for the cyclization reactions catalyzed by HalM2 and its variant enzymes. The data for all enzymes (with the exception of HalM2GS389-396) were simulated with models 1-3 as described in more detail in the Supporting Information. Data could be fitted with reversible cyclization steps (model 1), by treating the partially cyclized intermediates as dead-end species (model 2), or by altering the binding kinetics of the partially cyclized intermediates (model 3 ). The simulated cyclization data are shown in Figures 3, S5-S11 and the fitted parameter values are compared in Tables 1, S3, and S4. 


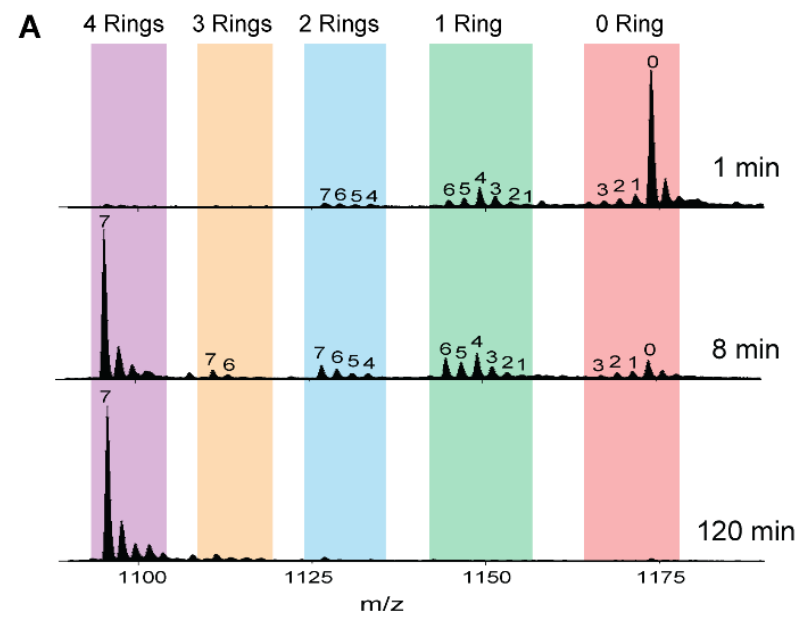

B

C

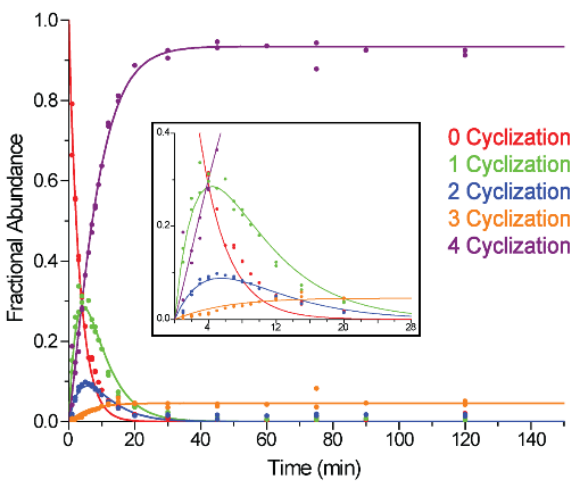

E

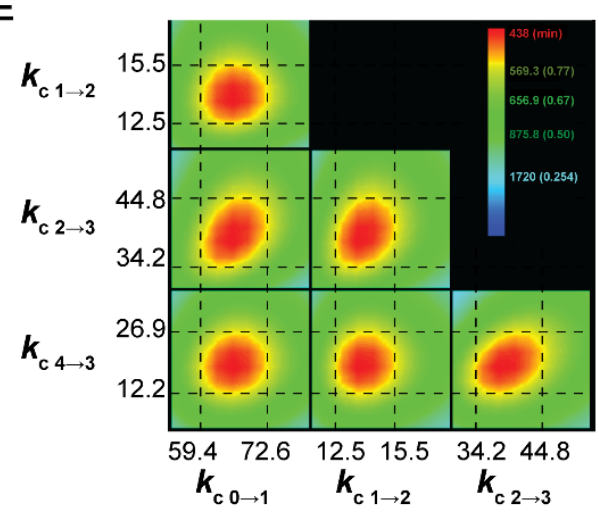

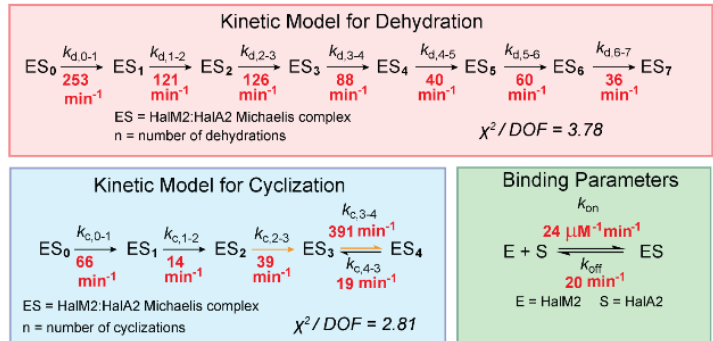

D

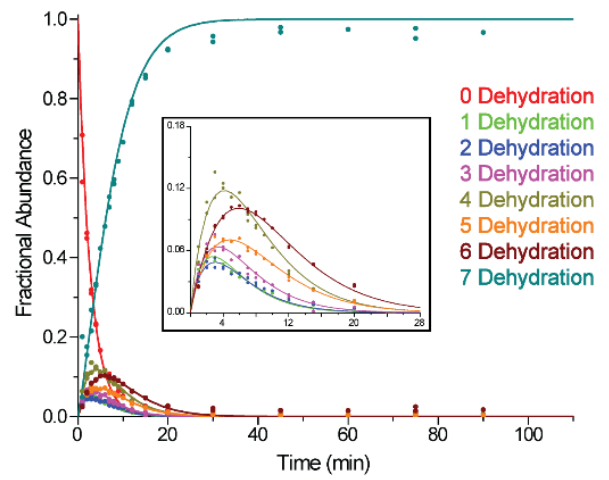

F

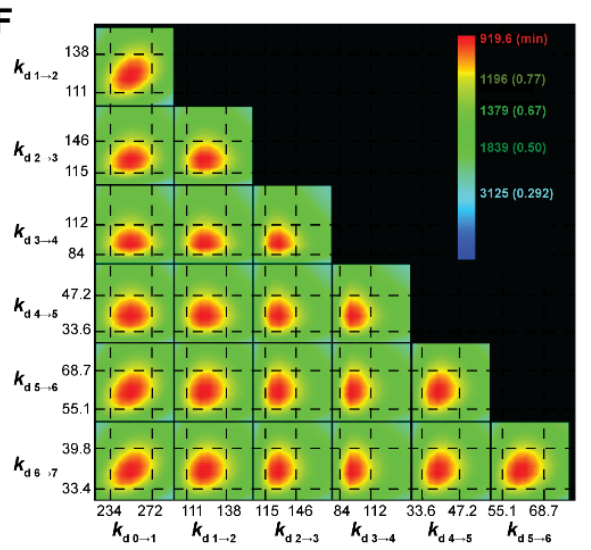

Figure S5. Simulated kinetic data for wt HalM2. A) LC-ESI-MS spectra of the wt HalM2/HalA2 reaction mixture shown as a function of reaction time. The $8^{+}$charge state is shown. The numbering above the peaks indicates the number of dehydrated Ser/Thr residues in the intermediate. Peptides have been NEM-alkylated as described in the Supporting Methods to reveal the number of thioether rings. B) Kinetic models and 
binding parameters used for the simulation of dehydration and cyclization kinetic data (see also Figures S3-S4). Colored arrows represent apparent rate constants that were linked during the simulation. The goodness of fit is indicated by the $\chi^{2} / D O F$ statistic. Kinetic data for cyclization (panel C) and dehydration (panel D) simulated with the models in (B). The fractional abundances of species containing the same number of dehydrations/cyclizations were summed prior to simulation as described in the supporting methods. E and F) FitSpace calculations for the cyclization and dehydration data, respectively, were used to estimate variable kinetic parameter boundaries at a threshold of 1.2 times the $\chi^{2}$ minimum of the global fit (the red portion of each individual sub-plot). Rate constants are in units of $\mathrm{min}^{-1}$. All fitted parameter values and their corresponding FitSpace boundaries are reported in Table 1 of the main text. 


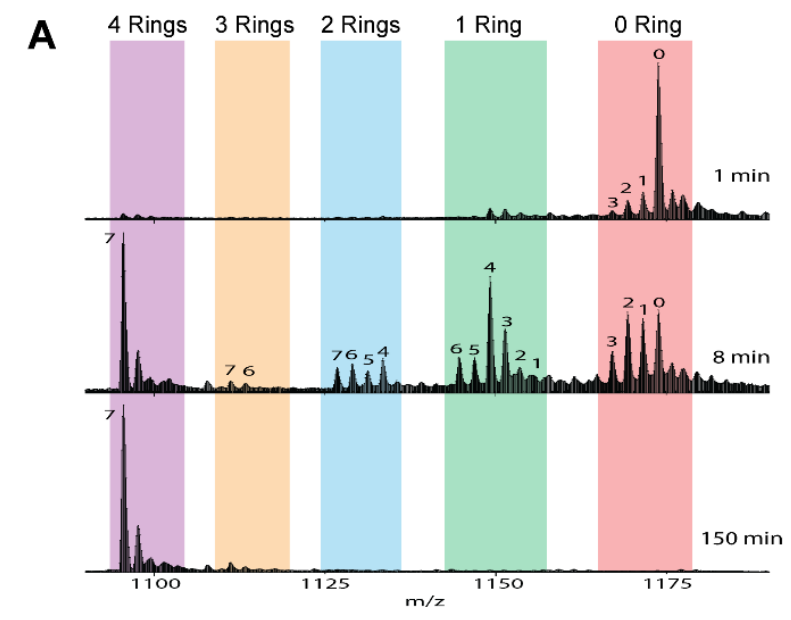

B
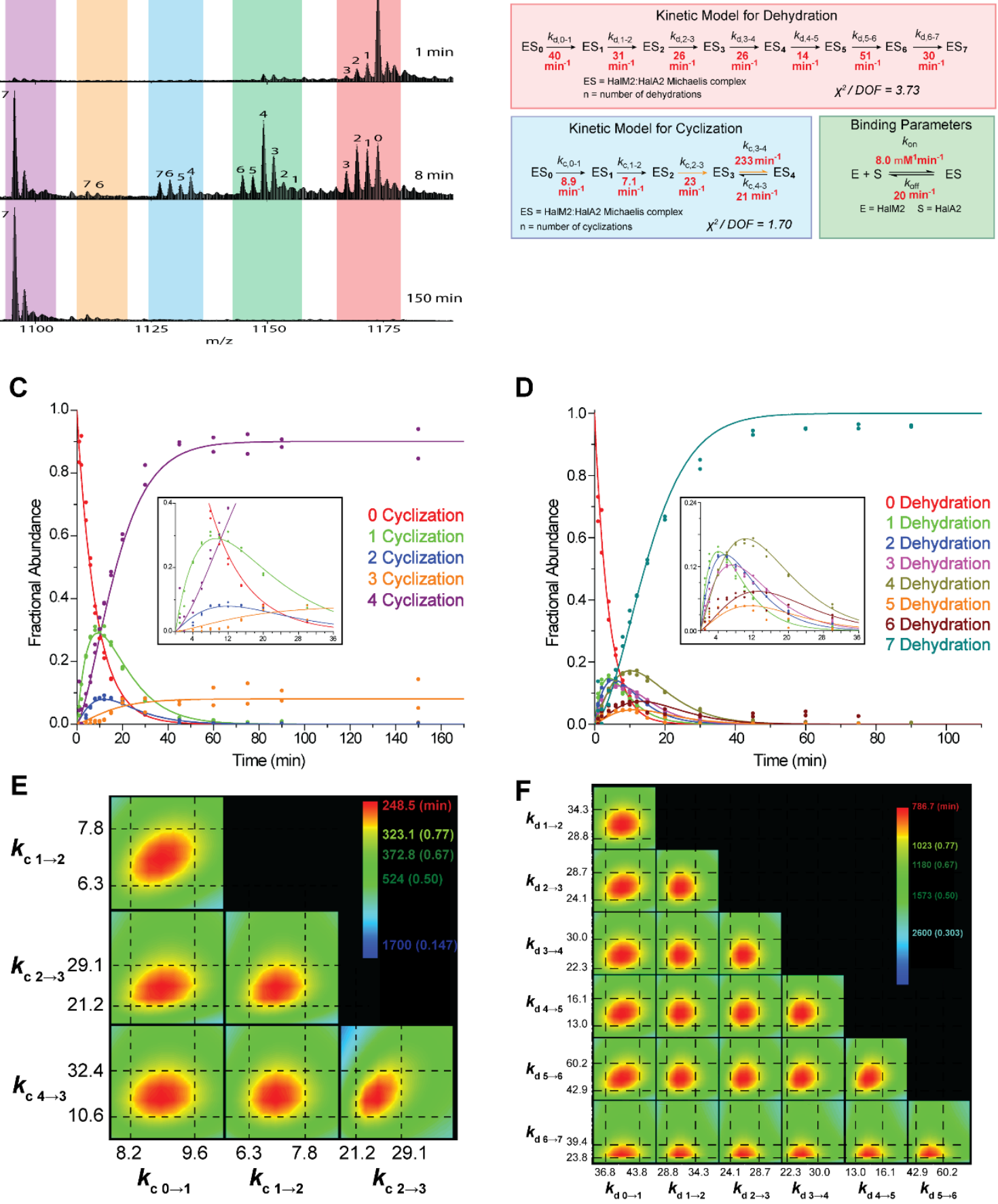

Figure S6. Simulated kinetic data for HalM2p405A. A) LC-ESI-MS spectra of the HalM2 ${ }_{\mathrm{P} 405 \mathrm{~A}} / \mathrm{Hal} \mathrm{A} 2$ reaction mixture shown as a function of reaction time. The $8^{+}$charge state is shown. The numbering above the peaks indicates the number of dehydrated 
Ser/Thr residues in the intermediate. Peptides have been NEM-alkylated as described in the Supporting Methods to reveal the number of thioether rings. B) Kinetic models and binding parameters used for the simulation of dehydration and cyclization kinetic data (see also Figures S3-S4). Colored arrows represent apparent rate constants that were linked during the simulation. The goodness of fit is indicated by the $\chi^{2} / D O F$ statistic. Kinetic data for cyclization (panel C) and dehydration (panel D) simulated with the models in (B). The fractional abundances of species containing the same number of dehydrations/cyclizations were summed prior to simulation as described in the supporting methods. The HalM2P405A data could be simulated using the same mechanisms that were used for wt HalM2. E and F) FitSpace calculations for the cyclization and dehydration data, respectively, were used to estimate variable kinetic parameter boundaries at a threshold of 1.2 times the $\chi^{2}$ minimum of the global fit (the red portion of each individual sub-plot). Rate constants are in units of $\mathrm{min}^{-1}$. All fitted parameter values and their corresponding FitSpace boundaries are reported in Table 1 of the main text. 
A

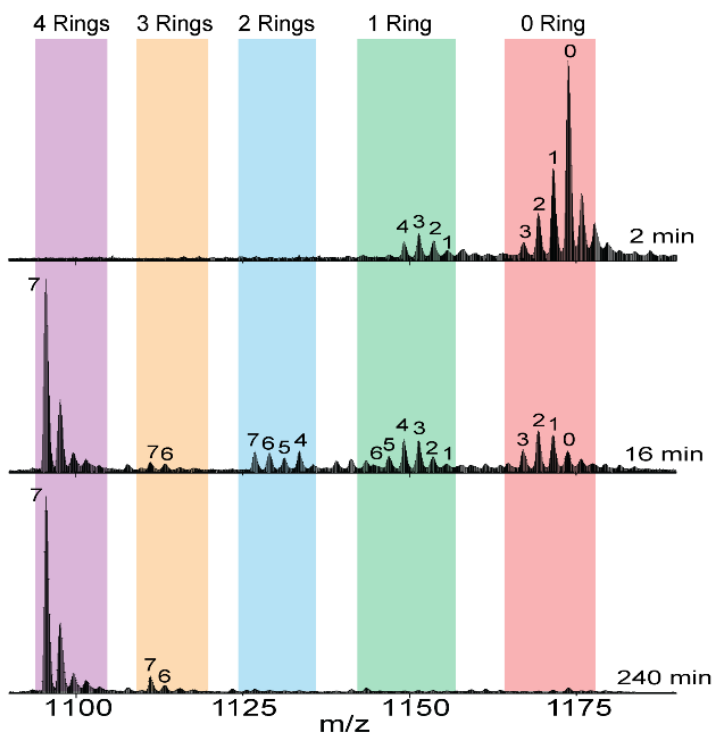

C

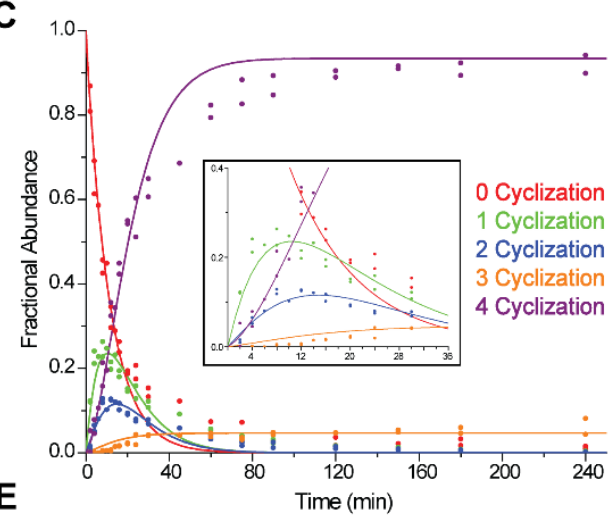

E

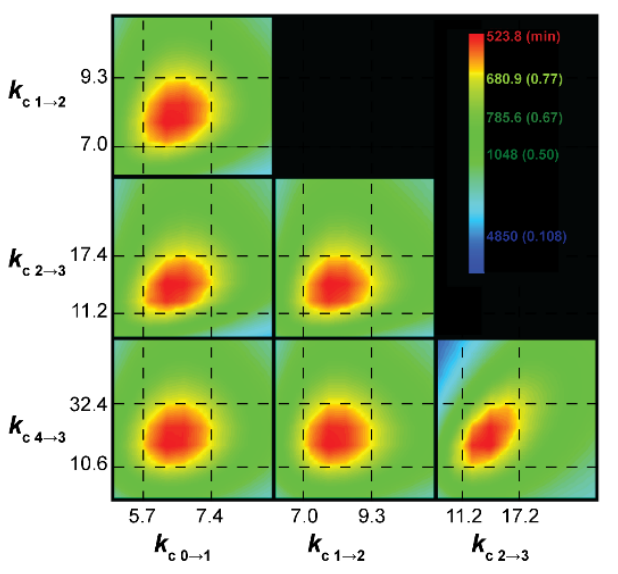

B

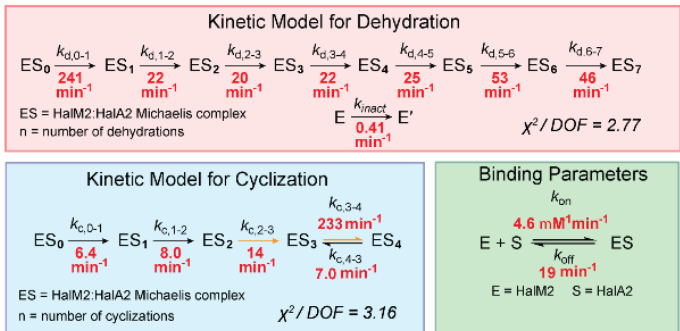
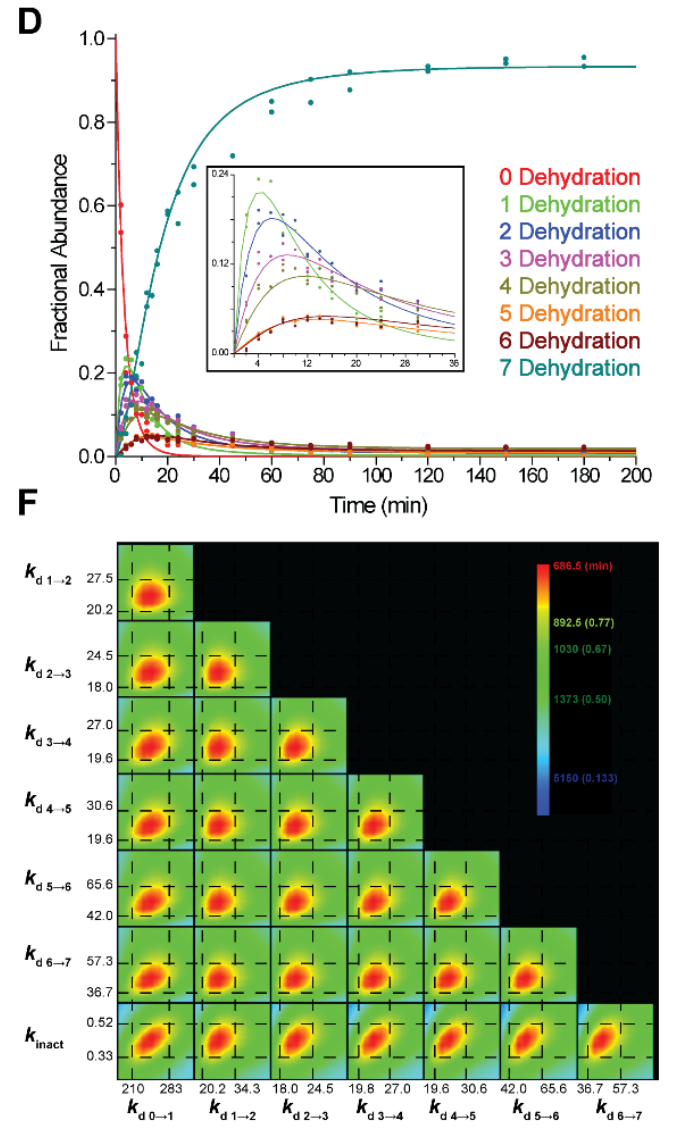

Figure S7. Simulated kinetic data for HalM2T386A/D387A. A) LC-ESI-MS spectra of the HalM2T386A/D387A/HalA2 reaction mixture shown as a function of reaction time. The $8^{+}$ charge state is shown. The numbering above the peaks indicates the number of 
dehydrated Ser/Thr residues in the intermediate. Peptides have been NEM-alkylated as described in the Supporting Methods to reveal the number of thioether rings. B) Kinetic models and binding parameters used for the simulation of dehydration and cyclization kinetic data (see also Figures S3-S4). Colored arrows represent apparent rate constants that were linked during the simulation. The goodness of fit is indicated by the $\chi^{2} / D O F$ statistic. Kinetic data for cyclization (panel C) and dehydration (panel D) simulated with the models in (B). The fractional abundances of species containing the same number of dehydrations/cyclizations were summed prior to simulation as described in the supporting methods. The HalM2T386/D387A data could be simulated using the same mechanisms that were used for wt HalM2, with the exception that a slow enzyme inactivation step was included in the dehydration mechanism $\left(k_{\text {inact }}=0.41 \mathrm{~min}^{-1}\right) . \mathrm{E}$ and $\mathrm{F}$ ) FitSpace calculations for the cyclization and dehydration data, respectively, were used to estimate variable kinetic parameter boundaries at a threshold of 1.2 times the $\chi^{2}$ minimum of the global fit (the red portion of each individual sub-plot). Rate constants are in units of min $^{-}$ 1. All fitted parameter values and their corresponding FitSpace boundaries are reported in Table 1 of the main text. 
A

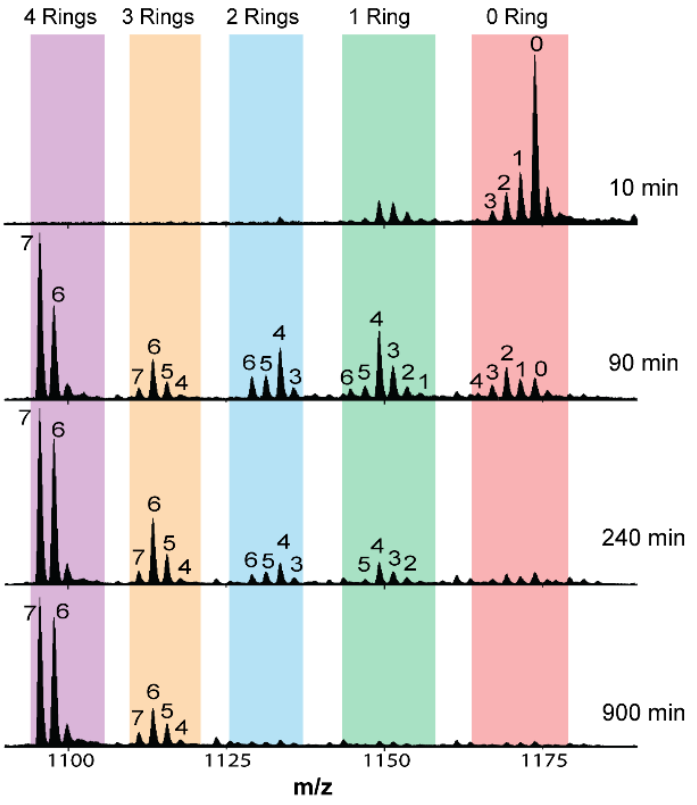

C

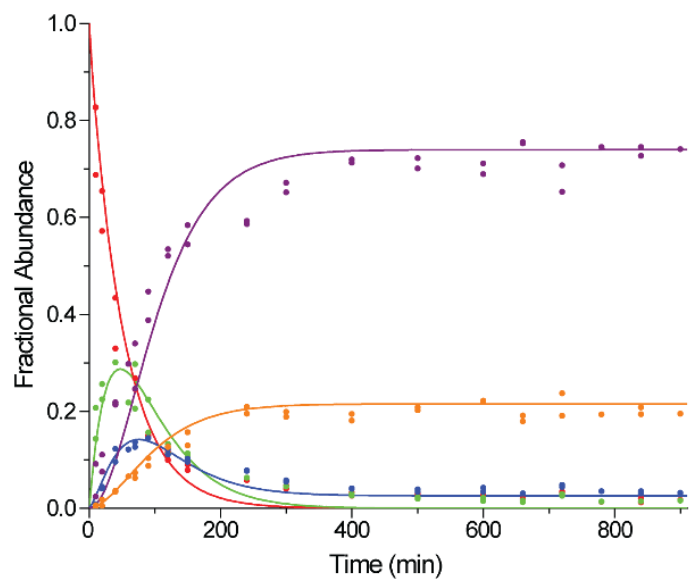

E

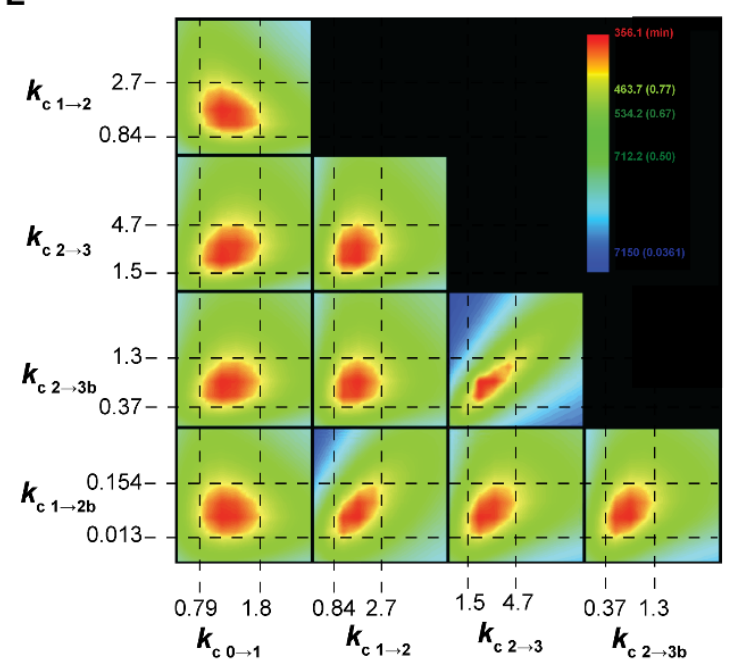

B

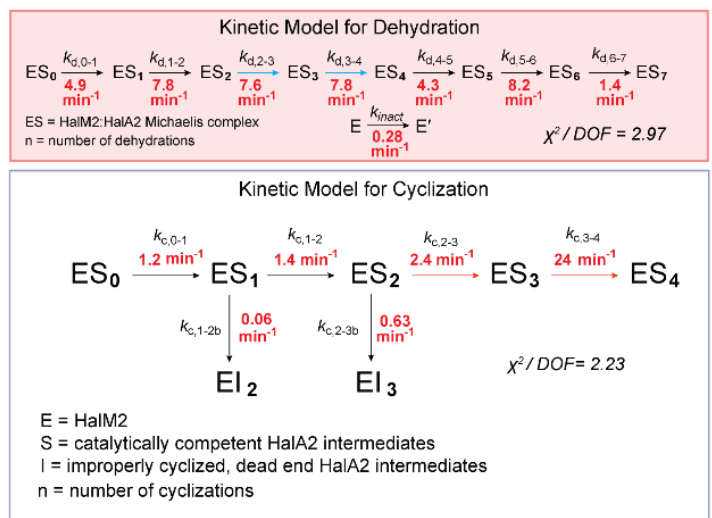

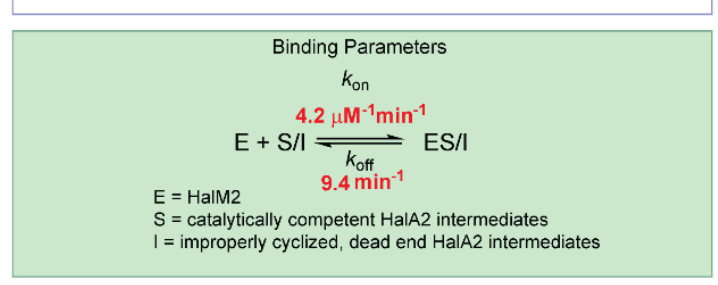

D

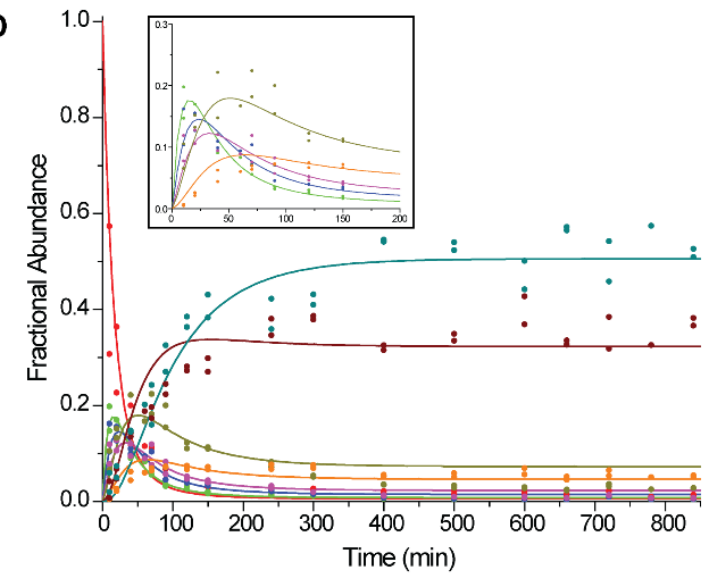

F

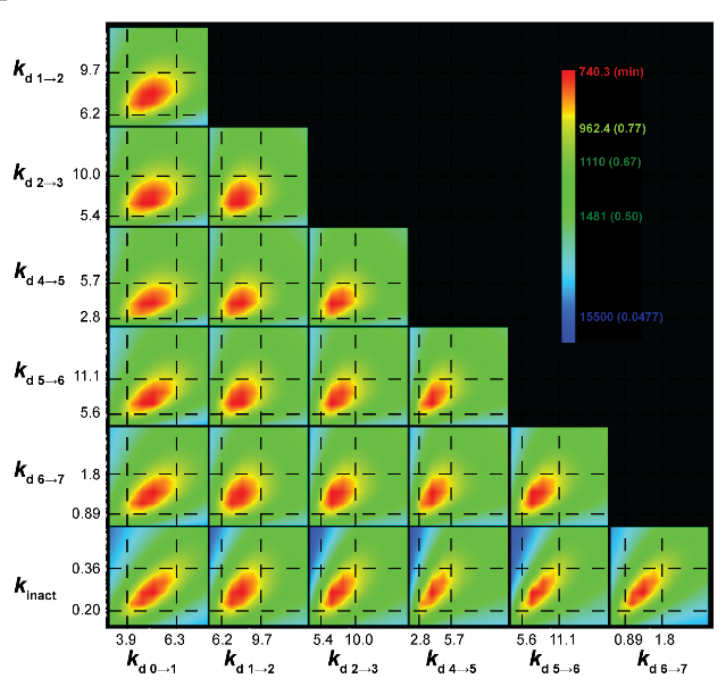


Figure S8. Simulated kinetic data for HalM2Р349A. A) LC-ESI-MS spectra of the HalM2P349A/HalA2 reaction mixture shown as a function of reaction time. The $8^{+}$charge state is shown. The numbering above the peaks indicates the number of dehydrated Ser/Thr residues in the intermediate. Peptides have been NEM-alkylated as described in the Supporting Methods to reveal the number of thioether rings. B) Kinetic models and binding parameters used for the simulation of dehydration and cyclization kinetic data (see also Figures S3-S4). Colored arrows represent apparent rate constants that were linked during the simulation. The goodness of fit is indicated by the $\chi^{2} / D O F$ statistic. Kinetic data for cyclization (panel C) and dehydration (panel D) simulated with the models in (B). The fractional abundances of species containing the same number of dehydrations/cyclizations were summed prior to simulation as described in the supporting methods. Relative to wt HalM2, the HalM2 ${ }_{\mathrm{P} 349 \mathrm{~A}}$ data could be simulated using the same mechanisms that were used for wt HaIM2, with the exception that the $3^{\text {rd }}$ cyclization step was also made reversible, and that a slow enzyme inactivation step was included in the dehydration mechanism ( $k_{\text {inact }}=0.28 \mathrm{~min}^{-1}$ ). $\mathrm{E}$ and F) FitSpace calculations for the cyclization and dehydration data, respectively, were used to estimate variable kinetic parameter boundaries at a threshold of 1.2 times the $\chi^{2}$ minimum of the global fit (the red portion of each individual sub-plot). Rate constants are in units of $\mathrm{min}^{-1}$. All fitted parameter values and their corresponding FitSpace boundaries are reported in Table 1 of the main text. 


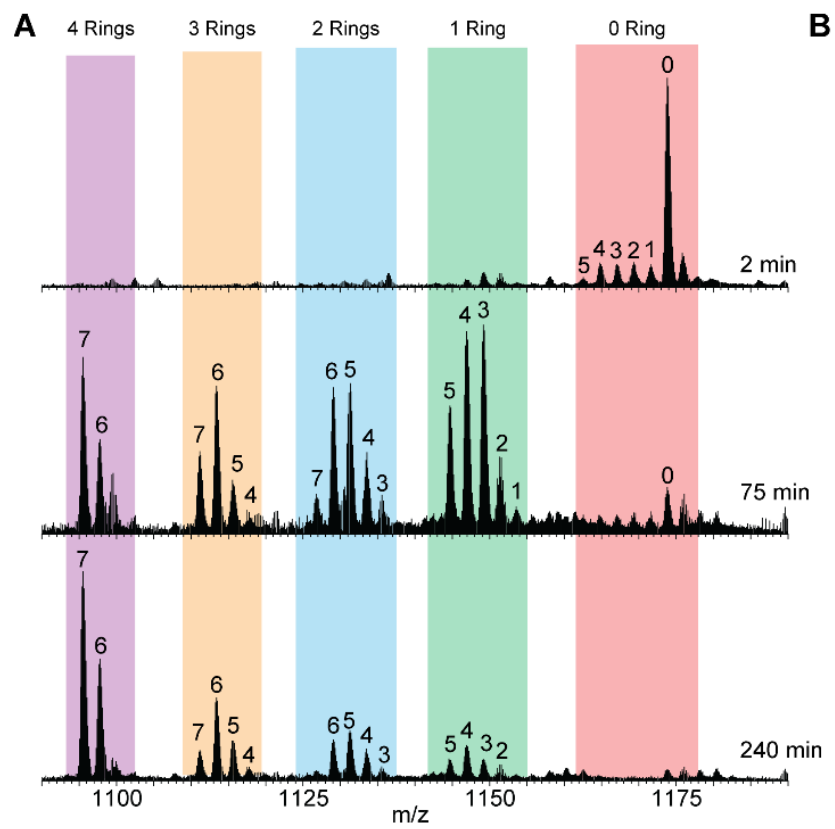

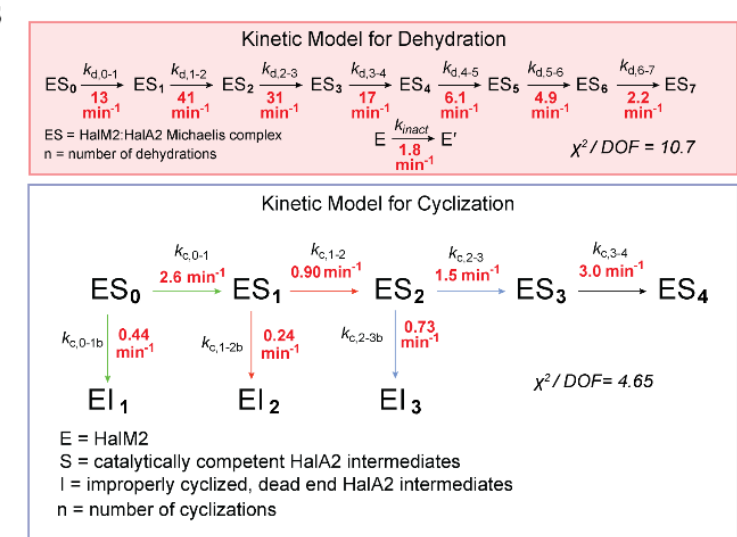
$\mathrm{n}=$ number of cyclizations
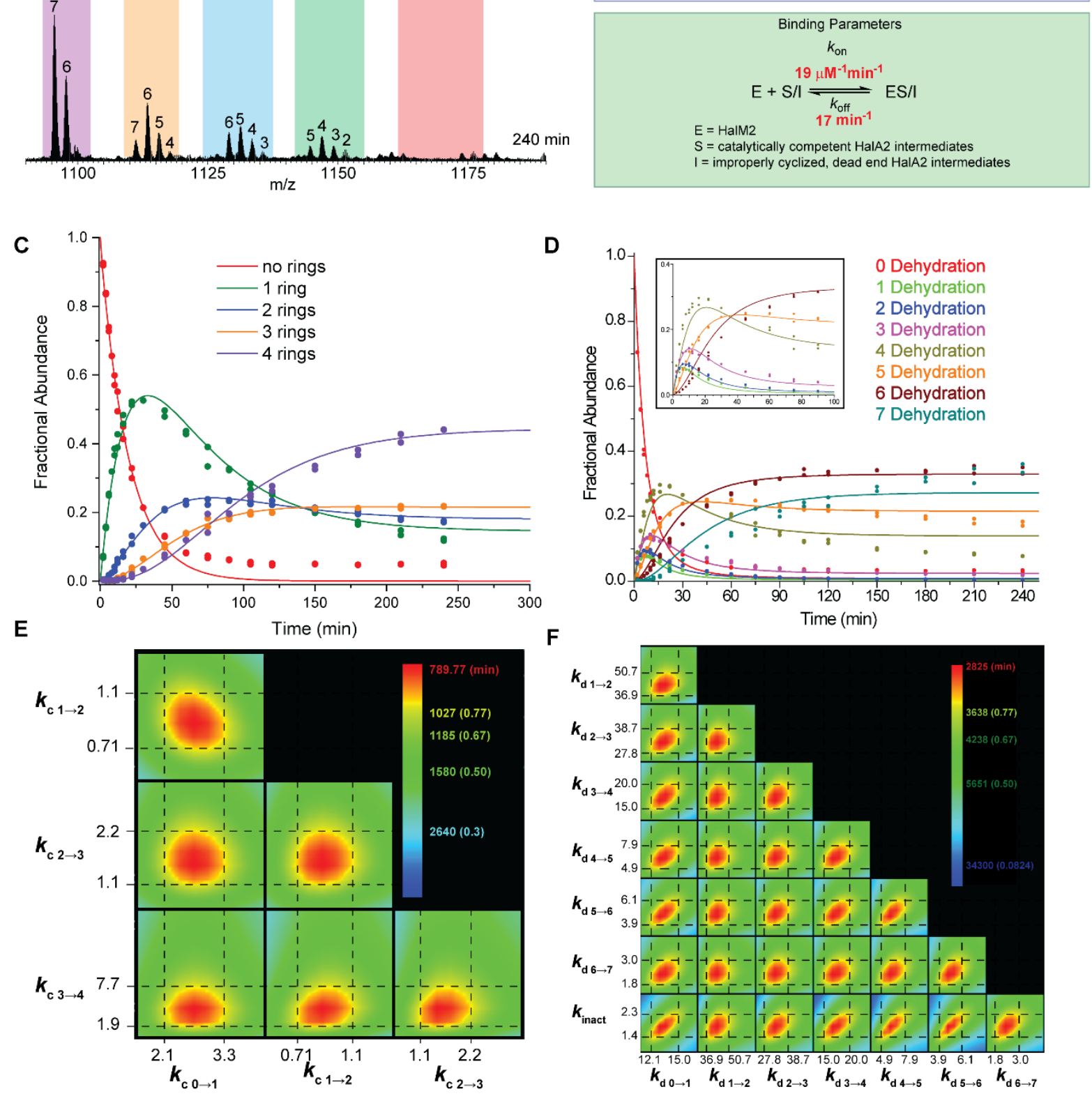
Figure S9. Simulated kinetic data for HalM2GS635-644. A) LC-ESI-MS spectra of the HalM2GS635-644/HalA2 reaction mixture shown as a function of reaction time. The $8^{+}$charge state is shown. The numbering above the peaks indicates the number of dehydrated Ser/Thr residues in the intermediate. Peptides have been NEM-alkylated as described in the Supporting Methods to reveal the number of thioether rings. B) Kinetic models and binding parameters used for the simulation of dehydration and cyclization kinetic data (see also Figures S3-S4). Colored arrows represent apparent rate constants that were linked during the simulation. The goodness of fit is indicated by the $\chi^{2} / D O F$ statistic. Kinetic data for cyclization (panel C) and dehydration (panel D) simulated with the models in (B). The fractional abundances of species containing the same number of dehydrations/cyclizations were summed prior to simulation as described in the supporting methods. Relative to wt HalM2, the cyclization model was altered to include the formation of dead-end species as described in the main text and supporting discussion. A slow enzyme inactivation step was again necessary in the dehydration mechanism $\left(k_{\text {inact }}=\right.$ $1.8 \mathrm{~min}^{-1}$ ) to achieve a good fit. E and F) FitSpace calculations for the cyclization and dehydration data, respectively, were used to estimate variable kinetic parameter boundaries at a threshold of 1.2 times the $\chi^{2}$ minimum of the global fit (the red portion of each individual sub-plot). Rate constants are in units of $\mathrm{min}^{-1}$. All fitted parameter values and their corresponding FitSpace boundaries are reported in Table 1 of the main text. 


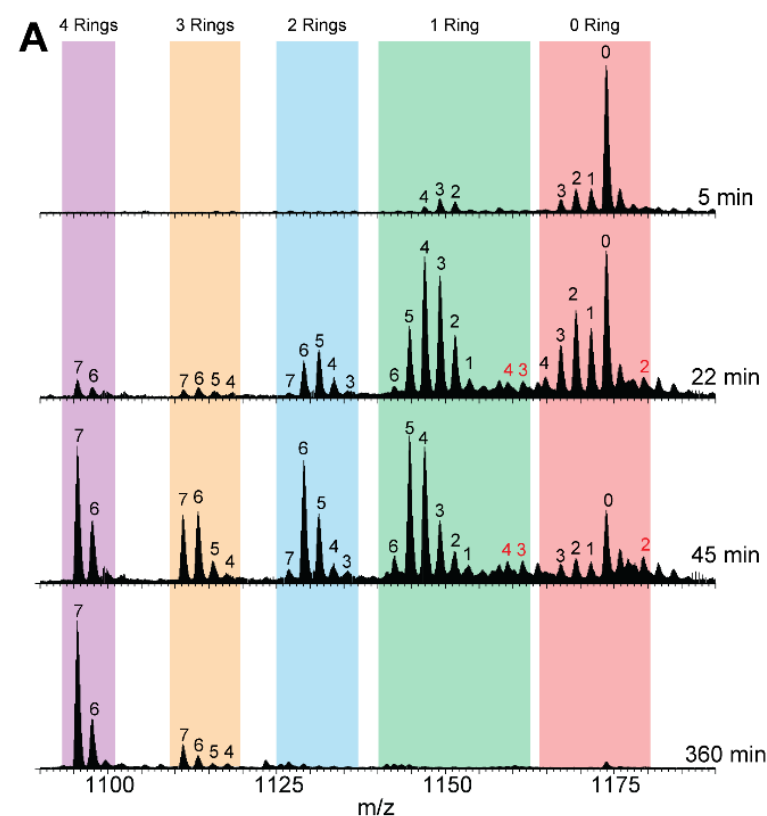

B
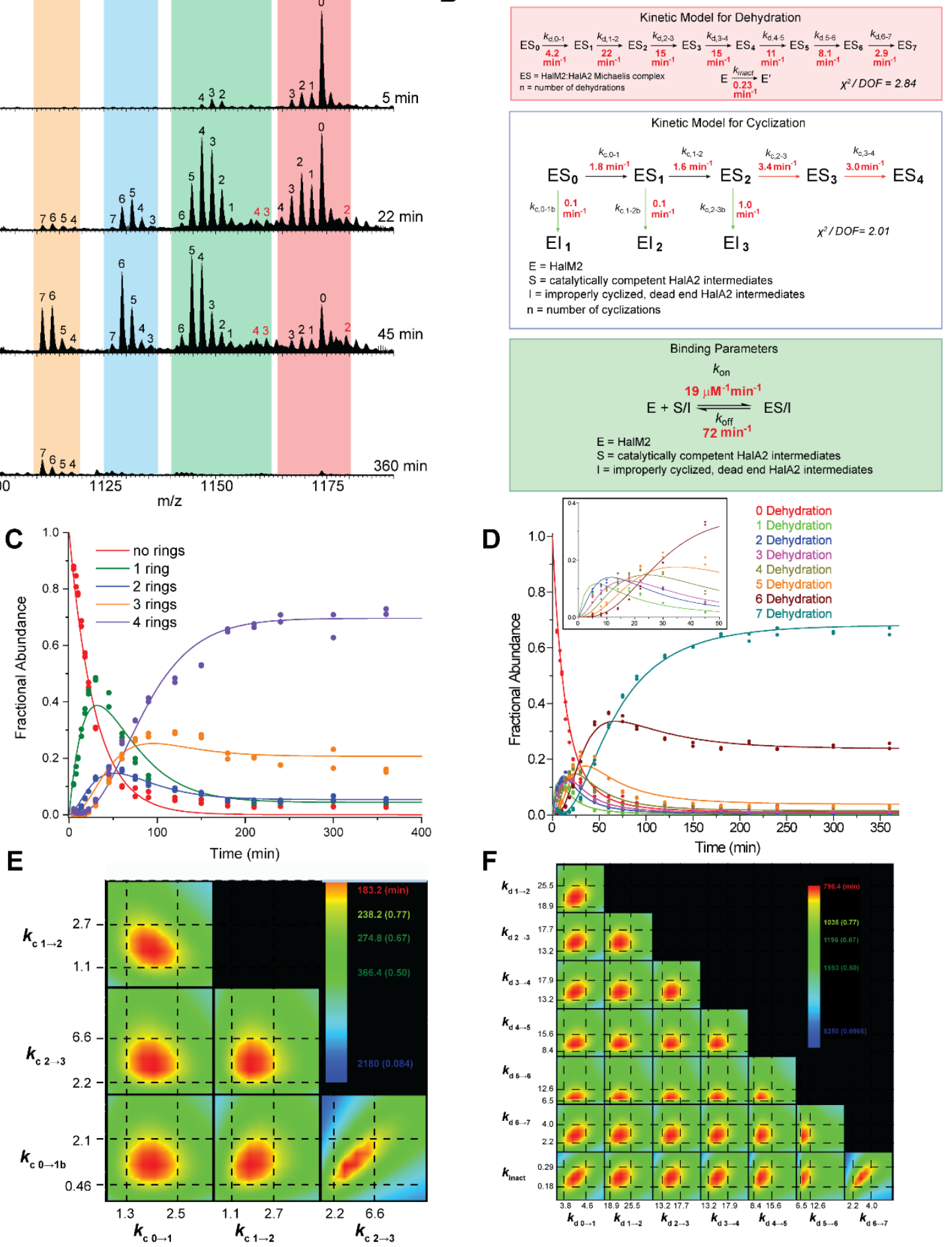

$\mathbf{F}$

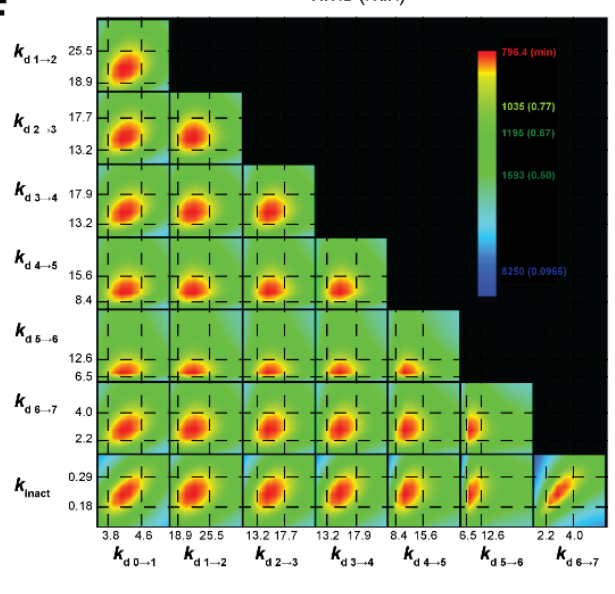

Figure S10. Simulated kinetic data for HalM2GS491-497. A) LC-ESI-MS spectra of the HalM2Gs491-497/HalA2 reaction mixture shown as a function of reaction time. The $8^{+}$charge state is shown. The numbering above the peaks indicates the number of dehydrated 
Ser/Thr residues in the intermediate. Peptides have been NEM-alkylated as described in the Supporting Methods to reveal the number of thioether rings. B) Kinetic models and binding parameters used for the simulation of dehydration and cyclization kinetic data (see also Figures S3-S4). Colored arrows represent apparent rate constants that were linked during the simulation. The goodness of fit is indicated by the $\chi^{2} / D O F$ statistic. Kinetic data for cyclization (panel C) and dehydration (panel D) simulated with the models in (B). The fractional abundances of species containing the same number of dehydrations/cyclizations were summed prior to simulation as described in the supporting methods. Relative to wt HalM2, the cyclization model was altered to include the formation of dead-end species as described in the main text and supporting discussion. A slow enzyme inactivation step was necessary in the dehydration mechanism ( $k_{\text {inact }}=$ $0.23 \mathrm{~min}^{-1}$ ) to achieve a good fit. E and F) FitSpace calculations for the cyclization and dehydration data, respectively, were used to estimate variable kinetic parameter boundaries at a threshold of 1.2 times the $\chi^{2}$ minimum of the global fit (the red portion of each individual sub-plot). Rate constants are in units of $\mathrm{min}^{-1}$. All fitted parameter values and their corresponding FitSpace boundaries are reported in Table 1 of the main text. 
A

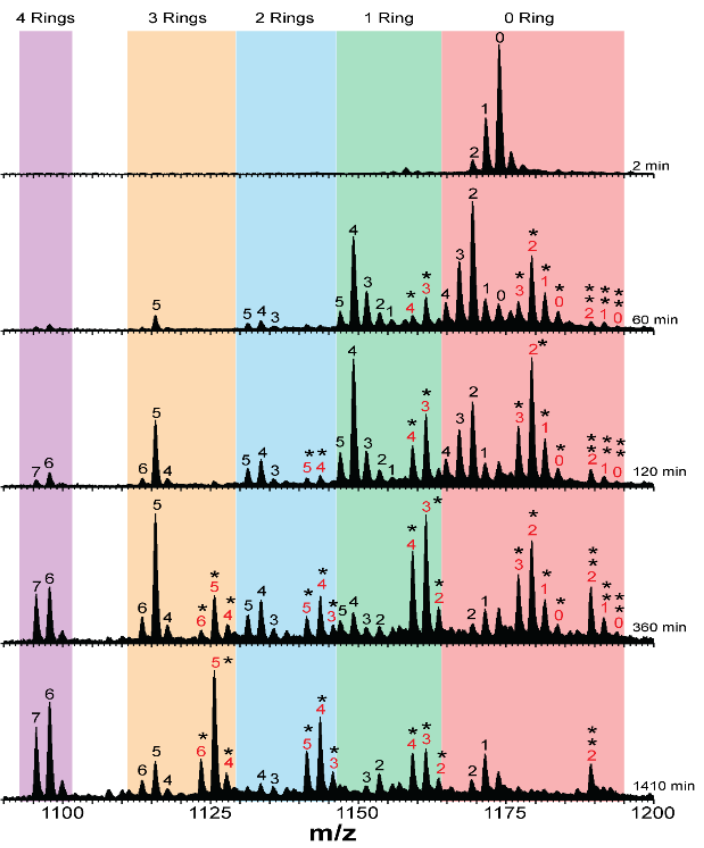

C

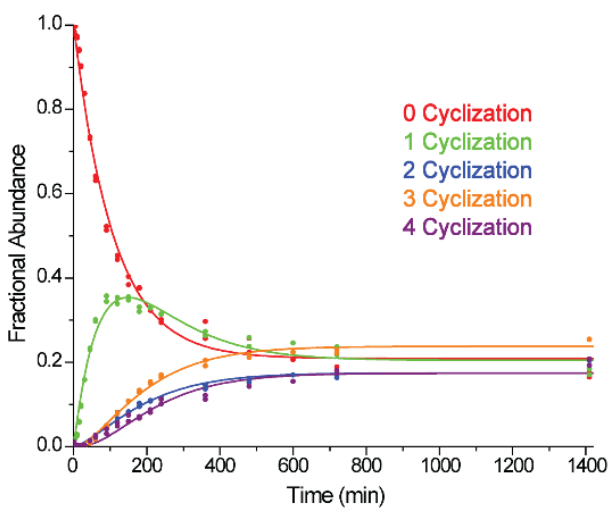

E

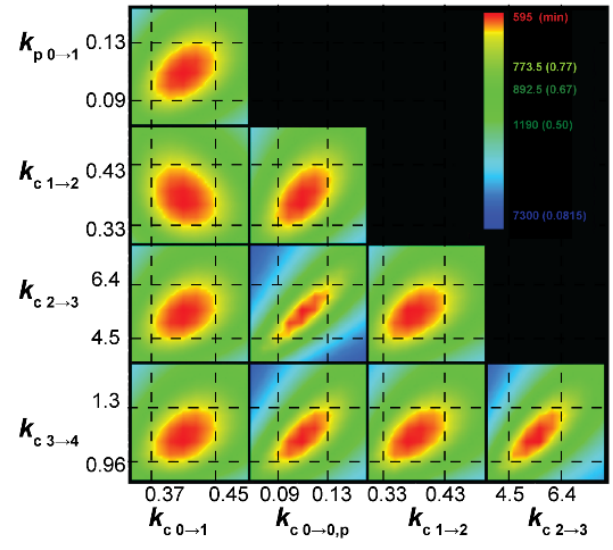

B

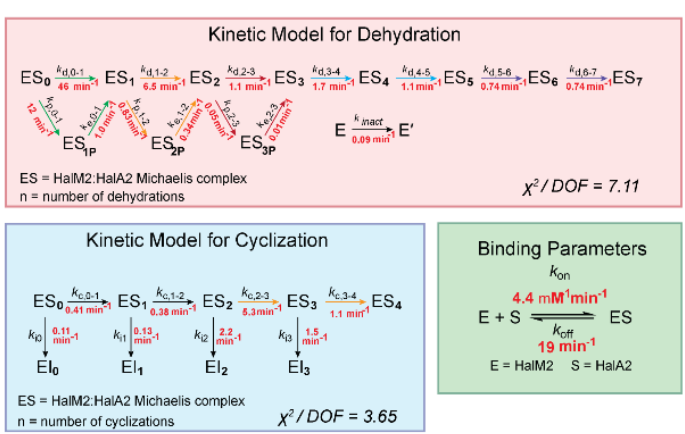

D

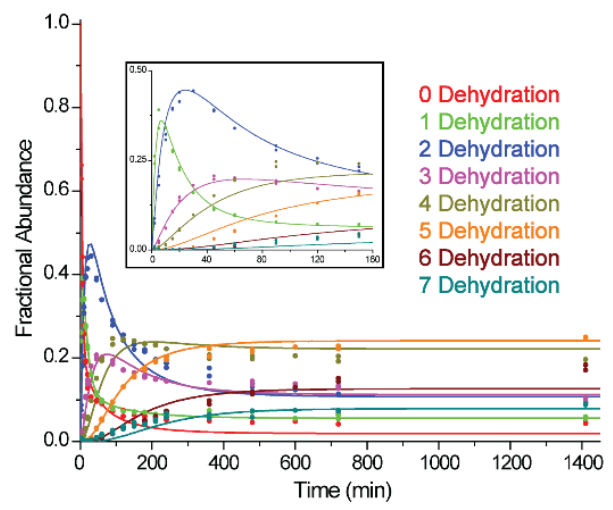

$\mathbf{F}$

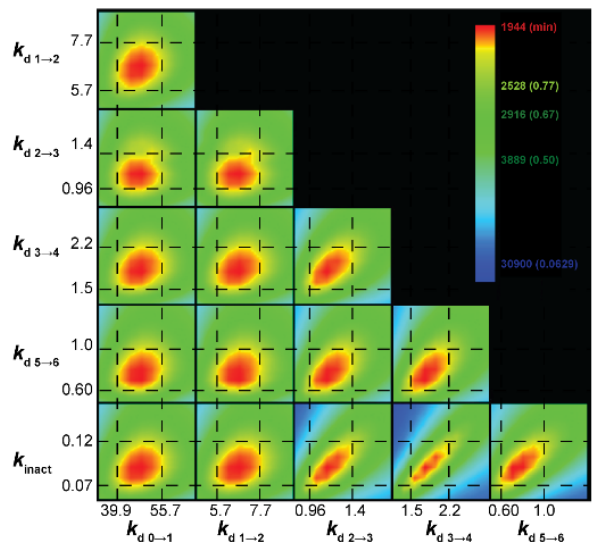

Figure S11. Simulated kinetic data for HalM2GS389-396. A) LC-ESI-MS spectra of the HalM2Gs389-396/HalA2 reaction mixture shown as a function of reaction time. The $8^{+}$charge 
state is shown. The numbering above the peaks indicates the number of dehydrated Ser/Thr residues in the intermediate. Peptides have been NEM-alkylated as described in the Supporting Methods to reveal the number of thioether rings. B) Kinetic models and binding parameters used for the simulation of dehydration and cyclization kinetic data (see also Figures S3-S4). Colored arrows represent apparent rate constants that were linked during the simulation. The goodness of fit is indicated by the $\chi^{2} / D O F$ statistic. Kinetic data for cyclization (panel $C$ ) and dehydration (panel D) simulated with the models in (B). The fractional abundances of species containing the same number of dehydrations/cyclizations were summed prior to simulation as described in the supporting methods. The phosphorylated species were modeled as dead-end side products in the cyclization mechanism, and as parallel reaction pathways in the dehydration reaction with a slow phosphate elimination step. See the main text and supplemental discussion for additional details. A slow enzyme inactivation step was necessary in the dehydration mechanism ( $k_{\text {inact }}=0.23 \mathrm{~min}^{-1}$ ) to achieve a good fit. $\mathrm{E}$ and $\mathrm{F}$ ) FitSpace calculations for the cyclization and dehydration data, respectively, were used to estimate variable kinetic parameter boundaries at a threshold of 1.2 times the $\chi^{2}$ minimum of the global fit (the red portion of each individual sub-plot). Rate constants are in units of $\mathrm{min}^{-1}$. All fitted parameter values and their corresponding FitSpace boundaries are reported in Table 1 of the main text. 

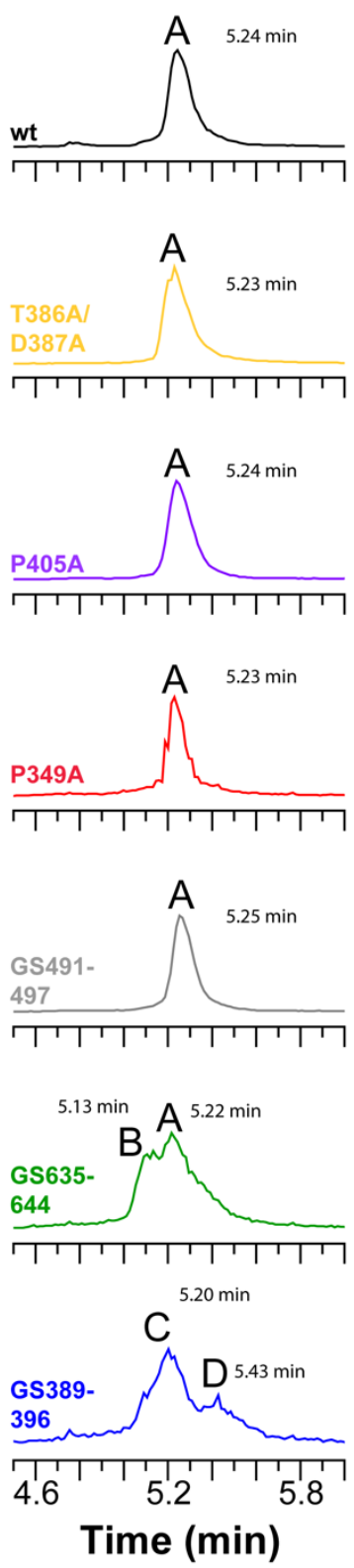
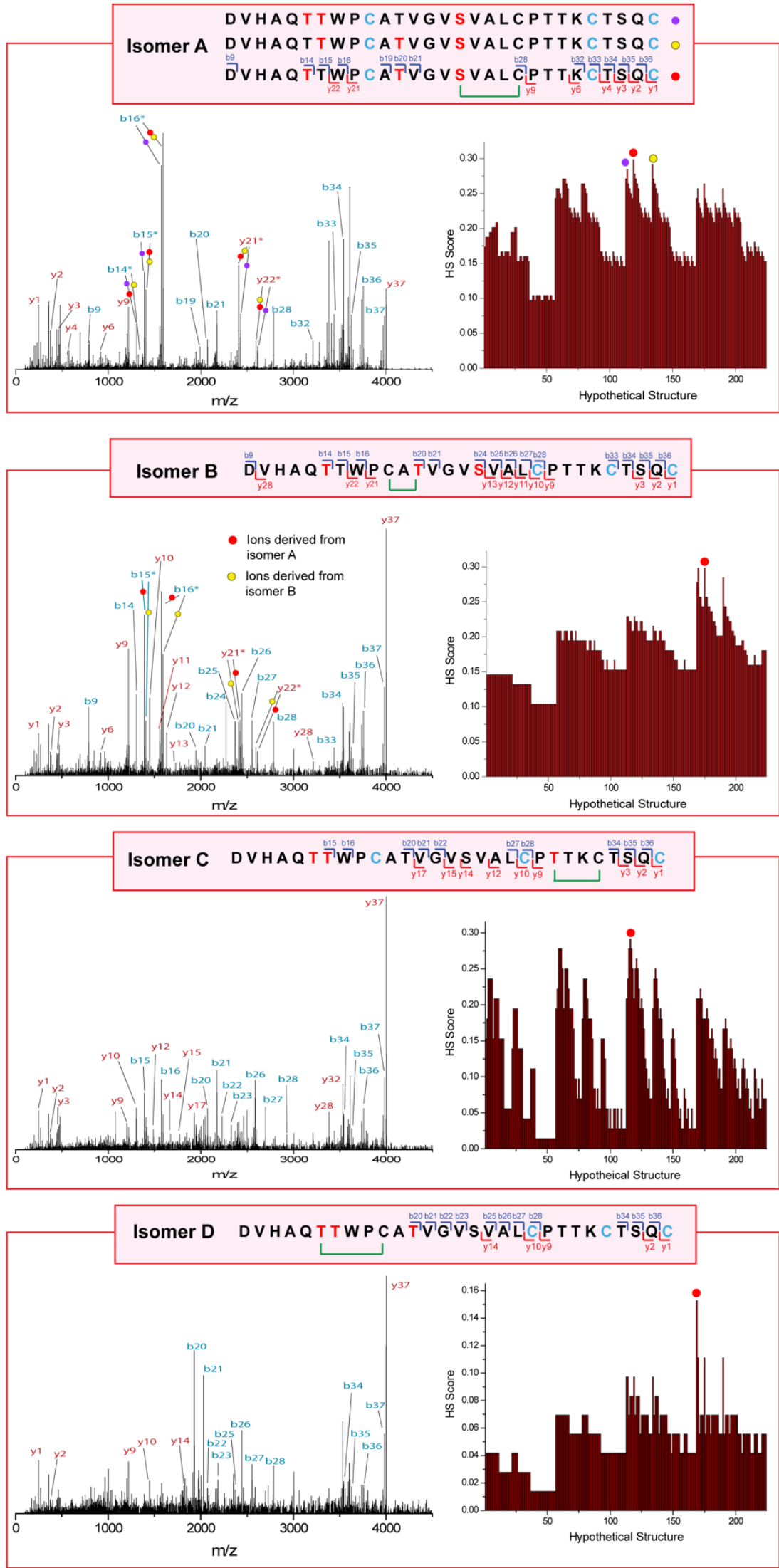
Figure S12. Retention time analysis and tandem mass spectrometry for GluC-digested HalA2 species containing one thioether ring and two additional dehydrations, [1L2D]. The $4^{+}$ion of [1L2D] $(m / z=1001.21 D a)$ was chosen for fragmentation by collision induced dissociation. Extracted ion chromatograms (EICs) for [1L2D] produced in the reactions of each HalM2 enzyme are shown on the left, with the various cyclization isomers labeled A-D. On the right are shown annotated tandem mass spectra for each cyclization isomer along with HSEE predictions. The highest scoring structure in the HSEE analysis is indicated with a red dot, and a model of this structure is shown above the data for each isomer. In the model, the dehydrated Ser/Thr resides are colored red and NEM-alkylated Cys residues are colored blue. The proposed thioether ring topology and $b$ and $y$ fragment ions observed in the tandem mass spectrum are indicated. In the tandem mass spectrum of isomer A (shown for the wt HalM2), a series of dehydrated fragment ions (indicated with *) not matching the most likely hypothetical structure were seen. These represent fragment ions from other dehydration isomers (indicated with the purple and gold dots) and suggest that the dehydration order is not strict. The spectrum for isomer $B$ derived from the HalM2GS635-644 reaction contains ions from the closely eluting isomer A (indicated with red dots). Isomer B appears to have a non-native ring A between Cys 5 and Thr7, as indicated by the $b 14-16$ and $y 20-21$ fragment ions, and by the $b 20-27$ ions which lack NEM alkylation and suggest that Cys 5 is engaged in a thioether ring. Isomers $C$ and $D$ produced in the HalM2GS389-396 reaction appear to contain rings $C$ and A, respectively, as indicated. 

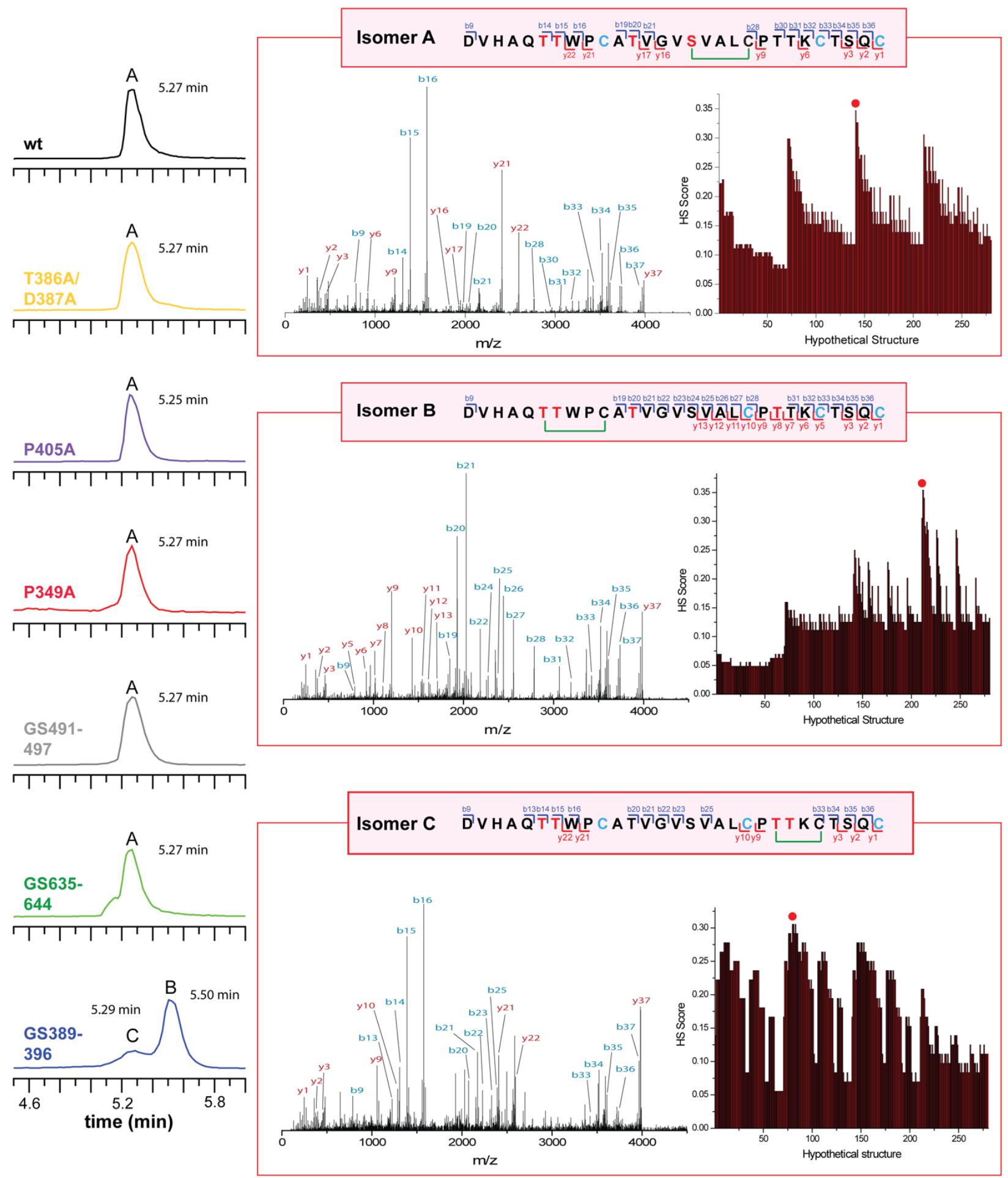

Figure S13. Retention time analysis and tandem mass spectrometry for GluC-digested HalA2 species containing one thioether ring and three additional dehydrations, [1L3D]. The $4^{+}$ion of [1L3D] was chosen for fragmentation $(\mathrm{m} / z=996.705 \mathrm{Da})$. Extracted ion chromatograms (EICs) for [1L3D] produced in the reactions of each HalM2 enzyme are 
shown on the left, with the various cyclization isomers labeled A-C. On the right are shown annotated tandem mass spectra for each cyclization isomer along with HSEE predictions. The highest scoring structure in the HSEE analysis is indicated with a red dot, and a model of this structure is shown above the data for each isomer. In the model, the dehydrated Ser/Thr resides are colored red and NEM-alkylated Cys residues are colored blue. The proposed thioether ring topology is indicated, along with the $b$ and $y$ fragment ions observed in the tandem mass spectrum. Most enzymes produced a single cyclization isomer containing ring B (Ser11-Cys15). The HalM2GS389-396 variant again produced a pair of isomers containing either ring $\mathrm{A}$ or ring $\mathrm{C}$. 


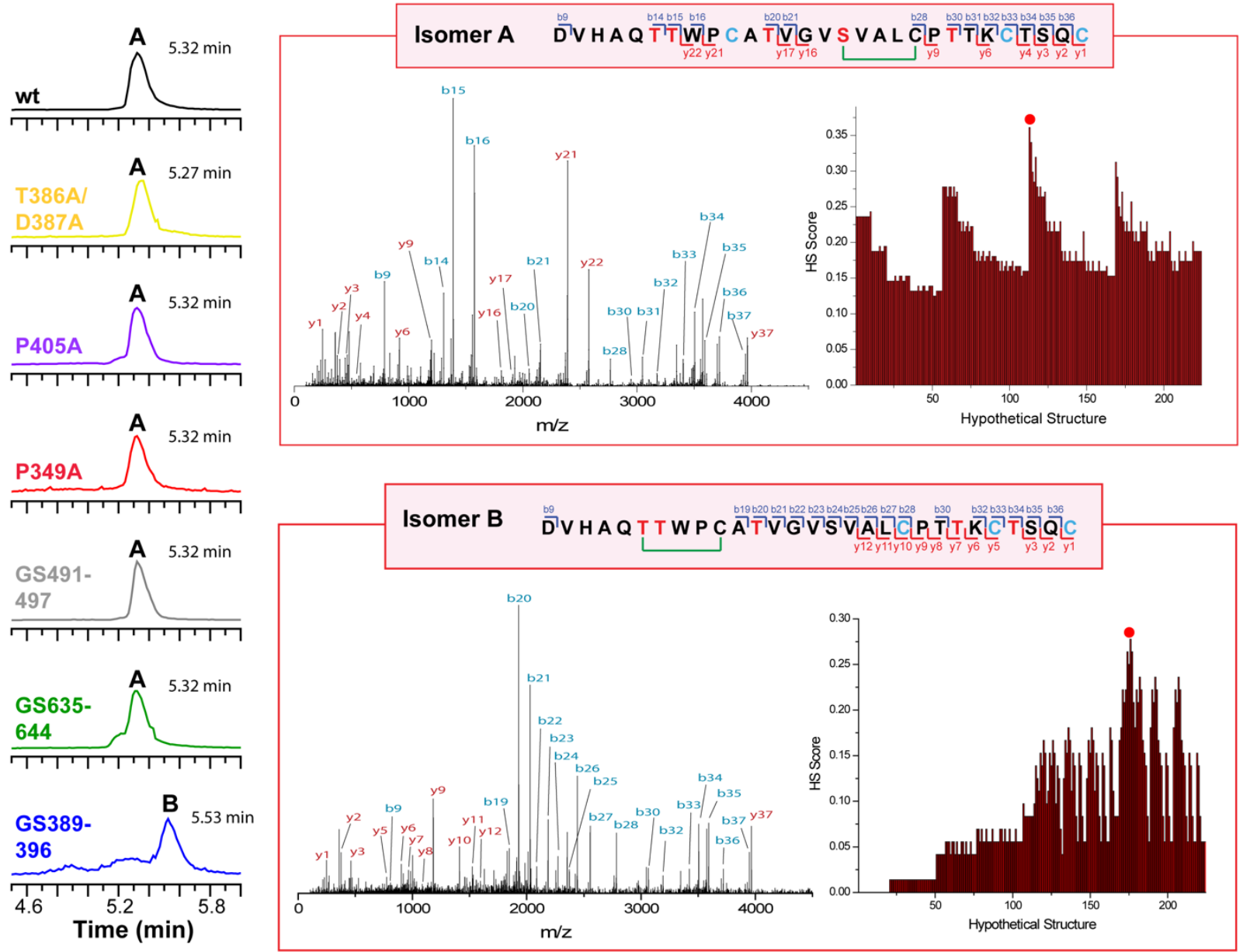

Figure S14. Retention time analysis and tandem mass spectrometry for GluC-digested HalA2 species containing one thioether ring and four additional dehydrations, [1L4D]. The $4^{+}$ion of [1L4D] was chosen for fragmentation $(\mathrm{m} / z=992.202 \mathrm{Da})$. Extracted ion chromatograms (EICs) for [1L4D] produced in the reactions of each HalM2 enzyme are shown on the left, with the various cyclization isomers labeled A-B. On the right are shown annotated tandem mass spectra for each cyclization isomer along with HSEE predictions. The highest scoring structure in the HSEE analysis is indicated with a red dot, and a model of this structure is shown above the data for each isomer. In the model, the dehydrated Ser/Thr resides are colored red and NEM-alkylated Cys residues are colored blue. The proposed thioether ring topology is indicated, along with the $b$ and $y$ fragment ions that were observed in the tandem mass spectrum. Most enzymes produced a single cyclization isomer containing ring B (Ser11-Cys15). The HalM2GS389-396 variant generated an isomer containing ring $A($ Thr1-Cys5). 


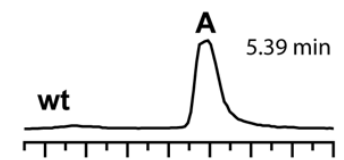

A
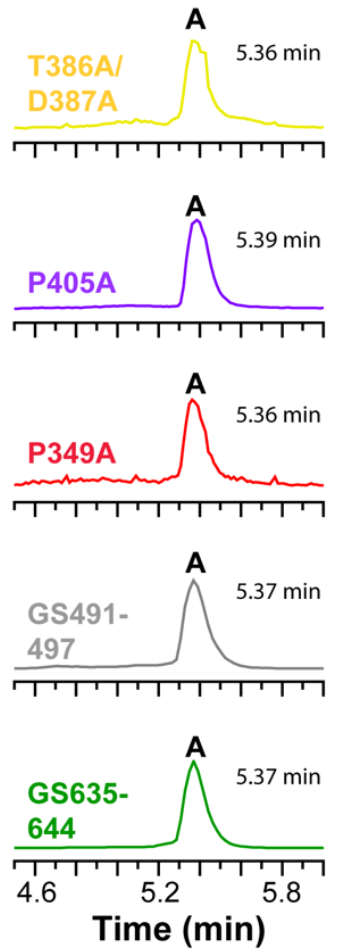

Figure S15. Retention time analysis and tandem mass spectrometry for GluC-digested HalA2 species containing one thioether ring and five additional dehydrations, [1L5D]. The $4^{+}$ion of [1L5D] was chosen for fragmentation $(\mathrm{m} / \mathrm{z}=992.202 \mathrm{Da})$. Extracted ion chromatograms (EICs) for [1L5D] produced in the reactions of each HalM2 enzyme are shown on the left. On the right is shown the annotated tandem mass spectrum of isomer A derived from wt HalM2, along with the corresponding HSEE prediction. The highest scoring structure in the HSEE analysis is indicated with a red dot, and a model of this structure is shown above the data for the isomer. In the model, the dehydrated Ser/Thr resides are colored red and NEM-alkylated Cys residues are colored blue. The proposed thioether ring topology is indicated, along with the $b$ and $y$ fragment ions observed in the tandem mass spectrum. 

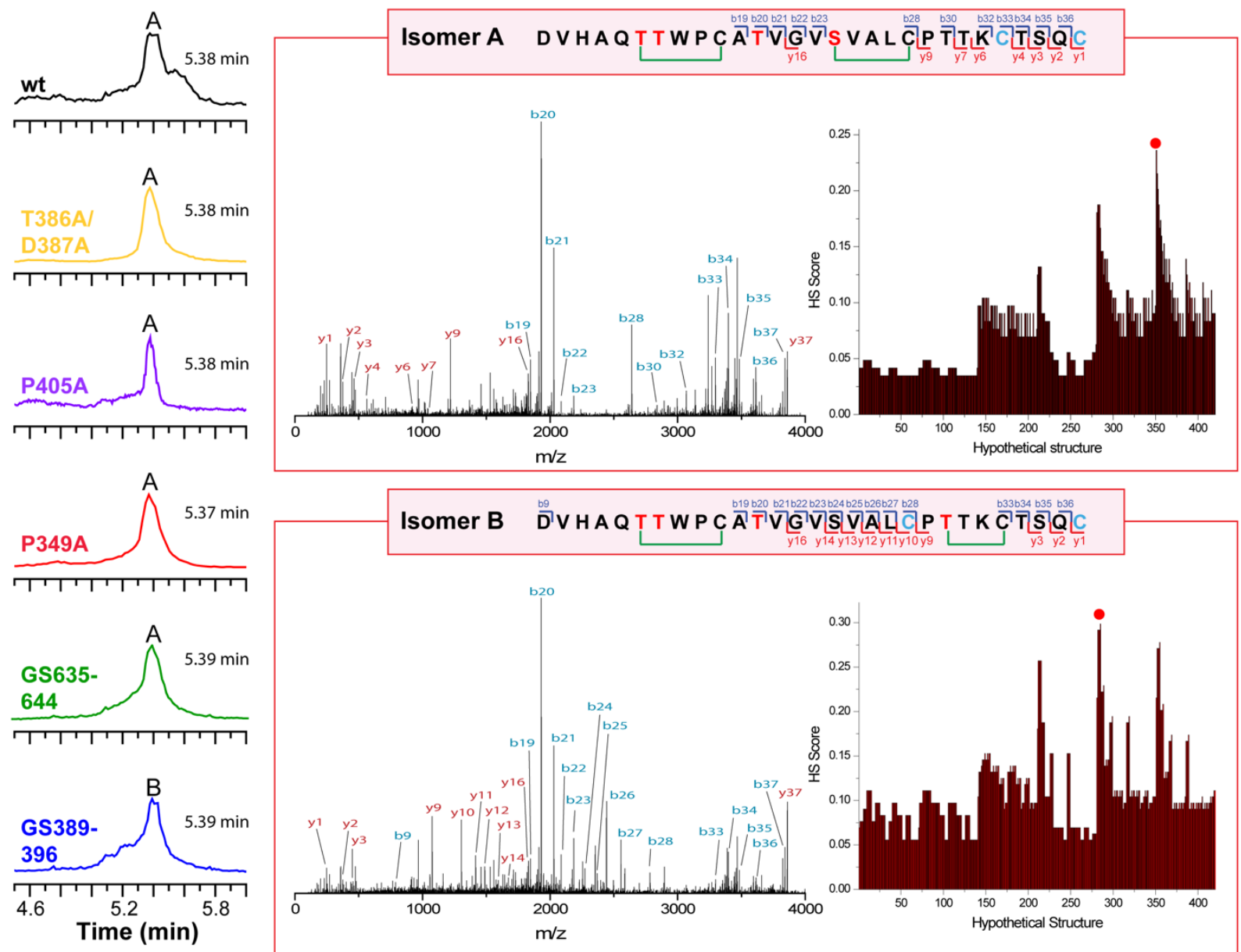

Figure S16. Retention time analysis and tandem mass spectrometry for GluC-digested HalA2 species containing two thioether rings and two additional dehydrations, [2L2D]. The $4^{+}$ion of [2L2D] was chosen for fragmentation $(\mathrm{m} / z=965.443 \mathrm{Da})$. Extracted ion chromatograms (EICs) for [2L2D] produced in the reactions of each HalM2 enzyme are shown on the left, with the various cyclization isomers labeled A-B. On the right are shown annotated tandem mass spectra for each cyclization isomer along with HSEE predictions. The highest scoring structure in the HSEE analysis is indicated with a red dot, and a model of this structure is shown above the data for each isomer. In the model, the dehydrated Ser/Thr resides are colored red and NEM-alkylated Cys residues are colored blue. The proposed thioether ring topology is indicated, along with the $b$ and $y$ fragment ions observed in the tandem mass spectrum. The spectra for isomers $A$ and $B$ are derived from intermediates in the HalM2T386A/D387A and HalM2GS386-389 reactions, respectively. Most enzymes produced an isomer containing thioether rings $A$ and $B$, while HalM2Gs386- 
389 generated a species with rings $\mathrm{A}$ and $\mathrm{C}$. This latter topology is indicated by the $b_{24-27}$ and $y_{10-13}$ ions that were not observed in the fragmentation spectrum of isomer $\mathrm{A}$. 

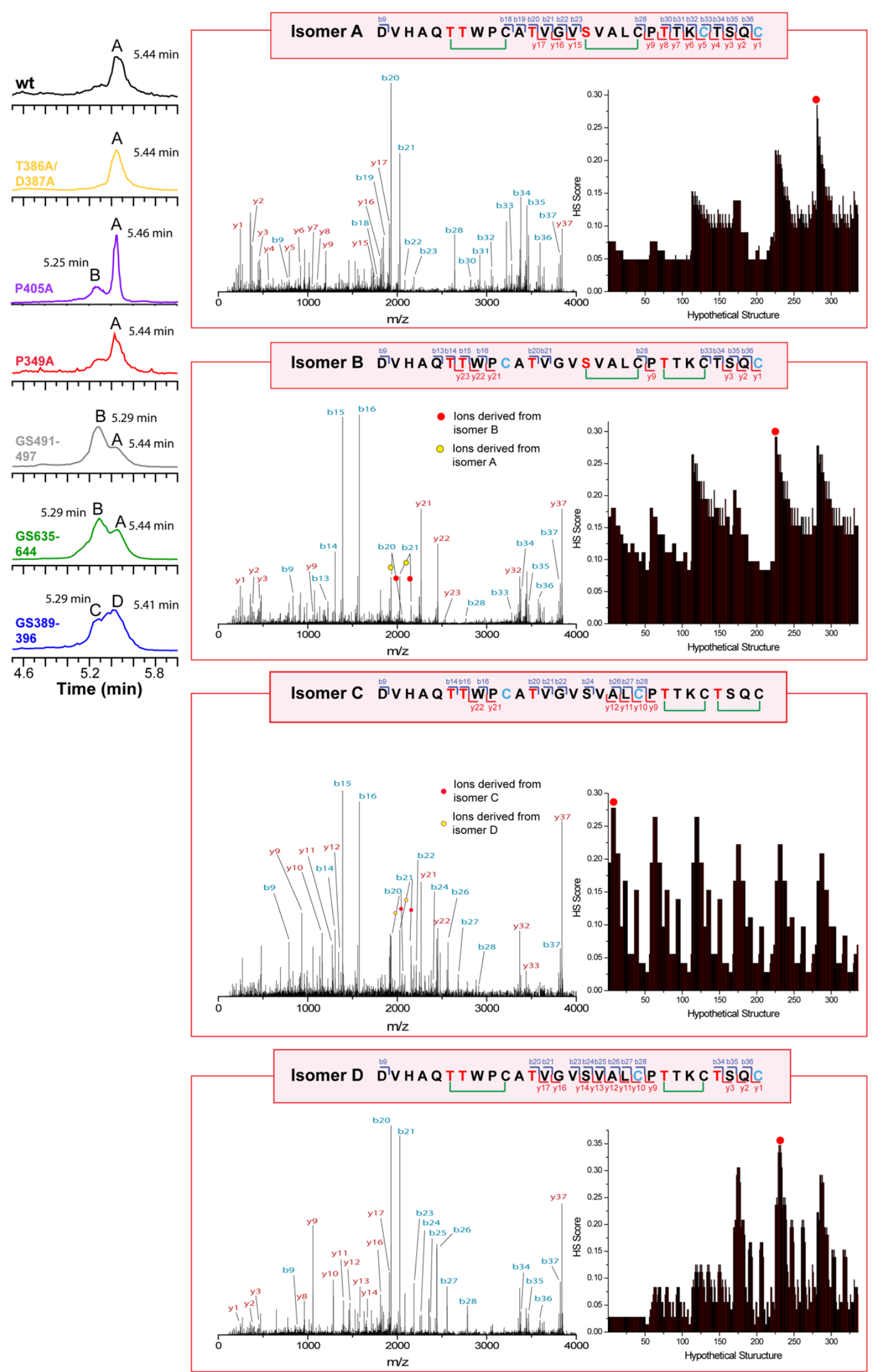
Figure S17. Retention time analysis and tandem mass spectrometry for GluC-digested HalA2 species containing two thioether rings and three additional dehydrations, [2L3D]. The $4^{+}$ion of [2L3D] was chosen for fragmentation $(\mathrm{m} / \mathrm{z}=960.941 \mathrm{Da})$. Extracted ion chromatograms (EICs) for [2L3D] produced in the reactions of each HalM2 enzyme are shown on the left, with the various cyclization isomers labeled A-D. On the right are shown annotated tandem mass spectra for each cyclization isomer along with HSEE predictions. The highest scoring structure in the HSEE analysis is indicated with a red dot, and a model of this structure is shown above the data for each isomer. In the model, the dehydrated Ser/Thr resides are colored red and NEM-alkylated Cys residues are colored blue. The proposed thioether ring topology is indicated, along with the $b$ and $y$ fragment ions observed in the tandem mass spectrum. The spectrum for isomer $A$ (shown for the HalM2T386A/D387A reaction) indicates the presence of thioether rings $A$ and $B$. The spectrum for isomer $\mathrm{B}$ (shown for the HalM2Gs491-497 reaction) shows strong $b_{14-16}$ and $y_{20-21}$ ions indicating the lack of ring $A$ and $a$ thioether topology containing rings $B$ and $C$. Note that the poor chromatographic separation of isomers leads to the appearance of fragment ions derived from isomer $A$. The spectra for isomers $C$ and $D$ are from the HalM2GS389-396 reaction. The fragmentation data for these isomers are consistent with thioether topologies containing rings $C-D$ and rings $A-C$, respectively. 


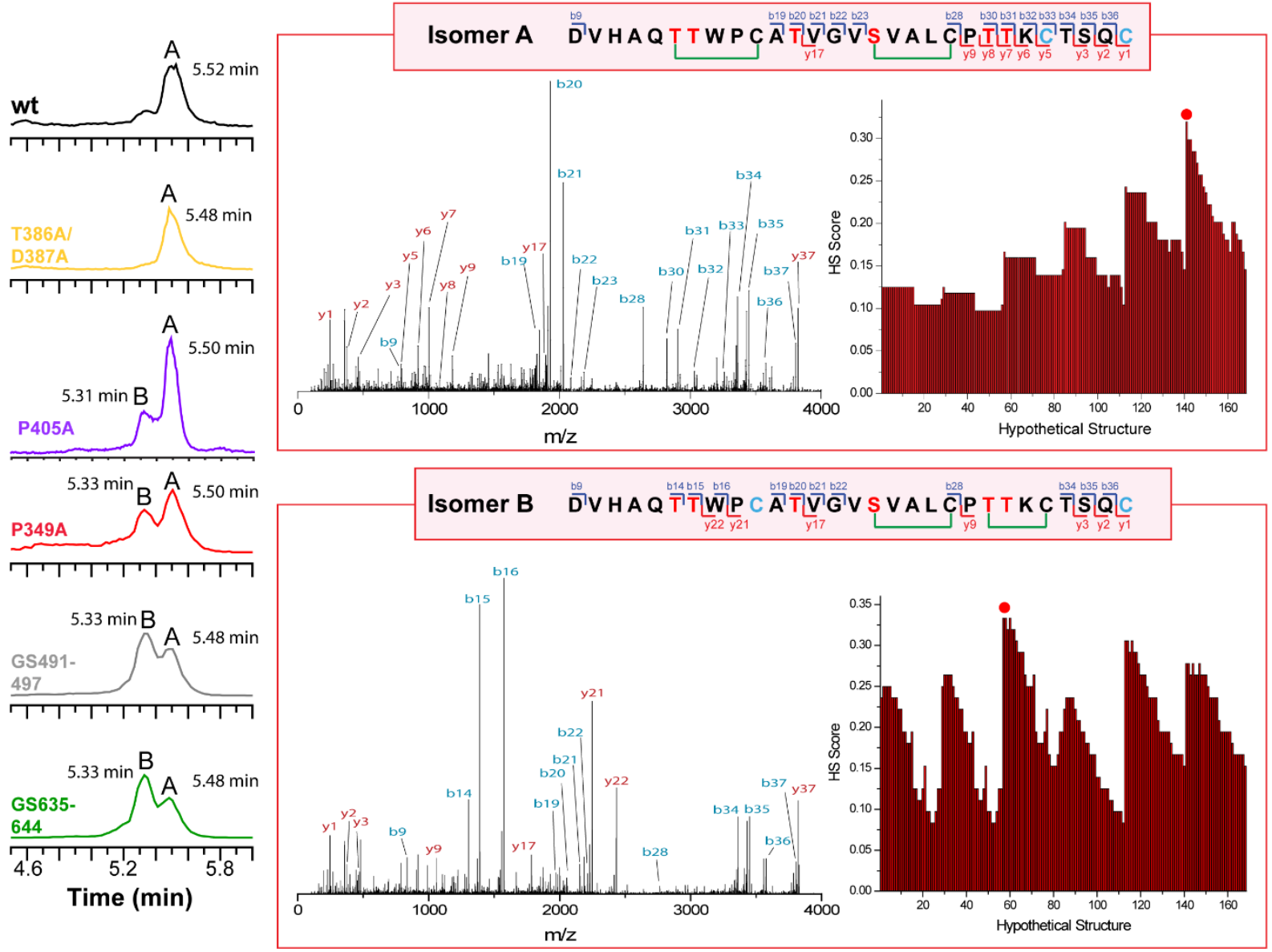

Figure S18. Retention time analysis and tandem mass spectrometry for GluC-digested HalA2 species containing two thioether rings and four additional dehydrations, [2L4D]. The $4^{+}$ion of [2L4D] was chosen for fragmentation $(\mathrm{m} / \mathrm{z}=956.438 \mathrm{Da})$. Extracted ion chromatograms (EICs) for [2L4D] produced in the reactions of each HalM2 enzyme are shown on the left, with the various cyclization isomers labeled A-B. On the right are shown annotated tandem mass spectra for each cyclization isomer along with HSEE predictions. The highest scoring structure in the HSEE analysis is indicated with a red dot, and a model of this structure is shown above the data for each isomer. In the model, the dehydrated Ser/Thr resides are colored red and NEM-alkylated Cys residues are colored blue. The thioether ring topology and $b$ and $y$ fragment ions observed in the tandem mass spectrum are indicated. The spectrum of isomer A (taken from the wt HalM2 reaction) again suggests a topology containing rings $A-B$, while isomer $B$ (spectrum taken from HalM2Gs635-644 contains rings B-C. 


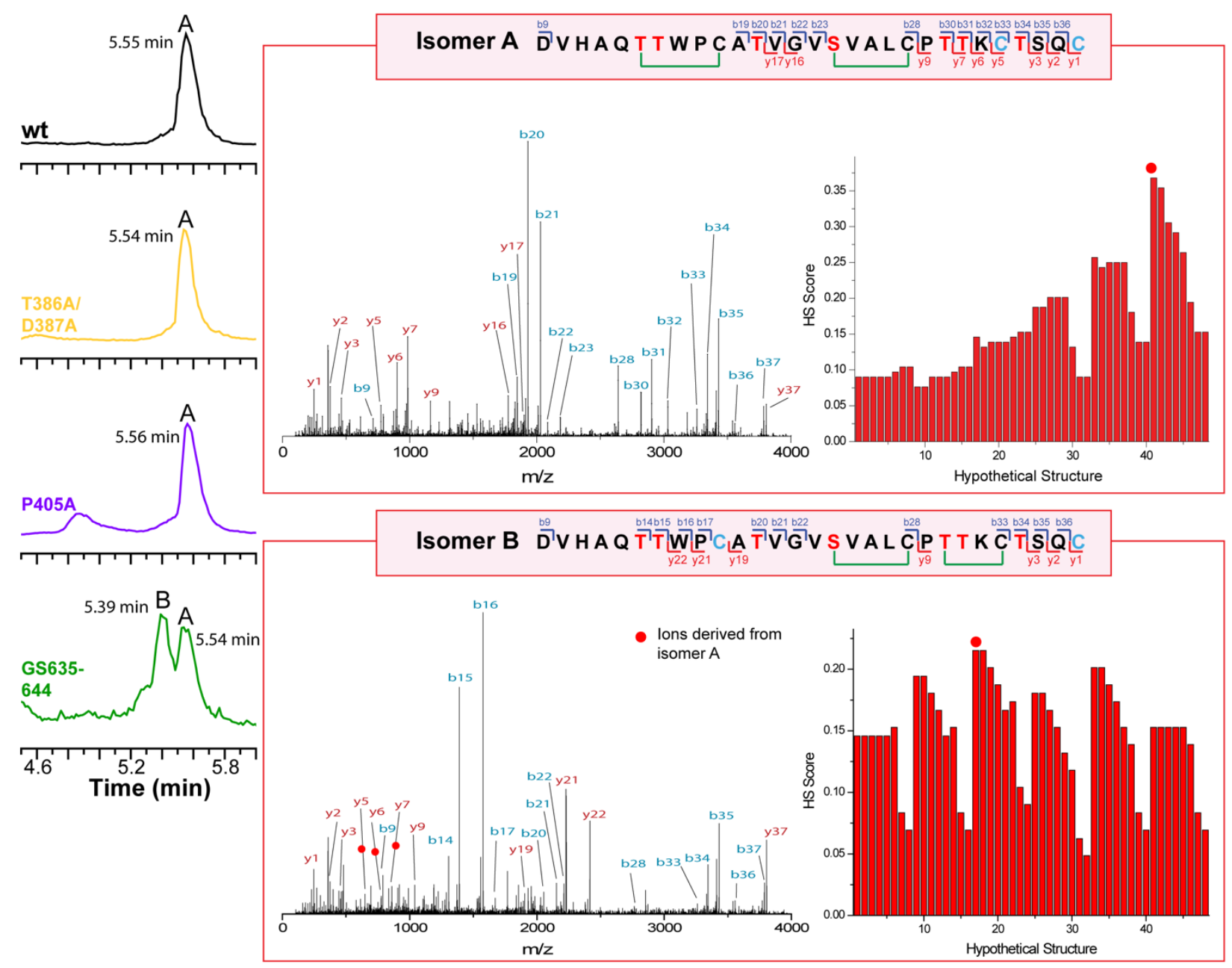

Figure S19. Retention time analysis and tandem mass spectrometry for GluC-digested HalA2 species containing two thioether rings and five additional dehydrations, [2L5D]. The $4^{+}$ion of [2L5D] was chosen for fragmentation $(\mathrm{m} / \mathrm{z}=951.935 \mathrm{Da})$. Extracted ion chromatograms (EICs) for [2L5D] produced in the reactions of each HalM2 enzyme are shown on the left, with the various cyclization isomers labeled A-B. On the right are shown annotated tandem mass spectra for each cyclization isomer along with HSEE predictions. The highest scoring structure in the HSEE analysis is indicated with a red dot, and a model of this structure is shown above the data for each isomer. In the model, the dehydrated Ser/Thr resides are colored red and NEM-alkylated Cys residues are colored blue. The thioether ring topology and $b$ and $y$ fragment ions observed in the tandem mass spectrum are indicated. Each isomer appears to be fully dehydrated at the native positions. The spectrum for isomer $A$ is taken from the HalM2 $2_{w t}$ reaction. The spectrum 
for isomer B (taken from the HalM2 ${ }_{G S 635-644}$ reaction) appears to have contaminating fragment ions derived from isomer $\mathrm{A}$ (red dots). 

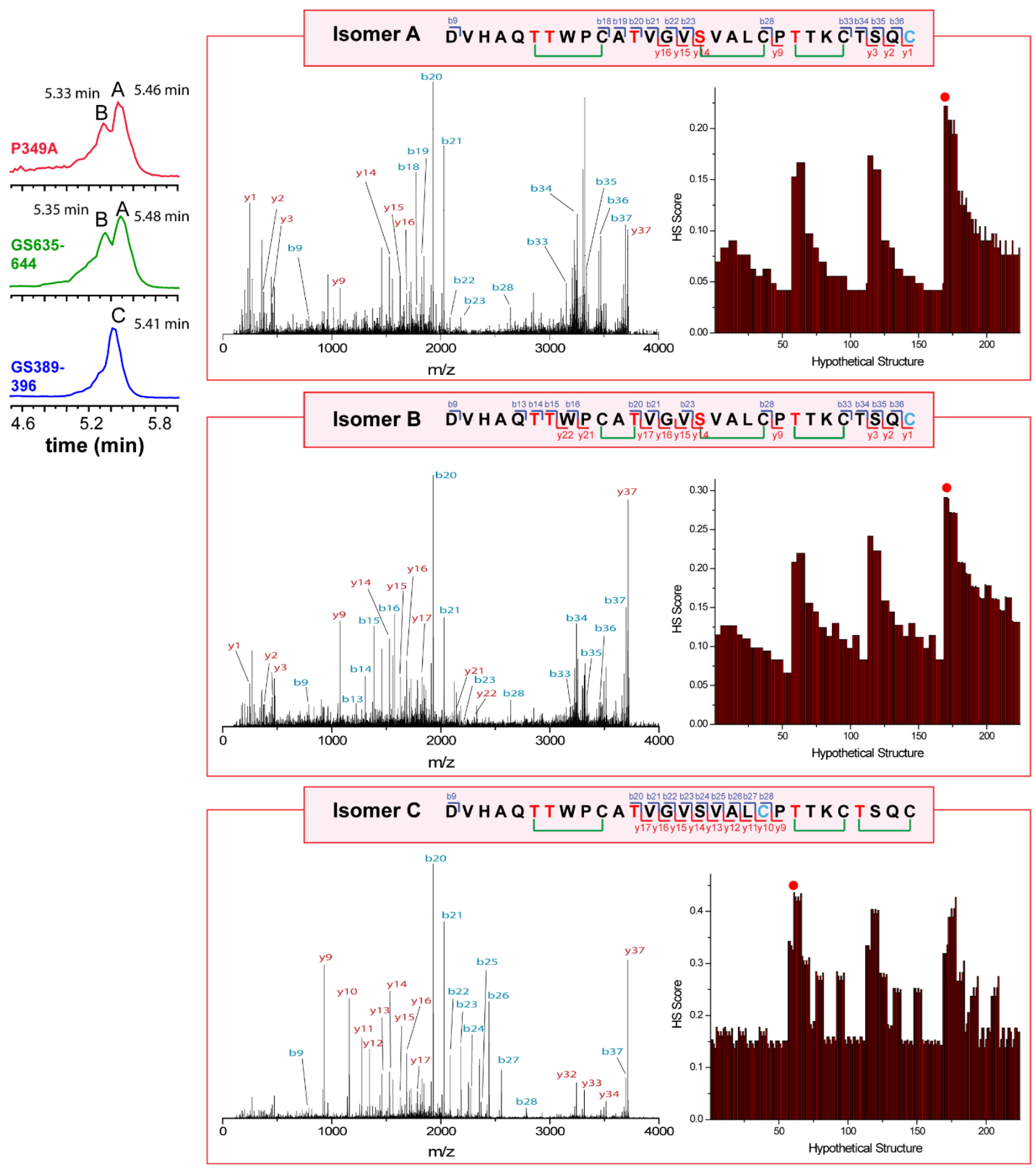

Figure S20. Retention time analysis and tandem mass spectrometry for GluC-digested HalA2 species containing three thioether rings and two additional dehydrations, [3L2D]. The $4^{+}$ion of [3L2D] was chosen for fragmentation $(\mathrm{m} / \mathrm{z}=929.679 \mathrm{Da})$. Extracted ion chromatograms (EICs) for [3L2D] produced in the reactions of each HalM2 enzyme are shown on the left, with the various cyclization isomers labeled A-C. On the right are shown 
annotated tandem mass spectra for each cyclization isomer along with HSEE predictions. The highest scoring structure in the HSEE analysis is indicated with a red dot. In the model, the dehydrated Ser/Thr resides are colored red and NEM-alkylated Cys residues are colored blue. The thioether ring topology and $b$ and $y$ fragment ions observed in the tandem mass spectrum are indicated. The spectrum of isomer $A$ (shown for the HalM2Gs635-644 reaction) suggests the presence of rings A-B-C. The spectrum of isomer $B$ (taken from the HalM2GS635-644 reaction) indicates the presence of rings B-C and a nonnatural ring formed between Cys5-Thr7. This assignment is supported by the presence of $b_{14-16}$ and $y_{21-22}$ ions (indicating lack of native ring A), and by the NEM-alkylated $y_{1-3}$ ions (indicating that the final Cys is not engaged in a thioether linkage). The spectrum of isomer $\mathrm{C}$ (taken from the HalM2GS389-396 reaction) indicates a unique A-C-D topology not observed in any of the other reactions. 

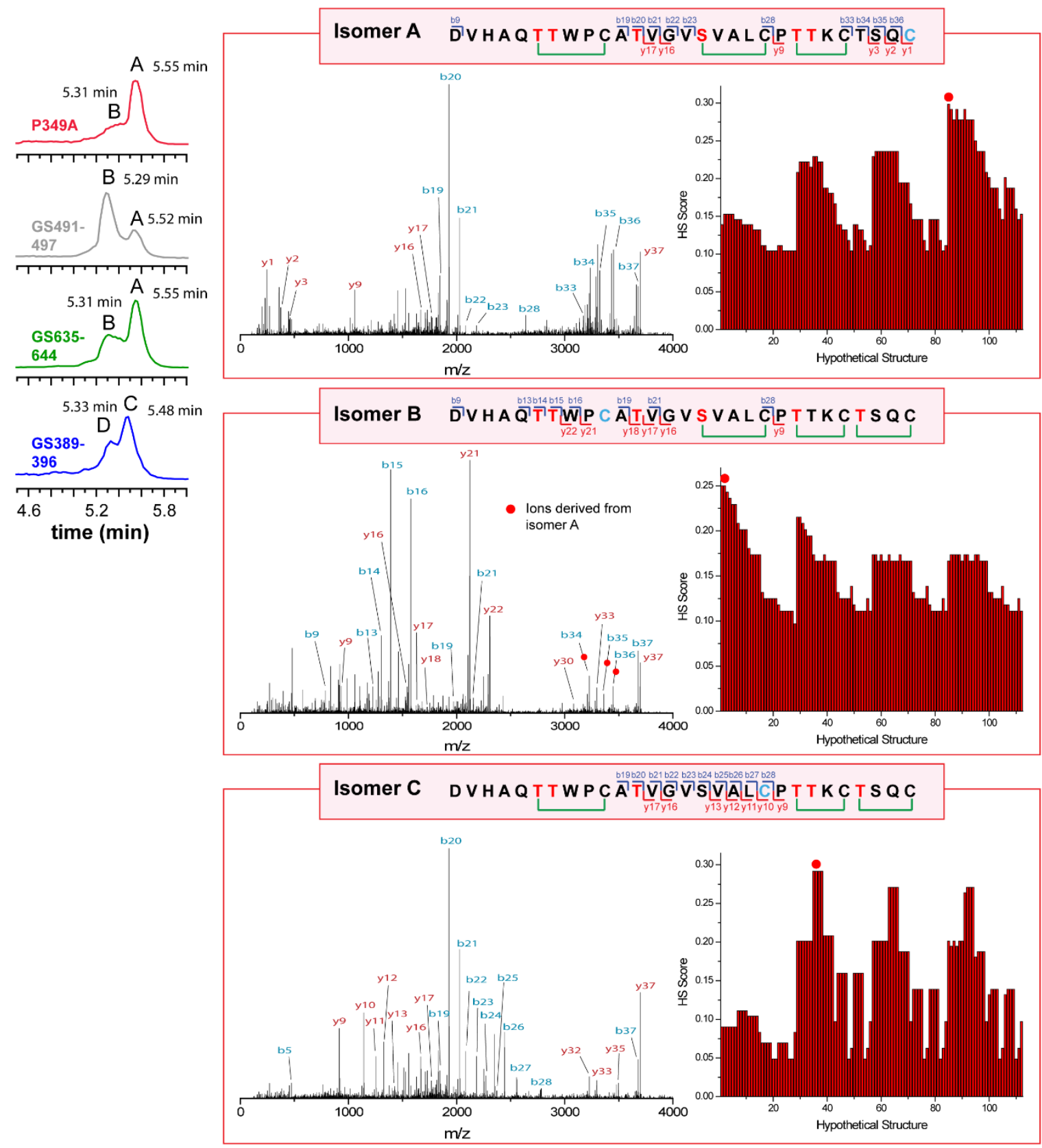

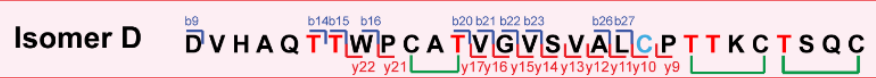

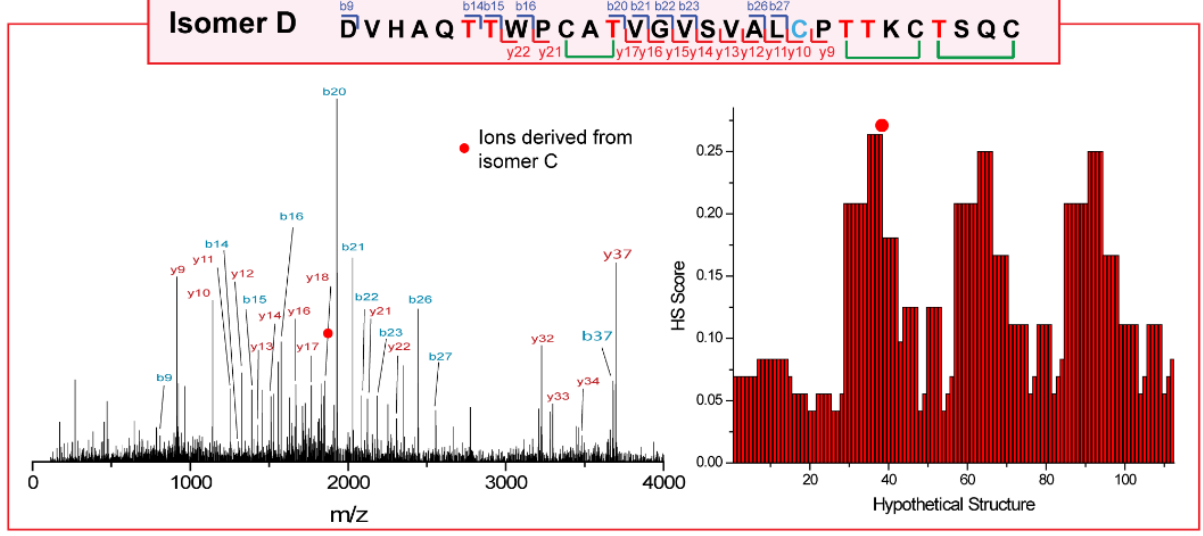


Figure S21. Retention time analysis and tandem mass spectrometry for GluC-digested HalA2 species containing three thioether rings and three additional dehydrations, [3L3D]. The $4^{+}$ion of [3L3D] was chosen for fragmentation $(\mathrm{m} / \mathrm{z}=925.176 \mathrm{Da})$. Extracted ion chromatograms (EICs) for [3L3D] produced in the reactions of each HalM2 enzyme are shown on the left, with the various cyclization isomers labeled A-D. On the right are shown annotated tandem mass spectra for each cyclization isomer along with HSEE predictions. The highest scoring structure in the HSEE analysis is indicated with a red dot. In the model, the dehydrated Ser/Thr resides are colored red and NEM-alkylated Cys residues are colored blue. The thioether ring topology and $b$ and $y$ fragment ions observed in the tandem mass spectrum are indicated. The spectrum of Isomer $A$ is taken from the HalM2 ${ }_{P 349 A}$ reaction. The spectrum of isomer B is taken from the HalM2 ${ }_{G S 491-497}$ reaction. The spectra for isomers $C$ and $D$ are both derived from the HalM2GS389-396 reaction. Isomer $\mathrm{D}$ appears to contain a non-native thioether ring formed between Cys5-Thr7. Evidence for this assignment is provided by the strong signals for the NEM-alkylated $y_{10-17}$ ions (indicating that the free Cys thiol is C-terminal to Cys5), along with the $b_{14-16}$ and $y_{21-22}$ ions (that indicate lack of the native ring $\mathrm{A}$ ). 

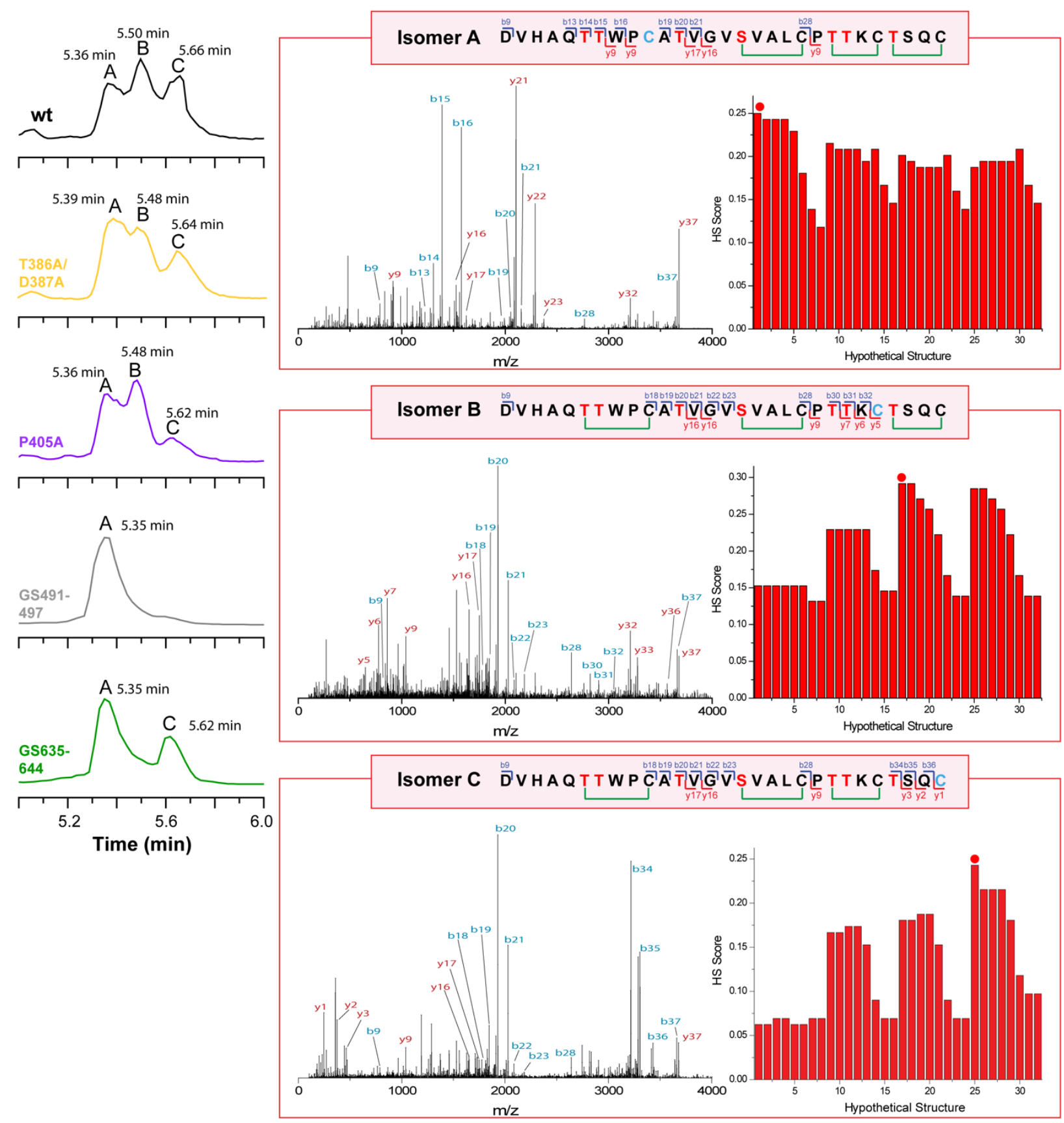

Figure S22. Retention time analysis and tandem mass spectrometry for GluC-digested HalA2 species containing three thioether rings and four additional dehydrations, [3L4D]. The $4^{+}$ion of [3L4D] was chosen for fragmentation $(\mathrm{m} / \mathrm{z}=920.430 \mathrm{Da})$. Extracted ion chromatograms (EICs) for [3L4D] produced in the reactions of each HalM2 enzyme are shown on the left, with the various cyclization isomers labeled A-C. On the right are shown annotated tandem mass spectra for each cyclization isomer along with HSEE predictions. 
The highest scoring structure in the HSEE analysis is indicated with a red dot. In the model, the dehydrated Ser/Thr resides are colored red and NEM-alkylated Cys residues are colored blue. The thioether ring topology and $b$ and $y$ fragment ions observed in the tandem mass spectrum are indicated. The spectrum of Isomer A is taken from the HalM2P349A reaction. The spectrum of isomer B is taken from the HalM2 ${ }_{G S 491-497}$ reaction. The spectrum for isomer $\mathrm{C}$ is derived from the HalM2GS389-396 reaction. 


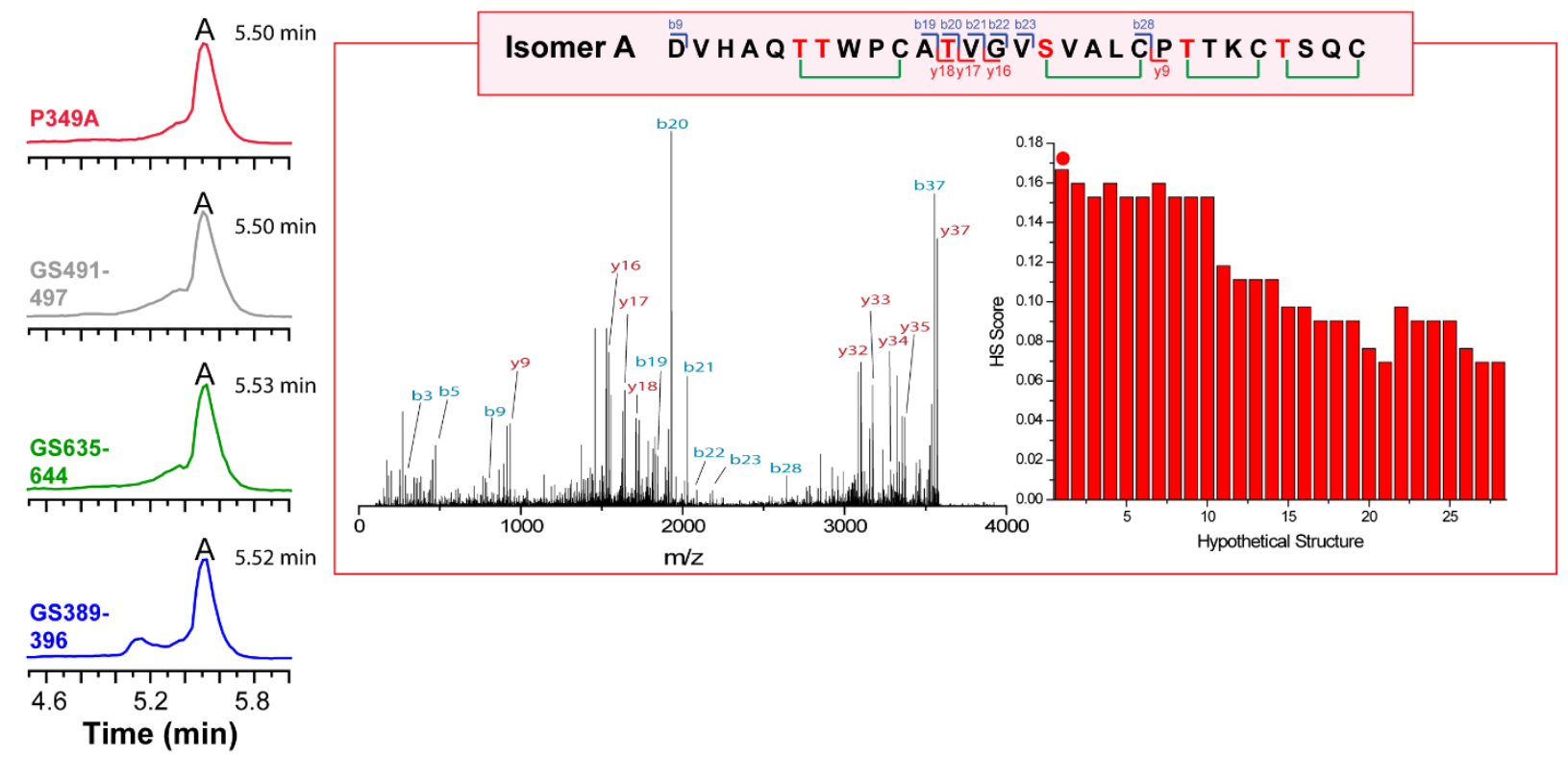

Figure S23. Retention time analysis and tandem mass spectrometry for GluC-digested HalA2 species containing four thioether rings and two additional dehydrations, [4L2D]. The $4^{+}$ion of [4L2D] was chosen for fragmentation $(\mathrm{m} / \mathrm{z}=893.914 \mathrm{Da})$. Extracted ion chromatograms (EICs) for [4L2D] produced in the reactions of each HalM2 enzyme are shown on the left, with the single cyclization isomer labeled $A$. On the right are shown annotated tandem mass spectra for each cyclization isomer along with HSEE predictions. The highest scoring structure in the HSEE analysis is indicated with a red dot, and a model of this structure is shown above the data for each isomer. In the model, the dehydrated Ser/Thr resides are colored red and NEM-alkylated Cys residues are colored blue. The thioether ring topology and $b$ and $y$ fragment ions observed in the tandem mass spectrum are indicated. The spectrum from the HalM2P349A variant is shown. In each case, the Thr18 residue of the core peptide eludes dehydration. 


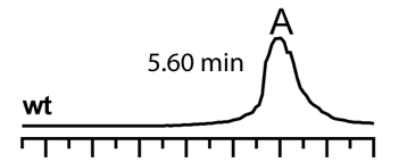

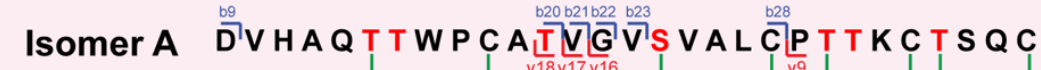
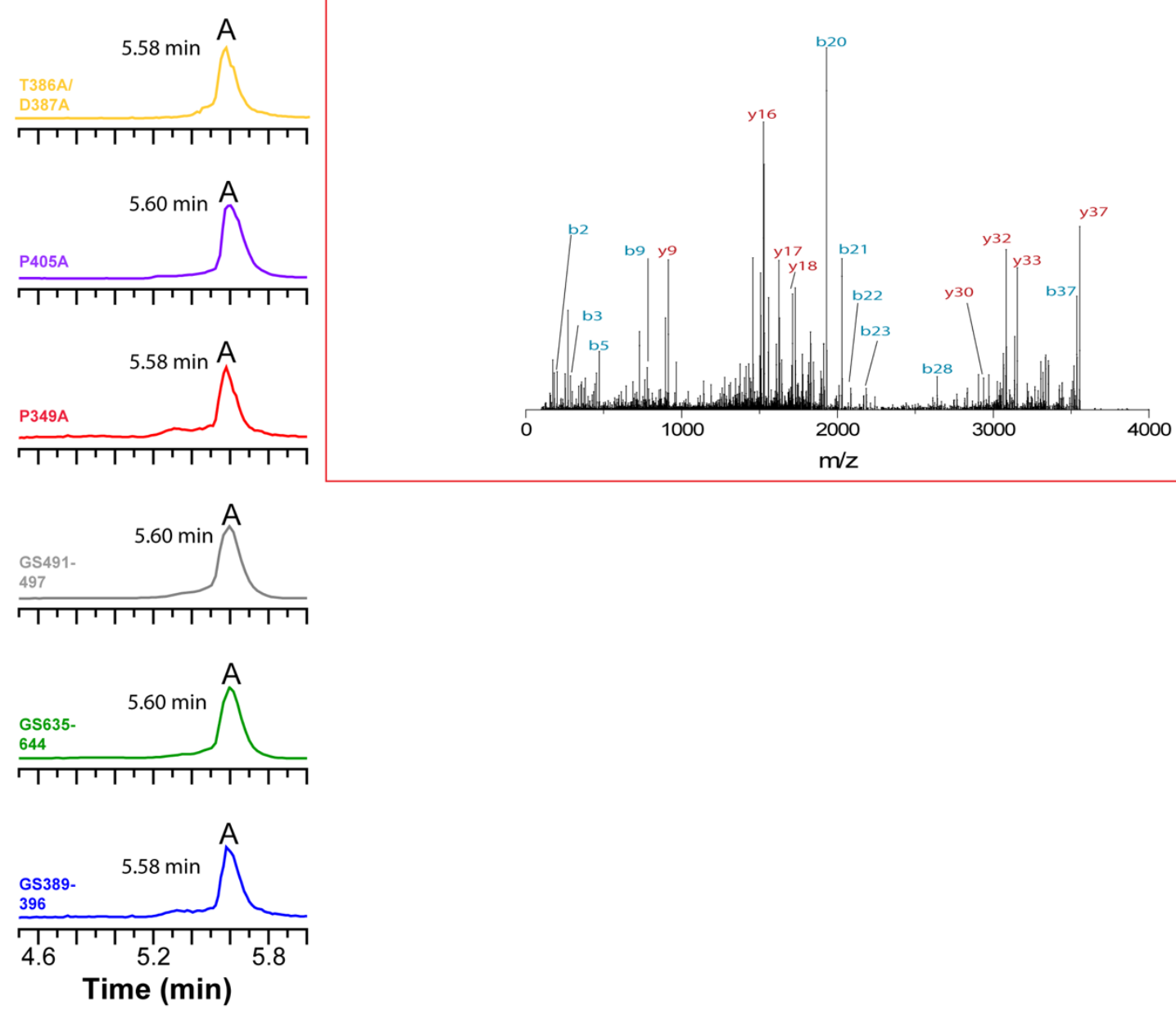

Figure S24. Retention time analysis and tandem mass spectrometry for GluC-digested HalA2 species containing four thioether rings and three additional dehydrations, [4L3D]. The $4^{+}$ion of [4L3D] was chosen for fragmentation $(\mathrm{m} / \mathrm{z}=889.412 \mathrm{Da})$. Extracted ion chromatograms (EICs) for [4L3D] produced in the reactions of each HalM2 enzyme are shown on the left, with the single cyclization isomer labeled $A$. On the right are shown annotated tandem mass spectra for each cyclization isomer along with HSEE predictions. The highest scoring structure in the HSEE analysis is indicated with a red dot, and a model of this structure is shown above the data for each isomer. In the model, the dehydrated Ser/Thr resides are colored red and NEM-alkylated Cys residues are colored blue. The thioether ring topology and $b$ and $y$ fragment ions observed in the tandem mass 
spectrum are indicated. Each HalM2 variant appeared to form the same product with the native ring topology. 

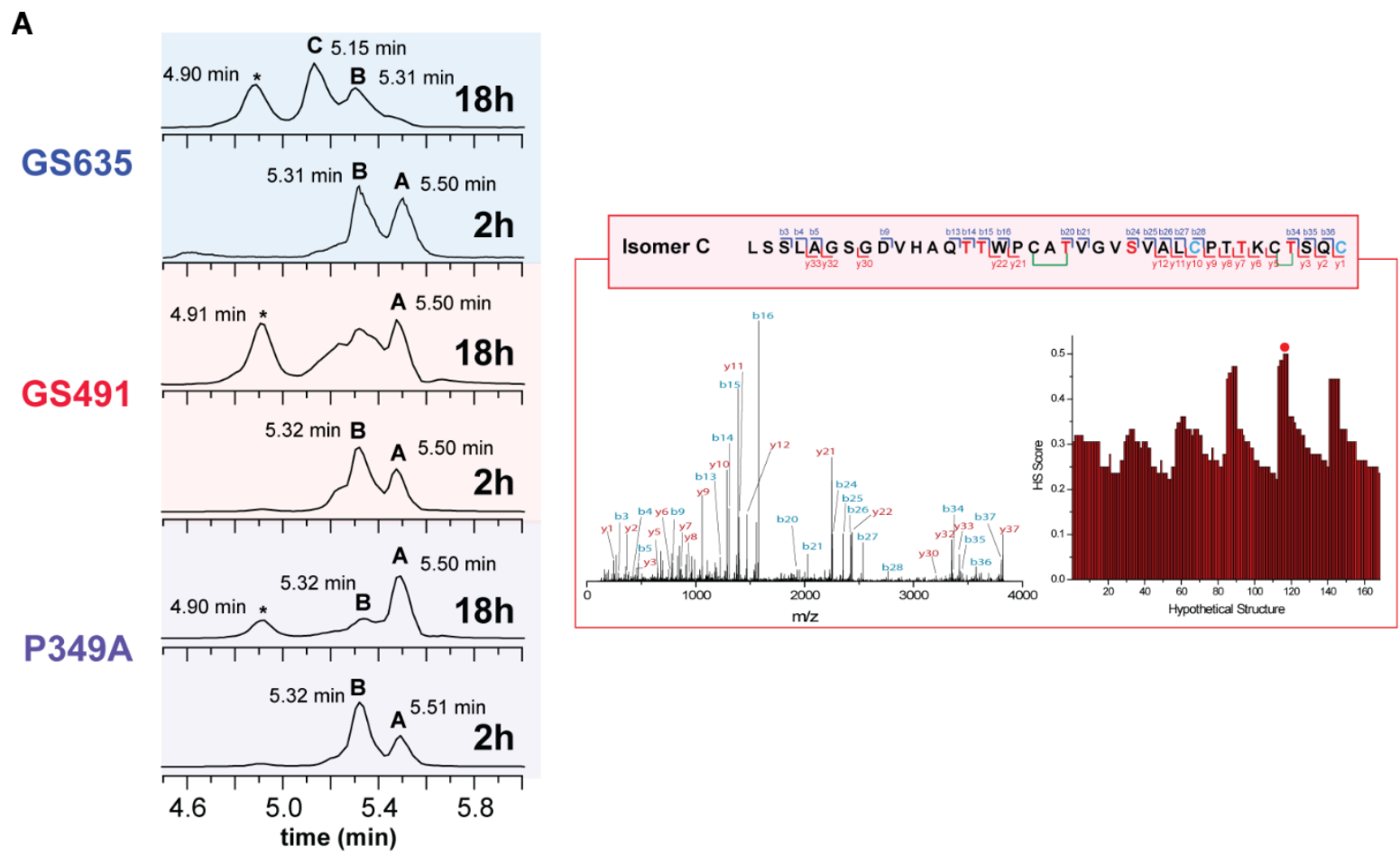

B
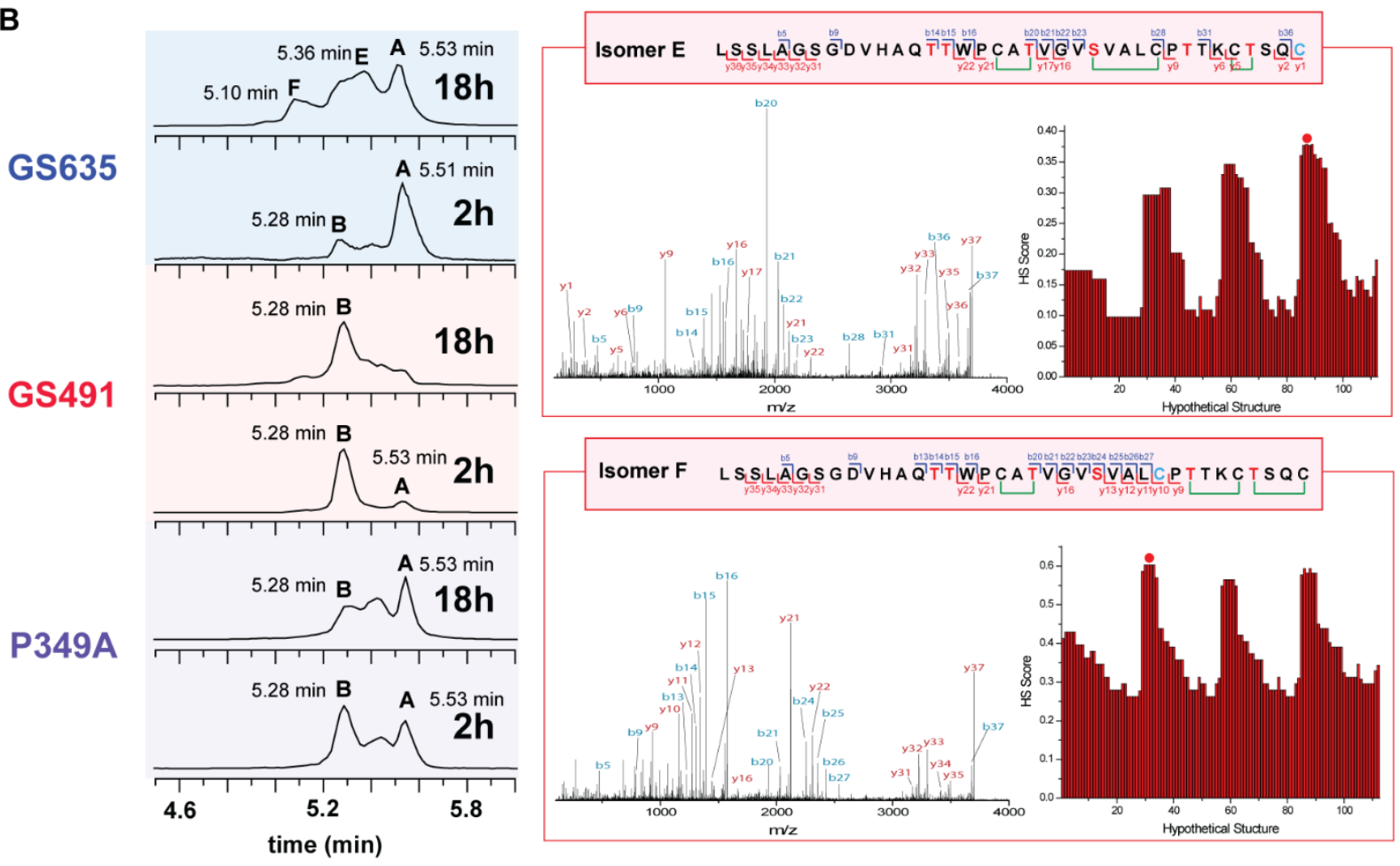

Figure S25. Unusual HalA2 cyclization topologies produced by the HalM2Gs635-644, HalM2Gs491-497, HalM2P349A enzymes. A) The EICs for the $4^{+}$ions of the 2L4D species $(\mathrm{m} / z=956.438 \mathrm{Da})$ show the formation of additional isomers upon extended $(18 \mathrm{~h})$ incubation with each enzyme. The thioether topology of the unusual isomer $\mathrm{C}$ produced 
by HalM2 ${ }_{G S 635-644}$ could be assigned by tandem MS and HSEE analysis. The nearly complete $y 1-y 12$ ion series suggests a lack of native rings $\mathrm{B}, \mathrm{C}$, and $\mathrm{D}$, and that $\mathrm{Cys} 20$ is engaged in a thioether linkage. The $y 4$ ion was not detectable, suggesting a nonnatural thioether ring between Cys20 and the adjacent Thr21 residue. The strong b14$b 16$ ions suggest that ring $A$ is also unnatural and likely forms between Cys 5 and Thr 7 . The thioether topologies of isomers $A$ and $B$ are assigned in Figure S18. The species marked with an asterisk is a TCEP adduct of the fully cyclized product. B) EICs and fragmentation data for the $4^{+}$ions of the 3 L3D species $(\mathrm{m} / z=925.176 \mathrm{Da})$, showing accumulation of additional isomers $(E$ and $F)$ upon prolonged incubation (18 h). For isomer E, HSEE predicted a structure with the same two unnatural rings observed in 2L4D isomer $\mathrm{C}$ (see panel $\mathrm{A}$ ) along with the native ring $\mathrm{B}$. HSEE analysis suggested that isomer $F$ contains native rings $C$ and $D$, along with an unnatural ring $A$ formed by cyclization of Cys 5 onto Thr7. This prediction is supported by the extensive $b 13-b 27$ ion series lacking only the $b 18-b 19$ ions within the putative ring, and the clear alkylation of Cys 15. 


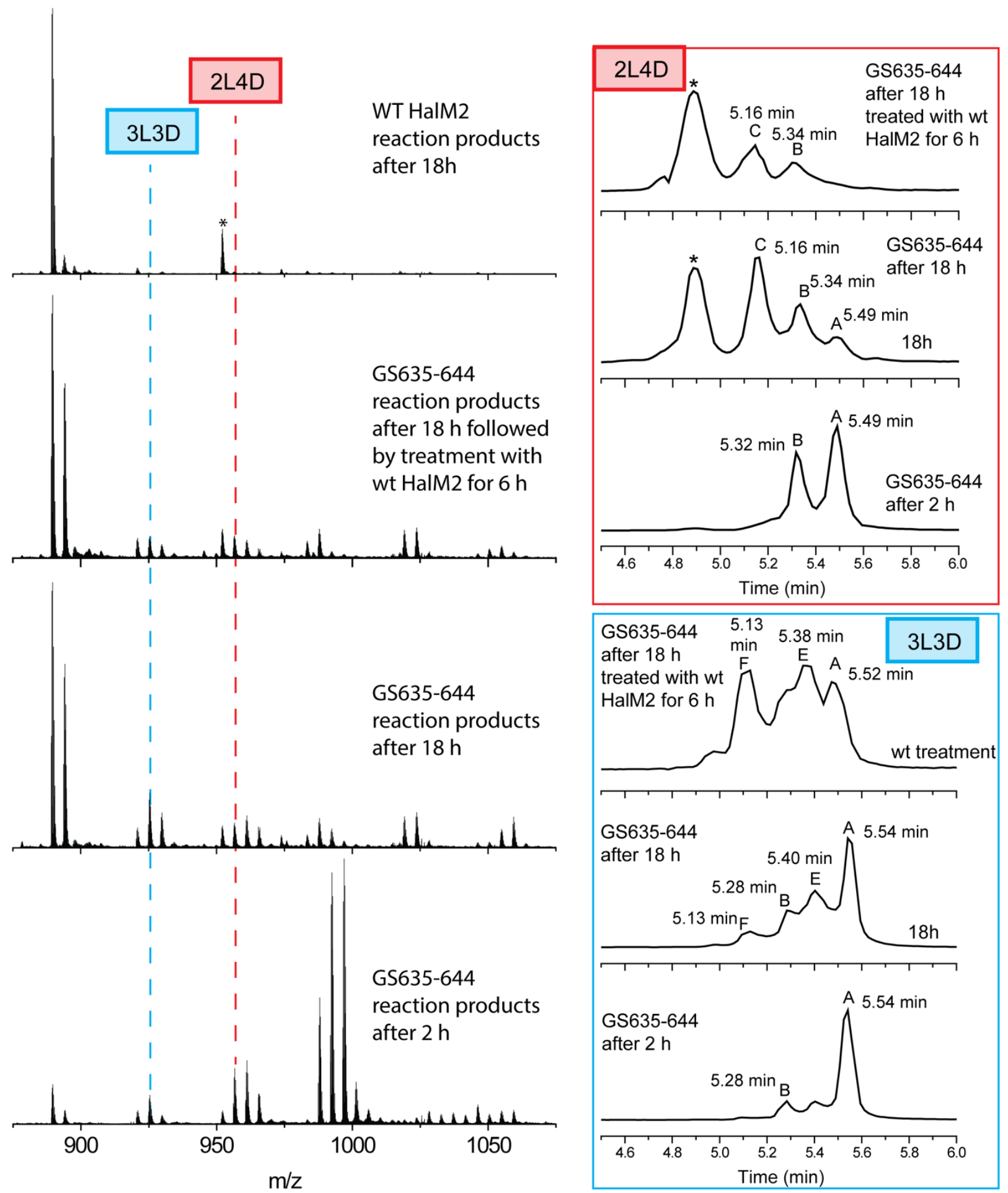

Figure S26. Wt HalM2 cannot correct unnatural thioether topologies. To the left are shown ESI-MS spectra for the $4^{+}$charge state of GluC-digested HalM2Gs635-644 reaction products after $2 \mathrm{~h}, 18 \mathrm{~h}$, and $18 \mathrm{~h}$ followed by treatment with wt HalM2. The reaction products generated by wt HalM2 after $18 \mathrm{~h}$ are shown for comparison in the top spectrum. 
Treatment of the HalM2GS635-644 $18 \mathrm{~h}$ reaction products with wt HalM2 for $6 \mathrm{~h}$ resulted in minimal progression of the reaction, suggesting the presence of dead-end species that are not competent substrates for HalM2. EICs for the 2L4D and 3L3D species produced in these reactions are shown to the right. Over time, improperly cyclized products (isomer $C$ for the 2L4D intermediate and isomers $E$ and $F$ for the 3L3D intermediate - see Figure S25) become more apparent in the HalM2GS635-644 reactions as the natively cyclized isomers are consumed. Treatment of these samples with wt HalM2 results in the further consumption of the natively cyclized isomers and the persistence of the improperly cyclized isomers. These data show that certain incorrect thioether topologies cannot be corrected by the wt HalM2 and suggest that the cyclization reactions may be under kinetic control. 

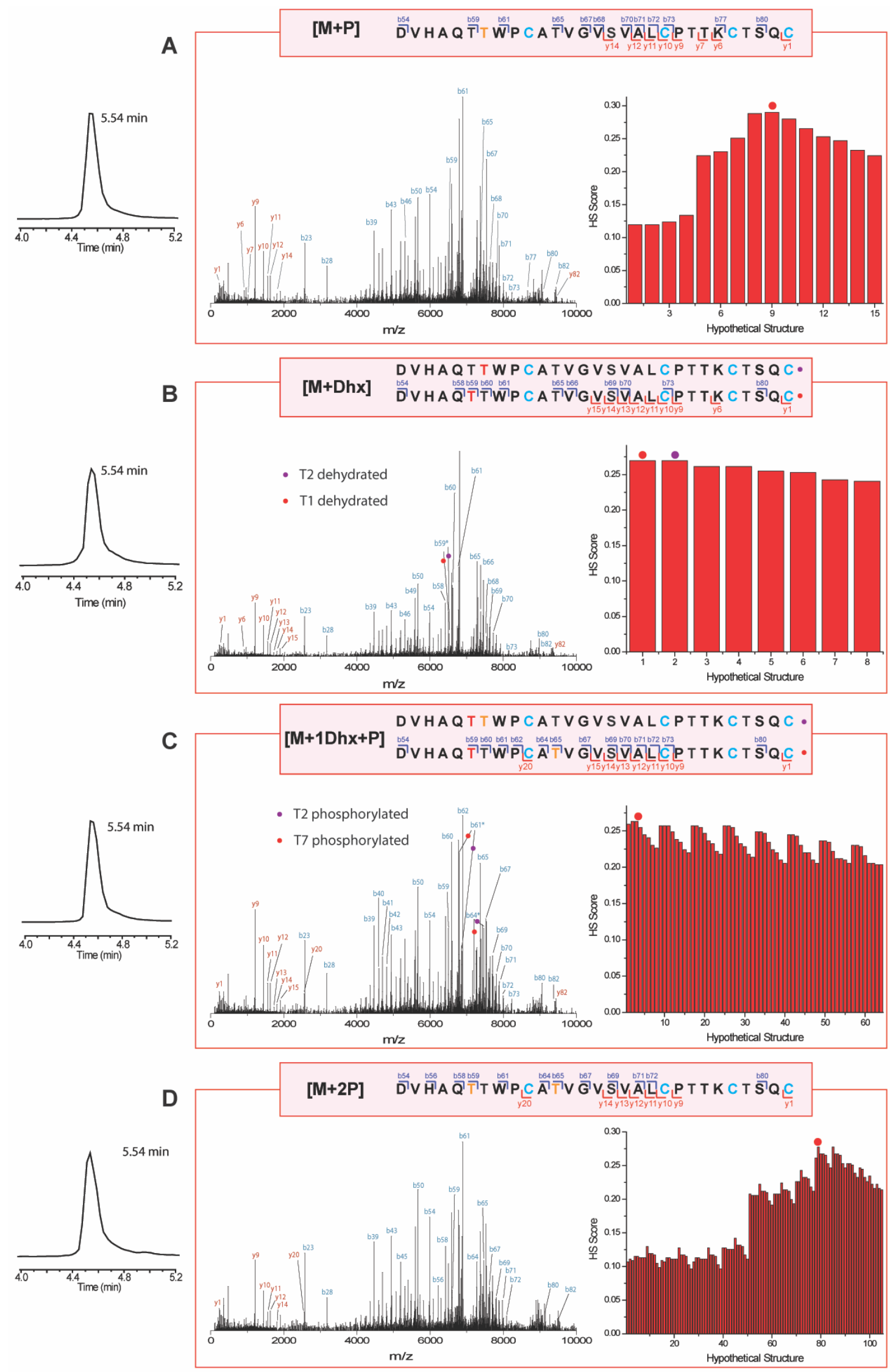
Figure S27. Tandem MS and HSEE analysis of selected reaction intermediates produced by the HalM2 ${ }_{\mathrm{GS} 360-365}$ variant. The extracted ion chromatograms and retention times for each intermediate are shown on the left. Annotated tandem mass spectra, generated by fragmentation of the $8^{+}$ions of the undigested peptides are shown on the right, along with HSEE predictions. The highest scoring structure in the HSEE analysis is indicated with a red dot, and a model of this structure is shown above the data for each isomer. In the models, the dehydrated Ser/Thr resides are colored red, phosphorylated Ser/Thr residues are colored orange and NEM-alkylated Cys residues are colored blue. Shown are data for (panel $\mathbf{A})$ the singly phosphorylated species, $[\mathrm{M}+\mathrm{P}](\mathrm{m} / \mathrm{z}=$ $1183.81 \mathrm{Da}$ ), (panel B) the singly dehydrated species, [M+Dhx] $(\mathrm{m} / z=1171.56 \mathrm{Da})$, (panel C) species [M+Dhx+P] $(m / z=1181.56 D a)$, and (panel D) the doubly phosphorylated species, $[\mathrm{M}+2 \mathrm{P}](\mathrm{m} / z=1193.81 \mathrm{Da})$. All of the modifications are clustered in the $\mathrm{N}$-terminal region of the HalA2 core peptide, and no significant cyclization activity was observed with this variant. 


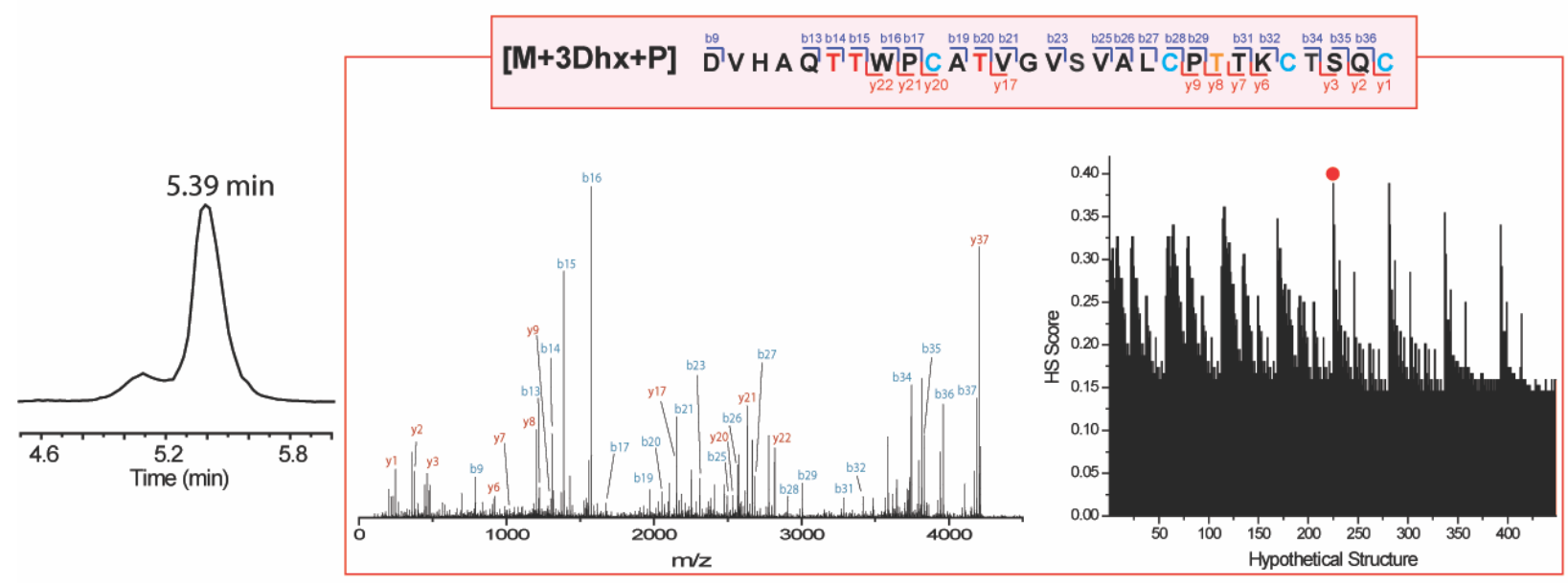

Figure S28. Tandem MS analysis of the [3D1P] phosphorylated intermediate produced by HalM2GS389-396. The sample was GluC-digested and the $4^{+}$ion was chosen for fragmentation $(m / z=1052.46 \mathrm{Da})$. The extracted ion chromatogram is shown on the left. The tandem mass spectrum and HSEE predictions are shown to the right. The highest scoring hypothetical structure is indicated with a red dot, and a model of this structure is shown. In the model, the dehydrated Thr resides are colored red, the phosphorylated Thr is colored orange, and the NEM-alkylated Cys residues are colored blue. The data indicate that there is a preference for dehydrating Thr1, Thr2, and Thr7, but that dehydration of Ser11 is skipped. 

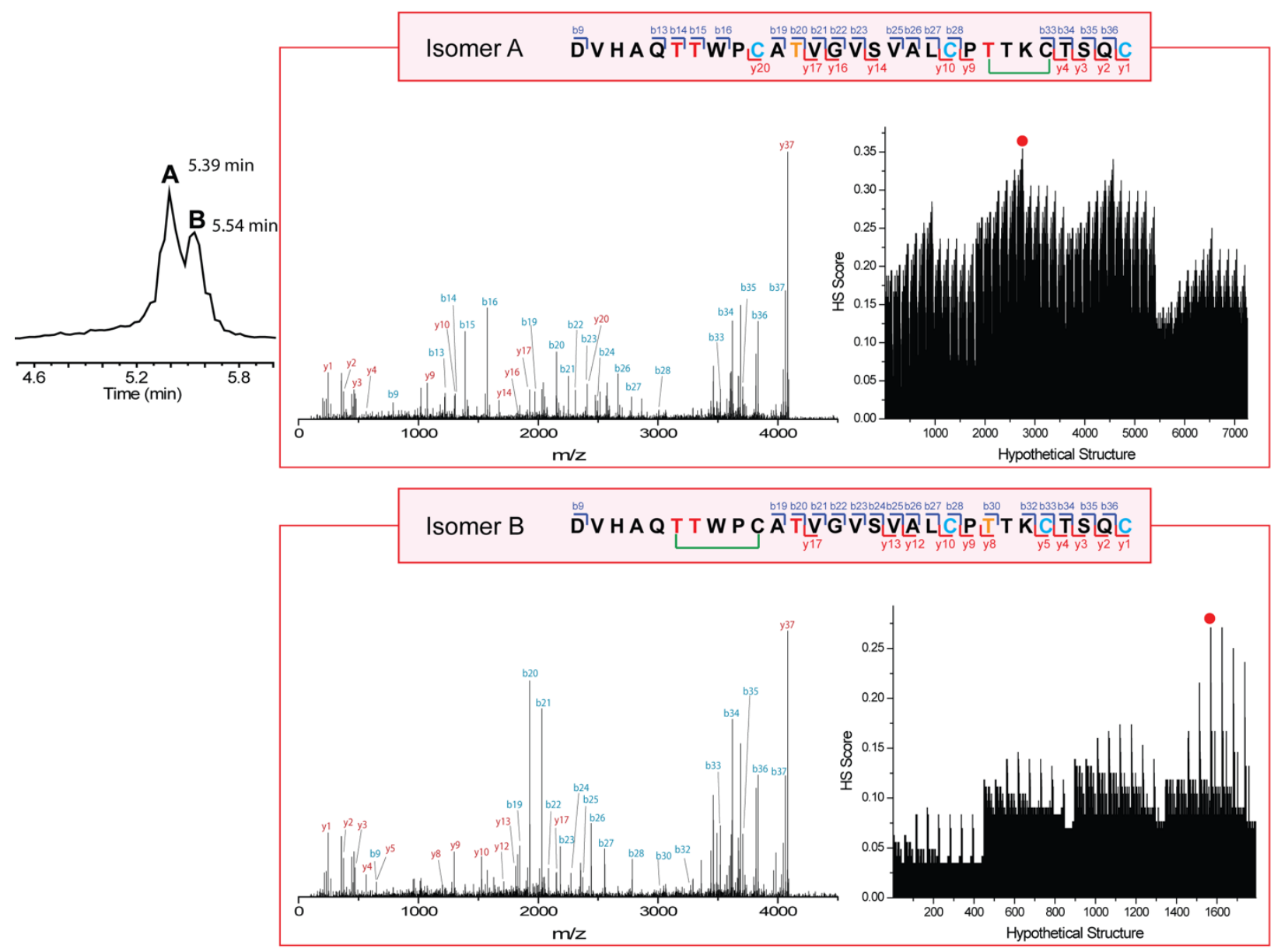

Figure S29. Tandem MS analysis of the [1L2D1P] phosphorylated intermediates produced by HalM2Gs389-396. The sample was GluC-digested and the $4^{+}$ion was chosen for fragmentation $(\mathrm{m} / \mathrm{z}=1021.12 \mathrm{Da})$. The extracted ion chromatogram is shown on the left. The tandem mass spectrum and HSEE predictions are shown to the right. The highest scoring hypothetical structure is indicated with a red dot, and a model of this structure is shown. In the model, the dehydrated Thr resides are colored red, the phosphorylated Thr is colored orange, and the NEM-alkylated Cys residues are colored blue. In isomer A, Thr7 is phosphorylated and ring $\mathrm{C}$ is formed. In isomer B, either Thr17 or Thr18 carries the phosphate group, while ring $A$ has formed. These thioether topologies are consistent with those observed in Figures S12-S14 for the singly-cyclized species produced by the HalM2GS389-396 enzyme. These data show that Ser11 eludes dehydration, precluding the installation of thioether ring $B$ that is facile in the reactions catalyzed by the other HalM2 enzymes. 


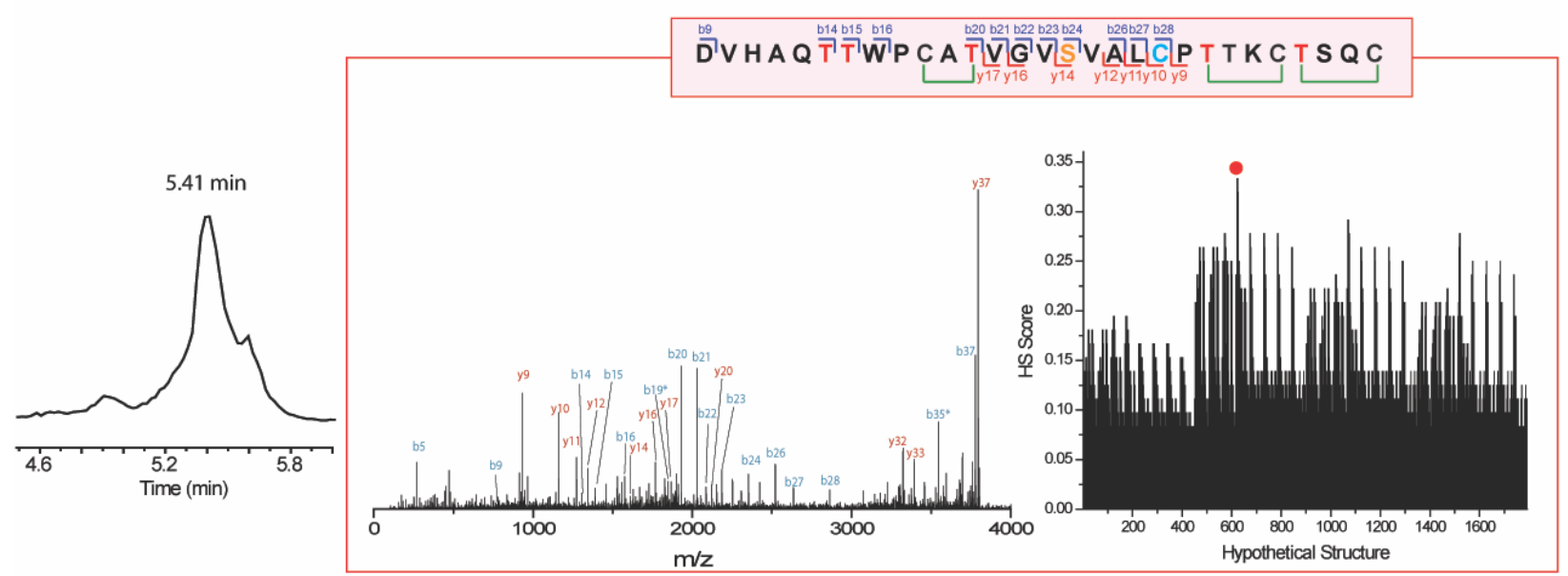

Figure S30. Tandem MS analysis of the [3L2D1P] phosphorylated intermediate produced by HalM2Gs389-396. The sample was GluC-digested and the $4^{+}$ion was chosen for fragmentation $(m / z=949.67 \mathrm{Da})$. The extracted ion chromatogram is shown on the left. The tandem mass spectrum and HSEE predictions are shown to the right. The highest scoring hypothetical structure is indicated with a red dot, and a model of this structure is shown. In the model, the dehydrated Thr resides are colored red, the phosphorylated Thr is colored orange, and the NEM-alkylated Cys residues are colored blue. Isomer $A$ is phosphorylated on Ser11 and contains native rings $C$ and $D$. Though not intense, the presence of the $b_{14-16}$ and $y_{20}$ ions suggest that this species may contain an unnatural thioether linkage between Cys5 and Thr7. This same unnatural topology was observed in the [3L3D] species produced by HalM2GS389-396 (see isomer D, Figure S21). 
A

Deuterium Uptake Difference: [wt HaIM2] - [HalM2 variant]

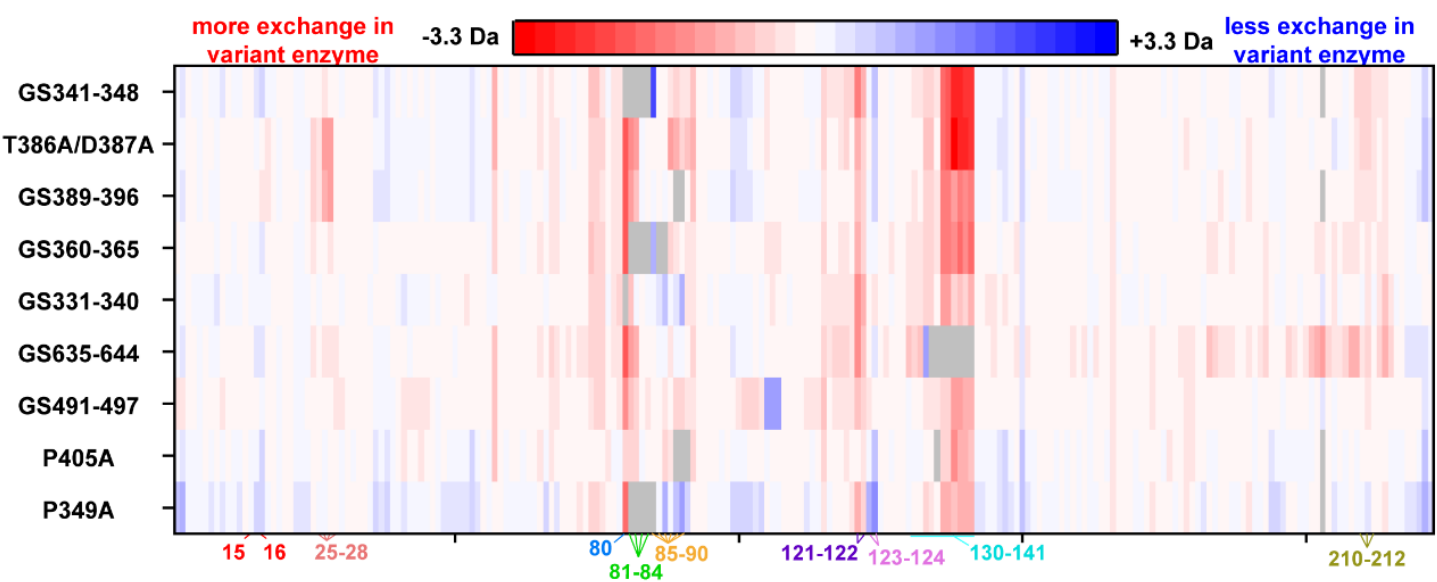

B

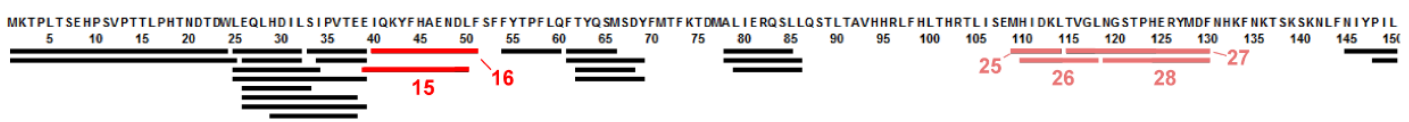

GKLVVNETLRT I MFVKKI IQHYMKDYLLL SDF F KE KDLRLT NLQLGVGDTHVNGQCVT ILTFA SGQKVVYKPRSL SI DKQFGEF IEWWNSKGFQP SLRI PIA I DRQTYGWYEF I PHQEAT SEDE IERYYSR IGGYLA IAYLFGATDLHLD

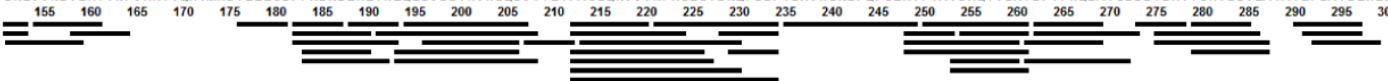

NLIACGE HPML I DLETLFTMDLDCYD SAF PF PALARELTQ SVFGTLMLPIT IA SGKLLDIDL SAVGGGKGVQSEKI KTWW IVNQKTDEMKLVEQPYVTE SSQNKPTVNGKEANIGHYI PHVTDGF RKMYRLF LNE IDELMDHNGPIFAFE

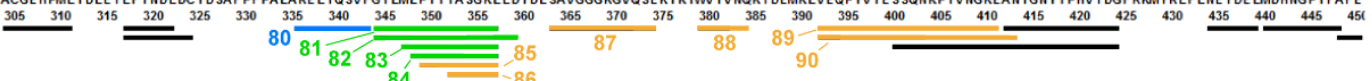

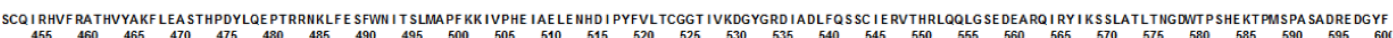

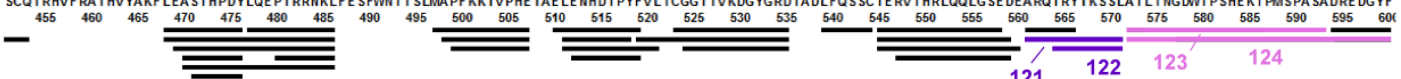

LREAQA IGDD ILAQL IWE DDRHAAYL IGV SVGMNEAVTV SPLTPG IYDGTLG IVLFF DQLAQQTGE THYRHAADALLEGMF KQLKPE LMPS SAYFGLG SLF YGLMVLGLQR SDSH I IQRAYYY YLKHLEECVQHEETPDFV SGL SGVL YML

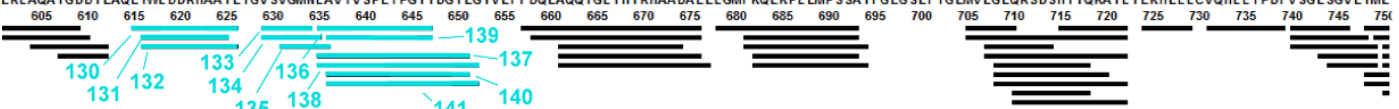
TKI YQLTNE PRVF EVAKTTA SRL SVLLDSKQPDTVLTGL SHGAAGFALALLTYGTAANDEQLLKQGH SYLVYERNRF NKQENMWWDLRKGMAYQTFWCHGAPG IGI SRLLLAQF YDDELLHEELLAALNKTI SDGFGHNH SLCHGDFGNL

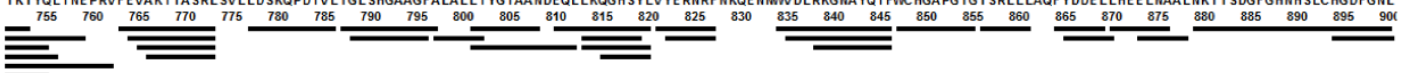
ב=

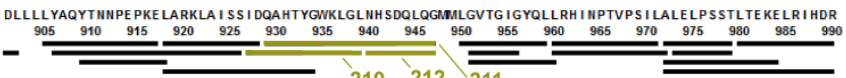
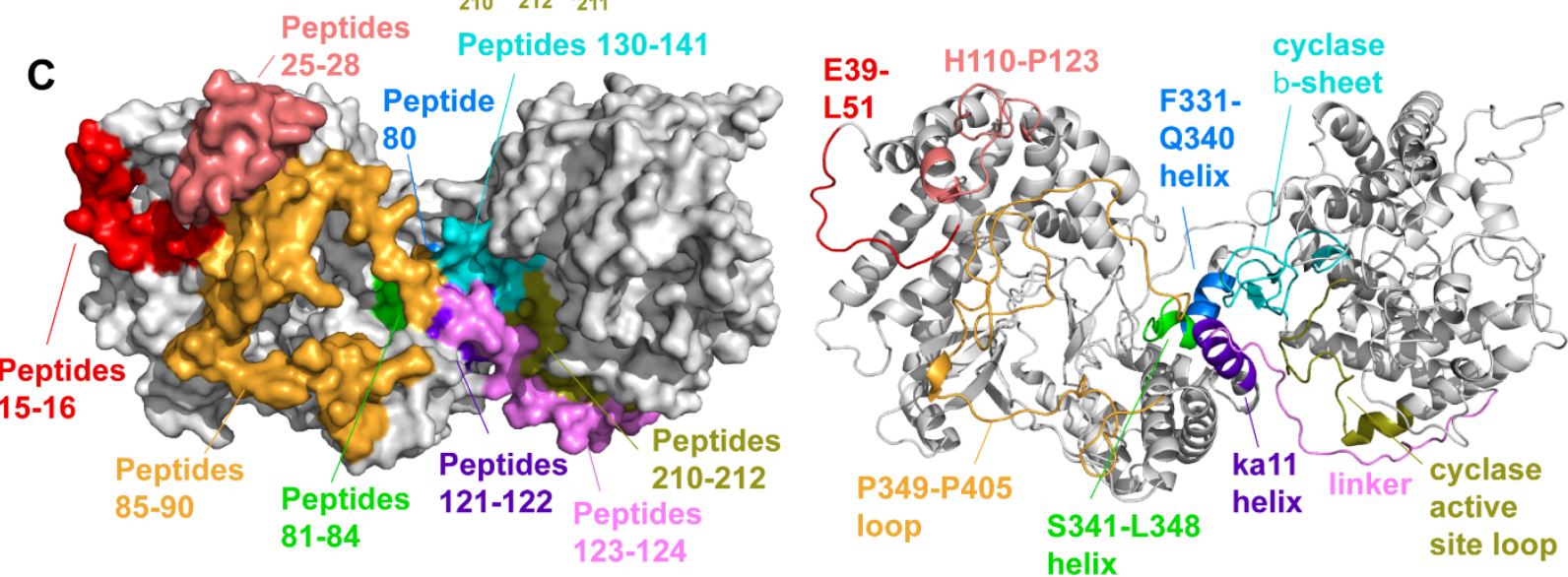
Figure S31. Comparison of deuterium uptake by ligand-free HalM2 variant enzymes relative the ligand-free wt HalM2 enzyme. Deuterium uptake was measured by mass spectrometry for each of 220 peptic peptides spanning $90 \%$ of the HalM2 amino acid sequence. A) The peptide deuterium uptake values for each variant enzyme are subtracted from the uptake values measured for wt HalM2. Overall, the mutations introduce structural flexibility into the variant enzyme, resulting in increased deuterium uptake relative to wt HalM2 (red). B) Coverage map showing the position of each HDX peptide within the primary sequence of HalM2. C) HalM2 homology model showing the location of relevant HDX peptides (left) and the domain nomenclature used in this study (right). All peptides and protein elements in panels $A, B$, and $C$ have been color coded and numbered from 1-220 starting from the $N$-terminus of HalM2. 
MKTPLT SE HP SVPTTLPHTNDT OWLEQLHD ILS I PVTEE IQKYF HAENDLF SFFYTPF LQF TYQ SMSDYFMTF KT DMALIE RQSLLQSTLTAVHHRLFHLTHRTL I SEMH I DKLTVGLNG STPHE RYMDF NHKF NKT SKSKNLLF NIYPIL

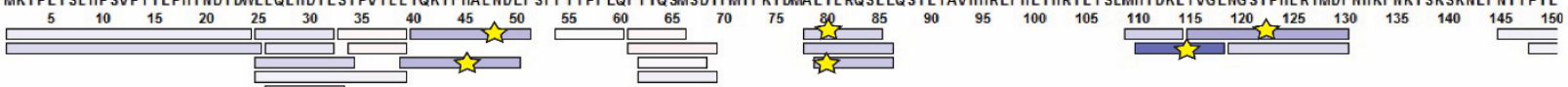

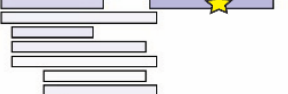

GKLVVNE TLRT INFVKKI IQHYMKDYLLLSDFF KE KDLRLTNLQLGVGDTHVNGQCVTILTTFA SGQKVVYKPR SLSI DKQFGEF IEWWNSKGFQPSLRI PIA I DRQTYGWYEF I PHQEAT SEDE IERYY SRIGGYLA IAYLFGATDLHLD

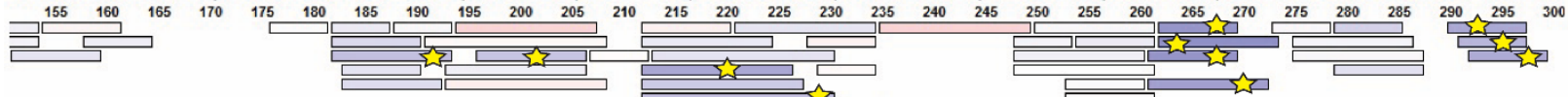
NL IACGE HPML I DLETLFT TNDLDCYD SAF PF PALARE LTQ SVFGTLMLP I I IA SGKLLDIDLSAVGGGKGVQSEK I KTWW IVNQKTDEMKLVEQPYVTE S SQNKPTVNGKEAN IGNY I PHVTDGFF RKMYRLF LNE I DE LMDHNG IFAFE

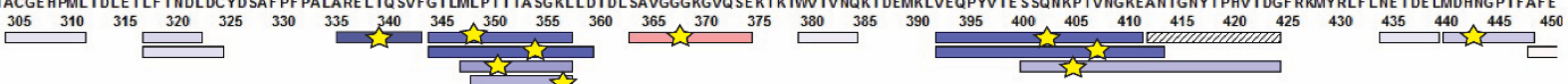
SCQI RHVF RATHUYAKF LEA STHPDYLQE PTRRNKLFE SFWN I T SLMAPF KK IVPHE IAELENHDI PYFVLTTCGGT IVKDGYGRD IADLFQS SC IE RVT HRLQQLG SE DEARQ I RY I KS SLATLT NGDWTP SHE KT PMM SPA SADRE DGYF 455 460 465 LREAQA IGDD I LAQL IWE DDRHAAYL IGV SVGMNEAVTV SPLT PG I YDG TLG IVLFF DQLAQQTGE THYRHAA DALLEGMF KQLKPE LMP S SA YFGLG SLF YGLMVLGLQR SD SH I IQKA YE YLKHLEECVQHEE TPDFV SGL SGVL YML (20)

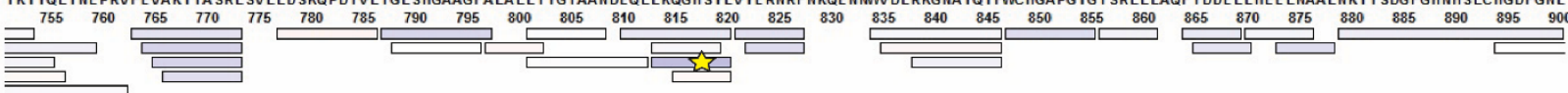

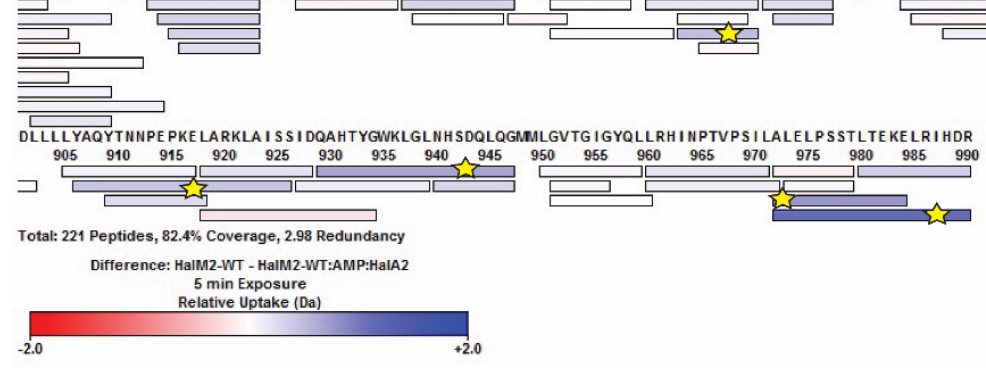

C [HalM2] - [HalM2:HalA2:AMPPNP]

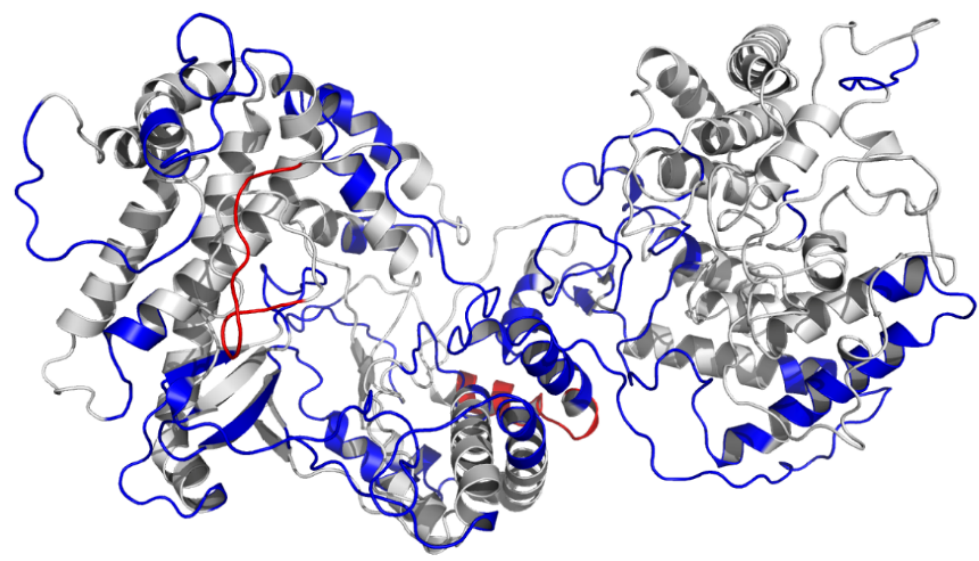

Figure S32. Deuterium uptake difference in peptides derived from wt HalM2 in the presence and absence of AMP-PNP and HalA2 ligands. Each bar represents a different HalM2-derived peptide detected in our HDX-MS workflow. The HalM2 amino acid sequence and residue numbering is indicated in the coverage map. The uptake 
difference was calculated by subtracting the uptake measured in the wt HalM2:AMPPNP:HalA2 Michaelis complex from the uptake measured in the free enzyme. In this representation, the red and blue coloring indicate peptides that uptake more and less deuterium, respectively, upon ligand binding. The peptides marked with a star exhibited a significant uptake difference (|uptake difference $\mid \geq 0.4$ ). C) Deuterium uptake difference in regions of wt HalM2 in the presence and absence of AMP-PNP and HalA2 mapped onto the HalM2 homology model. The uptake difference was calculated by subtracting the uptake measured in the wt HalM2:AMPPNP:HalA2 Michaelis complex from the uptake measured in the free enzyme. A complete list of these peptides and their uptake difference values are provided in Table S6. 


\section{[HalM2 P405A] - [HalM2 P405A:HalA2:AMPPNP]}

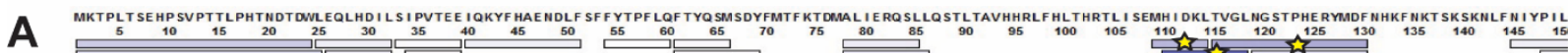

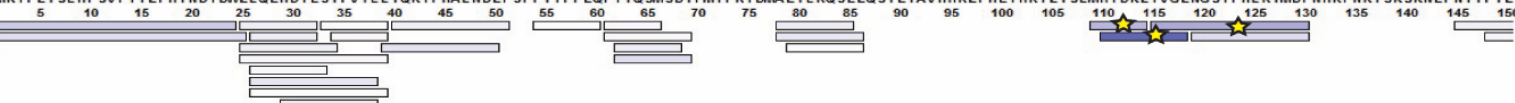
ए人,

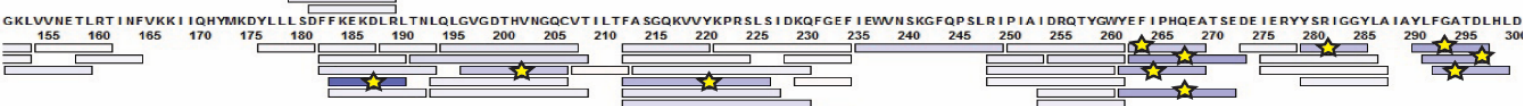

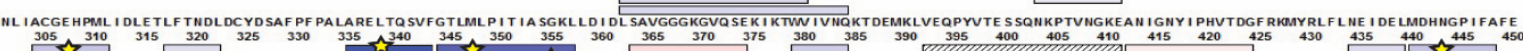
(325

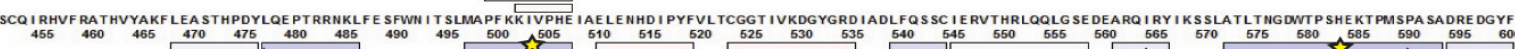
כ 460

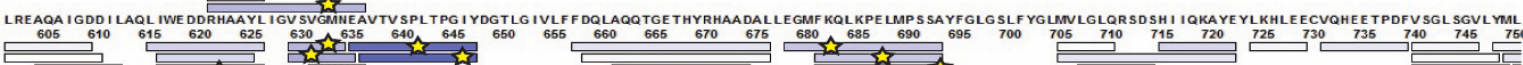
ए स

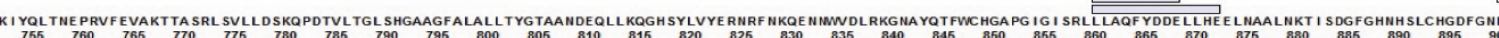
等

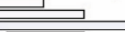

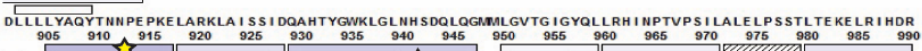
$\sqsupset \stackrel{905}{910}$

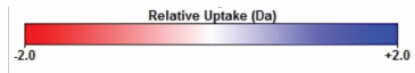

[wt HalM2] - [HalM2 P405A]

B एँ

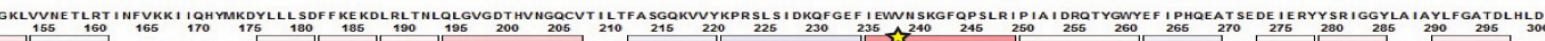

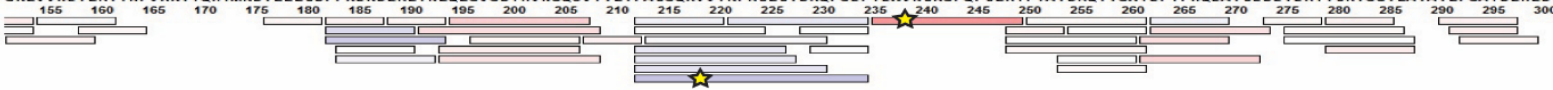

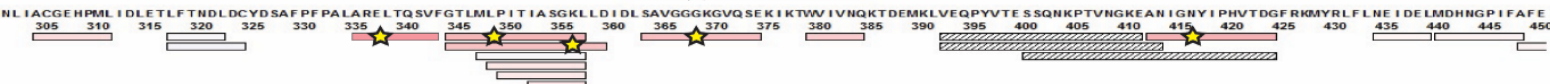

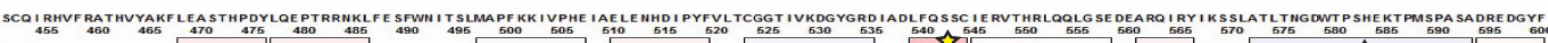

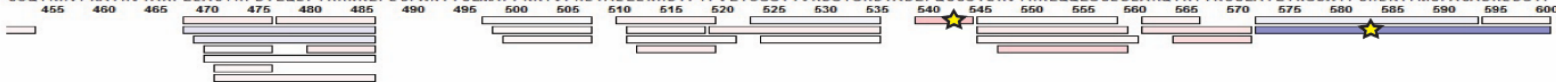

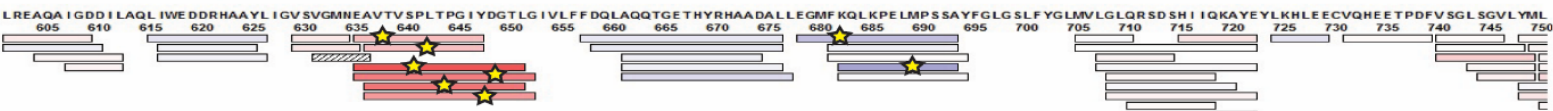

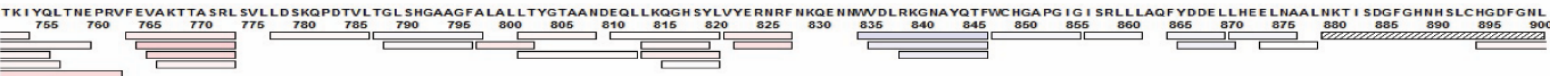
豆

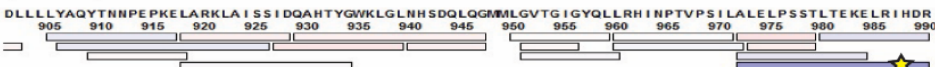

Relative Uptake (Da) 

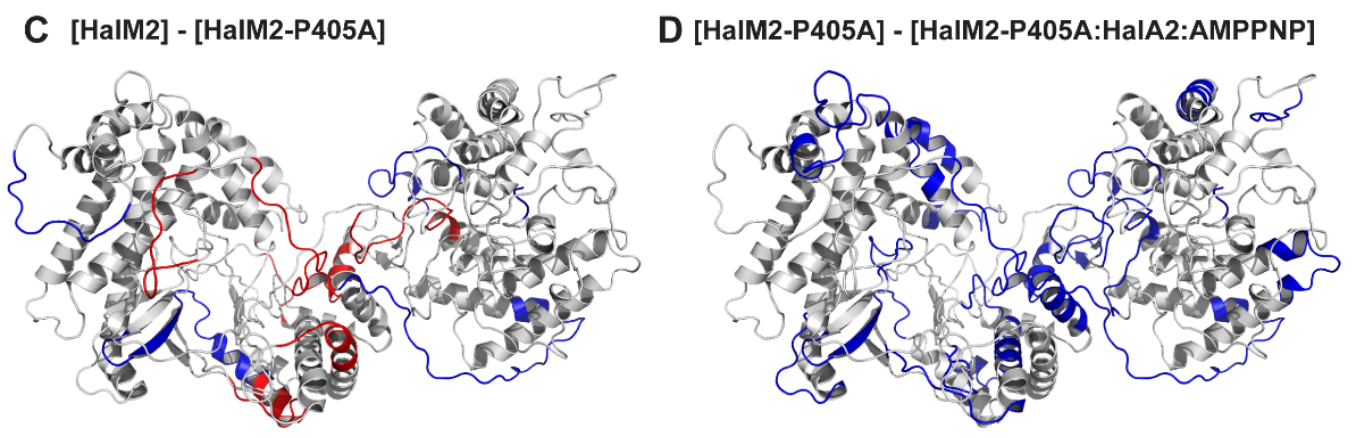

Figure S33. Deuterium uptake difference in peptides derived from HalM2 ${ }_{\mathrm{P} 405 \mathrm{~A}}$ in the presence and absence of AMP-PNP and HalA2 (panels $A$ and $D$ ) and relative to free wt HalM2 (panels $B$ and $C$ ). In (A) and (B), each bar represents a different HalM2P405Aderived peptide detected in our HDX-MS workflow. The HalM2 amino acid sequence and residue numbering is indicated in the coverage map. In the coverage maps (panels $A$ and $B$ ), the red and blue coloring indicate the uptake of more or less deuterium, respectively, upon ligand binding (panel A) or mutation (panel B). The peptides marked with a star exhibited a significant uptake difference (|uptake difference| $\geq 0.4 \mathrm{Da}$ ). The significant HDX peptides from $(A)$ and $(B)$ are mapped onto the HalM2 homology model in panels (D) and (C), respectively, using the same color scheme (red = more deuterium uptake, blue $=$ less deuterium uptake). A complete list of significant HDX peptides and their uptake difference values are provided in Table S5 and S6. 


\section{[HalM2T386A/D387A] - [HalM2T386A/D387A:HalA2:AMPPNP]}

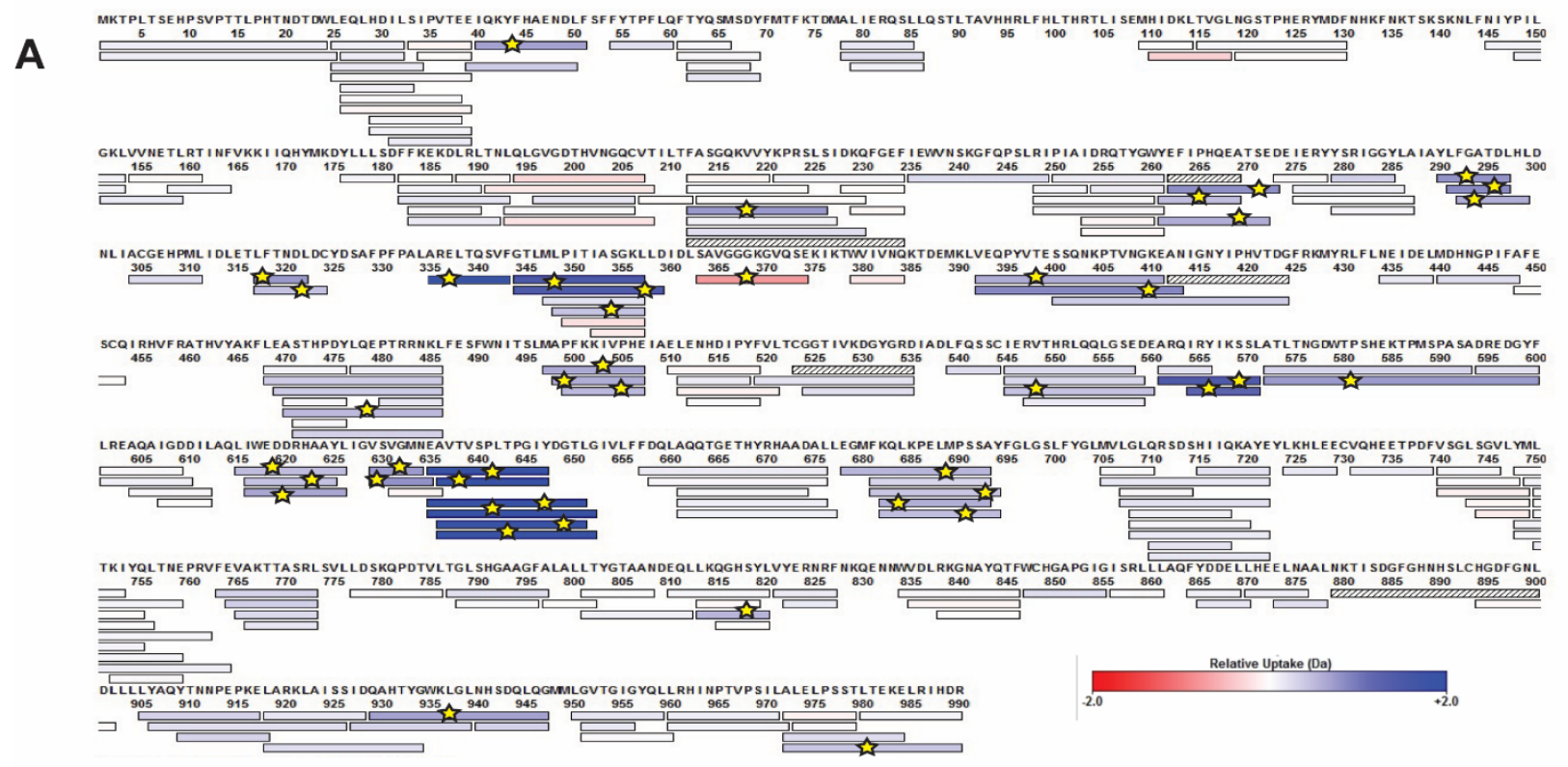

\section{[wt HalM2] - [HalM2T386A/D387A]}

B

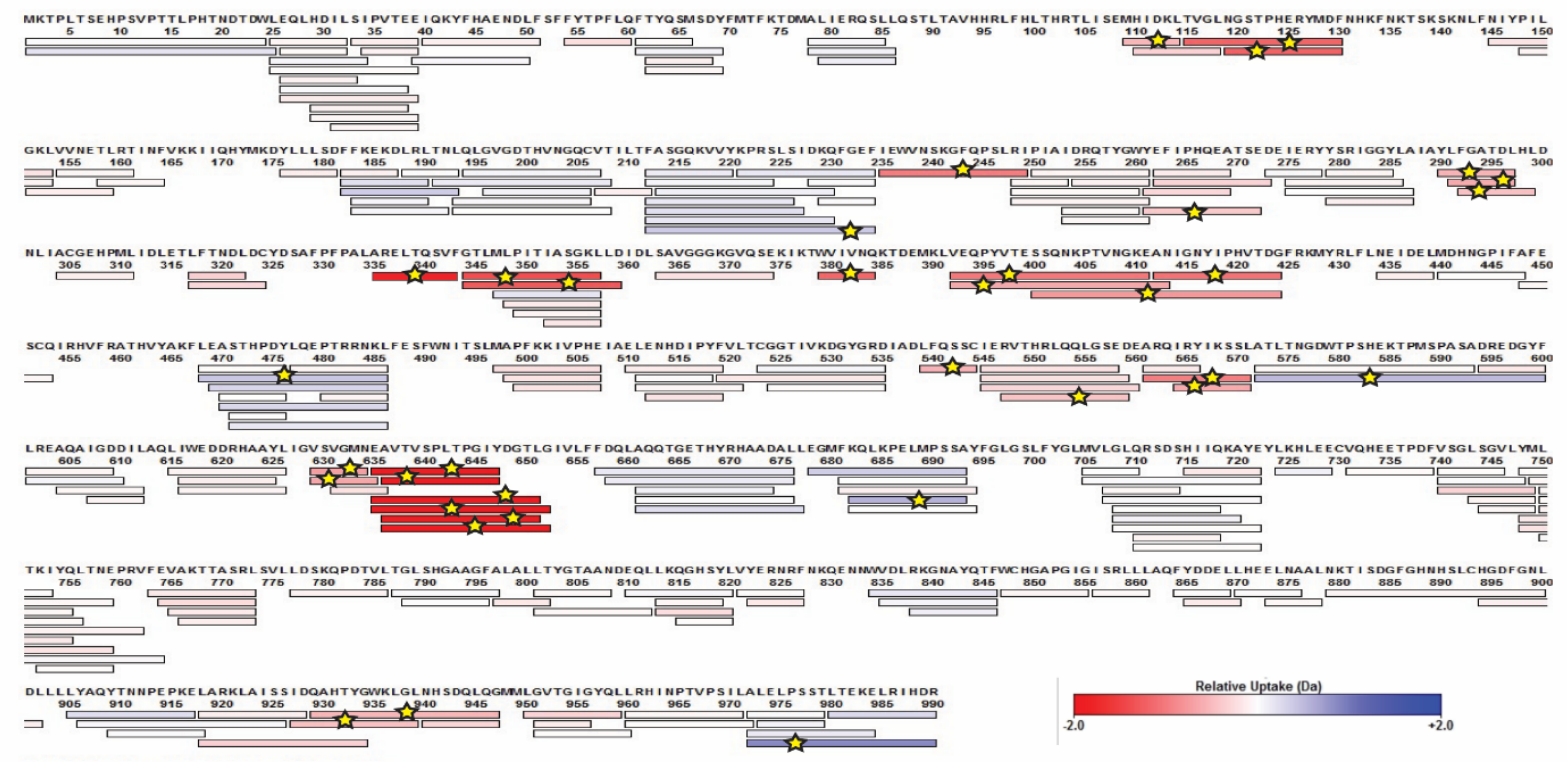



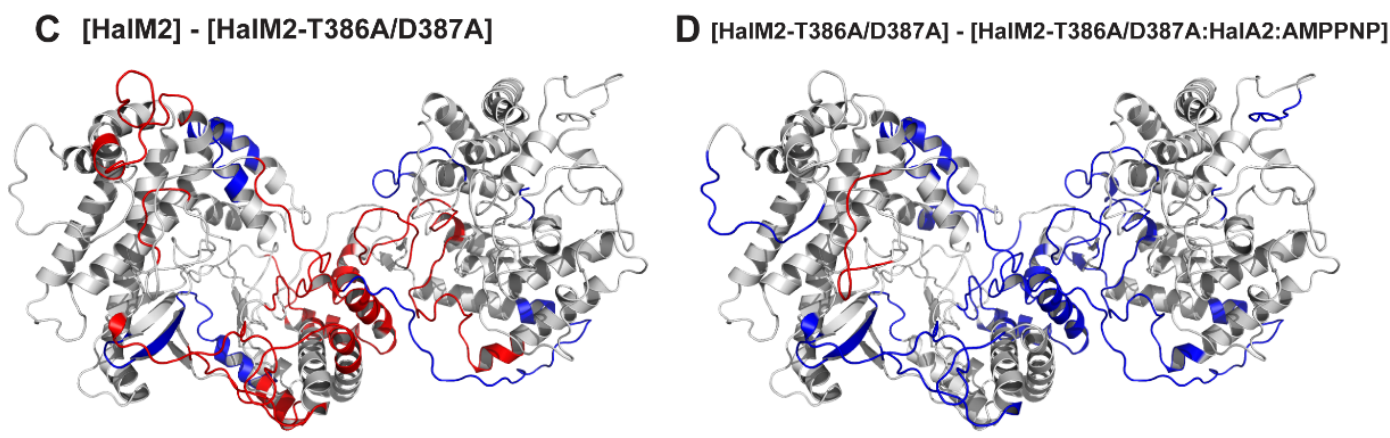

Figure S34. Deuterium uptake difference in peptides derived from HalM2T386A/D387A in the presence and absence of AMP-PNP and HalA2 (panels A and D) and relative to free wt HalM2 (panels B and C). In (A) and (B), each bar represents a different HalM2T386A/D387Aderived peptide detected in our HDX-MS workflow. The HalM2 amino acid sequence and residue numbering is indicated in the coverage map. In the coverage maps (panels $A$ and $B)$, the red and blue coloring indicate the uptake of more or less deuterium, respectively, upon ligand binding (panel A) or mutation (panel B). The peptides marked with a star exhibited a significant uptake difference (|uptake difference| $\geq 0.4 \mathrm{Da}$ ). The significant HDX peptides from (A) and (B) are mapped onto the HalM2 homology model in panels (D) and (C), respectively, using the same color scheme (red = more deuterium uptake, blue $=$ less deuterium uptake). A complete list of significant HDX peptides and their uptake difference values are provided in Table S5 and S6. 


\section{[HalM2 P349A] - [HalM2 P349A:HalA2:AMPPNP]}

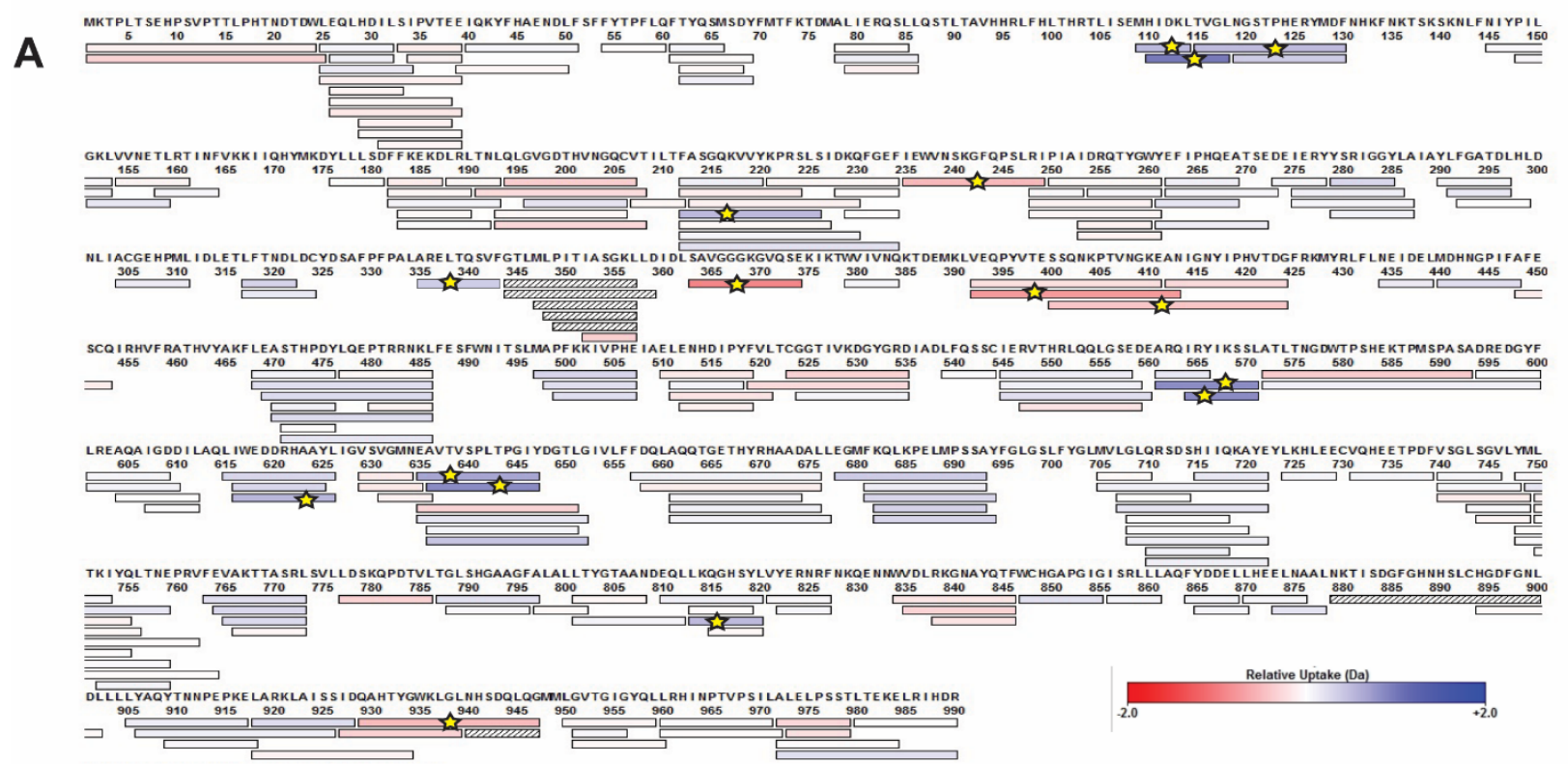

\section{[wt HalM2] - [HalM2 P349A]}

B

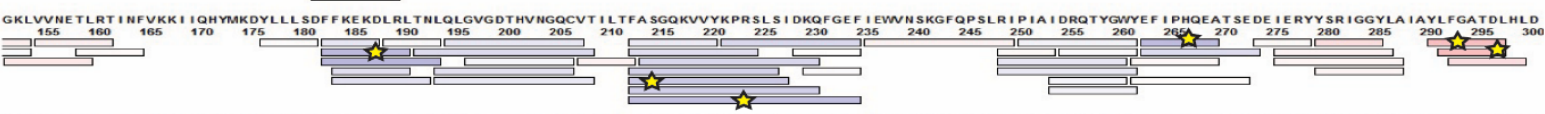

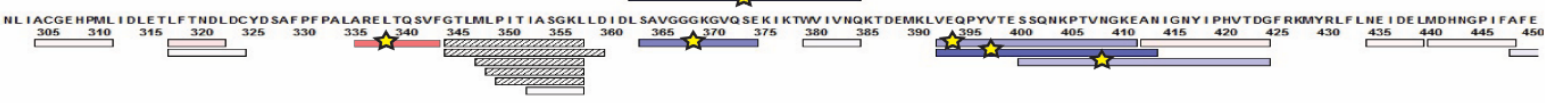

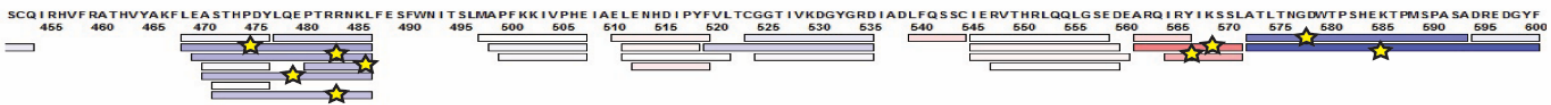

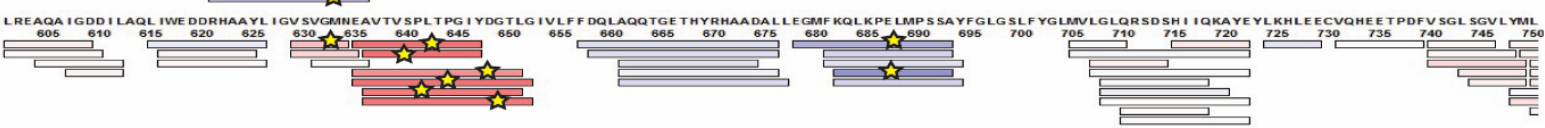

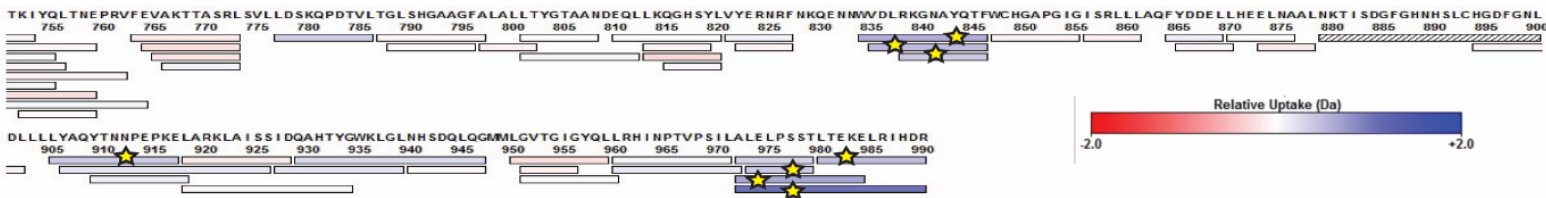


C [HalM2] - [HalM2-P349A]

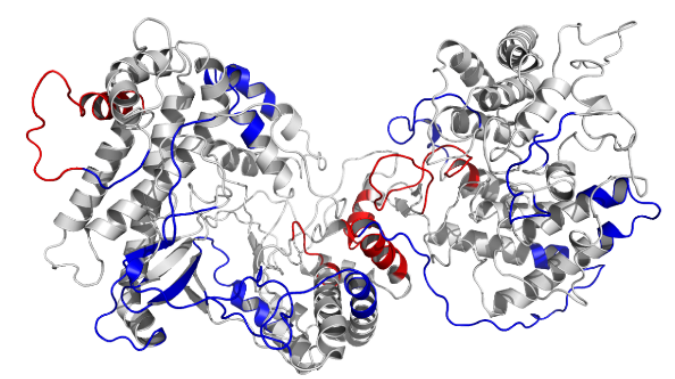

D [HalM2-P349A] - [HalM2-P349A:HaIA2:AMPPNP]

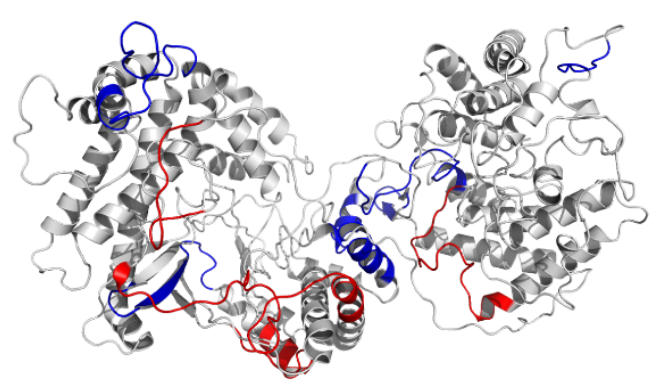

Figure S35. Deuterium uptake difference in peptides derived from HalM2 $2349 \mathrm{~A}$ in the presence and absence of AMP-PNP and HalA2 (panels $A$ and $D$ ) and relative to free wt HalM2 (panels $B$ and $C$ ). In (A) and (B), each bar represents a different HalM2P349Aderived peptide detected in our HDX-MS workflow. The HalM2 amino acid sequence and residue numbering is indicated in the coverage map. In the coverage maps (panels $A$ and $B)$, the red and blue coloring indicate the uptake of more or less deuterium, respectively, upon ligand binding (panel A) or mutation (panel B). The peptides marked with a star exhibited a significant uptake difference (|uptake difference| $\geq 0.4 \mathrm{Da}$ ). The significant HDX peptides from $(A)$ and $(B)$ are mapped onto the HalM2 homology model in panels (D) and (C), respectively, using the same color scheme (red = more deuterium uptake, blue $=$ less deuterium uptake). A complete list of significant HDX peptides and their uptake difference values are provided in Table S5 and S6. 


\section{[HalM2GS635-644] - [HalM2GS635-644:HalA2:AMPPNP]}

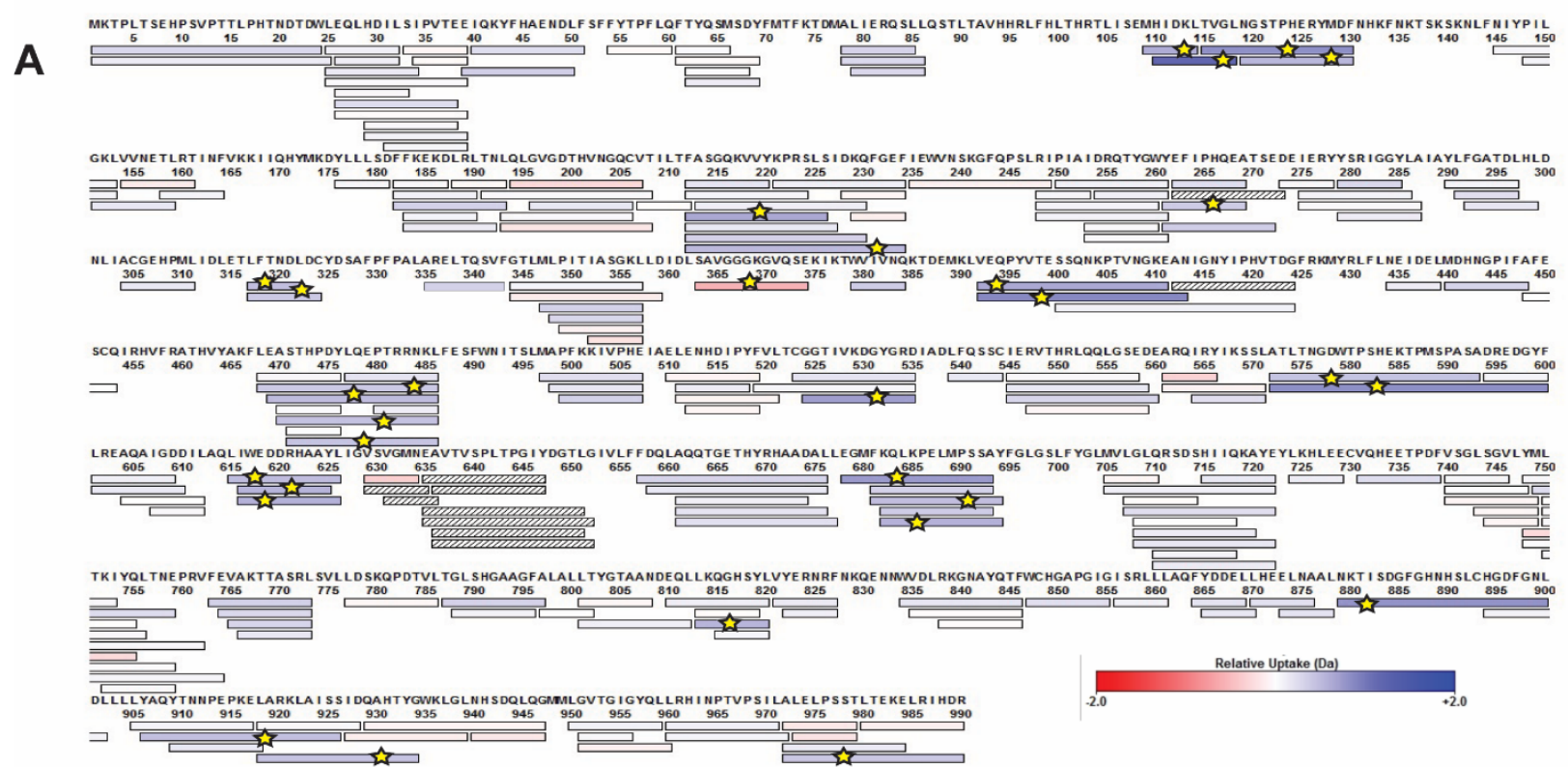

\section{[wt HalM2] - [HalM2GS635-644]}

B

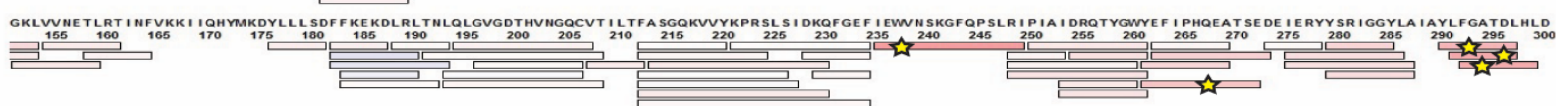

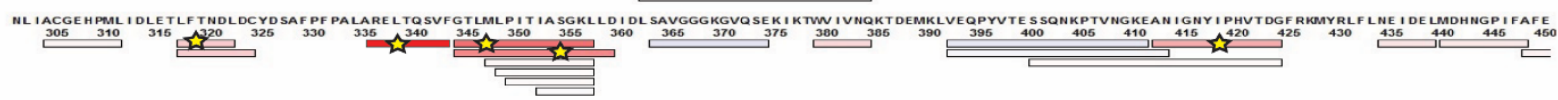

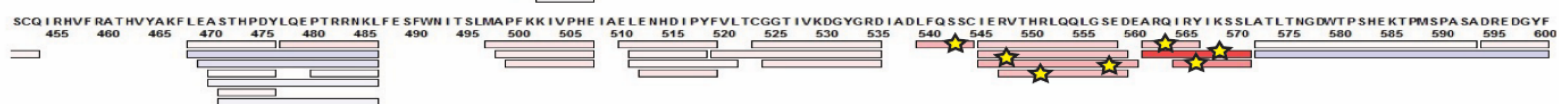

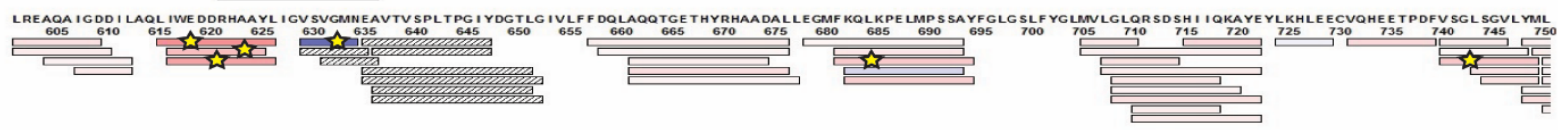

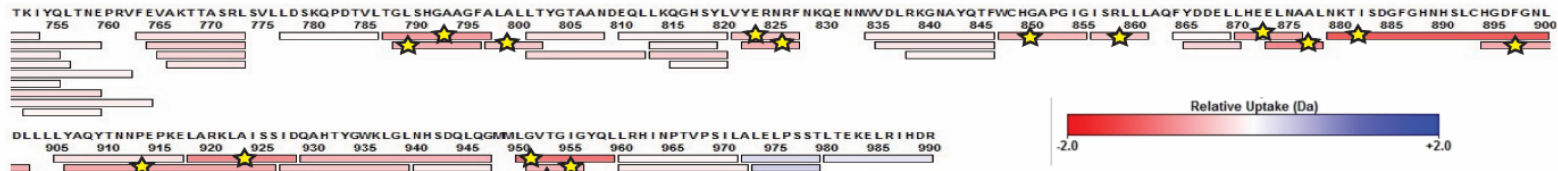


C [HalM2] - [HalM2-635-644]

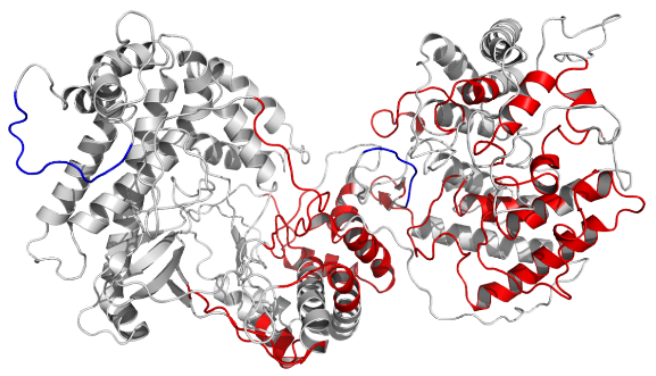

D [HalM2-635-644] - [HalM2-635-644:HalA2:AMPPNP]

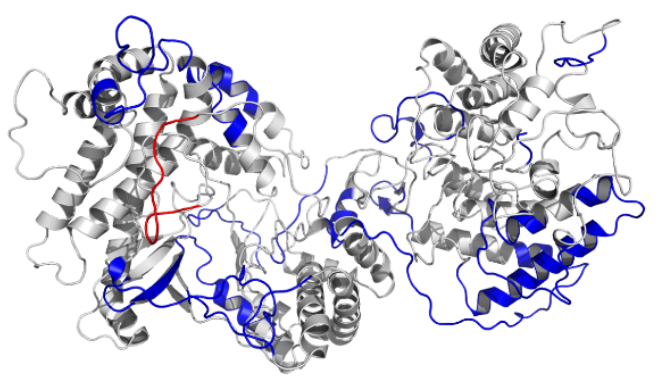

Figure S36. Deuterium uptake difference in peptides derived from HalM2GS635-644 in the presence and absence of AMP-PNP and HalA2 (panels A and D) and relative to free wt HalM2 (panels $B$ and $C$ ). In (A) and (B), each bar represents a different HalM2 ${ }_{G S 635-644-}$ derived peptide detected in our HDX-MS workflow. The HalM2 amino acid sequence and residue numbering is indicated in the coverage map. In the coverage maps (panels $A$ and $B)$, the red and blue coloring indicate the uptake of more or less deuterium, respectively, upon ligand binding (panel A) or mutation (panel B). The peptides marked with a star exhibited a significant uptake difference (|uptake difference| $\geq 0.4 \mathrm{Da}$ ). The significant HDX peptides from $(A)$ and $(B)$ are mapped onto the HalM2 homology model in panels (D) and (C), respectively, using the same color scheme (red = more deuterium uptake, blue $=$ less deuterium uptake). A complete list of significant HDX peptides and their uptake difference values are provided in Table S5 and S6. 


\section{[HalM2GS491-497] - [HalM2GS491-497:HalA2:AMPPNP]}

A

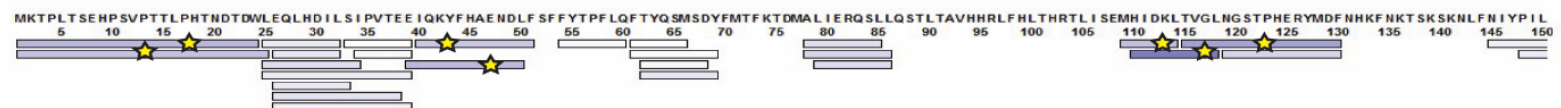
एह

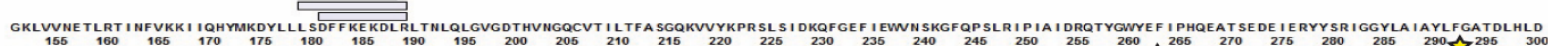
155

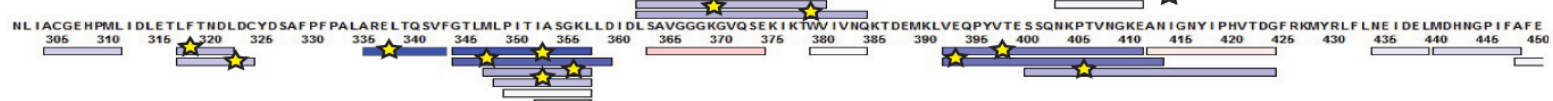

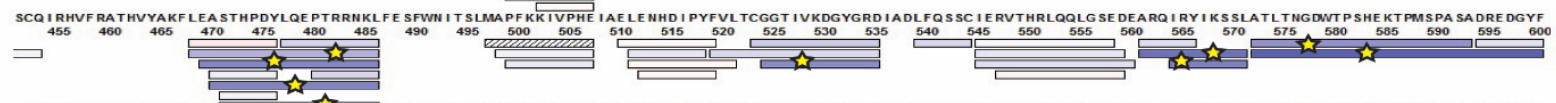

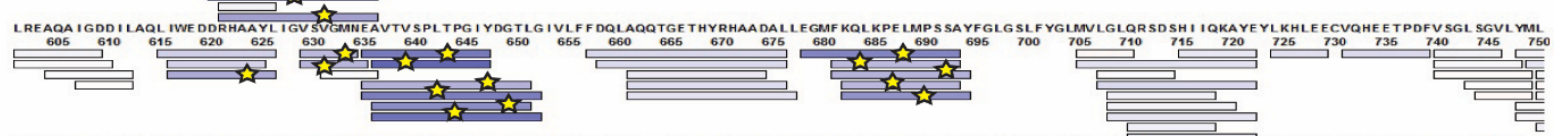

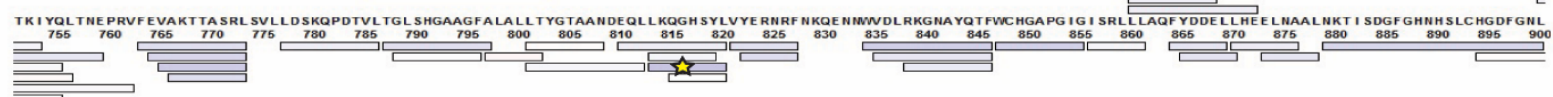

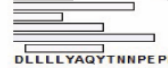

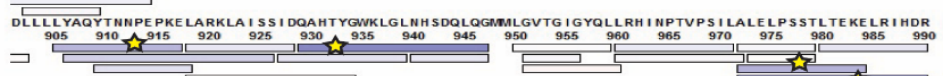

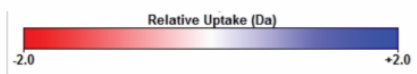

\section{[wt HalM2] - [HalM2GS491-497]}

B

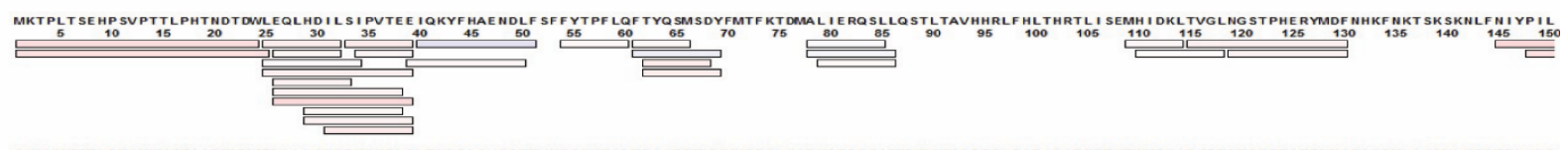

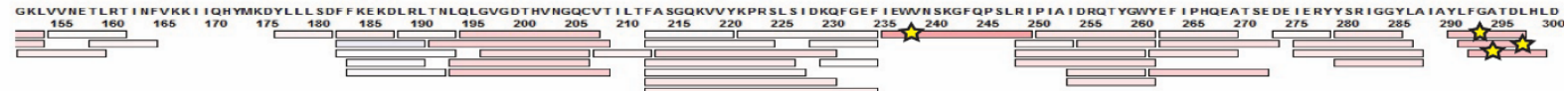

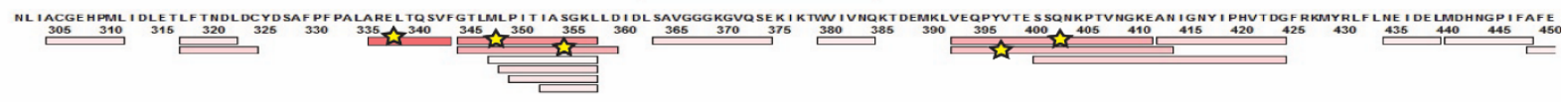

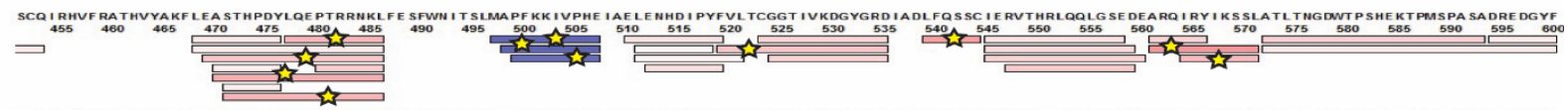

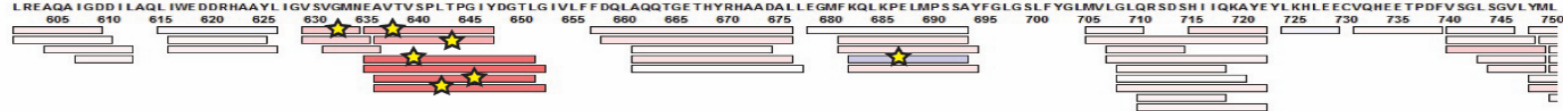

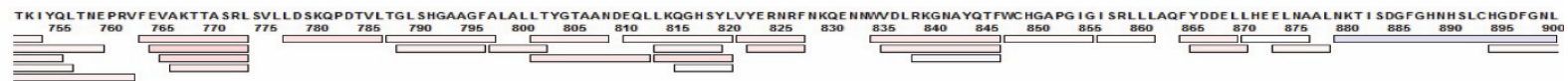

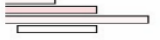

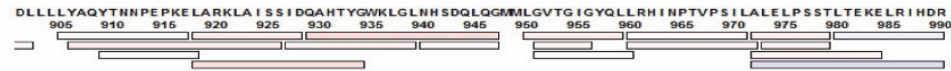

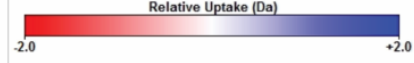



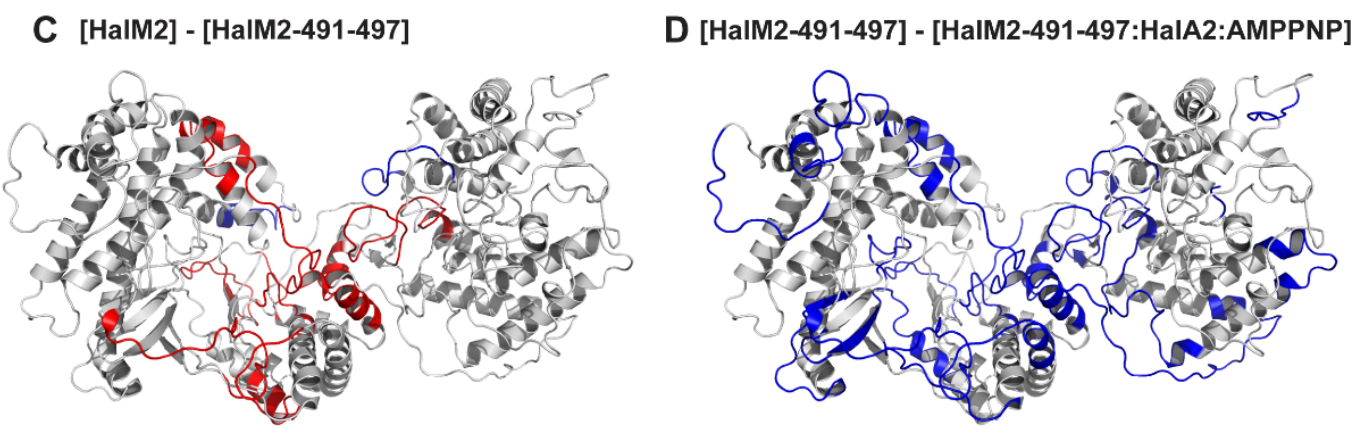

Figure S37. Deuterium uptake difference in peptides derived from HalM2GS491-497 in the presence and absence of AMP-PNP and HalA2 (panels A and D) and relative to free wt HalM2 (panels $B$ and $C$ ). In (A) and (B), each bar represents a different HalM2GS491-497derived peptide detected in our HDX-MS workflow. The HalM2 amino acid sequence and residue numbering is indicated in the coverage map. In the coverage maps (panels $A$ and $B)$, the red and blue coloring indicate the uptake of more or less deuterium, respectively, upon ligand binding (panel A) or mutation (panel B). The peptides marked with a star exhibited a significant uptake difference (|uptake difference| $\geq 0.4 \mathrm{Da}$ ). The significant HDX peptides from $(A)$ and $(B)$ are mapped onto the HalM2 homology model in panels (D) and (C), respectively, using the same color scheme (red = more deuterium uptake, blue $=$ less deuterium uptake). A complete list of significant HDX peptides and their uptake difference values are provided in Table S5 and S6. 


\section{[HalM2GS389-396] - [HalM2GS389-396:HalA2:AMPPNP]}

A

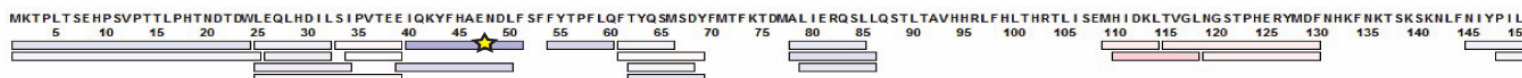

$$
\text { एक्य }
$$

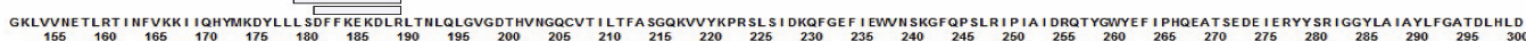
(150)

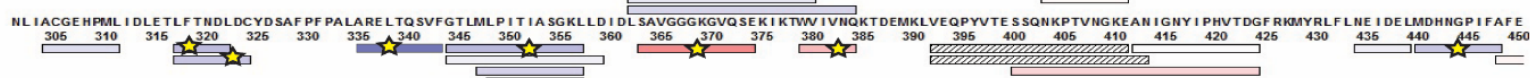

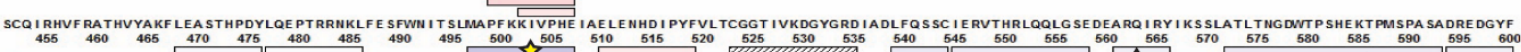
$\underbrace{455} \quad{ }^{460} \quad{ }_{465}$

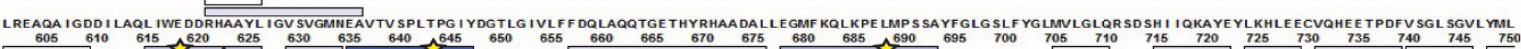

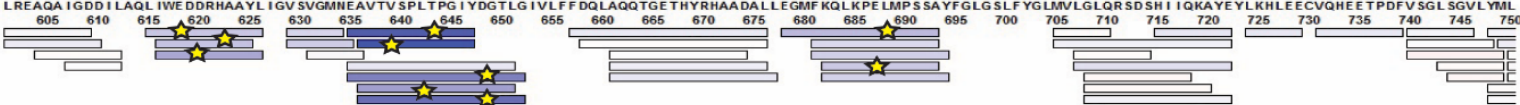

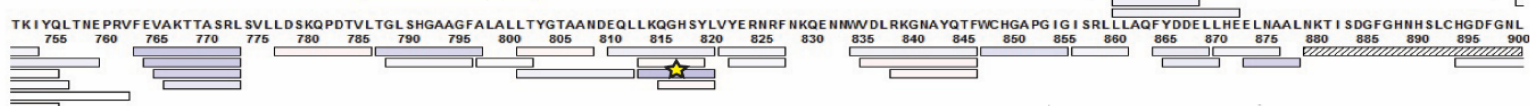

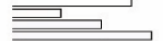

DLLLLYAYTTMNPE PKE LARKLAI IS I DQAHTYGWKLGLHH SDQLQGMMLGVTGIGYQLLRH I WPTVPS I LALELPS STLTE KELR I HDR

ב

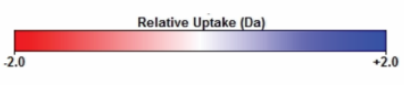

[wt HalM2] - [HalM2GS389-396]

B

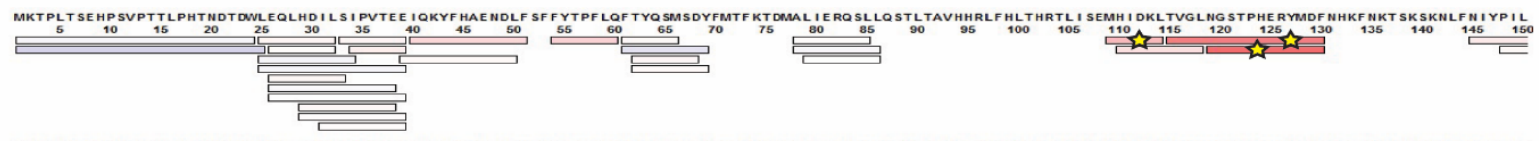

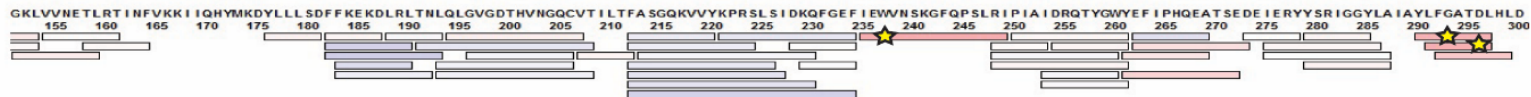

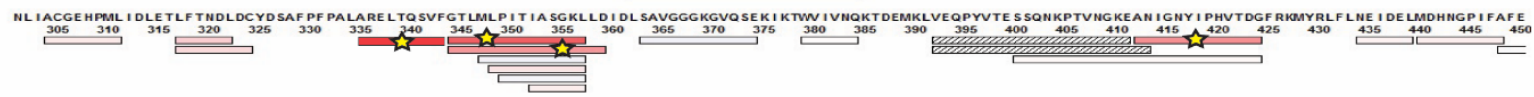

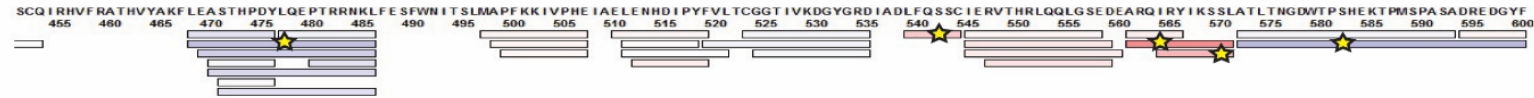

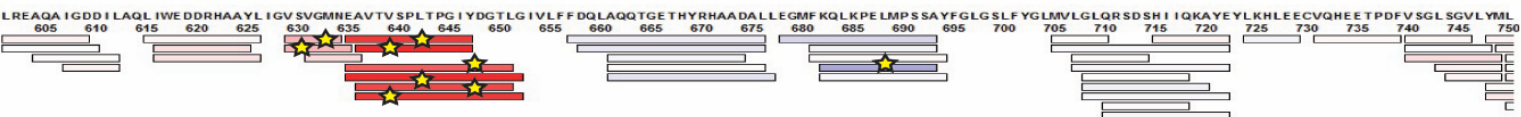

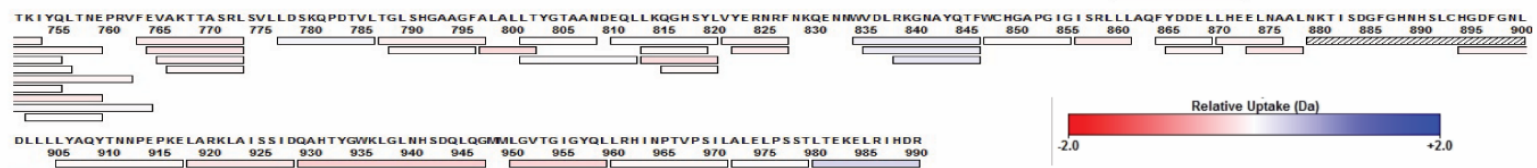


C [HalM2] - [HalM2-389-396]

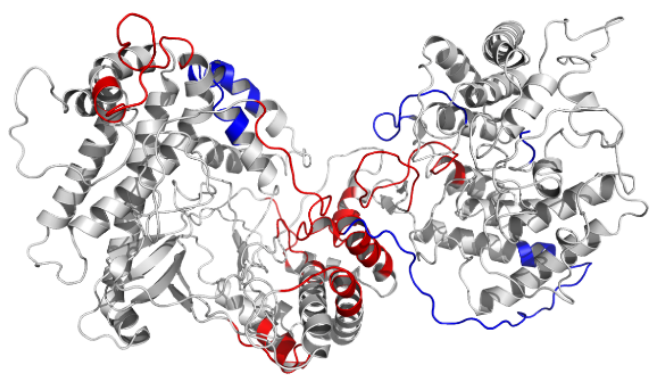

D [HalM2-389-396] - [HalM2-389-396:HalA2:AMPPNP]

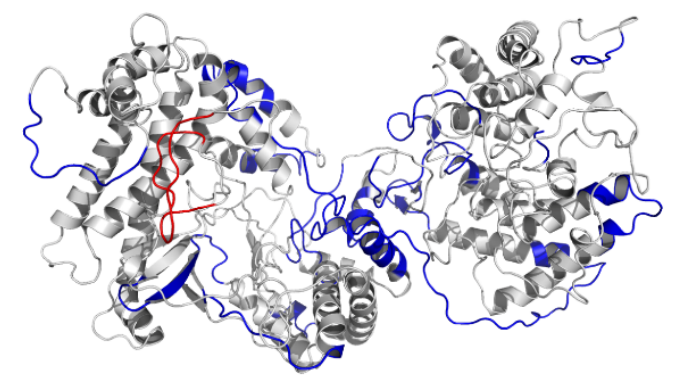

Figure S38. Deuterium uptake difference in peptides derived from HalM2GS389-396 in the presence and absence of AMP-PNP and HalA2 (panels A and D) and relative to free wt HalM2 (panels B and C). In (A) and (B), each bar represents a different HalM2GS389-396derived peptide detected in our HDX-MS workflow. The HalM2 amino acid sequence and residue numbering is indicated in the coverage map. In the coverage maps (panels $A$ and $B)$, the red and blue coloring indicate the uptake of more or less deuterium, respectively, upon ligand binding (panel A) or mutation (panel B). The peptides marked with a star exhibited a significant uptake difference (|uptake difference| $\geq 0.4 \mathrm{Da}$ ). The significant HDX peptides from $(A)$ and $(B)$ are mapped onto the HalM2 homology model in panels (D) and (C), respectively, using the same color scheme (red = more deuterium uptake, blue $=$ less deuterium uptake). A complete list of significant HDX peptides and their uptake difference values are provided in Table S5 and S6. 


\section{[HalM2GS360-365 ] - [HalM2GS360-365 :HalA2:AMPPNP]}

A ह

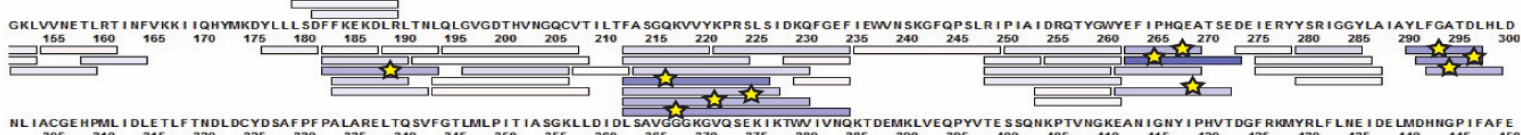

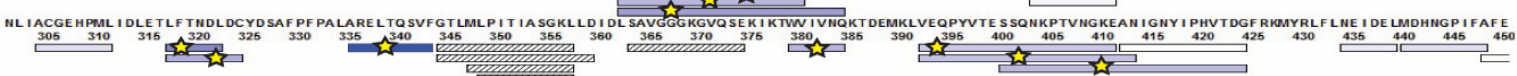

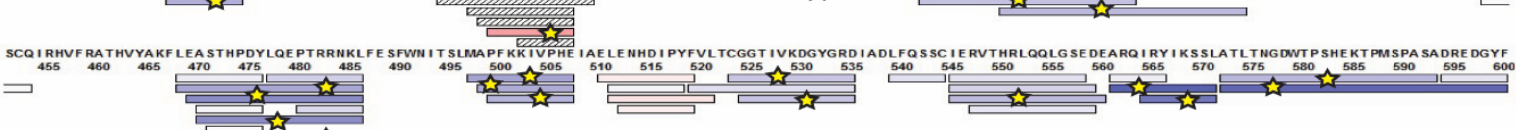

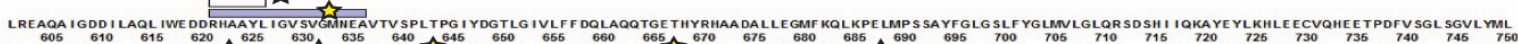
(150)

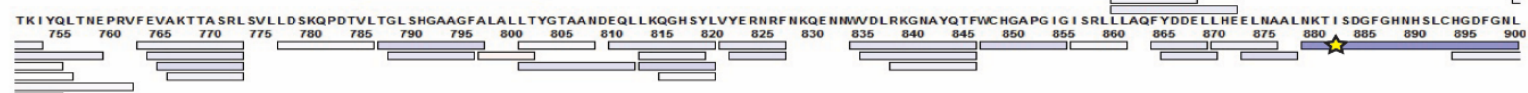

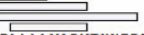

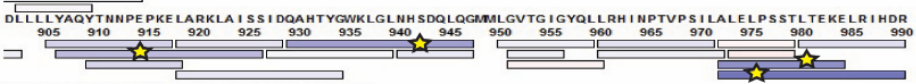

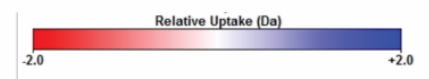

[wt HalM2] - [HalM2GS360-365]

B

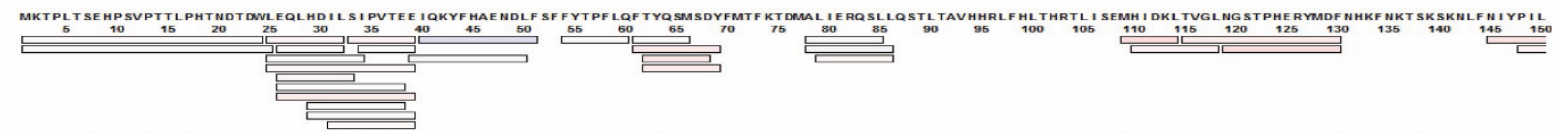

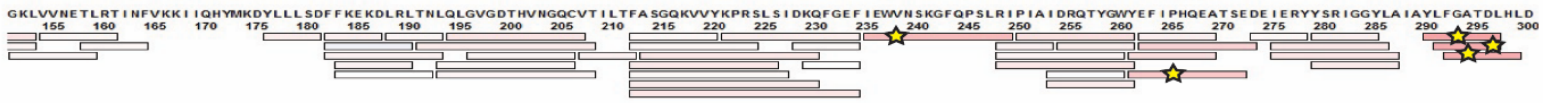

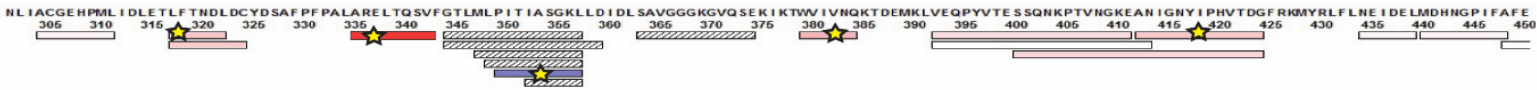

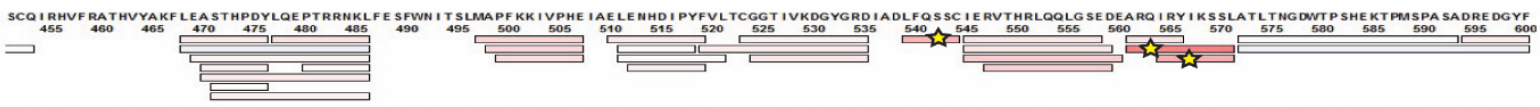

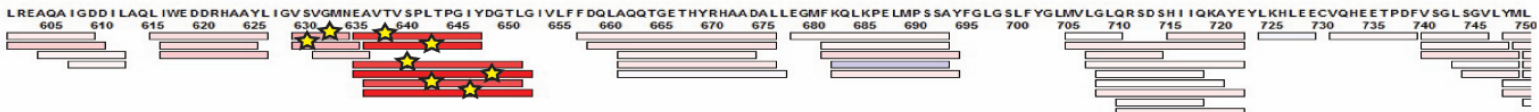

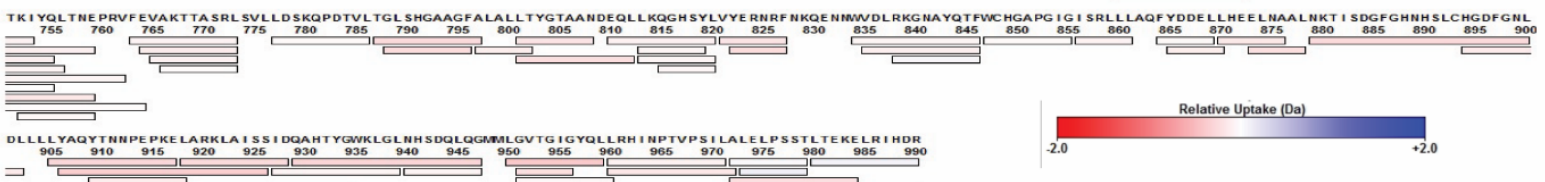


C [HalM2] - [HalM2-360-365]

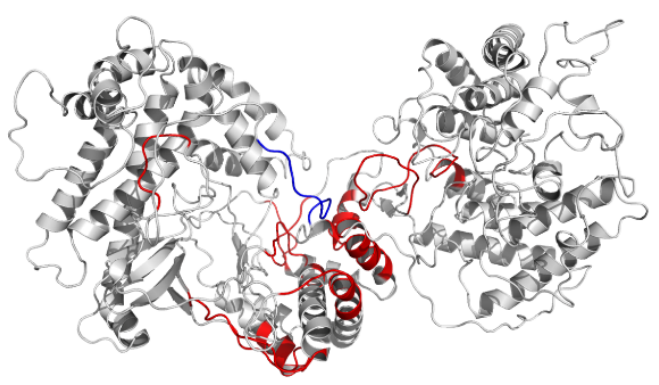

D [HalM2-360-365] - [HalM2-3360-365:HalA2:AMPPNP]

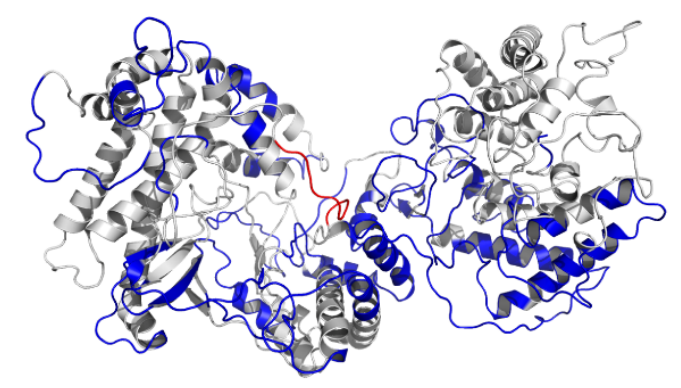

Figure S39. Deuterium uptake difference in peptides derived from HalM2 ${ }_{G S 360-365}$ in the presence and absence of AMP-PNP and HalA2 (panels A and D) and relative to free wt HalM2 (panels $B$ and $C$ ). In (A) and (B), each bar represents a different HalM2GS360-365derived peptide detected in our HDX-MS workflow. The HalM2 amino acid sequence and residue numbering is indicated in the coverage map. In the coverage maps (panels $A$ and $B$ ), the red and blue coloring indicate the uptake of more or less deuterium, respectively, upon ligand binding (panel A) or mutation (panel B). The peptides marked with a star exhibited a significant uptake difference (|uptake difference| $\geq 0.4 \mathrm{Da}$ ). The significant HDX peptides from (A) and (B) are mapped onto the HalM2 homology model in panels (D) and (C), respectively, using the same color scheme (red = more deuterium uptake, blue $=$ less deuterium uptake). A complete list of significant HDX peptides and their uptake difference values are provided in Table S5 and S6. 


\section{[HalM2GS331-340] - [HalM2GS331-340:HalA2:AMPPNP]}

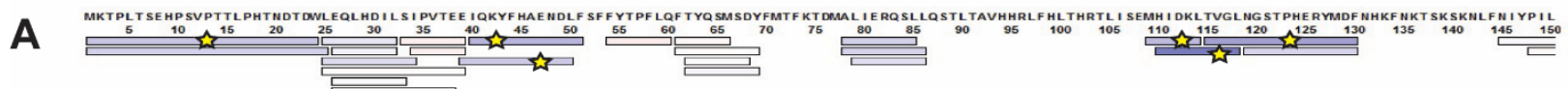
एव

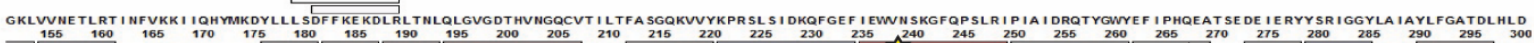
(150)

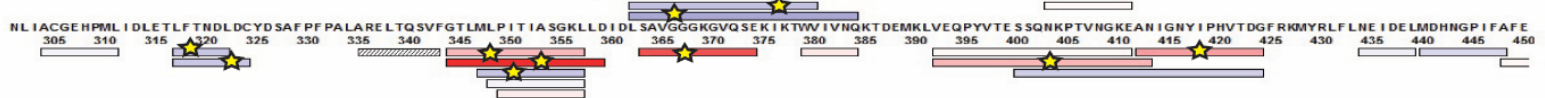

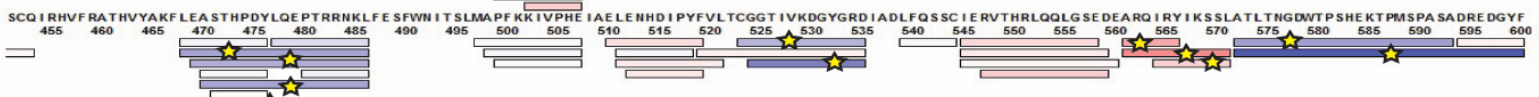

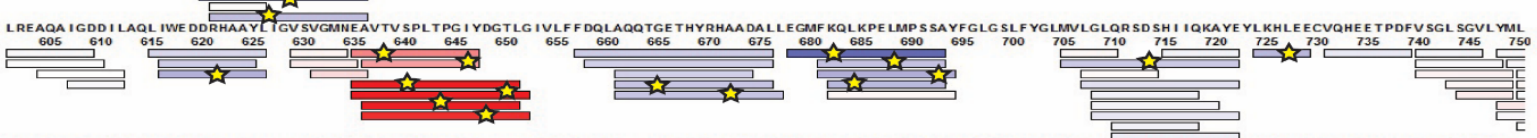

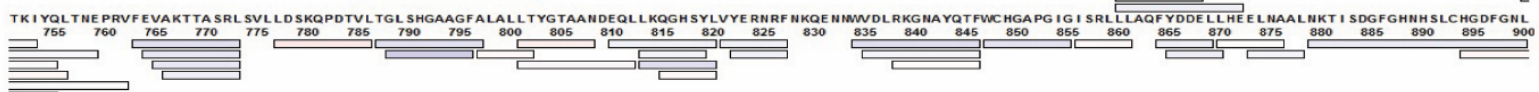

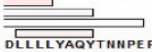

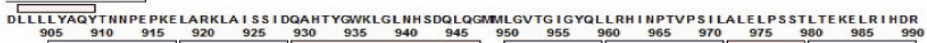

ב

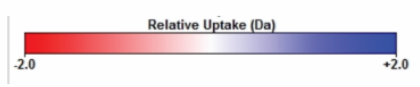

\section{[wt HalM2] - [HalM2GS331-340]}

B

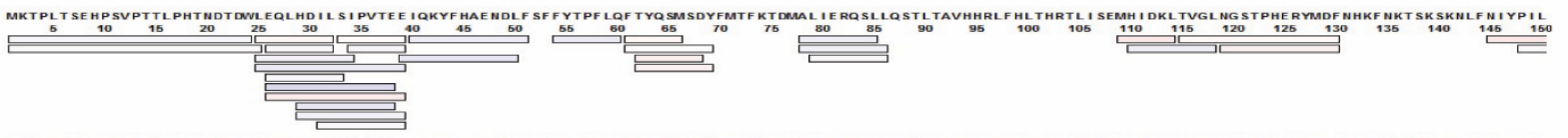

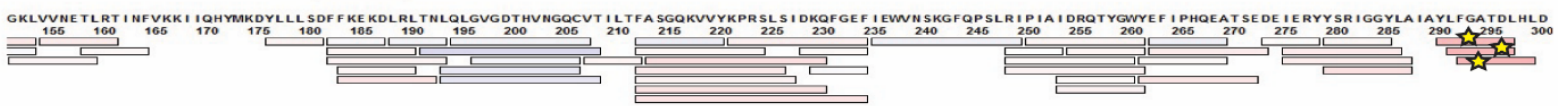

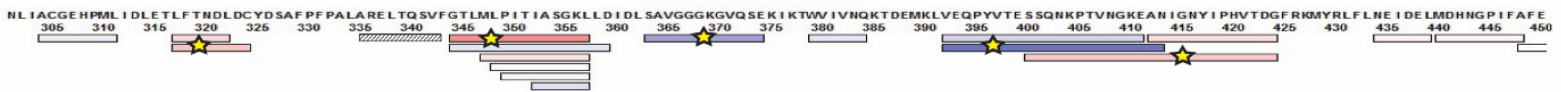

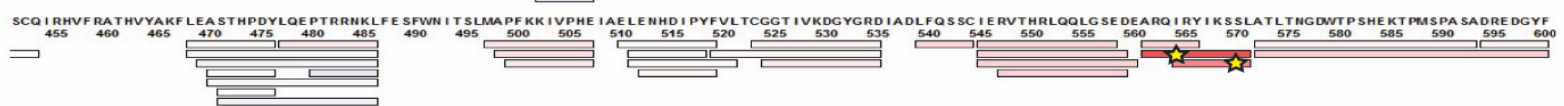

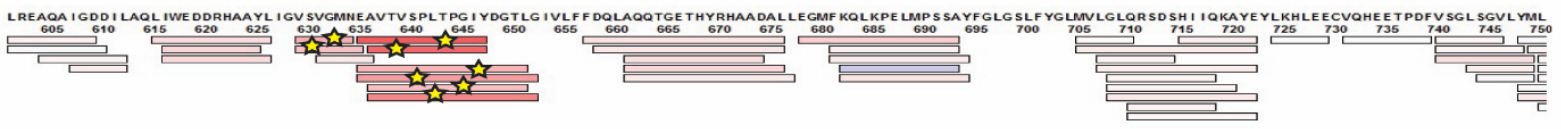

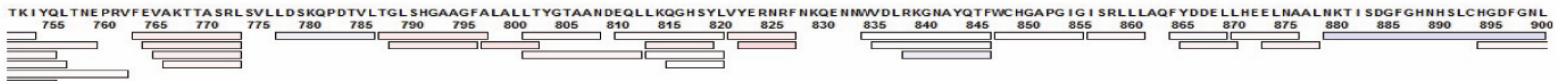
垔

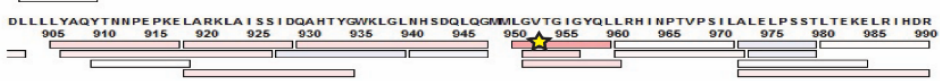

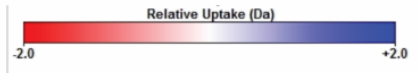



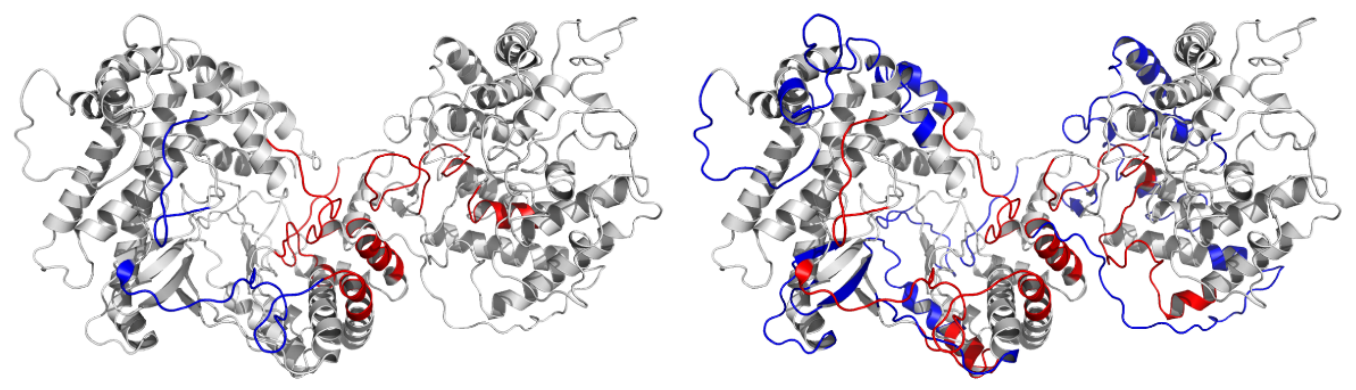

Figure S40. Deuterium uptake difference in peptides derived from HalM2GS331-340 in the presence and absence of AMP-PNP and HalA2 (panels A and D) and relative to free wt HalM2 (panels $B$ and $C$ ). In (A) and (B), each bar represents a different HalM2GS331-340derived peptide detected in our HDX-MS workflow. The HalM2 amino acid sequence and residue numbering is indicated in the coverage map. In the coverage maps (panels $A$ and $B)$, the red and blue coloring indicate the uptake of more or less deuterium, respectively, upon ligand binding (panel A) or mutation (panel B). The peptides marked with a star exhibited a significant uptake difference (|uptake difference| $\geq 0.4 \mathrm{Da}$ ). The significant HDX peptides from $(A)$ and $(B)$ are mapped onto the HalM2 homology model in panels (D) and (C), respectively, using the same color scheme (red = more deuterium uptake, blue $=$ less deuterium uptake). A complete list of significant HDX peptides and their uptake difference values are provided in Table S5 and S6. 


\section{[HalM2GS341-348 ] - [HalM2GS341-348 :HalA2:AMPPNP]}

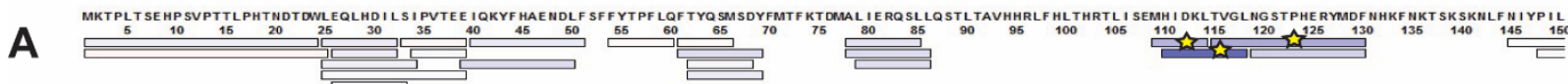
एह

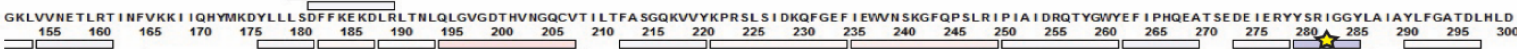

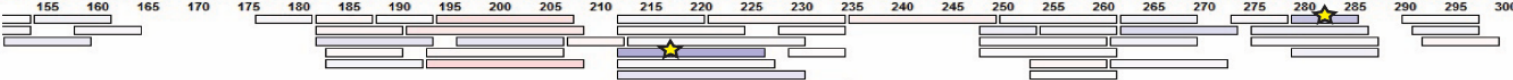

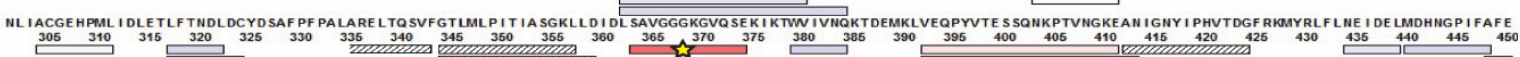

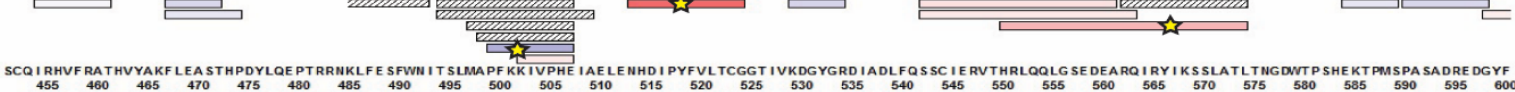

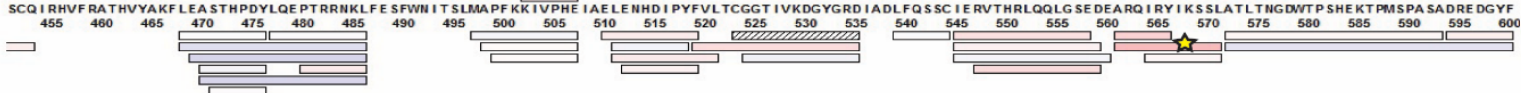

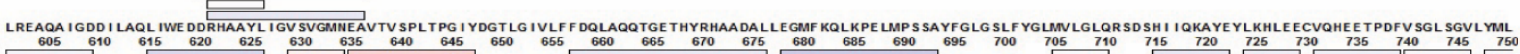
皮

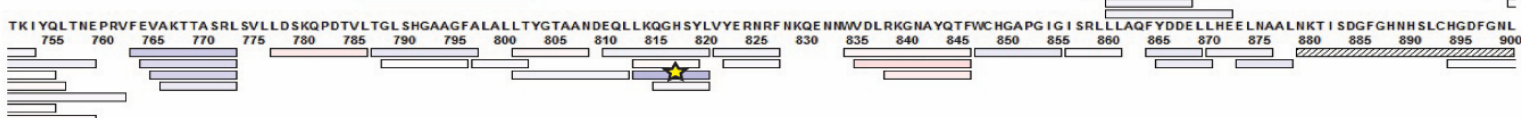

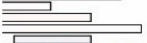

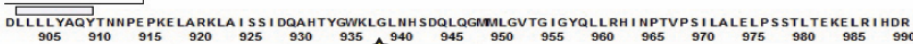
•

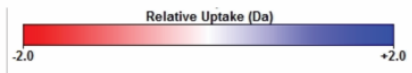

\section{[wt HalM2] - [HalM2GS341-348 ]}

B

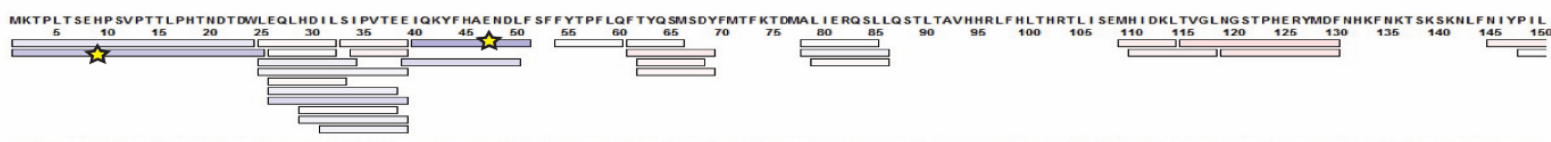

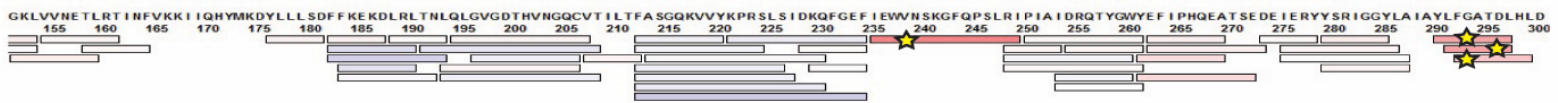

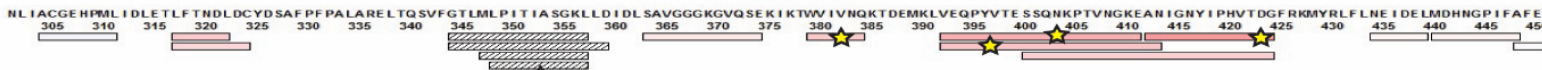
एin

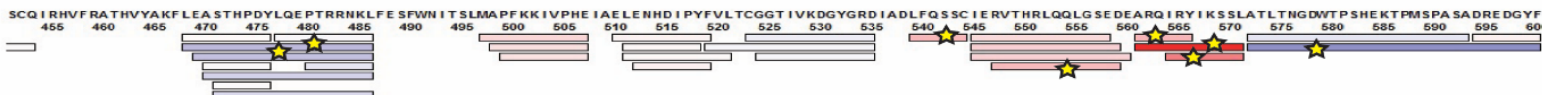

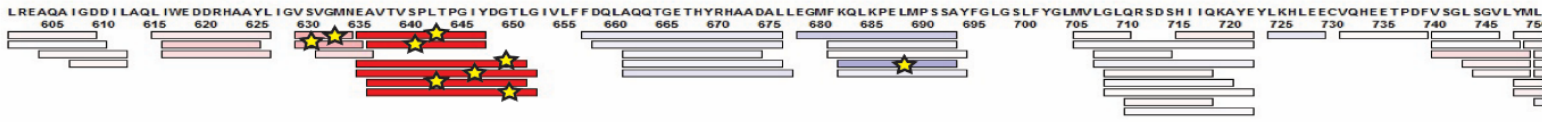

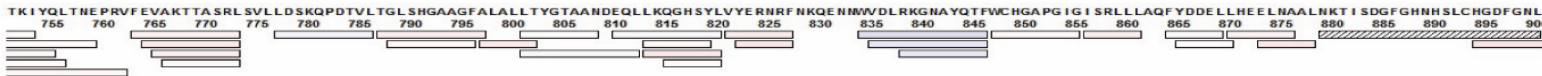
ב垔

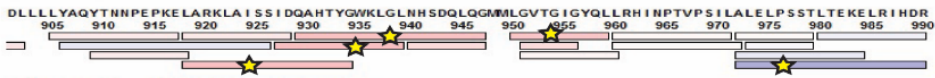

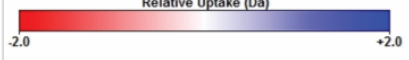


C

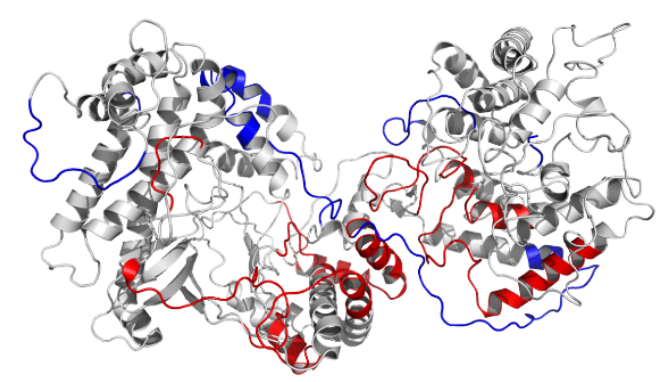

D [HalM2-341-348 ] - [HalM2-341-348:HalA2:AMPPNP]

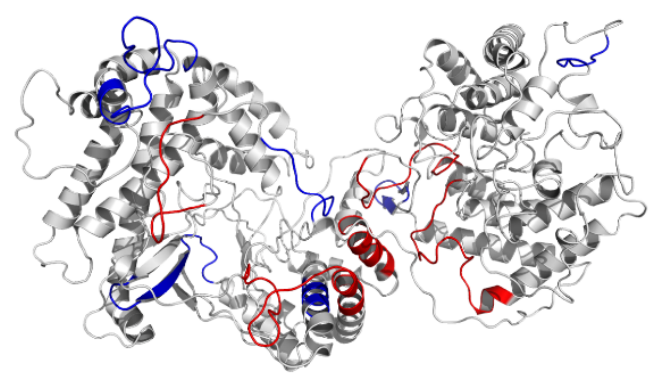

Figure S41. Deuterium uptake difference in peptides derived from HalM2GS341-348 in the presence and absence of AMP-PNP and HalA2 (panels $A$ and $D$ ) and relative to free wt HalM2 (panels B and C). In (A) and (B), each bar represents a different HalM2GS341-348derived peptide detected in our HDX-MS workflow. The HalM2 amino acid sequence and residue numbering is indicated in the coverage map. In the coverage maps (panels $A$ and $B$ ), the red and blue coloring indicate the uptake of more or less deuterium, respectively, upon ligand binding (panel A) or mutation (panel B). The peptides marked with a star exhibited a significant uptake difference (|uptake difference| $\geq 0.4 \mathrm{Da}$ ). The significant HDX peptides from $(A)$ and $(B)$ are mapped onto the HalM2 homology model in panels (D) and (C), respectively, using the same color scheme (red = more deuterium uptake, blue $=$ less deuterium uptake). A complete list of significant HDX peptides and their uptake difference values are provided in Table S5 and S6. 

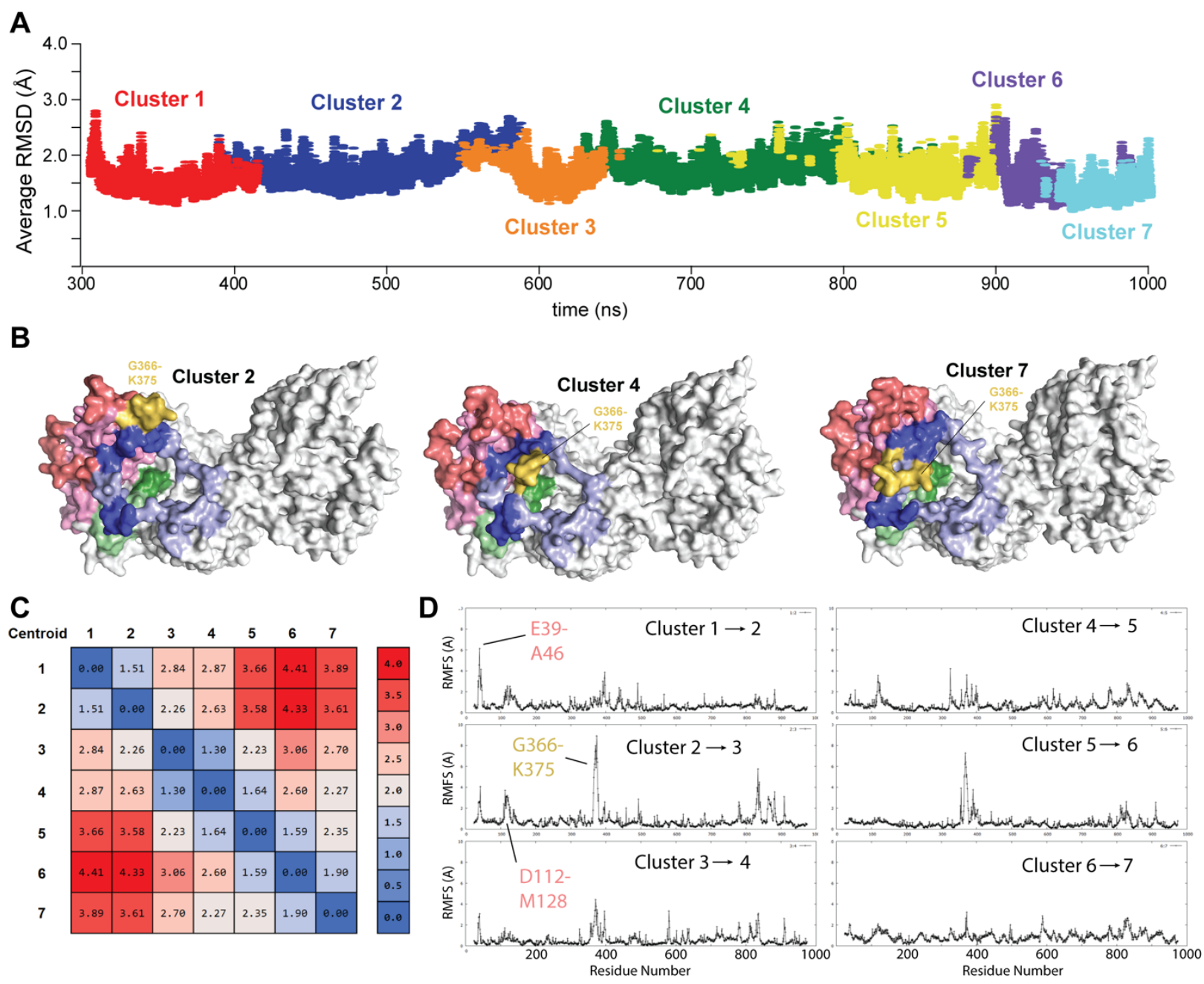

Figure S42. A) A cluster analysis of the HalM2 molecular dynamics simulation between $300-1000$ ns revealed 7 distinct, sequentially-formed HalM2 conformational populations. B) Representative structures from clusters 2, 4, and 7. A highly flexible glycine rich G366K375 segment (gold) of the P349-P405 loop can be seen in different conformations. The other regions of HalM2 are colored as in Figure 10 of the main text (pink tones - helical capping domain; blue tones - P349-P405 loop; green tones $-\mathrm{N}$ lobe $\beta$ sheet). C) Pairwise RMSD $(\AA)$ comparison between the representative centroid conformations of each HalM2 cluster. Red depicts greater conformational differences, while blue denotes smaller differences. The numbers on the top and left indicate the cluster number. D) A comparison of the RMSF of each HalM2 residue between pairs of adjacent clusters (indicated in each sub-panel). Note that most of the fluctuation between adjacent centroid 
conformations involves sections of HalM2 located in the N-terminal capping domain (HalM2 residues 40-50 and 110-130) and the P349-P405 loop. 


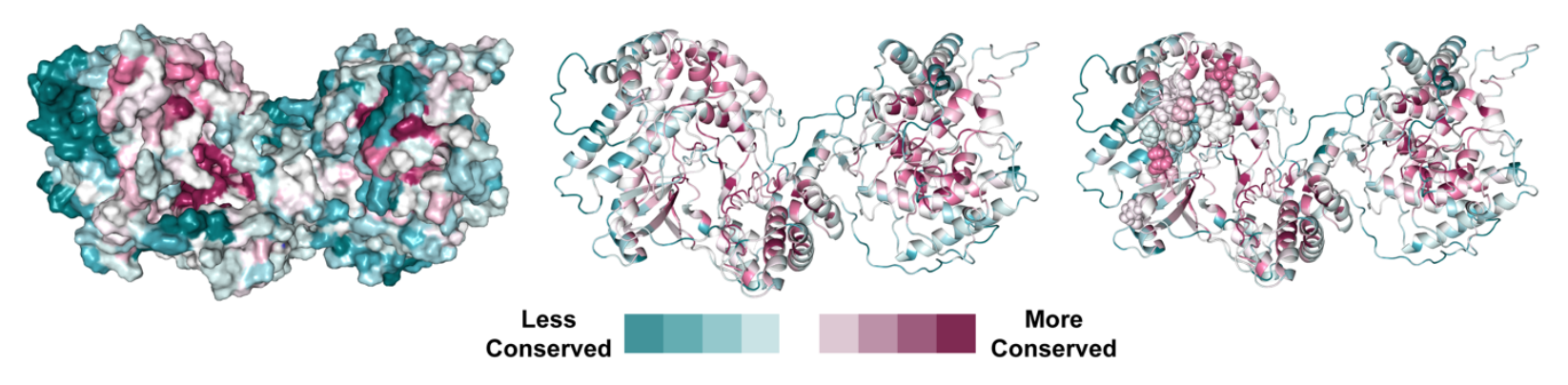

Figure S43. Relative levels of LanM amino acid conservation mapped onto the HalM2 homology model. The analysis was performed with the Consurf server using the MD simulation of the HalM2 homology model as the input structure and a manually constructed LanM multiple sequence alignment (available upon request). The structure is shown in the same orientation in each panel. The hydrophobic and polar contacts that form the interface between the P349-P405 loop, capping helices and N-lobe involve residues that are moderately to highly conserved. The side chains of these residues are shown in space filling representation in the structure on the right. 
Table S1. Oligonucleotide primer sequences used in this work.

\begin{tabular}{|c|c|c|c|}
\hline \# & Primer name & Sequence $\left(5^{\prime} \rightarrow 3^{\prime}\right)$ & $\begin{array}{l}\text { Paired primer for } \\
\text { Gibson Fragment } \\
\text { Amplification }\end{array}$ \\
\hline 1 & HalM2_P405A & CTTACGTAACCGAATCTAGCCAAAATAAAGCGACAGTTAACGGGAAA & 9 \\
\hline 2 & HalM2_P405A_rev & TTTCCCGTTAACTGTCGCTTTATTTTGGCTAGATTCGGTTACGTAAG & 10 \\
\hline 3 & HalM2_P349A & CTTTGGGACCTTGATGCTTGCGATTACAATTGCTAGTGGCA & 9 \\
\hline 4 & HalM2_P349A_rev & TGCCACTAGCAATTGTAATCGCAAGCATCAAGGTCCCAAAG & 10 \\
\hline 5 & HalM2_T386A/D387A & GATTAAAACATGGGTTATCGTCAATCAAAAAGCGGCTGAGATGAAGTTAGTT & 9 \\
\hline 6 & HalM2_T386A/D387A_rev & AACTAACTTCATCTCAGCCGCTTTTTGATTGACGATAACCCATGTTTTAATC & 10 \\
\hline 7 & HalM2_360-365_dgs3_FP & GGCTCTGGTTCCGGCTCTGGCGGGGGAAAAG & 9 \\
\hline 8 & HalM2_360-365_dgs3_RP & GTTAACGATCACCGTTTAATGAACTACCGAGACCAAGGCCAAGGCCTAGA & 10 \\
\hline 9 & pET15_Gib_FP & GTTGCCATTGCTGCAGGCATCGTGGTGTCAC & \\
\hline 10 & pET15_Gib_RP & GTGACACCACGATGCCTGCAGCAATGGCAAC & \\
\hline 11 & ProcM_FP_P460A & GTTGAAATTCCAACCAGTTTGGCAGTTGGAATTGGTGAGGTTAATC & 17 \\
\hline 12 & ProcM_RP_P460A & GATTAACCTCACCAATTCCAACTGCCAAACTGGTTGGAATTTCAAC & 18 \\
\hline 13 & ProcM_FP_S441A_D442A & CTTGGATTTAACGCCGCTGGAATGATGCCAG & 17 \\
\hline 14 & ProcM_RP_S441A_D442A & CTGGCATCATTCCAGCGGCGTTAAATCCAAG & 18 \\
\hline 15 & ProcM_FP_P404A & CGTTCCGGTTTACTCGCGCAATGGATGTTTCTTG & 17 \\
\hline 16 & ProcM_RP_P404A & CAAGAAACATCCATTGCGCGAGTAAACCGGAAC & 18 \\
\hline 17 & ProcM_pET28_Gib_FP & GCGATCCCCGGGAAAACAGCATTCCAGG & \\
\hline 18 & ProcM_pET28_Gib_RP & CCTGGAATGCTGTTTTCCCGGGGATCGC & \\
\hline 19 & ProcM_notag_prsf_FP & GTATATTAGTTAAGTATAAGAAGGAGATATACATATGGAAAGTCCATCATCTTGG & 20 \\
\hline 20 & ProcM_notag_prsf_RP & GGCCGGCCGATATCCAATTGAGATCTGCCATTTATTCAGTAGGCCAGAGACCAG & 19 \\
\hline 21 & ProcA2.8_histag_prsf_FP & CACCATCATCACCACAGCCAGGATCCGATGTCAGAAGAGCAACTGAAGGC & 22 \\
\hline 22 & ProcA2.8_histag_prsf_RP & CGACCTGCAGGCGCGCCGAGCTCGAATTTTAGCACTCACCCTCCCAATAG & 21 \\
\hline 23 & ProcM_Gib_FP & GCGGCCTGGTGCCGCGCGGCAGCCATATGGAAAGTCCATCATCTTGG & 24 \\
\hline 24 & ProcM_Gib_RP & TGCTGTCCACCAGTCATGCTAGCCATATTATTCAGTAGGCCAGAGACCAG & 23 \\
\hline 25 & ProcA2.8_Gib_FP & GCGGCCTGGTGCCGCGCGGCAGCCATATGTCAGAAGAGCAACTGAAGG & 26 \\
\hline 26 & ProcA2.8_Gib_RP & TGCTGTCCACCAGTCATGCTAGCCATATTAGCACTCACCCTCCCAATAG & 25 \\
\hline
\end{tabular}

Table 4.1: List of oligonucleotide primers used in this work 
Table S2. Simulation of wt HalM2 kinetic data to previously reported model. ${ }^{2}$

\begin{tabular}{|c|c|c|c|c|c|}
\hline & \multicolumn{2}{|c|}{ Previous Simulation } & \multicolumn{2}{|c|}{ New Simulation } & \multirow{2}{*}{$\begin{array}{c}\text { Ratio V/K New } \\
\text { vs Old fit }\end{array}$} \\
\hline Step & Rates & $V / K$ & Rates & $V / K$ & \\
\hline$k_{o n}$ & 10.0 & & $13.5^{e}$ & & \\
\hline$k_{\text {off }}$ & 18.0 & & $24.3^{e}$ & & \\
\hline$k_{0,0 \rightarrow 0,1}$ & $17.0(15.1-18.8)$ & 4.9 & $75.6(70.0-81.2)^{e}$ & 10.2 & 2.1 \\
\hline$k_{0,1 \rightarrow 0,2}$ & $61.0(57.1-70.2)$ & 7.7 & $149(135-167)$ & 11.6 & 1.5 \\
\hline$k_{0,2 \rightarrow 0,3}$ & $25.0(15.7-38.4)$ & 5.8 & $77.3(61.8-106)$ & 10.2 & 1.8 \\
\hline$k_{0,2 \rightarrow 1,2}$ & $104(90.7-117)^{a}$ & 8.5 & $143(128-163)^{a}$ & 11.5 & 1.4 \\
\hline$k_{0,3 \rightarrow 1,3}$ & $104^{a}$ & 8.5 & $143^{a}$ & 11.5 & 1.4 \\
\hline$k_{1,2 \rightarrow 1,3}$ & $81.0(70.3-89.1)$ & 8.2 & $230(184-288)^{d}$ & 12.2 & 1.5 \\
\hline$k_{1,3 \rightarrow 1,4}$ & $36.0(32.9-40.3)$ & 6.7 & $121^{d}$ & 11.2 & 1.7 \\
\hline$k_{1,4 \rightarrow 1,5}$ & $16.0(13.9-17.6)$ & 4.7 & $44(35.2-55)$ & 8.7 & 1.8 \\
\hline$k_{1,5 \rightarrow 1,6}$ & $23.0(18.5-28.9)$ & 5.6 & $68.3(61.3-83.6)$ & 10.0 & 1.8 \\
\hline$k_{1,4 \rightarrow 2,4}$ & $2.5(1.9-3.2)^{b}$ & 1.2 & $3.79(3.0-4.8)^{b}$ & 1.8 & 1.5 \\
\hline$k_{1,5 \rightarrow 2,5}$ & $5.0^{b}$ & 2.2 & $6.08^{b}$ & 2.7 & 1.2 \\
\hline$k_{1,6 \rightarrow 2,6}$ & $20.0(16.1-25.2)$ & 5.3 & $52.7(46.4-60.3)$ & 9.23 & 1.8 \\
\hline$k_{2,4 \rightarrow 2,5}$ & $19.0(12.0-29.3)$ & 5.1 & $26.1(16.7-43.4)$ & 7.0 & 1.4 \\
\hline$k_{2,5 \rightarrow 2,6}$ & $32.0(26.6-39.5)^{c}$ & 6.4 & $47.1(42.0-53.5)^{c}$ & 8.9 & 1.4 \\
\hline$k_{2,6 \rightarrow 2,7}$ & $58.0^{c}$ & 7.6 & $148^{c}$ & 11.6 & 1.5 \\
\hline$k_{2,7 \rightarrow 4,7}$ & $41.0^{c}$ & 6.9 & $103^{c}$ & 10.9 & 1.6 \\
\hline$\chi^{2} / D O F$ & 1.12 & & 3.70 & & \\
\hline Avg & & & & & 1.6 \\
\hline Std Dev & & & & & 0.23 \\
\hline Std Error & & & & & 0.06 \\
\hline
\end{tabular}

${ }^{*} k_{\text {on }}$ is reported in units of $\mu \mathrm{M}^{-1} \mathrm{~min}^{-1}$. All other apparent rate constants and their corresponding FitSpace boundaries (shown in parentheses) are reported in units of $\operatorname{~min}^{-1}$. ${ }^{a-e}$ Indicates rates that were linked at a fixed ratio and were varied as single parameter in the global fit. The catalytic efficiency $(V / K)$ is in units of $\mu \mathrm{M}^{-1} \mathrm{~min}^{-1}$ and was calculated as: $V / K=\left(k_{o n} k_{x}\right) /\left(k_{o f f}+k_{x}\right)$, where $k_{x}$ is the rate constant for the chemistry step in question. The ratio of $V / K$ determined from the present data and the data reported in ref 2 show that there is a systematic 1.6 -fold increase in $\mathrm{V} / \mathrm{K}$ determined from the present data. This may be due to the quality/activity of the enzyme preparation and or to slight differences in the peptide concentrations used in the assays. Nevertheless, the fact that this ratio is approximately constant for all of the chemical transformations suggests that the relative ratios between the kinetic parameters are very reproducible. 
Table S3. Comparison of cyclization models 1-3.

\begin{tabular}{|c|c|c|c|c|c|c|c|}
\hline & & wt HalM2 & HalM2P405A & HalM2 ${ }_{T 386 A / D 387 A}$ & HalM2P349A & HalM2 ${ }_{G S 635-644}$ & HalM2 $2_{G S 491-497}$ \\
\hline \multirow{11}{*}{$\begin{array}{c}\text { Model } \\
1\end{array}$} & $k_{\text {on }}$ & $24.5(22.0-26.9)^{a}$ & $8.0(7.4-8.6)^{a}$ & $4.6(4.1-5.3)^{a}$ & $4.2(3.6-4.9)^{a}$ & $19.1(18.0-20.4)^{a}$ & $18.9(17.9-20.8)^{a}$ \\
\hline & $k_{\text {off }}$ & $20.0^{a}$ & $19.5^{a}$ & $18.5^{a}$ & $9.4^{a}$ & $16.9^{a}$ & $71.9^{a}$ \\
\hline & $k_{c, 0 \rightarrow 1}$ & $66.1^{a}$ & $8.9^{a}$ & $6.4^{a}$ & $1.1^{a}$ & $3.5^{a}$ & $1.9^{a}$ \\
\hline & $k_{c, 1 \rightarrow 2}$ & $13.9(12.5-15.5)$ & $7.1(6.3-7.8)$ & $8.0(7.0-9.3)$ & $1.4(1.1-1.9)$ & $1.2(1.0-1.3)$ & $1.9(1.6-2.1)$ \\
\hline & $k_{c, 2 \rightarrow 3}$ & $38.9(34.2-44.8)^{b}$ & $23.3(21.2-29.1)^{b}$ & $13.9(11.2-17.4)^{b}$ & $2.6(2.2-3.2)^{b}$ & $1.8(1.6-2.1)$ & $5.4(4.7-6.7)^{b}$ \\
\hline & $k_{c, 3 \rightarrow 4}$ & $391^{b}$ & $233^{b}$ & $140^{b}$ & $25.7^{b}$ & $2.0(1.7-2.3)$ & $2.3^{b}$ \\
\hline & $k_{c, 1 \rightarrow 0}$ & & & & & $0.39(0.31-0.49)^{b}$ & \\
\hline & $k_{c, 2 \rightarrow 1}$ & & & & & $0.77^{b}$ & $0.92(0.73-1.2)^{c}$ \\
\hline & $k_{c, 3 \rightarrow 2}$ & & & & $0.31(0.25-0.39)^{c}$ & $1.2^{b}$ & $1.4^{c}$ \\
\hline & $k_{c, 4 \rightarrow 3}$ & $19.1(12.2-26.9)$ & $20.8(10.6-32.4)$ & $7.0(4.3-10.9)$ & $7.5^{c}$ & $0.96^{b}$ & $0.69^{c}$ \\
\hline & $\chi^{2} / D O F$ & 2.81 & 1.70 & 3.16 & 2.85 & 3.03 & 2.10 \\
\hline \multirow{10}{*}{$\begin{array}{c}\text { Model } \\
2\end{array}$} & $k_{\text {on }}$ & $25.4(22.9-28.0)^{a}$ & $8.0(7.4-8.6)^{a}$ & $4.7(4.1-5.3)^{a}$ & $4.2(3.6-4.7)^{a}$ & 19.2 * & $18.9(16.5-22.0)^{a}$ \\
\hline & $k_{\text {off }}$ & $20.0^{a}$ & $19.5^{a}$ & $18.6^{a}$ & $9.4^{a}$ & 16.9 * & $71.9^{a}$ \\
\hline & $k_{c, 0 \rightarrow 1}$ & $65.6^{a}$ & $8.9^{a}$ & $6.5^{a}$ & $1.2^{a}$ & $2.6(2.4-2.9)^{a}$ & $2.6^{a}$ \\
\hline & $k_{c, 1 \rightarrow 2}$ & $13.9(12.5-15.5)$ & $7.1(6.3-7.8)$ & $8.0(7.0-9.3)$ & $1.4(1.1-1.7)$ & $0.89(0.81-0.98)^{b}$ & $1.6(1.4-2.0)$ \\
\hline & $k_{c, 2 \rightarrow 3}$ & $37.1(32.9-42.3)^{b}$ & $21.4(19.5-26.8)^{b}$ & $13.1(10.5-16.3)^{b}$ & $2.4(1.9-3.0)^{b}$ & $1.5(1.3-1.7)^{c}$ & $3.4(2.7-4.3)^{b}$ \\
\hline & $k_{c, 3 \rightarrow 4}$ & $373^{b}$ & $214^{b}$ & $132^{b}$ & $23.9^{b}$ & $3.0(2.4-3.8)$ & $3.0^{b}$ \\
\hline & $k_{c, 0 \rightarrow 1 b}$ & & & & & $0.44^{a}$ & $1.0(0.77-1.4)^{c}$ \\
\hline & $k_{c, 1 \rightarrow 2 b}$ & & & & $0.06(0.04-0.09)$ & $0.24^{b}$ & $0.10^{c}$ \\
\hline & $k_{c, 2 \rightarrow 3 b}$ & $1.8(1.1-2.5)$ & $1.9(0.97-3.0)$ & $0.64(0.39-1.0)$ & $0.63(0.52-0.80)$ & $0.73^{c}$ & $0.08^{c}$ \\
\hline & $\chi^{2} / D O F$ & 2.81 & 1.71 & 3.19 & 2.23 & 4.65 & 2.01 \\
\hline \multirow{11}{*}{$\begin{array}{c}\text { Model } \\
3\end{array}$} & $\bar{k}_{\mathrm{on}, 0}$ & $27.6(25.5-30.4)^{a}$ & $8.39(7.8-9.0)^{a}$ & $3.1(2.7-3.6)^{a}$ & $7.9(6.3-10.8)^{a}$ & $33.0(30.8-35.5)^{a}$ & $6.9(6.3-7.5)^{a}$ \\
\hline & $k_{\mathrm{off}, 0}$ & $21.7^{a}$ & $20.6^{a}$ & $12.3^{a}$ & $17.8^{a}$ & $29.1^{a}$ & $26.2^{a}$ \\
\hline & $k_{\mathrm{on}, 1}$ & & & & $5.4^{a}$ & $29.7^{a}$ & $1.5^{a}$ \\
\hline & $k_{\mathrm{on}, 2}$ & & $8.4^{a}$ & & $4.1^{a}$ & $8.3^{a}$ & $0.59^{a}$ \\
\hline & $k_{\mathrm{on}, 3}$ & $0.10^{a}$ & $0.01^{a}$ & $0.05^{a}$ & $0.01^{a}$ & $3.9^{a}$ & $0.14^{a}$ \\
\hline & $k_{\mathrm{on}, 4}$ & $19.9^{a}$ & $5.2^{a}$ & $8.1^{a}$ & $37.2^{a}$ & $271^{a}$ & $11.2^{a}$ \\
\hline & $k_{c, 0 \rightarrow 1}$ & $38.3^{a}$ & $7.5^{a}$ & $13.4^{a}$ & $1.3(1.1-1.6)$ & $2.8^{a}$ & $1.6^{a}$ \\
\hline & $k_{c, 1 \rightarrow 2}$ & $12.5(11.5-13.7)$ & $6.2(5.8-6.7)$ & $10.0(8.7-11.6)$ & $2.8(2.1-3.8)$ & $1.2(1.1-1.3)$ & $4.0(3.6-5.1)$ \\
\hline & $k_{c, 2 \rightarrow 3}$ & $35.4(32.5-38.7)^{b}$ & $20.6(18.0-24.1)^{b}$ & $17.9(15.8-22.5)^{b}$ & $6.2(5.0-7.8)^{b}$ & $6.1(5.7-6.6)$ & $19.7(15.8-24.7)$ \\
\hline & $k_{c, 3 \rightarrow 4}$ & $356^{b}$ & $206^{b}$ & $179^{b}$ & $62.1^{b}$ & $10.5(9.6-11.5)$ & $21.5(19.3-26.3)$ \\
\hline & $\chi^{2} / D O F$ & 2.41 & 1.38 & 2.63 & 3.34 & 3.33 & 1.97 \\
\hline
\end{tabular}

${ }^{*} k_{o n}$ is reported in units of $\mu \mathrm{M}^{-1} \mathrm{~min}^{-1}$. All other apparent rate constants and their corresponding FitSpace boundaries (shown in parentheses) are in units of $\mathrm{min}^{-1}$. ${ }^{\mathrm{a}-\mathrm{c}}$ Indicates rates that were linked at a fixed ratio and were varied as single parameter in the global fit. In model 3 , unless otherwise specified, all $k_{o n, n}=k_{o n, 0}$ and all $k_{o f f, n}=k_{o f f, 0}$. 
Table S4. Simulated kinetic parameters for the HalM2GS389-396 enzyme. Data were simulated with the mechanisms shown in Figures S2 and S3. $k_{o n}$ is in units of $\mu \mathrm{M}^{-1} \mathrm{~min}^{-}$ 1. All rates and their corresponding FitSpace boundaries (shown in parentheses) are in units $\mathrm{min}^{-1}$. Rates that were linked at a constant ratio and were varied as a single parameter during the global fit are indicated with superscripts (a-e).

\begin{tabular}{|c|c|}
\hline \multicolumn{2}{|c|}{ Cyclization } \\
\hline Step & Rate \\
\hline$k_{\text {on }}$ & $4.4(4.0-4.8)^{a}$ \\
\hline$k_{\text {off }}$ & $19.0^{a}$ \\
\hline$k_{c, 0 \rightarrow 1}$ & $0.41^{a}$ \\
\hline$k_{c, 1 \rightarrow 2}$ & $0.38(0.33-0.43)$ \\
\hline$k_{c, 2 \rightarrow 3}$ & $5.3(4.5-6.4)$ \\
\hline$k_{c, 3 \rightarrow 4}$ & $1.1(0.96-1.3)$ \\
\hline$k_{i 0}$ & $0.11(0.09-0.13)^{b}$ \\
\hline$k_{i 1}$ & $0.13^{b}$ \\
\hline$k_{i 2}$ & $2.2^{b}$ \\
\hline$k_{i 3}$ & $1.5^{b}$ \\
\hline$\chi^{2} / D O F$ & 3.65 \\
\hline \multicolumn{2}{|c|}{ Dehydration } \\
\hline Step & Rate \\
\hline$k_{\text {on }}$ & $4.4(3.8-5.3)^{a}$ \\
\hline$k_{\text {off }}$ & $19^{a}$ \\
\hline$k_{d, 0 \rightarrow 1}$ & $46.2^{a}$ \\
\hline$k_{p, 0 \rightarrow 1}$ & $12.1^{a}$ \\
\hline$k_{e, 0 \rightarrow 1}$ & $1.0^{a}$ \\
\hline$k_{d, 1 \rightarrow 2}$ & $6.5(5.7-7.7)^{b}$ \\
\hline$k_{p, 1 \rightarrow 2}$ & $0.83^{b}$ \\
\hline$k_{e, 1 \rightarrow 2}$ & $0.34^{b}$ \\
\hline$k_{d, 2 \rightarrow 3}$ & $1.1(0.96-1.4)^{c}$ \\
\hline$k_{p, 2 \rightarrow 3}$ & $0.05^{c}$ \\
\hline$k_{e, 2 \rightarrow 3}$ & $0.01^{c}$ \\
\hline$k_{d, 3 \rightarrow 4}$ & $1.7(1.5-2.2)^{d}$ \\
\hline$k_{d, 4 \rightarrow 5}$ & $1.1^{d}$ \\
\hline$k_{d, 5 \rightarrow 6}$ & $0.74(0.60-1.0)^{e}$ \\
\hline$k_{d, 6 \rightarrow 7}$ & $0.74^{e}$ \\
\hline$k_{\text {inact }}$ & $0.09(0.07-0.12)$ \\
\hline$\chi^{2} / D O F$ & 7.11 \\
\hline
\end{tabular}


Table S5. List of HalM2-derived peptides that undergo significant changes in deuterium uptake relative to wt HalM2.

\begin{tabular}{|c|c|c|c|c|}
\hline \multirow{2}{*}{ Sequence } & \multicolumn{4}{|c|}{ 2] - [HalM2 variant] } \\
\hline & Start & End & $\begin{array}{l}\text { Relative } \\
\text { Uptake } \\
\text { Difference }\end{array}$ & $\begin{array}{l}\text { Relative } \\
\text { Uptake } \\
\text { SD }\end{array}$ \\
\hline \multicolumn{5}{|c|}{ [WT-HalM2] - [GS331-340] } \\
\hline VEQPYVTESSQNKPTVNGKEAN & 392 & 413 & 1.07 & 0.24 \\
\hline SAVGGGKGVQSE & 363 & 374 & 0.72 & 0.4 \\
\hline SSQNKPTVNGKEANIGNYIPHVTDG & 400 & 424 & -0.41 & 0.39 \\
\hline LFTNDLDC & 317 & 324 & -0.45 & 0.14 \\
\hline VSVGMNE & 629 & 635 & -0.47 & 0.15 \\
\hline AVTVSPLTPGIYDGTL & 636 & 651 & -0.48 & 0.37 \\
\hline YLFGATDL & 290 & 297 & -0.52 & 0.19 \\
\hline LFGATDL & 291 & 297 & -0.56 & 0.19 \\
\hline EAVTVSPLTPGIYDGTL & 635 & 651 & -0.57 & 0.53 \\
\hline FGATDLHL & 292 & 299 & -0.57 & 0.33 \\
\hline VSVGMN & 629 & 634 & -0.6 & 0.13 \\
\hline LGVTGIGYQL & 950 & 959 & -0.69 & 0.14 \\
\hline EAVTVSPLTPGIYDGTLG & 635 & 652 & -0.76 & 0.33 \\
\hline GTLMLPITIASGKL & 344 & 357 & -0.87 & 0.27 \\
\hline IRYIKSSL & 564 & 571 & -0.91 & 0.18 \\
\hline AVTVSPLTPGIYDGTLG & 636 & 652 & -0.93 & 0.33 \\
\hline AVTVSPLTPGIY & 636 & 647 & -1.18 & 0.29 \\
\hline EAVTVSPLTPGIY & 635 & 647 & -1.27 & 0.35 \\
\hline ARQIRYIKSSL & 561 & 571 & -1.37 & 0.21 \\
\hline \multicolumn{5}{|c|}{ [WT-HalM2] - [GS491-497] } \\
\hline APFKKIVPHE & 498 & 507 & 1.23 & 0.13 \\
\hline MAPFKKIVPHE & 497 & 507 & 1.18 & 0.14 \\
\hline PFKKIVPHE & 499 & 507 & 1.12 & 0.12 \\
\hline KQLKPELMPSSA & 682 & 693 & 0.41 & 0.13 \\
\hline VSVGMN & 629 & 634 & -0.41 & 0.13 \\
\hline EASTHPDYLQEPTRRNKL & 469 & 486 & -0.42 & 0.13 \\
\hline FVLTCGGTIVKDGYGRD & 519 & 535 & -0.44 & 0.18 \\
\hline YLFGATDL & 290 & 297 & -0.45 & 0.07 \\
\hline VEQPYVTESSQNKPTVNGKEAN & 392 & 413 & -0.48 & 0.34 \\
\hline LFGATDL & 291 & 297 & -0.49 & 0.16 \\
\hline FGATDLHL & 292 & 299 & -0.5 & 0.34 \\
\hline IRYIKSSL & 564 & 571 & -0.51 & 0.15 \\
\hline
\end{tabular}




\begin{tabular}{|c|c|c|c|c|}
\hline LQEPTRRNKL & 477 & 486 & -0.54 & 0.21 \\
\hline STHPDYLQEPTRRNKL & 471 & 486 & -0.57 & 0.3 \\
\hline AVTVSPLTPGIY & 636 & 647 & -0.6 & 0.35 \\
\hline EAVTVSPLTPGIY & 635 & 647 & -0.61 & 0.27 \\
\hline GTLMLPITIASGKLLD & 344 & 359 & -0.61 & 0.28 \\
\hline ASTHPDYLQEPTRRNKL & 470 & 486 & -0.61 & 0.15 \\
\hline VEQPYVTESSQNKPTVNGKE & 392 & 411 & -0.62 & 0.33 \\
\hline IEWVNSKGFQPSLRI & 235 & 249 & -0.71 & 0.43 \\
\hline LFQSSC & 539 & 544 & -0.72 & 0.1 \\
\hline ARQIRYIKSSL & 561 & 571 & -0.81 & 0.16 \\
\hline GTLMLPITIASGKL & 344 & 357 & -0.85 & 0.26 \\
\hline AVTVSPLTPGIYDGTLG & 636 & 652 & -1.06 & 0.26 \\
\hline AVTVSPLTPGIYDGTL & 636 & 651 & -1.11 & 0.3 \\
\hline EAVTVSPLTPGIYDGTL & 635 & 651 & -1.13 & 0.19 \\
\hline EAVTVSPLTPGIYDGTLG & 635 & 652 & -1.17 & 0.33 \\
\hline ARELTQSVF & 335 & 343 & -1.54 & 0.25 \\
\hline \multicolumn{5}{|c|}{ [WT-HalM2] - [GS360-365] } \\
\hline PITIASGKL & 349 & 357 & 0.99 & 0.15 \\
\hline KQLKPELMPSSA & 682 & 693 & 0.4 & 0.14 \\
\hline YEFIPHQEATSE & 261 & 272 & -0.42 & 0.13 \\
\hline ANIGNYIPHVTDG & 412 & 424 & -0.43 & 0.15 \\
\hline LFTNDL & 317 & 322 & -0.43 & 0.1 \\
\hline IEWVNSKGFQPSLRI & 235 & 249 & -0.5 & 0.39 \\
\hline LFQSSC & 539 & 544 & -0.56 & 0.14 \\
\hline VSVGMNE & 629 & 635 & -0.56 & 0.11 \\
\hline WVIVNQ & 379 & 384 & -0.56 & 0.18 \\
\hline FGATDLHL & 292 & 299 & -0.61 & 0.34 \\
\hline IRYIKSSL & 564 & 571 & -0.62 & 0.12 \\
\hline LFGATDL & 291 & 297 & -0.64 & 0.15 \\
\hline VSVGMN & 629 & 634 & -0.65 & 0.12 \\
\hline YLFGATDL & 290 & 297 & -0.69 & 0.05 \\
\hline ARQIRYIKSSL & 561 & 571 & -0.96 & 0.18 \\
\hline AVTVSPLTPGIYDGTL & 636 & 651 & -1.41 & 0.27 \\
\hline EAVTVSPLTPGIYDGTL & 635 & 651 & -1.52 & 0.24 \\
\hline EAVTVSPLTPGIY & 635 & 647 & -1.63 & 0.31 \\
\hline AVTVSPLTPGIY & 636 & 647 & -1.75 & 0.32 \\
\hline AVTVSPLTPGIYDGTLG & 636 & 652 & -1.88 & 0.31 \\
\hline EAVTVSPLTPGIYDGTLG & 635 & 652 & -1.98 & 0.4 \\
\hline ARELTQSVF & 335 & 343 & -2.2 & 0.19 \\
\hline \multicolumn{5}{|c|}{ [WT-HalM2] - [GS635-644] } \\
\hline VSVGMN & 629 & 634 & 1.13 & 0.14 \\
\hline
\end{tabular}




\begin{tabular}{|c|c|c|c|c|}
\hline IQKYFHAENDLF & 40 & 51 & 0.41 & 0.16 \\
\hline LFTNDL & 317 & 322 & -0.41 & 0.09 \\
\hline FKQLKPELMPSSAY & 681 & 694 & -0.42 & 0.22 \\
\hline ARQIRY & 561 & 566 & -0.42 & 0.15 \\
\hline IDQAHTYGWKLGL & 927 & 939 & -0.42 & 0.22 \\
\hline VSGLSGVLYM & 740 & 749 & -0.43 & 0.15 \\
\hline ALALLT & 797 & 802 & -0.44 & 0.07 \\
\hline ISRLLL & 856 & 861 & -0.45 & 0.27 \\
\hline VYERNRF & 821 & 827 & -0.45 & 0.34 \\
\hline YEFIPHQEATSE & 261 & 272 & -0.45 & 0.11 \\
\hline IERVTHRLQQLGSED & 545 & 559 & -0.47 & 0.37 \\
\hline GVTGIGYQLL & 951 & 960 & -0.49 & 0.17 \\
\hline RVTHRLQQLGSED & 547 & 559 & -0.5 & 0.14 \\
\hline IWEDDRHAAY & 616 & 625 & -0.51 & 0.09 \\
\hline WCHGAPGIG & 847 & 855 & -0.54 & 0.36 \\
\hline GVTGIG & 951 & 956 & -0.56 & 0.06 \\
\hline YLFGATDL & 290 & 297 & -0.56 & 0.08 \\
\hline QAHTYGWKLGLNHSDQLQG & 929 & 947 & -0.58 & 0.28 \\
\hline LFQSSC & 539 & 544 & -0.58 & 0.09 \\
\hline IERVTHRLQQLGSEDE & 545 & 560 & -0.6 & 0.36 \\
\hline YERNRF & 822 & 827 & -0.61 & 0.08 \\
\hline GLSHGAAGF & 788 & 796 & -0.62 & 0.04 \\
\hline HGDFGNLDL & 894 & 902 & -0.63 & 0.12 \\
\hline FGATDLHL & 292 & 299 & -0.64 & 0.31 \\
\hline ANIGNYIPHVTDG & 412 & 424 & -0.65 & 0.2 \\
\hline YAQYTNNPEPKELARKLAISS & 906 & 926 & -0.66 & 0.21 \\
\hline LFGATDL & 291 & 297 & -0.66 & 0.15 \\
\hline LHEELNA & 870 & 876 & -0.7 & 0.09 \\
\hline IWEDDRHAAYL & 616 & 626 & -0.72 & 0.38 \\
\hline IEWVNSKGFQPSLRI & 235 & 249 & -0.75 & 0.33 \\
\hline TGLSHGAAGFA & 787 & 797 & -0.75 & 0.29 \\
\hline LIWEDDRHAAYL & 615 & 626 & -0.85 & 0.16 \\
\hline IRYIKSSL & 564 & 571 & -0.89 & 0.12 \\
\hline ELNAAL & 873 & 878 & -0.91 & 0.07 \\
\hline LARKLAISSID & 918 & 928 & -0.91 & 0.09 \\
\hline GTLMLPITIASGKLLD & 344 & 359 & -0.93 & 0.25 \\
\hline LARKLAISSIDQAHTYG & 918 & 934 & -1.08 & 0.13 \\
\hline LGVTGIGYQL & 950 & 959 & -1.08 & 0.13 \\
\hline GTLMLPITIASGKL & 344 & 357 & -1.13 & 0.26 \\
\hline NKTISDGFGHNHSLCHGDFGNL & 879 & 900 & -1.3 & 0.26 \\
\hline ARQIRYIKSSL & 561 & 571 & -1.47 & 0.16 \\
\hline
\end{tabular}




\begin{tabular}{|c|c|c|c|c|}
\hline ARELTQSVF & 335 & 343 & -2.07 & 0.19 \\
\hline \multicolumn{5}{|c|}{ [WT-HalM2] - [T386A/D387A] } \\
\hline ALELPSSTLTEKELRIHDR & 972 & 990 & 0.88 & 0.31 \\
\hline KQLKPELMPSSA & 682 & 693 & 0.59 & 0.15 \\
\hline ATLTNGDWTPSHEKTPMSPASADREDGYF & 572 & 600 & 0.52 & 0.29 \\
\hline LEASTHPDYLQEPTRRNKL & 468 & 486 & 0.43 & 0.17 \\
\hline FASGQKVVYKPRSLSIDKQFGEF & 212 & 234 & 0.41 & 0.35 \\
\hline YEFIPHQEATSE & 261 & 272 & -0.41 & 0.18 \\
\hline RVTHRLQQLGSED & 547 & 559 & -0.45 & 0.24 \\
\hline IDQAHTYGWKLGL & 927 & 939 & -0.47 & 0.28 \\
\hline FGATDLHL & 292 & 299 & -0.47 & 0.39 \\
\hline QAHTYGWKLGLNHSDQLQG & 929 & 947 & -0.55 & 0.4 \\
\hline MHIDKL & 109 & 114 & -0.55 & 0.1 \\
\hline YLFGATDL & 290 & 297 & -0.57 & 0.1 \\
\hline LFQSSC & 539 & 544 & -0.58 & 0.12 \\
\hline LFGATDL & 291 & 297 & -0.65 & 0.16 \\
\hline IRYIKSSL & 564 & 571 & -0.65 & 0.15 \\
\hline VEQPYVTESSQNKPTVNGKEAN & 392 & 413 & -0.66 & 0.18 \\
\hline VSVGMNE & 629 & 635 & -0.68 & 0.13 \\
\hline VSVGMN & 629 & 634 & -0.75 & 0.14 \\
\hline SSQNKPTVNGKEANIGNYIPHVTDG & 400 & 424 & -0.79 & 0.29 \\
\hline ARQIRYIKSSL & 561 & 571 & -0.99 & 0.2 \\
\hline IEWVNSKGFQPSLRI & 235 & 249 & -1 & 0.41 \\
\hline VEQPYVTESSQNKPTVNGKE & 392 & 411 & -1.04 & 0.36 \\
\hline ANIGNYIPHVTDG & 412 & 424 & -1.08 & 0.23 \\
\hline WVIVNQ & 379 & 384 & -1.18 & 0.17 \\
\hline TVGLNGSTPHERYMDF & 115 & 130 & -1.2 & 0.36 \\
\hline NGSTPHERYMDF & 119 & 130 & -1.22 & 0.17 \\
\hline GTLMLPITIASGKLLD & 344 & 359 & -1.31 & 0.28 \\
\hline GTLMLPITIASGKL & 344 & 357 & -1.4 & 0.29 \\
\hline EAVTVSPLTPGIY & 635 & 647 & -2.01 & 0.29 \\
\hline AVTVSPLTPGIY & 636 & 647 & -2.08 & 0.31 \\
\hline ARELTQSVF & 335 & 343 & -2.22 & 0.2 \\
\hline AVTVSPLTPGIYDGTLG & 636 & 652 & -2.65 & 0.33 \\
\hline AVTVSPLTPGIYDGTL & 636 & 651 & -2.79 & 0.37 \\
\hline EAVTVSPLTPGIYDGTLG & 635 & 652 & -2.89 & 0.38 \\
\hline EAVTVSPLTPGIYDGTL & 635 & 651 & -3.33 & 0.17 \\
\hline \multicolumn{5}{|c|}{ [WT-HalM2] - [GS389-396] } \\
\hline ALELPSSTLTEKELRIHDR & 972 & 990 & 0.68 & 0.32 \\
\hline KQLKPELMPSSA & 682 & 693 & 0.65 & 0.1 \\
\hline ATLTNGDWTPSHEKTPMSPASADREDGYF & 572 & 600 & 0.54 & 0.25 \\
\hline
\end{tabular}




\begin{tabular}{|c|c|c|c|c|}
\hline LEASTHPDYLQEPTRRNKL & 468 & 486 & 0.47 & 0.1 \\
\hline LFQSSC & 539 & 544 & -0.42 & 0.15 \\
\hline MHIDKL & 109 & 114 & -0.53 & 0.06 \\
\hline VSVGMNE & 629 & 635 & -0.56 & 0.13 \\
\hline IRYIKSSL & 564 & 571 & -0.56 & 0.12 \\
\hline YLFGATDL & 290 & 297 & -0.58 & 0.08 \\
\hline VSVGMN & 629 & 634 & -0.58 & 0.13 \\
\hline LFGATDL & 291 & 297 & -0.6 & 0.16 \\
\hline IEWVNSKGFQPSLRI & 235 & 249 & -0.63 & 0.39 \\
\hline GTLMLPITIASGKLLD & 344 & 359 & -0.79 & 0.29 \\
\hline ANIGNYIPHVTDG & 412 & 424 & -0.85 & 0.17 \\
\hline ARQIRYIKSSL & 561 & 571 & -0.88 & 0.15 \\
\hline TVGLNGSTPHERYMDF & 115 & 130 & -0.99 & 0.34 \\
\hline NGSTPHERYMDF & 119 & 130 & -1.14 & 0.11 \\
\hline GTLMLPITIASGKL & 344 & 357 & -1.24 & 0.27 \\
\hline EAVTVSPLTPGIYDGTL & 635 & 651 & -1.25 & 0.45 \\
\hline AVTVSPLTPGIYDGTL & 636 & 651 & -1.47 & 0.42 \\
\hline AVTVSPLTPGIYDGTLG & 636 & 652 & -1.59 & 0.34 \\
\hline EAVTVSPLTPGIY & 635 & 647 & -1.6 & 0.3 \\
\hline AVTVSPLTPGIY & 636 & 647 & -1.72 & 0.3 \\
\hline EAVTVSPLTPGIYDGTLG & 635 & 652 & -1.73 & 0.45 \\
\hline ARELTQSVF & 335 & 343 & -2.08 & 0.19 \\
\hline \multicolumn{5}{|c|}{ [WT-HalM2] - [GS341-348] } \\
\hline PITIASGKL & 349 & 357 & 2.42 & 0.12 \\
\hline ATLTNGDWTPSHEKTPMSPASADREDGYF & 572 & 600 & 0.83 & 0.22 \\
\hline KQLKPELMPSSA & 682 & 693 & 0.64 & 0.11 \\
\hline ALELPSSTLTEKELRIHDR & 972 & 990 & 0.64 & 0.26 \\
\hline IQKYFHAENDLF & 40 & 51 & 0.59 & 0.13 \\
\hline LEASTHPDYLQEPTRRNKL & 468 & 486 & 0.53 & 0.1 \\
\hline EASTHPDYLQEPTRRNKL & 469 & 486 & 0.47 & 0.13 \\
\hline MKTPLTSEHPSVPTTLPHTNDTDWL & 1 & 25 & 0.43 & 0.28 \\
\hline WVIVNQ & 379 & 384 & -0.42 & 0.17 \\
\hline FGATDLHL & 292 & 299 & -0.44 & 0.39 \\
\hline VEQPYVTESSQNKPTVNGKEAN & 392 & 413 & -0.45 & 0.32 \\
\hline LGVTGIGYQL & 950 & 959 & -0.45 & 0.12 \\
\hline QAHTYGWKLGLNHSDQLQG & 929 & 947 & -0.47 & 0.32 \\
\hline LARKLAISSIDQAHTYG & 918 & 934 & -0.49 & 0.13 \\
\hline IDQAHTYGWKLGL & 927 & 939 & -0.49 & 0.23 \\
\hline RVTHRLQQLGSED & 547 & 559 & -0.53 & 0.15 \\
\hline LFQSSC & 539 & 544 & -0.55 & 0.12 \\
\hline ARQIRY & 561 & 566 & -0.56 & 0.19 \\
\hline
\end{tabular}




\begin{tabular}{|c|c|c|c|c|}
\hline VSVGMNE & 629 & 635 & -0.59 & 0.1 \\
\hline VEQPYVTESSQNKPTVNGKE & 392 & 411 & -0.6 & 0.28 \\
\hline VSVGMN & 629 & 634 & -0.6 & 0.12 \\
\hline YLFGATDL & 290 & 297 & -0.68 & 0.07 \\
\hline LFGATDL & 291 & 297 & -0.7 & 0.15 \\
\hline ANIGNYIPHVTDG & 412 & 424 & -0.77 & 0.15 \\
\hline IEWVNSKGFQPSLRI & 235 & 249 & -0.94 & 0.33 \\
\hline IRYIKSSL & 564 & 571 & -0.99 & 0.11 \\
\hline ARQIRYIKSSL & 561 & 571 & -1.71 & 0.15 \\
\hline EAVTVSPLTPGIY & 635 & 647 & -1.94 & 0.28 \\
\hline AVTVSPLTPGIY & 636 & 647 & -2.12 & 0.27 \\
\hline AVTVSPLTPGIYDGTLG & 636 & 652 & -2.48 & 0.25 \\
\hline AVTVSPLTPGIYDGTL & 636 & 651 & -2.59 & 0.29 \\
\hline EAVTVSPLTPGIYDGTLG & 635 & 652 & -2.83 & 0.32 \\
\hline EAVTVSPLTPGIYDGTL & 635 & 651 & -2.88 & 0.15 \\
\hline \multicolumn{5}{|c|}{ [WT-HalM2] - [P349A] } \\
\hline ATLTNGDWTPSHEKTPMSPASADREDGYF & 572 & 600 & 1.44 & 0.22 \\
\hline VEQPYVTESSQNKPTVNGKEAN & 392 & 413 & 1.26 & 0.19 \\
\hline ALELPSSTLTEKELRIHDR & 972 & 990 & 1.08 & 0.26 \\
\hline MKTPLTSEHPSVPTTLPHTNDTDWL & 1 & 25 & 1.04 & 0.29 \\
\hline ATLTNGDWTPSHEKTPMSPASA & 572 & 593 & 1 & 0.19 \\
\hline SAVGGGKGVQSE & 363 & 374 & 0.95 & 0.37 \\
\hline KQLKPELMPSSA & 682 & 693 & 0.81 & 0.1 \\
\hline MKTPLTSEHPSVPTTLPHTNDTDW & 1 & 24 & 0.79 & 0.33 \\
\hline VEQPYVTESSQNKPTVNGKE & 392 & 411 & 0.7 & 0.28 \\
\hline LEASTHPDYLQEPTRRNKL & 468 & 486 & 0.67 & 0.11 \\
\hline IQKYFHAENDLF & 40 & 51 & 0.66 & 0.14 \\
\hline ALELPSSTLTEKE & 972 & 984 & 0.66 & 0.18 \\
\hline WVDLRKGNAYQTF & 834 & 846 & 0.63 & 0.18 \\
\hline EGMFKQLKPELMPSSA & 678 & 693 & 0.63 & 0.09 \\
\hline EASTHPDYLQEPTRRNKL & 469 & 486 & 0.62 & 0.13 \\
\hline EIQKYFHAENDL & 39 & 50 & 0.59 & 0.13 \\
\hline FFKEKDLRLTNL & 182 & 193 & 0.56 & 0.22 \\
\hline SSQNKPTVNGKEANIGNYIPHVTDG & 400 & 424 & 0.55 & 0.3 \\
\hline VDLRKGNAYQTF & 835 & 846 & 0.52 & 0.17 \\
\hline ASTHPDYLQEPTRRNKL & 470 & 486 & 0.52 & 0.13 \\
\hline FFKEKDLRL & 182 & 190 & 0.52 & 0.24 \\
\hline LTEKELRIHDR & 980 & 990 & 0.51 & 0.08 \\
\hline EFIPHQEA & 262 & 269 & 0.51 & 0.22 \\
\hline PTRRNKL & 480 & 486 & 0.48 & 0.14 \\
\hline FASGQKVVYKPRSLSIDKQFGEF & 212 & 234 & 0.48 & 0.27 \\
\hline
\end{tabular}




\begin{tabular}{|c|c|c|c|c|}
\hline STHPDYLQEPTRRNKL & 471 & 486 & 0.47 & 0.3 \\
\hline RKGNAYQTF & 838 & 846 & 0.41 & 0.3 \\
\hline LYAQYTNNPEPKE & 905 & 917 & 0.41 & 0.27 \\
\hline FASGQKVVYKPRSLSI & 212 & 227 & 0.41 & 0.17 \\
\hline LELPSST & 973 & 979 & 0.41 & 0.09 \\
\hline YLFGATDL & 290 & 297 & -0.48 & 0.09 \\
\hline VSVGMN & 629 & 634 & -0.48 & 0.13 \\
\hline LFGATDL & 291 & 297 & -0.5 & 0.16 \\
\hline IRYIKSSL & 564 & 571 & -0.61 & 0.16 \\
\hline EAVTVSPLTPGIYDGTL & 635 & 651 & -0.85 & 0.15 \\
\hline ARQIRYIKSSL & 561 & 571 & -0.98 & 0.14 \\
\hline AVTVSPLTPGIYDGTL & 636 & 651 & -1.02 & 0.29 \\
\hline AVTVSPLTPGIYDGTLG & 636 & 652 & -1.05 & 0.25 \\
\hline EAVTVSPLTPGIYDGTLG & 635 & 652 & -1.08 & 0.31 \\
\hline EAVTVSPLTPGIY & 635 & 647 & -1.08 & 0.26 \\
\hline AVTVSPLTPGIY & 636 & 647 & -1.1 & 0.28 \\
\hline ARELTQSVF & 335 & 343 & -1.92 & 0.19 \\
\hline \multicolumn{5}{|c|}{ [WT-HalM2] - [P405A] } \\
\hline ATLTNGDWTPSHEKTPMSPASADREDGYF & 572 & 600 & 0.86 & 0.23 \\
\hline ALELPSSTLTEKELRIHDR & 972 & 990 & 0.78 & 0.26 \\
\hline KQLKPELMPSSA & 682 & 693 & 0.69 & 0.1 \\
\hline IQKYFHAENDLF & 40 & 51 & 0.58 & 0.13 \\
\hline EGMFKQLKPELMPSSA & 678 & 693 & 0.5 & 0.11 \\
\hline FASGQKVVYKPRSLSIDKQFGEF & 212 & 234 & 0.45 & 0.27 \\
\hline GTLMLPITIASGKLLD & 344 & 359 & -0.46 & 0.25 \\
\hline LFQSSC & 539 & 544 & -0.47 & 0.1 \\
\hline SAVGGGKGVQSE & 363 & 374 & -0.48 & 0.4 \\
\hline AVTVSPLTPGIY & 636 & 647 & -0.49 & 0.27 \\
\hline EAVTVSPLTPGIY & 635 & 647 & -0.51 & 0.29 \\
\hline GTLMLPITIASGKL & 344 & 357 & -0.56 & 0.27 \\
\hline ANIGNYIPHVTDG & 412 & 424 & -0.58 & 0.14 \\
\hline ARELTQSVF & 335 & 343 & -0.64 & 0.19 \\
\hline AVTVSPLTPGIYDGTLG & 636 & 652 & -0.78 & 0.24 \\
\hline IEWVNSKGFQPSLRI & 235 & 249 & -0.8 & 0.34 \\
\hline EAVTVSPLTPGIYDGTLG & 635 & 652 & -1 & 0.3 \\
\hline AVTVSPLTPGIYDGTL & 636 & 651 & -1.07 & 0.26 \\
\hline EAVTVSPLTPGIYDGTL & 635 & 651 & -1.35 & 0.1 \\
\hline
\end{tabular}


Table S6. List of HalM2-derived peptides that undergo significant changes in deuterium uptake upon binding of AMP-PNP and HalA2.

\section{[Free Enzyme] - [Enzyme: AMPPNP: HalA2]}

\begin{tabular}{|c|c|c|c|c|}
\hline Sequence & Start End & & $\begin{array}{l}\text { Relative } \\
\text { Uptake } \\
\text { Difference }\end{array}$ & $\begin{array}{l}\text { Relative } \\
\text { Uptake SD }\end{array}$ \\
\hline [HalM2-WT] - [HalM & WT: AMPPI & HalA & & \\
\hline ARELTQSVF & 335 & 343 & 1.82 & 0.19 \\
\hline GTLMLPITIASGKLLD & 344 & 359 & 1.34 & 0.26 \\
\hline VEQPYVTESSQNKPTVNGKE & 392 & 411 & 1.27 & 0.35 \\
\hline VEQPYVTESSQNKPTVNGKEAN & 392 & 413 & 1.23 & 0.18 \\
\hline GTLMLPITIASGKL & 344 & 357 & 1.16 & 0.28 \\
\hline ALELPSSTLTEKELRIHDR & 972 & 990 & 1.14 & 0.27 \\
\hline HIDKLTVGL & 110 & 118 & 1.13 & 0.3 \\
\hline $\begin{array}{l}\text { ATLTNGDWTPSHEKTPMSPASADREDGY } \\
\mathrm{F}\end{array}$ & 572 & 600 & 1.12 & 0.21 \\
\hline KQLKPELMPSSA & 682 & 693 & 1.07 & 0.13 \\
\hline ASTHPDYLQEPTRRNKL & 470 & 486 & 1.05 & 0.2 \\
\hline AVTVSPLTPGIY & 636 & 647 & 0.94 & 0.29 \\
\hline GGTIVKDGYGRD & 524 & 535 & 0.9 & 0.16 \\
\hline IRYIKSSL & 564 & 571 & 0.87 & 0.12 \\
\hline EAVTVSPLTPGIY & 635 & 647 & 0.87 & 0.26 \\
\hline EASTHPDYLQEPTRRNKL & 469 & 486 & 0.85 & 0.16 \\
\hline EFIPHQEATSED & 262 & 273 & 0.84 & 0.14 \\
\hline EGMFKQLKPELMPSSA & 678 & 693 & 0.82 & 0.09 \\
\hline YEFIPHQEA & 261 & 269 & 0.81 & 0.23 \\
\hline EFIPHQEA & 262 & 269 & 0.79 & 0.09 \\
\hline ALELPSSTLTEKE & 972 & 984 & 0.79 & 0.23 \\
\hline LEASTHPDYLQEPTRRNKL & 468 & 486 & 0.77 & 0.13 \\
\hline MLPITIASGKL & 347 & 357 & 0.76 & 0.13 \\
\hline IWEDDRHAAYL & 616 & 626 & 0.73 & 0.4 \\
\hline YEFIPHQEATSE & 261 & 272 & 0.72 & 0.13 \\
\hline QAHTYGWKLGLNHSDQLQG & 929 & 947 & 0.69 & 0.27 \\
\hline FASGQKVVYKPRSLS & 212 & 226 & 0.68 & 0.22 \\
\hline YLFGATDL & 290 & 297 & 0.66 & 0.07 \\
\hline FGATDLHL & 292 & 299 & 0.64 & 0.35 \\
\hline TVGLNGSTPHERYMDF & 115 & 130 & 0.64 & 0.29 \\
\hline KQLKPELMPSSAY & 682 & 694 & 0.63 & 0.14 \\
\hline STHPDYLQEPTRRNKL & 471 & 486 & 0.62 & 0.32 \\
\hline LPITIASGKL & 348 & 357 & 0.59 & 0.15 \\
\hline
\end{tabular}




\begin{tabular}{|c|c|c|c|c|}
\hline FKQLKPELMPSSAY & 681 & 694 & 0.59 & 0.24 \\
\hline MAPFKKIVPHE & 497 & 507 & 0.57 & 0.17 \\
\hline AVTVSPLTPGIYDGTLG & 636 & 652 & 0.57 & 0.25 \\
\hline LFGATDL & 291 & 297 & 0.56 & 0.15 \\
\hline SSQNKPTVNGKEANIGNYIPHVTDG & 400 & 424 & 0.55 & 0.32 \\
\hline EIQKYFHAENDL & 39 & 50 & 0.54 & 0.19 \\
\hline IQKYFHAENDLF & 40 & 51 & 0.53 & 0.24 \\
\hline ARQIRYIKSSL & 561 & 571 & 0.52 & 0.14 \\
\hline ATLTNGDWTPSHEKTPMSPASA & 572 & 593 & 0.52 & 0.22 \\
\hline FASGQKVVYKPRSLSIDKQFGEF & 212 & 234 & 0.52 & 0.23 \\
\hline LIWEDDRHAAYL & 615 & 626 & 0.51 & 0.18 \\
\hline APFKKIVPHE & 498 & 507 & 0.5 & 0.15 \\
\hline PFKKIVPHE & 499 & 507 & 0.49 & 0.13 \\
\hline LKQGHSYL & 813 & 820 & 0.49 & 0.2 \\
\hline FFKEKDLRLTNL & 182 & 193 & 0.48 & 0.21 \\
\hline VSVGMN & 629 & 634 & 0.47 & 0.13 \\
\hline FASGQKVVYKPRSLSIDKQ & 212 & 230 & 0.47 & 0.24 \\
\hline YAQYTNNPEPKELARKLAISS & 906 & 926 & 0.44 & 0.15 \\
\hline GVGDTHVNGQC & 196 & 206 & 0.44 & 0.22 \\
\hline VSVGMNE & 629 & 635 & 0.43 & 0.1 \\
\hline LIERQSLL & 79 & 86 & 0.43 & 0.15 \\
\hline EAVTVSPLTPGIYDGTLG & 635 & 652 & 0.42 & 0.3 \\
\hline MDHNGPIFA & 440 & 448 & 0.41 & 0.08 \\
\hline FKQLKPELMPSSA & 681 & 693 & 0.41 & 0.31 \\
\hline RVTHRLQQLGSED & 547 & 559 & -0.48 & 0.21 \\
\hline SAVGGGKGVQSE & 363 & 374 & -0.74 & 0.42 \\
\hline \multicolumn{5}{|c|}{ [GS331-340] - [GS331-340: AMPPNP: HalA2] } \\
\hline $\begin{array}{l}\text { ATLTNGDWTPSHEKTPMSPASADREDGY } \\
\mathrm{F}\end{array}$ & 572 & 600 & 1.46 & 0.22 \\
\hline EGMFKQLKPELMPSSA & 678 & 693 & 1.16 & 0.25 \\
\hline ALELPSSTLTEKELRIHDR & 972 & 990 & 1.01 & 0.33 \\
\hline HIDKLTVGL & 110 & 118 & 1 & 0.27 \\
\hline GGTIVKDGYGRD & 524 & 535 & 0.95 & 0.13 \\
\hline EFIPHQEATSED & 262 & 273 & 0.92 & 0.32 \\
\hline FASGQKVVYKPRSLS & 212 & 226 & 0.78 & 0.24 \\
\hline ATLTNGDWTPSHEKTPMSPASA & 572 & 593 & 0.73 & 0.31 \\
\hline TVGLNGSTPHERYMDF & 115 & 130 & 0.72 & 0.37 \\
\hline LEASTHPDYLQEPTRRNKL & 468 & 486 & 0.7 & 0.19 \\
\hline ASTHPDYLQEPTRRNKL & 470 & 486 & 0.68 & 0.13 \\
\hline FASGQKVVYKPRSLSIDKQFGEF & 212 & 234 & 0.66 & 0.27 \\
\hline FKQLKPELMPSSAY & 681 & 694 & 0.62 & 0.28 \\
\hline
\end{tabular}




\begin{tabular}{|c|c|c|c|c|}
\hline EASTHPDYLQEPTRRNKL & 469 & 486 & 0.62 & 0.16 \\
\hline IWEDDRHAAYL & 616 & 626 & 0.6 & 0.4 \\
\hline CGGTIVKDGYGRD & 523 & 535 & 0.57 & 0.19 \\
\hline IQKYFHAENDLF & 40 & 51 & 0.56 & 0.25 \\
\hline FFKEKDLRLTNL & 182 & 193 & 0.56 & 0.36 \\
\hline LKHLEE & 724 & 729 & 0.54 & 0.16 \\
\hline KQLKPELMPSSA & 682 & 693 & 0.54 & 0.2 \\
\hline MHIDKL & 109 & 114 & 0.52 & 0.1 \\
\hline STHPDYLQEPTRRNKL & 471 & 486 & 0.48 & 0.31 \\
\hline FASGQKVVYKPRSLSIDKQ & 212 & 230 & 0.47 & 0.25 \\
\hline FKQLKPELMPSSA & 681 & 693 & 0.47 & 0.24 \\
\hline AQQTGETHYRHAADAL & 661 & 676 & 0.46 & 0.2 \\
\hline AQQTGETHYRHAADALL & 661 & 677 & 0.45 & 0.17 \\
\hline LFTNDL & 317 & 322 & 0.44 & 0.13 \\
\hline MLPITIASGKL & 347 & 357 & 0.44 & 0.31 \\
\hline MKTPLTSEHPSVPTTLPHTNDTDW & 1 & 24 & 0.43 & 0.31 \\
\hline LFTNDLDC & 317 & 324 & 0.42 & 0.08 \\
\hline EIQKYFHAENDL & 39 & 50 & 0.42 & 0.16 \\
\hline MVLGLQRSDSHIIQKAYE & 705 & 722 & 0.41 & 0.28 \\
\hline GTLMLPITIASGKL & 344 & 357 & -0.54 & 0.11 \\
\hline NHSDQLQG & 940 & 947 & -0.55 & 0.11 \\
\hline VEQPYVTESSQNKPTVNGKEAN & 392 & 413 & -0.55 & 0.21 \\
\hline ARQIRY & 561 & 566 & -0.66 & 0.12 \\
\hline IDQAHTYGWKLGL & 927 & 939 & -0.74 & 0.25 \\
\hline AVTVSPLTPGIY & 636 & 647 & -0.74 & 0.14 \\
\hline ANIGNYIPHVTDG & 412 & 424 & -0.75 & 0.23 \\
\hline ARQIRYIKSSL & 561 & 571 & -0.9 & 0.33 \\
\hline IEWVNSKGFQPSLRI & 235 & 249 & -0.92 & 0.18 \\
\hline EAVTVSPLTPGIY & 635 & 647 & -0.94 & 0.28 \\
\hline SAVGGGKGVQSE & 363 & 374 & -1.38 & 0.28 \\
\hline GTLMLPITIASGKLLD & 344 & 359 & -1.71 & 0.1 \\
\hline AVTVSPLTPGIYDGTLG & 636 & 652 & -1.71 & 0.25 \\
\hline AVTVSPLTPGIYDGTL & 636 & 651 & -2.34 & 0.29 \\
\hline EAVTVSPLTPGIYDGTLG & 635 & 652 & -2.38 & 0.17 \\
\hline EAVTVSPLTPGIYDGTL & 635 & 651 & -2.64 & 0.55 \\
\hline \multicolumn{5}{|c|}{ [GS491-497] - [491-497: AMPPNP: HalA2] } \\
\hline ARELTQSVF & 335 & 343 & 2.28 & 0.17 \\
\hline GTLMLPITIASGKL & 344 & 357 & 1.67 & 0.09 \\
\hline GTLMLPITIASGKLLD & 344 & 359 & 1.31 & 0.18 \\
\hline $\begin{array}{l}\text { ATLTNGDWTPSHEKTPMSPASADREDGY } \\
\mathrm{F}\end{array}$ & 572 & 600 & 1.28 & 0.11 \\
\hline
\end{tabular}




\begin{tabular}{|c|c|c|c|c|}
\hline AVTVSPLTPGIY & 636 & 647 & 1.2 & 0.24 \\
\hline VEQPYVTESSQNKPTVNGKEAN & 392 & 413 & 1.08 & 0.32 \\
\hline AVTVSPLTPGIYDGTLG & 636 & 652 & 1.06 & 0.12 \\
\hline ARQIRYIKSSL & 561 & 571 & 1.06 & 0.1 \\
\hline EAVTVSPLTPGIY & 635 & 647 & 1 & 0.15 \\
\hline VEQPYVTESSQNKPTVNGKE & 392 & 411 & 0.99 & 0.2 \\
\hline HIDKLTVGL & 110 & 118 & 0.97 & 0.2 \\
\hline EAVTVSPLTPGIYDGTLG & 635 & 652 & 0.97 & 0.15 \\
\hline IRYIKSSL & 564 & 571 & 0.96 & 0.13 \\
\hline ASTHPDYLQEPTRRNKL & 470 & 486 & 0.92 & 0.13 \\
\hline EGMFKQLKPELMPSSA & 678 & 693 & 0.92 & 0.09 \\
\hline EASTHPDYLQEPTRRNKL & 469 & 486 & 0.91 & 0.09 \\
\hline QAHTYGWKLGLNHSDQLQG & 929 & 947 & 0.9 & 0.24 \\
\hline GGTIVKDGYGRD & 524 & 535 & 0.86 & 0.27 \\
\hline ALELPSSTLTEKELRIHDR & 972 & 990 & 0.86 & 0.23 \\
\hline AVTVSPLTPGIYDGTL & 636 & 651 & 0.85 & 0.17 \\
\hline KQLKPELMPSSAY & 682 & 694 & 0.82 & 0.13 \\
\hline ATLTNGDWTPSHEKTPMSPASA & 572 & 593 & 0.81 & 0.17 \\
\hline EFIPHQEA & 262 & 269 & 0.76 & 0.09 \\
\hline YEFIPHQEATSE & 261 & 272 & 0.73 & 0.03 \\
\hline YLFGATDL & 290 & 297 & 0.72 & 0.06 \\
\hline STHPDYLQEPTRRNKL & 471 & 486 & 0.72 & 0.22 \\
\hline TVGLNGSTPHERYMDF & 115 & 130 & 0.7 & 0.4 \\
\hline FGATDLHL & 292 & 299 & 0.67 & 0.32 \\
\hline LFGATDL & 291 & 297 & 0.67 & 0.09 \\
\hline YEFIPHQEA & 261 & 269 & 0.66 & 0.16 \\
\hline IWEDDRHAAYL & 616 & 626 & 0.64 & 0.4 \\
\hline MLPITIASGKL & 347 & 357 & 0.62 & 0.24 \\
\hline EAVTVSPLTPGIYDGTL & 635 & 651 & 0.6 & 0.19 \\
\hline LEASTHPDYLQEPTRRNKL & 468 & 486 & 0.6 & 0.06 \\
\hline FASGQKVVYKPRSLSIDKQFGEF & 212 & 234 & 0.6 & 0.24 \\
\hline ALELPSSTLTEKE & 972 & 984 & 0.59 & 0.09 \\
\hline SSQNKPTVNGKEANIGNYIPHVTDG & 400 & 424 & 0.58 & 0.2 \\
\hline FKQLKPELMPSSAY & 681 & 694 & 0.58 & 0.18 \\
\hline VSVGMNE & 629 & 635 & 0.56 & 0.07 \\
\hline LYAQYTNNPEPKE & 905 & 917 & 0.56 & 0.35 \\
\hline KQLKPELMPSSA & 682 & 693 & 0.55 & 0.15 \\
\hline MKTPLTSEHPSVPTTLPHTNDTDW & 1 & 24 & 0.54 & 0.23 \\
\hline LFTNDL & 317 & 322 & 0.54 & 0.09 \\
\hline EIQKYFHAENDL & 39 & 50 & 0.53 & 0.07 \\
\hline VSVGMN & 629 & 634 & 0.53 & 0.08 \\
\hline
\end{tabular}




\begin{tabular}{|c|c|c|c|c|}
\hline FKQLKPELMPSSA & 681 & 693 & 0.53 & 0.28 \\
\hline FASGQKVVYKPRSLS & 212 & 226 & 0.51 & 0.08 \\
\hline FASGQKVVYKPRSLSIDKQ & 212 & 230 & 0.5 & 0.21 \\
\hline MHIDKL & 109 & 114 & 0.48 & 0.05 \\
\hline MKTPLTSEHPSVPTTLPHTNDTDWL & 1 & 25 & 0.47 & 0.17 \\
\hline LPITIASGKL & 348 & 357 & 0.47 & 0.12 \\
\hline LFTNDLDC & 317 & 324 & 0.46 & 0.04 \\
\hline LKQGHSYL & 813 & 820 & 0.44 & 0.12 \\
\hline GVGDTHVNGQC & 196 & 206 & 0.43 & 0.13 \\
\hline IQKYFHAENDLF & 40 & 51 & 0.42 & 0.15 \\
\hline FFKEKDLRLTNL & 182 & 193 & 0.41 & 0.12 \\
\hline \multicolumn{5}{|c|}{ [GS360-365] - [GS360-365 : AMPPNP: HalA2] } \\
\hline ARELTQSVF & 335 & 343 & 2.8 & 0.06 \\
\hline AVTVSPLTPGIY & 636 & 647 & 2.05 & 0.18 \\
\hline EAVTVSPLTPGIY & 635 & 647 & 1.73 & 0.21 \\
\hline AVTVSPLTPGIYDGTLG & 636 & 652 & 1.58 & 0.22 \\
\hline EAVTVSPLTPGIYDGTLG & 635 & 652 & 1.49 & 0.28 \\
\hline ARQIRYIKSSL & 561 & 571 & 1.32 & 0.15 \\
\hline HIDKLTVGL & 110 & 118 & 1.24 & 0.25 \\
\hline $\begin{array}{l}\text { ATLTNGDWTPSHEKTPMSPASADREDGY } \\
\mathrm{F}\end{array}$ & 572 & 600 & 1.2 & 0.13 \\
\hline EFIPHQEATSED & 262 & 273 & 1.15 & 0.09 \\
\hline IRYIKSSL & 564 & 571 & 1.04 & 0.07 \\
\hline ALELPSSTLTEKELRIHDR & 972 & 990 & 1.03 & 0.3 \\
\hline EGMFKQLKPELMPSSA & 678 & 693 & 0.99 & 0.12 \\
\hline FASGQKVVYKPRSLS & 212 & 226 & 0.92 & 0.2 \\
\hline LFTNDL & 317 & 322 & 0.9 & 0.08 \\
\hline AVTVSPLTPGIYDGTL & 636 & 651 & 0.9 & 0.13 \\
\hline EAVTVSPLTPGIYDGTL & 635 & 651 & 0.89 & 0.23 \\
\hline IWEDDRHAAYL & 616 & 626 & 0.88 & 0.41 \\
\hline ASTHPDYLQEPTRRNKL & 470 & 486 & 0.87 & 0.1 \\
\hline EASTHPDYLQEPTRRNKL & 469 & 486 & 0.86 & 0.14 \\
\hline TVGLNGSTPHERYMDF & 115 & 130 & 0.83 & 0.37 \\
\hline ALELPSSTLTEKE & 972 & 984 & 0.83 & 0.16 \\
\hline KQLKPELMPSSAY & 682 & 694 & 0.83 & 0.19 \\
\hline NKTISDGFGHNHSLCHGDFGNL & 879 & 900 & 0.82 & 0.06 \\
\hline YLFGATDL & 290 & 297 & 0.77 & 0.04 \\
\hline LFGATDL & 291 & 297 & 0.75 & 0.09 \\
\hline QAHTYGWKLGLNHSDQLQG & 929 & 947 & 0.73 & 0.25 \\
\hline FASGQKVVYKPRSLSIDKQFGEF & 212 & 234 & 0.73 & 0.23 \\
\hline FKQLKPELMPSSAY & 681 & 694 & 0.71 & 0.23 \\
\hline
\end{tabular}




\begin{tabular}{|c|c|c|c|c|}
\hline MAPFKKIVPHE & 497 & 507 & 0.71 & 0.15 \\
\hline KQLKPELMPSSA & 682 & 693 & 0.69 & 0.13 \\
\hline LEASTHPDYLQEPTRRNKL & 468 & 486 & 0.68 & 0.16 \\
\hline IWEDDRHAAY & 616 & 625 & 0.67 & 0.21 \\
\hline MHIDKL & 109 & 114 & 0.67 & 0.09 \\
\hline STHPDYLQEPTRRNKL & 471 & 486 & 0.64 & 0.25 \\
\hline APFKKIVPHE & 498 & 507 & 0.64 & 0.11 \\
\hline LIWEDDRHAAYL & 615 & 626 & 0.61 & 0.21 \\
\hline LFTNDLDC & 317 & 324 & 0.61 & 0.07 \\
\hline FKQLKPELMPSSA & 681 & 693 & 0.61 & 0.21 \\
\hline FFKEKDLRLTNL & 182 & 193 & 0.59 & 0.32 \\
\hline VSVGMN & 629 & 634 & 0.57 & 0.06 \\
\hline PFKKIVPHE & 499 & 507 & 0.57 & 0.07 \\
\hline WVIVNQ & 379 & 384 & 0.56 & 0.09 \\
\hline ATLTNGDWTPSHEKTPMSPASA & 572 & 593 & 0.55 & 0.19 \\
\hline FGATDLHL & 292 & 299 & 0.55 & 0.33 \\
\hline EFIPHQEA & 262 & 269 & 0.55 & 0.11 \\
\hline FASGQKVVYKPRSLSIDKQ & 212 & 230 & 0.54 & 0.22 \\
\hline SSQNKPTVNGKEANIGNYIPHVTDG & 400 & 424 & 0.53 & 0.33 \\
\hline VSVGMNE & 629 & 635 & 0.53 & 0.09 \\
\hline IERVTHRLQQLGSEDE & 545 & 560 & 0.52 & 0.27 \\
\hline YAQYTNNPEPKELARKLAISS & 906 & 926 & 0.51 & 0.15 \\
\hline NGSTPHERYMDF & 119 & 130 & 0.48 & 0.03 \\
\hline EIQKYFHAENDL & 39 & 50 & 0.47 & 0.14 \\
\hline VEQPYVTESSQNKPTVNGKEAN & 392 & 413 & 0.47 & 0.19 \\
\hline GGTIVKDGYGRD & 524 & 535 & 0.44 & 0.11 \\
\hline CGGTIVKDGYGRD & 523 & 535 & 0.43 & 0.14 \\
\hline YEFIPHQEATSE & 261 & 272 & 0.43 & 0.13 \\
\hline FDQLAQQTGETHYRHAADAL & 657 & 676 & 0.43 & 0.14 \\
\hline IQKYFHAENDLF & 40 & 51 & 0.43 & 0.17 \\
\hline FASGQKVVYKPRSLSI & 212 & 227 & 0.43 & 0.21 \\
\hline VEQPYVTESSQNKPTVNGKE & 392 & 411 & 0.41 & 0.09 \\
\hline LREAQAIGDD & 601 & 610 & 0.41 & 0.3 \\
\hline MKTPLTSEHPSVPTTLPHTNDTDW & 1 & 24 & 0.4 & 0.24 \\
\hline MKTPLTSEHPSVPTTLPHTNDTDWL & 1 & 25 & 0.4 & 0.16 \\
\hline PITIASGKL & 349 & 357 & -0.72 & 0.12 \\
\hline \multicolumn{5}{|c|}{ [GS635-644] - [GS635-644: AMPPNP: HaIA2] } \\
\hline HIDKLTVGL & 110 & 118 & 1.19 & 0.26 \\
\hline VEQPYVTESSQNKPTVNGKEAN & 392 & 413 & 0.89 & 0.2 \\
\hline $\begin{array}{l}\text { ATLTNGDWTPSHEKTPMSPASADREDGY } \\
\mathrm{F}\end{array}$ & 572 & 600 & 0.84 & 0.28 \\
\hline
\end{tabular}




\begin{tabular}{|c|c|c|c|c|}
\hline TVGLNGSTPHERYMDF & 115 & 130 & 0.83 & 0.36 \\
\hline NKTISDGFGHNHSLCHGDFGNL & 879 & 900 & 0.81 & 0.28 \\
\hline EGMFKQLKPELMPSSA & 678 & 693 & 0.76 & 0.21 \\
\hline GGTIVKDGYGRD & 524 & 535 & 0.74 & 0.04 \\
\hline VEQPYVTESSQNKPTVNGKE & 392 & 411 & 0.67 & 0.21 \\
\hline FASGQKVVYKPRSLS & 212 & 226 & 0.66 & 0.22 \\
\hline MHIDKL & 109 & 114 & 0.63 & 0.05 \\
\hline IWEDDRHAAYL & 616 & 626 & 0.6 & 0.31 \\
\hline KQLKPELMPSSAY & 682 & 694 & 0.59 & 0.23 \\
\hline IWEDDRHAAY & 616 & 625 & 0.57 & 0.07 \\
\hline LKQGHSYL & 813 & 820 & 0.56 & 0.12 \\
\hline NGSTPHERYMDF & 119 & 130 & 0.55 & 0.05 \\
\hline FASGQKVVYKPRSLSIDKQFGEF & 212 & 234 & 0.52 & 0.33 \\
\hline EASTHPDYLQEPTRRNKL & 469 & 486 & 0.51 & 0.19 \\
\hline LIWEDDRHAAYL & 615 & 626 & 0.5 & 0.13 \\
\hline LFTNDL & 317 & 322 & 0.49 & 0.13 \\
\hline FKQLKPELMPSSAY & 681 & 694 & 0.49 & 0.15 \\
\hline YAQYTNNPEPKELARKLAISS & 906 & 926 & 0.48 & 0.26 \\
\hline ASTHPDYLQEPTRRNKL & 470 & 486 & 0.47 & 0.17 \\
\hline ATLTNGDWTPSHEKTPMSPASA & 572 & 593 & 0.46 & 0.08 \\
\hline LARKLAISSIDQAHTYG & 918 & 934 & 0.46 & 0.18 \\
\hline STHPDYLQEPTRRNKL & 471 & 486 & 0.45 & 0.17 \\
\hline ARELTQSVF & 335 & 343 & 0.45 & 0.04 \\
\hline ALELPSSTLTEKELRIHDR & 972 & 990 & 0.42 & 0.22 \\
\hline LFTNDLDC & 317 & 324 & 0.41 & 0.06 \\
\hline LEASTHPDYLQEPTRRNKL & 468 & 486 & 0.41 & 0.08 \\
\hline YEFIPHQEA & 261 & 269 & 0.4 & 0.11 \\
\hline SAVGGGKGVQSE & 363 & 374 & -0.58 & 0.2 \\
\hline
\end{tabular}

[T386A/D387A] - [T386A/D387A: AMPPNP: HalA2]

$\begin{array}{lllll}\text { AVTVSPLTPGIY } & 636 & 647 & 2.55 & 0.16 \\ \text { AVTVSPLTPGIYDGTLG } & 636 & 652 & 2.54 & 0.24 \\ \text { EAVTVSPLTPGIYDGTLG } & 635 & 652 & 2.52 & 0.24 \\ \text { EAVTVSPLTPGIYDGTL } & 635 & 651 & 2.49 & 0.17 \\ \text { ARELTQSVF } & 335 & 343 & 2.43 & 0.12 \\ \text { AVTVSPLTPGIYDGTL } & 636 & 651 & 2.27 & 0.28 \\ \text { EAVTVSPLTPGIY } & 635 & 647 & 2.22 & 0.18 \\ \text { GTLMLPITIASGKL } & 344 & 357 & 1.51 & 0.13 \\ \text { GTLMLPITIASGKLLD } & 344 & 359 & 1.47 & 0.15 \\ \text { ARQIRYIKSSL } & 561 & 571 & 1.46 & 0.18 \\ \text { IRYIKSSL } & 564 & 571 & 1.27 & 0.13 \\ \text { VEQPYVTESSQNKPTVNGKEAN } & 392 & 413 & 0.91 & 0.13\end{array}$




\begin{tabular}{|c|c|c|c|c|}
\hline LFGATDL & 291 & 297 & 0.87 & 0.06 \\
\hline EFIPHQEATSED & 262 & 273 & 0.84 & 0.22 \\
\hline VSVGMNE & 629 & 635 & 0.82 & 0.12 \\
\hline YLFGATDL & 290 & 297 & 0.81 & 0.09 \\
\hline FASGQKVVYKPRSLS & 212 & 226 & 0.77 & 0.26 \\
\hline VSVGMN & 629 & 634 & 0.77 & 0.09 \\
\hline $\begin{array}{l}\text { ATLTNGDWTPSHEKTPMSPASADREDGY } \\
\mathrm{F}\end{array}$ & 572 & 600 & 0.77 & 0.26 \\
\hline LFTNDL & 317 & 322 & 0.76 & 0.12 \\
\hline IQKYFHAENDLF & 40 & 51 & 0.71 & 0.09 \\
\hline QAHTYGWKLGLNHSDQLQG & 929 & 947 & 0.7 & 0.35 \\
\hline IWEDDRHAAYL & 616 & 626 & 0.68 & 0.29 \\
\hline MAPFKKIVPHE & 497 & 507 & 0.66 & 0.21 \\
\hline VEQPYVTESSQNKPTVNGKE & 392 & 411 & 0.63 & 0.36 \\
\hline YEFIPHQEATSE & 261 & 272 & 0.62 & 0.2 \\
\hline APFKKIVPHE & 498 & 507 & 0.62 & 0.12 \\
\hline FGATDLHL & 292 & 299 & 0.61 & 0.42 \\
\hline KQLKPELMPSSA & 682 & 693 & 0.61 & 0.16 \\
\hline EGMFKQLKPELMPSSA & 678 & 693 & 0.56 & 0.1 \\
\hline LKQGHSYL & 813 & 820 & 0.56 & 0.08 \\
\hline PFKKIVPHE & 499 & 507 & 0.54 & 0.13 \\
\hline ASTHPDYLQEPTRRNKL & 470 & 486 & 0.53 & 0.25 \\
\hline KQLKPELMPSSAY & 682 & 694 & 0.5 & 0.16 \\
\hline LIWEDDRHAAYL & 615 & 626 & 0.5 & 0.17 \\
\hline YEFIPHQEA & 261 & 269 & 0.49 & 0.17 \\
\hline LFTNDLDC & 317 & 324 & 0.47 & 0.09 \\
\hline IWEDDRHAAY & 616 & 625 & 0.47 & 0.13 \\
\hline LPITIASGKL & 348 & 357 & 0.46 & 0.14 \\
\hline IERVTHRLQQLGSEDE & 545 & 560 & 0.46 & 0.35 \\
\hline ALELPSSTLTEKELRIHDR & 972 & 990 & 0.43 & 0.27 \\
\hline FKQLKPELMPSSAY & 681 & 694 & 0.42 & 0.2 \\
\hline SAVGGGKGVQSE & 363 & 374 & -0.79 & 0.46 \\
\hline \multicolumn{5}{|c|}{ [GS389-396] - [GS389-396: AMPPNP: HalA2] } \\
\hline AVTVSPLTPGIY & 636 & 647 & 1.68 & 0.14 \\
\hline EAVTVSPLTPGIY & 635 & 647 & 1.41 & 0.24 \\
\hline AVTVSPLTPGIYDGTLG & 636 & 652 & 1.11 & 0.27 \\
\hline ARQIRYIKSSL & 561 & 571 & 1.09 & 0.11 \\
\hline ARELTQSVF & 335 & 343 & 1.06 & 0.06 \\
\hline IRYIKSSL & 564 & 571 & 1.05 & 0.08 \\
\hline EAVTVSPLTPGIYDGTLG & 635 & 652 & 0.95 & 0.35 \\
\hline AVTVSPLTPGIYDGTL & 636 & 651 & 0.72 & 0.42 \\
\hline
\end{tabular}




\begin{tabular}{|c|c|c|c|c|}
\hline IWEDDRHAAYL & 616 & 626 & 0.68 & 0.36 \\
\hline $\begin{array}{l}\text { ATLTNGDWTPSHEKTPMSPASADREDGY } \\
\mathrm{F}\end{array}$ & 572 & 600 & 0.61 & 0.21 \\
\hline IQKYFHAENDLF & 40 & 51 & 0.61 & 0.12 \\
\hline LYAQYTNNPEPKE & 905 & 917 & 0.59 & 0.22 \\
\hline FASGQKVVYKPRSLS & 212 & 226 & 0.56 & 0.17 \\
\hline GTLMLPITIASGKL & 344 & 357 & 0.55 & 0.1 \\
\hline EGMFKQLKPELMPSSA & 678 & 693 & 0.53 & 0.08 \\
\hline LFTNDL & 317 & 322 & 0.53 & 0.05 \\
\hline LKQGHSYL & 813 & 820 & 0.48 & 0.14 \\
\hline EFIPHQEATSED & 262 & 273 & 0.47 & 0.09 \\
\hline IWEDDRHAAY & 616 & 625 & 0.47 & 0.07 \\
\hline LFGATDL & 291 & 297 & 0.46 & 0.08 \\
\hline LFTNDLDC & 317 & 324 & 0.44 & 0.06 \\
\hline MDHNGPIFA & 440 & 448 & 0.43 & 0.09 \\
\hline LIWEDDRHAAYL & 615 & 626 & 0.43 & 0.17 \\
\hline ASTHPDYLQEPTRRNKL & 470 & 486 & 0.42 & 0.11 \\
\hline MAPFKKIVPHE & 497 & 507 & 0.42 & 0.19 \\
\hline ALELPSSTLTEKELRIHDR & 972 & 990 & 0.42 & 0.29 \\
\hline KQLKPELMPSSA & 682 & 693 & 0.41 & 0.06 \\
\hline WVIVNQ & 379 & 384 & -0.54 & 0.32 \\
\hline SAVGGGKGVQSE & 363 & 374 & -0.91 & 0.36 \\
\hline \multicolumn{5}{|c|}{ [GS341-348] - [GS341-348: AMPPNP: HalA2] } \\
\hline HIDKLTVGL & 110 & 118 & 1.17 & 0.27 \\
\hline TVGLNGSTPHERYMDF & 115 & 130 & 0.64 & 0.31 \\
\hline FASGQKVVYKPRSLS & 212 & 226 & 0.62 & 0.16 \\
\hline PITIASGKL & 349 & 357 & 0.62 & 0.15 \\
\hline MHIDKL & 109 & 114 & 0.57 & 0.07 \\
\hline IWEDDRHAAYL & 616 & 626 & 0.54 & 0.33 \\
\hline LKQGHSYL & 813 & 820 & 0.54 & 0.09 \\
\hline YSRIGGY & 279 & 285 & 0.45 & 0.09 \\
\hline AVTVSPLTPGIYDGTL & 636 & 651 & -0.44 & 0.21 \\
\hline ARQIRYIKSSL & 561 & 571 & -0.51 & 0.15 \\
\hline SSQNKPTVNGKEANIGNYIPHVTDG & 400 & 424 & -0.56 & 0.31 \\
\hline QAHTYGWKLGLNHSDQLQG & 929 & 947 & -0.71 & 0.26 \\
\hline EAVTVSPLTPGIYDGTL & 635 & 651 & -0.74 & 0.19 \\
\hline SAVGGGKGVQSE & 363 & 374 & -1.17 & 0.26 \\
\hline \multicolumn{5}{|c|}{ [P349A] - [P349A: AMPPNP: HalA2] } \\
\hline HIDKLTVGL & 110 & 118 & 1.01 & 0.25 \\
\hline AVTVSPLTPGIY & 636 & 647 & 0.88 & 0.09 \\
\hline IRYIKSSL & 564 & 571 & 0.86 & 0.16 \\
\hline
\end{tabular}




\begin{tabular}{|c|c|c|c|c|}
\hline ARQIRYIKSSL & 561 & 571 & 0.86 & 0.05 \\
\hline EAVTVSPLTPGIY & 635 & 647 & 0.68 & 0.17 \\
\hline IWEDDRHAAYL & 616 & 626 & 0.55 & 0.33 \\
\hline MHIDKL & 109 & 114 & 0.54 & 0.07 \\
\hline FASGQKVVYKPRSLS & 212 & 226 & 0.53 & 0.15 \\
\hline TVGLNGSTPHERYMDF & 115 & 130 & 0.52 & 0.31 \\
\hline LKQGHSYL & 813 & 820 & 0.52 & 0.07 \\
\hline AVTVSPLTPGIYDGTLG & 636 & 652 & 0.44 & 0.11 \\
\hline ARELTQSVF & 335 & 343 & 0.42 & 0.07 \\
\hline SSQNKPTVNGKEANIGNYIPHVTDG & 400 & 424 & -0.44 & 0.23 \\
\hline QAHTYGWKLGLNHSDQLQG & 929 & 947 & -0.52 & 0.2 \\
\hline IEWVNSKGFQPSLRI & 235 & 249 & -0.53 & 0.09 \\
\hline VEQPYVTESSQNKPTVNGKEAN & 392 & 413 & -0.78 & 0.16 \\
\hline SAVGGGKGVQSE & 363 & 374 & -0.94 & 0.31 \\
\hline \multicolumn{5}{|c|}{ [P405A] - [P405A: AMPPNP: HalA2] } \\
\hline ARELTQSVF & 335 & 343 & 2.16 & 0.06 \\
\hline GTLMLPITIASGKL & 344 & 357 & 1.3 & 0.12 \\
\hline AVTVSPLTPGIY & 636 & 647 & 1.26 & 0.04 \\
\hline FKEKDLRL & 183 & 190 & 1.23 & 0.25 \\
\hline AVTVSPLTPGIYDGTLG & 636 & 652 & 1.2 & 0.08 \\
\hline HIDKLTVGL & 110 & 118 & 1.2 & 0.29 \\
\hline EAVTVSPLTPGIYDGTLG & 635 & 652 & 1.17 & 0.1 \\
\hline GTLMLPITIASGKLLD & 344 & 359 & 1.14 & 0.08 \\
\hline EAVTVSPLTPGIY & 635 & 647 & 1.14 & 0.22 \\
\hline AVTVSPLTPGIYDGTL & 636 & 651 & 1.11 & 0.09 \\
\hline EAVTVSPLTPGIYDGTL & 635 & 651 & 1.06 & 0.11 \\
\hline IRYIKSSL & 564 & 571 & 1.03 & 0.05 \\
\hline $\begin{array}{l}\text { ATLTNGDWTPSHEKTPMSPASADREDGY } \\
\mathrm{F}\end{array}$ & 572 & 600 & 0.74 & 0.14 \\
\hline ARQIRYIKSSL & 561 & 571 & 0.72 & 0.09 \\
\hline EFIPHQEATSED & 262 & 273 & 0.7 & 0.09 \\
\hline ASTHPDYLQEPTRRNKL & 470 & 486 & 0.69 & 0.04 \\
\hline YEFIPHQEATSE & 261 & 272 & 0.67 & 0.05 \\
\hline YLFGATDL & 290 & 297 & 0.67 & 0.04 \\
\hline TVGLNGSTPHERYMDF & 115 & 130 & 0.65 & 0.27 \\
\hline VSVGMNE & 629 & 635 & 0.64 & 0.05 \\
\hline EASTHPDYLQEPTRRNKL & 469 & 486 & 0.6 & 0.11 \\
\hline YEFIPHQEA & 261 & 269 & 0.59 & 0.24 \\
\hline LFGATDL & 291 & 297 & 0.59 & 0.03 \\
\hline LEASTHPDYLQEPTRRNKL & 468 & 486 & 0.58 & 0.07 \\
\hline EVAKTTASRL & 764 & 773 & 0.58 & 0.07 \\
\hline
\end{tabular}




$\begin{array}{lrrrr}\text { FASGQKVVYKPRSLS } & 212 & 226 & 0.58 & 0.2 \\ \text { VAKTTASRL } & 765 & 773 & 0.56 & 0.07 \\ \text { VSVGMN } & 629 & 634 & 0.56 & 0.04 \\ \text { STHPDYLQEPTRRNKL } & 471 & 486 & 0.56 & 0.25 \\ \text { MHIDKL } & 109 & 114 & 0.55 & 0.04 \\ \text { FGATDLHL } & 292 & 299 & 0.55 & 0.42 \\ \text { KQLKPELMPSSAY } & 682 & 694 & 0.54 & 0.2 \\ \text { MLPITIASGKL } & 347 & 357 & 0.54 & 0.09 \\ \text { ALELPSSTLTEKELRIHDR } & 972 & 990 & 0.52 & 0.17 \\ \text { IWEDDRHAAYL } & 616 & 626 & 0.52 & 0.39 \\ \text { LYAQYTNNPEPKE } & 905 & 917 & 0.52 & 0.32 \\ \text { FKQLKPELMPSSAY } & 681 & 694 & 0.51 & 0.16 \\ \text { YSRIGGY } & 279 & 285 & 0.5 & 0.04 \\ \text { ATLTNGDWTPSHEKTPMSPASA } & 572 & 593 & 0.46 & 0.18 \\ \text { EGMFKQLKPELMPSSA } & 678 & 693 & 0.45 & 0.1 \\ \text { ACGEHPML } & 304 & 311 & 0.45 & 0.04 \\ \text { MDHNGPIFA } & 440 & 448 & 0.45 & 0.07 \\ \text { MAPFKKIVPHE } & 497 & 507 & 0.44 & 0.22 \\ \text { LKQGHSYL } & 813 & 820 & 0.44 & 0.09 \\ \text { GVGDTHVNGQC } & 196 & 206 & 0.44 & 0.25 \\ \text { KQLKPELMPSSA } & 682 & 693 & 0.43 & 0.07 \\ \text { FKQLKPELMPSSA } & 681 & 693 & 0.42 & 0.32 \\ \text { LQEPTRRNKL } & 477 & 486 & 0.42 & 0.13 \\ \text { EFIPHQEA } & 262 & 269 & 0.41 & 0.08 \\ \text { NHSDQLQG } & 940 & 947 & 0.41 & 0.08\end{array}$


[1] Habibi, Y., Uggowitzer, K. A., Issak, H., and Thibodeaux, C. J. (2019) Insights into the Dynamic Structural Properties of a Lanthipeptide Synthetase using Hydrogen-Deuterium Exchange Mass Spectrometry, J. Am. Chem. Soc. 141, 14661-14672.

[2] Thibodeaux, C. J., Ha, T., and van der Donk, W. A. (2014) A Price to Pay for Relaxed Substrate Specificity: A Comparative Kinetic Analysis of the Class II Lanthipeptide Synthetases, ProcM and HalM2, J. Am. Chem. Soc. 136, 17513-17529.

[3] Johnson, K. A., Simpson, Z. B., and Blom, T. (2009) Global Kinetic Explorer: A new computer program for dynamic simulation and fitting of kinetic data, Anal. Biochem. 387, 20-29.

[4] Johnson, K. A., Simpson, Z. B., and Blom, T. (2009) FitSpace Explorer: An algorithm to evaluate multi-dimensional parameter space in fitting kinetic data, Anal. Biochem. 387, 30-41.

[5] Thibodeaux, G. N., McClerren, A. L., Ma, Y., Gancayco, M. R., and van der Donk, W. A. (2015) Synergistic binding of the leader and core peptides by the lantibiotic synthetase HalM2, ACS Chem. Biol. 10, 970-977.

[6] Krenske, E. H., Petter, R. C., Zhu, Z., and Houk, K. N. (2011) Transition states and energetics of nucleophilic additions of thiols to substituted alpha,beta-unsaturated ketones: substituent effects involve enone stabilization, product branching, and solvation, J. Org. Chem. 76, 5074-5081.

[7] Yang, X., and van der Donk, W. A. (2015) Michael-Type Cyclizations in Lantibiotic Biosynthesis Are Reversible, ACS Chem. Biol. 10, 1234-1238.

[8] Zhang, Q., Ortega, M. A., Shi, Y., Wang, H., Melby, J. O., Mitchell, D. A., and van der Donk, W. A. (2014) Structural and biosynthetic investigation of RiPPs by hypothetical structure enumeration and evaluation using tandem mass spectrometry, Proc. Natl Acad. Sci. U.S.A. 111, 12031-12036.

[9] Habibi, Y., Uggowitzer, K. A., Issak, H., and Thibodeaux, C. J. (2019) Insights into the Dynamic Structural Properties of a Lanthipeptide Synthetase using Hydrogen-Deuterium Exchange Mass Spectrometry, Journal of the American Chemical Society 141, 14661-14672.

[10] Waterhouse, A., Bertoni, M., Bienert, S., Studer, G., Tauriello, G., Gumienny, R., Heer, F. T., de Beer, T. A. P., Rempfer, C., Bordoli, L., Lepore, R., and Schwede, T. (2018) SWISSMODEL: homology modelling of protein structures and complexes, Nucleic. Acids. Res. 46, W296-W303.

[11] Jorgensen, W. L., Chandrasekhar, J., Madura, J. D., Impey, R. W., and Klein, M. L. (1983) Comparison of Simple Potential Functions for Simulating Liquid Water, J. Chem. Phys. 79, 926-935.

[12] D.A. Case, K. B., I.Y. Ben-Shalom, S.R. Brozell, D.S. Cerutti, T.E. Cheatham, III, V.W.D. Cruzeiro, T.A. Darden, R.E. Duke, G. Giambasu, M.K. Gilson, H. Gohlke, A.W. Goetz,R Harris, S. Izadi, K. Kasava- jhala, A. Kovalenko, R. Krasny, T. Kurtzman, T.S. Lee, S. LeGrand, P. Li, C. Lin, J. Liu, T. Luchko, R. Luo, V. Man, K.M. Merz, Y. Miao, O. Mikhailovskii, G. Monard, H. Nguyen, A. Onufriev, F. Pan, S. Pantano, R. Qi, D.R. Roe, A. Roitberg, C. Sagui, S. Schott-Verdugo, J. Shen, C.L. Simmerling, N. Skrynnikov, J. Smith, J. Swails, R.C. Walker, J. Wang, L. Wilson, R.M. Wolf, X. Wu, D.M. York and P.A. Kollman. (2020) AMBER 2020, University of California, San Francisco.

[13] Salomon-Ferrer, R., Götz, A. W., Poole, D., Le Grand, S., and Walker, R. C. (2013) Routine Microsecond Molecular Dynamics Simulations with AMBER on GPUs. 2. Explicit Solvent Particle Mesh Ewald, J. Chem. Theory Comput. 9, 3878-3888.

[14] Maier, J. A., Martinez, C., Kasavajhala, K., Wickstrom, L., Hauser, K. E., and Simmerling, C. (2015) ff14SB: Improving the Accuracy of Protein Side Chain and Backbone Parameters from ff99SB, J Chem Theory Comput 11, 3696-3713.

[15] Andersen, H. C. (1980) Molecular dynamics simulations at constant pressure and/or temperature, The Journal of Chemical Physics 72, 2384-2393. 
[16] Elber, R., Ruymgaart, A. P., and Hess, B. (2011) SHAKE parallelization, Eur Phys J Spec Top 200, 211-223.

[17] Feig, M., Karanicolas, J., and Brooks, C. L., 3rd. (2004) MMTSB Tool Set: enhanced sampling and multiscale modeling methods for applications in structural biology, J. Mol. Graph. Model. 22, 377-395. 